\title{
INTRACRANIAL VESSEL WALL MR IMAGING From Bench to Bedside
}

Arjen Lindenholz 
Intracranial Vessel Wall MR Imaging: From Bench to Bedside PhD Thesis, Utrecht University, The Netherlands

Cover design en lay-out: Publiss | www.publiss.nl

Print:

Ridderprint | www.ridderprint.nl

ISBN:

978-94-6416-508-1

DOI: $\quad$ https://doi.org/10.33540/507

All rights reserved. No part of this publication may be reproduced in any form or by any means, without permission in writing of the author. The copyright of the articles that have been accepted for publication or that have already been published, has been transferred to the respective journals.

This research is supported by the European Research Council (grant agreement number 637024)

Financial support by the Dutch Heart Foundation for the publication of this thesis was gratefully acknowledged

Financial support for the publication of this thesis was kindly provided by: Angiocare BV, Bayer B.V. Pharmaceuticals - Radiology, Terumo Europe NV, Guerbet Nederland BV, ChipSoft BV, Sectra Benelux, Pie Medical Imaging BV and Penumbra Europe $\mathrm{GmbH}$.

(c) 2021 Arjen Lindenholz 


\title{
Intracranial Vessel Wall MR Imaging: From Bench to Bedside
}

\author{
Intracraniële Vaatwand MRI: \\ Van onderzoek naar kliniek \\ (met een samenvatting in het Nederlands)
}

\section{Proefschrift}

ter verkrijging van de graad van doctor aan

de Universiteit Utrecht op gezag van de rector magnificus, prof. dr. H.R.B.M. Kummeling, ingevolge het besluit van het college voor promoties in het openbaar te verdedigen op

donderdag 10 juni 2021 des middags te 4:15 uur

door

\section{Arjen Lindenholz}

geboren op 18 december 1986

te Staphorst 
Promotor:

Copromotoren:
Prof. dr. J. Hendrikse

Prof. dr. H.B. van der Worp

Dr. I.C. van der Schaaf

Dr. A.G. van der Kolk 
Voor mijn ouders,

Die mij altijd hebben gestimuleerd om het maximale uit mijzelf te halen en niet op te geven. 


\title{
Table of Contents
}

\author{
Introduction
}

Chapter $1 \quad$ General Introduction

Part I

Technical Aspects, Challenges and Assessment

Chapter $2 \quad$ Clinical Vascular Imaging in the Brain at 7T

Chapter 3 The Use and Pitfalls of Intracranial Vessel Wall Imaging: How We Do It

Chapter $4 \quad$ Comparison of 3T Intracranial Vessel Wall MRI

Part II

Chapter 5

Chapter 6

Chapter 7

\section{Sequences}

\section{Clinical Trans/ation}

Intracranial Atherosclerosis Assessed with 7T MRI: Evaluation of Patients with Ischemic Stroke or Transient Ischemic Attack

Cerebral Parenchymal Changes at 7T MRI in Patients with TIA or Ischemic Stroke

MRI Vessel Wall Imaging after Intra-Arterial Treatment for Acute Ischemic Stroke

Summary \& General Discussion

Chapter 8 Summary

Chapter 9 General Discussion

\section{Appendices}

Addendum I Dutch Summary (Nederlandse samenvatting)

Addendum II List of Publications

Addendum III Acknowledgements (Dankwoord)

Addendum IV Curriculum vitae 


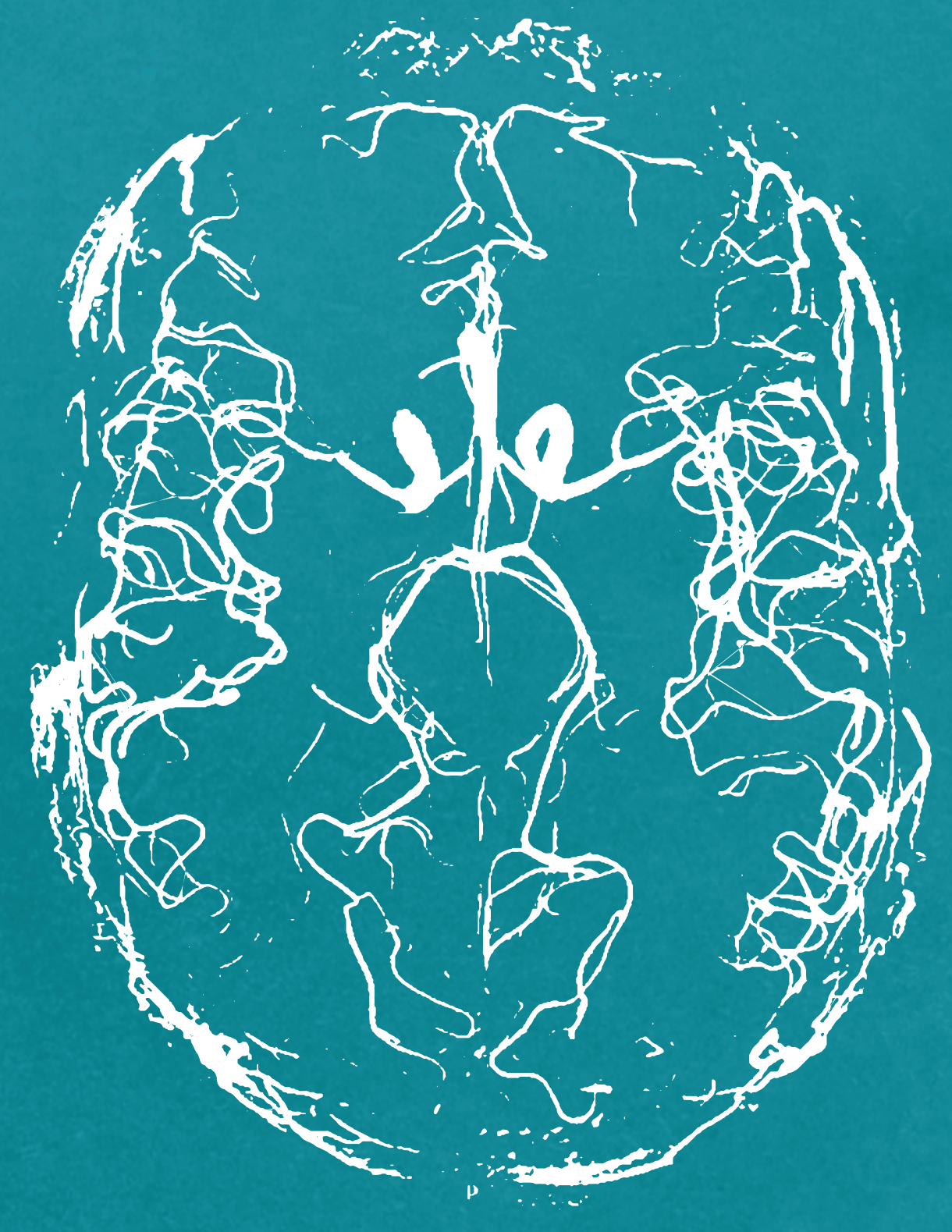


CHAPTER

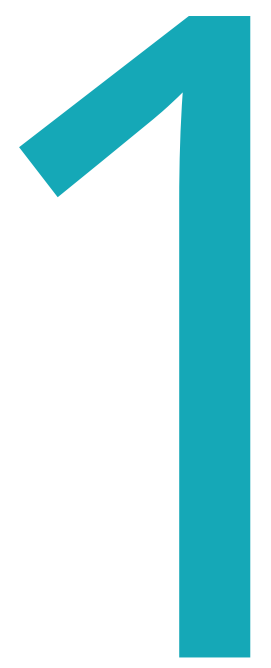

\section{General Introduction}


Intracranial vessel wall magnetic resonance imaging (MRI) is an emerging technique that has gained an increasing popularity in the last decade because of its potential applications in a variety of intracranial vascular diseases. ${ }^{1-4}$ This thesis will address both technical aspects and developments of intracranial vessel wall MRI as well as indications and implications of this technique for clinical practice.

\section{A history of vessel wall MR imaging}

At the time when vessel wall MR imaging of the intracranial arteries was first introduced in 1995 using a 1.5 tesla (T) MRI platform, the technique had been limited to the larger extracranial arteries such as the vertebral and cervical carotid arteries..$^{5}$ These arteries could be readily assessed with vessel wall MRI due to their relatively large size, and they were of specific interest because of being a predilection site for atherosclerotic plaques, especially the carotids. ${ }^{6,7}$ With vessel wall MRI important information beyond the lumen of the extracranial arteries could be perceived: not only the exact location and severity of the (stenotic) atherosclerotic plaque, but also the extent of the non-stenotic atheromatous part of the plaque that had not (yet) resulted in luminal narrowing due to outward arterial remodeling of the affected artery. ${ }^{8,9}$ It had already been recognized through pathology and lumenographic studies that the presence of (unstable) atherosclerotic plaques could cause hemodynamically significant stenosis and thromboembolism, potentially leading to ischemic stroke or transient ischemic attack (TIA). The reliability of this MR imaging technique was further validated by histopathological examination of the material obtained through carotid endarterectomy procedures. ${ }^{10,11}$

Over the subsequent decade, higher magnetic field strength MRI platforms like 3T - due to their higher attainable signal-to-noise ratio (SNR) and spatial resolution - facilitated more detailed atherosclerotic plaque imaging and the recognition of the 'vulnerable' plaque as atherosclerotic culprit lesion, further supporting the importance of vessel wall MR imaging in the diagnostic workup of cerebral ischemic events. Simultaneously, interest in visualizing the vessel walls of the intracranial arteries was growing as increasing evidence of predominantly lumenographic studies showed that intracranial atherosclerosis (ICAS) and stenosis also play an important causative role in ischemic stroke and TIA, especially in the Asian population. ${ }^{12}$ The intracranial arterial vessel wall, however, was still a relatively unexplored area because visualizing these vessel walls posed several challenges given the deep location, the smaller size and tortuosity of the intracranial arteries. With the advent of $3 T$ platforms and the development of higher-resolution extracranial vessel wall MRI sequences, new opportunities arose to visualize the sub-millimeter thin intracranial vessel wall as well. ${ }^{13-15}$ 


\section{Challenges in visualizing the intracranial vessel wall}

Compared with extracranial vessel wall sequences, several unique challenges can be identified for intracranial vessel wall MRI. Chapter 3 provides an overview of these challenges in technical requirements and assessment of these images. First, most early MRI sequences were 'copy-pasted' from extracranial vessel wall MRI as two-dimensional (2D) acquisitions with anisotropic voxel size. ${ }^{14,16,17}$ Although this does not pose significant issues in the larger extracranial arteries due to their mostly straight configuration, it caused hindering partial volume artefacts in the smaller, more tortuous intracranial arteries. Another drawback of 2D acquired vessel wall MR images is that the intracranial vessel wall and atherosclerotic plaque cannot be assessed in multiple planes without necessitating acquisitions in all three directions (axial, sagittal and coronal), tripling the total acquisition time. 3D intracranial vessel wall MRI sequences are therefore gaining popularity, allowing for isotropic acquisition which renders multiplanar reformatting of the vessel wall images possible. However, (in-plane) spatial resolution of these sequences is generally lower than of the $2 \mathrm{D}$ sequences. ${ }^{15}$

Second, a high contrast-to-noise ratio (CNR) is required to delineate the thin intracranial vessel wall from the surrounding intraluminal blood and extraluminal CSF. Black blood techniques are used in extracranial and intracranial vessel wall MRI to suppress the signal from the intraluminal blood. Whereas the extracranial arteries are surrounded by fatty and other tissues, the intracranial arteries are also surrounded by variably flowing cerebrospinal fluid (CSF). This means that, in addition to black blood techniques, the signal from the surrounding CSF also needs to be suppressed. Initial intracranial vessel wall MRI sequences had difficulty discerning the vessel wall from both the arterial lumen (because of the different flow directions of the tortuous intracranial arteries) and from the surrounding CSF. ${ }^{5}$ A variety of (flow saturation) techniques have been developed to suppress the MR signal from blood and CSF, mostly relying on the flow properties of luminal blood (black blood sequences) and the use of specific preparation pulses (double inversion recovery pulses), albeit with varying success in improving the delineation of the intracranial vessel wall. 2,15,16,18,19

Third, although the technical developments have made clear visualization of the small and tortuous intracranial vessel wall possible with improved spatial resolution, SNR and CNR, an important drawback of all these highly-demanding requirements are the relative long acquisition times. Long scan times induce motion artifacts, for example when examining stroke and TIA patients who are often elderly with moderate to severe neurological disability and therefore prone to movement. This also limits the extent of atherosclerotic plaque characterization that is possible. For instance, a choice often has to be made between acquiring a $\mathrm{T}_{1}$-weighted vessel wall sequence before and after contrast administration, 
which will most reliably show enhancement, or suffice with a postcontrast $T_{1}$ weighted sequence alone. Improvements in this area are ongoing, and various innovative techniques are being investigated to achieve a reduction in acquisition time while maintaining acceptable image quality (Chapter 4).20,21

\section{T versus 7T MRI}

As discussed, intracranial vessel wall MRI requires a high SNR - translating to a high spatial resolution and high CNR - to obtain clear visualization of the vessel wall. As the SNR increases with increasing magnetic field strength, the step towards > 3T MRI platforms for intracranial vessel wall MR imaging seems inevitable. Although 7T MRI platforms have been research-only until October 2017, over the last two decades many new techniques and applications have been generated and tested at $7 \mathrm{~T}$ worldwide, for a wide variety of clinical and radiological purposes (Chapter 2). One main field of interest of the research department of the University Medical Center Utrecht has been neuroimaging, and in 2009 the first intracranial 7T vessel wall MRI sequence was developed, followed by several technical improvements in hardware and field-of-view (FOV) in the subsequent years. Initial results showed high quality of visualization of the intracranial vessel wall and clear assessment of vessel wall abnormalities. ${ }^{16,22-24}$ Three subsequent large clinical studies using $7 \mathrm{~T}$ intracranial vessel wall MR imaging have recently been completed; the Intracranial Vessel wall Imaging (IVI) study, the Posterior Intracranial Vessel wall Imaging (PIVI) study and Diagnosing Cerebral Vasculitis (DIVA) study. They provide detailed assessment of respectively the intracranial vessel wall in healthy adults, patients with anterior or posterior ischemic stroke and TIA, and patients with central nervous system vasculitis. Data of the IVI study has been used in this thesis for clinical correlation (Chapters 5-7). In general, 7T MR imaging has been shown to be superior to 3T MRI in visualizing the intracranial vessel wall, enabling assessment of even very small vessel wall abnormalities that are not visible with lower field strengths. ${ }^{25,26}$ An additional advantage of 7T is the relative ease with which CSF suppression is achieved, something that has not been matched at 3T yet. Finally, high SNRs and CNRs of 7T MRI enable more detailed assessment of other neurological pathology like (micro)infarcts and (micro)bleeds, which together with highly detailed vessel wall MR imaging might provide information on the cerebrovascular status of the individual patient. ${ }^{27,28}$ However, the question remains whether these advantages have additional clinical value over data acquired at $3 \mathrm{~T}$.

Nevertheless, for all its advantages, a major drawback of 7T MRI is that these platforms are only sparsely available, which makes widespread implementation of 7T MRI sequences in clinical practice currently impossible. Therefore, it is important that technical advancements and newly acquired insights can be translated back to 3T MR systems (or even lower MR field strength platforms). 


\section{Clinical consequence of intracranial vessel wall lesions}

For both 3T and 7T MRI platforms, a number of intracranial vessel wall MR imaging sequences are currently available that provide clear visibility of the vessel wall and its pathology. ${ }^{13,15,19,21,22,26,29}$ In addition, several (pictorial) reviews have recently been published addressing the current and potential clinical capabilities of intracranial vessel wall imaging in a variety of vascular pathologies..$^{1-4}$ As a result, an increasing number of radiologists and non-radiology clinicians are interested in implementing intracranial vessel wall MR imaging in clinical practice. However, apart from aiding diagnosis of central nervous system vasculitis by assessing specific vessel wall enhancement patterns, clear advantages for clinical decisionmaking are currently lacking. ${ }^{20}$ One important reason is that - contrary to the extracranial arteries - histopathological validation (in vivo versus ex vivo) of intracranial vessel wall MR findings is virtually impossible to perform due to the lack of pathological specimens from living patients. An alternative option, solely ex vivo validation studies, show promising results, but are limited. ${ }^{30-32}$ Therefore, the true nature of vessel wall MRI findings and their prevalence among different ethnicities has not been completely determined yet.

Several alternative, more indirect methods of deducing the nature of intracranial vessel wall MR lesions are available. By studying their association with other established cerebrovascular markers and imaging findings, an assumption can be made as to the exact nature of these (at least most) lesions. For instance, if the presence and number of lesions coincide with the presence (and extent) of cerebrovascular risk factors like smoking and hypertension, they will more likely be atherosclerotic plaques (Chapter 5). Furthermore, this method may aid in determining to translate these MR findings in a clinical perspective. This same reasoning may apply for parenchymal damage: if the vessel wall lesions that we see are true atherosclerotic plaques and thereby indicative of a certain atherosclerotic burden, we would expect more ischemic damage in patients with a high lesion load (Chapter 6). This would also provide insight into the clinical value of visualizing these intracranial vessel wall lesions on MRI. Alternatively, an assumption can be made as to the exact nature of these lesions by monitoring the behavior of vessel wall lesions over time. Pre- and posttreatment intracranial vessel wall MRI or follow-up examinations may provide additional information in the development of a vessel wall lesion or vessel wall status. For example, lesions that disappear during follow-up without treatment are more likely transient in nature or iatrogenic in case of previous treatment with mechanical thrombectomy (Chapter 7). 


\section{This Thesis}

This thesis will address several of the current caveats in both implementation of intracranial vessel wall MRI sequences and clinical interpretation of imaging findings as described above, thereby providing several necessary steps in the transition from research-based knowledge towards a radiological and clinically applicable imaging technique, both at 3T and 7T MRI. 


\section{References}

1. Bhogal P, Navaei E, Makalanda HL, et al. Intracranial vessel wall MRI. Clinical Radiology 2016;71:293-303.

2. Mandell DM, Mossa-Basha M, Qiao Y, et al. Intracranial Vessel Wall MRI: Principles and Expert Consensus Recommendations of the American Society of Neuroradiology. AJNR American Journal of Neuroradiology 2017;38:218-229.

3. Dieleman N, van der Kolk AG, Zwanenburg Jl, et al. Imaging intracranial vessel wall pathology with magnetic resonance imaging: current prospects and future directions. Circulation 2014;130:192-201.

4. Mossa-Basha M, Alexander M, Gaddikeri S, Yuan C, Gandhi D. Vessel wall imaging for intracranial vascular disease evaluation. Journal of Neurointerventional Surgery 2016;8:1154-1159.

5. Aoki S, Shirouzu I, Sasaki Y, et al. Enhancement of the intracranial arterial wall at MR imaging: relationship to cerebral atherosclerosis. Radiology 1995;194:477-81.

6. Yuan C, Mitsumori LM, Beach KW, Maravilla KR. Carotid atherosclerotic plaque: noninvasive MR characterization and identification of vulnerable lesions. Radiology 2001;221:285-99.

7. Wasserman BA, Wityk RJ, Trout HH, 3rd, Virmani R. Low-grade carotid stenosis: looking beyond the lumen with MRI. Stroke 2005;36:2504-13.

8. Gutierrez J, Goldman J, Honig LS, Elkind MS, Morgello S, Marshall RS. Determinants of cerebrovascular remodeling: do large brain arteries accommodate stenosis? Atherosclerosis 2014;235:371-9.

9. Qiao Y, Anwar Z, Intrapiromkul J, et al. Patterns and Implications of Intracranial Arterial Remodeling in Stroke Patients. Stroke 2016;47:434-40.

10. Lopez Gonzalez MR, Foo SY, Holmes WM, et al. Atherosclerotic Carotid Plaque Composition: A 3T and 7T MRI-Histology Correlation Study. Journal of Neuroimaging 2016;26:406-13.

11. Pan XM, Saloner D, Reilly LM, et al. Assessment of carotid artery stenosis by ultrasonography, conventional angiography, and magnetic resonance angiography: correlation with ex vivo measurement of plaque stenosis. Journal of Vascular Surgery $1995 ; 21: 82-8$.

12. Gorelick PB, Wong KS, Bae HJ, Pandey DK. Large artery intracranial occlusive disease: a large worldwide burden but a relatively neglected frontier. Stroke 2008;39:2396-9.

13. Qiao Y, Guallar E, Suri FK, et al. MR Imaging Measures of Intracranial Atherosclerosis in a Population-based Study. Radiology 2016;280:860-8.

14. Swartz RH, Bhuta SS, Farb RI, et al. Intracranial arterial wall imaging using high-resolution 3-tesla contrast-enhanced MRI. Neurology 2009;72:627-34. 
15. Qiao Y, Steinman DA, Qin Q, Etesami M, Schar M, Astor BC. Intracranial arterial wall imaging using three-dimensional high isotropic resolution black blood MRI at 3.0 Tesla. Journal of Magnetic Resonance Imaging : JMRI 2011;34:22-30.

16. Van der Kolk AG, Zwanenburg JJ, Brundel M, et al. Intracranial vessel wall imaging at 7.0T MRI. Stroke 2011;42:2478-84.

17. Chung GH, Kwak HS, Hwang SB, Jin GY. High resolution MR imaging in patients with symptomatic middle cerebral artery stenosis. European Journal of Radiology 2012;81:4069-74.

18. Xie Y, Yang Q, Xie G, Pang J, Fan Z, Li D. Improved black-blood imaging using DANTESPACE for simultaneous carotid and intracranial vessel wall evaluation. Magnetic Resonance in Medicine 2016;75:2286-94.

19. Park JK, Kim SH, Kim BS, Choi G, Jeong SY, Choi JC. Imaging of intracranial plaques with black-blood double inversion recovery MR imaging and CT. Journal of Neuroimaging 2011;21:e64-8.

20. Lindenholz A, van der Kolk AG, Zwanenburg JJM, Hendrikse J. The Use and Pitfalls of Intracranial Vessel Wall Imaging: How We Do It. Radiology 2018;286:12-28.

21. Song JW, Moon BF, Burke MP, et al. MR Intracranial Vessel Wall Imaging: A Systematic Review. Journal of Neuroimaging :2020;30:428-442.

22. Van der Kolk AG, Hendrikse J, Brundel M, Biessels GJ, Smit EJ, Visser F. Multi-sequence whole-brain intracranial vessel wall imaging at 7.0 tesla. European Radiology 2013;23:2996-3004.

23. Van der Kolk AG, Zwanenburg J, Brundel M, et al. Distribution and natural course of intracranial vessel wall lesions in patients with ischemic stroke or TIA at 7.0 Tesla MRI. European Radiology 2015;25:1692-700.

24. Harteveld AA, van der Kolk AG, van der Worp HB, et al. Detecting Intracranial Vessel Wall Lesions With 7T-Magnetic Resonance Imaging: Patients With Posterior Circulation Ischemia Versus Healthy Controls. Stroke 2017;48:2601-4.

25. Harteveld AA, van der Kolk AG, van der Worp HB, et al. High-resolution intracranial vessel wall MRI in an elderly asymptomatic population: comparison of 3T and 7T. European Radiology 2017;27:1585-1595.

26. Zhu $C$, Haraldsson $H$, Tian $B$, et al. High resolution imaging of the intracranial vessel wall at 3 and 7 T using 3D fast spin echo MRI. MAGMA 2016;29:559-70.

27. De Cocker LJ, Lindenholz A, Zwanenburg JJ, et al. Clinical vascular imaging in the brain at 7T. Neurolmage 2018;168:452-8.

28. Van Veluw SJ, Zwanenburg JJ, Hendrikse J, van der Kolk AG, Luijten PR, Biessels GJ. High resolution imaging of cerebral small vessel disease with 7 T MRI. Acta Neurochir Suppl 2014;119:125-30.

29. Lee HN, Ryu CW, Yun SJ. Vessel-Wall Magnetic Resonance Imaging of Intracranial Atherosclerotic Plaque and Ischemic Stroke: A Systematic Review and Meta-Analysis. Frontiers in Neurology 2018;9:1032. 
30. Harteveld AA, Denswil NP, Siero JC, et al. Quantitative Intracranial Atherosclerotic Plaque Characterization at 7T MRI: An Ex Vivo Study with Histologic Validation. AJNR American Journal of Neuroradiology 2016;37:802-10.

31. Yang WJ, Chen XY, Zhao HL, et al. In Vitro Assessment of Histology Verified Intracranial Atherosclerotic Disease by 1.5T Magnetic Resonance Imaging: Concentric or Eccentric? Stroke 2016;47:527-30.

32. Van der KolkAG, ZwanenburgJJ, Denswil NP, et al. Imaging the intracranial atherosclerotic vessel wall using 7T MRI: initial comparison with histopathology. AJNR American Journal of Neuroradiology 2015;36:694-701. 

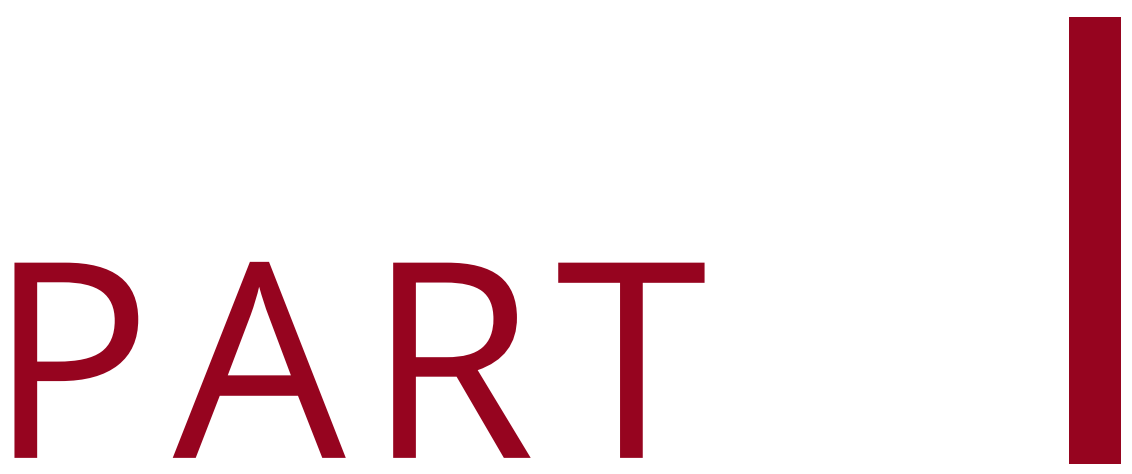

Technical Aspects, Challenges and Assessment 


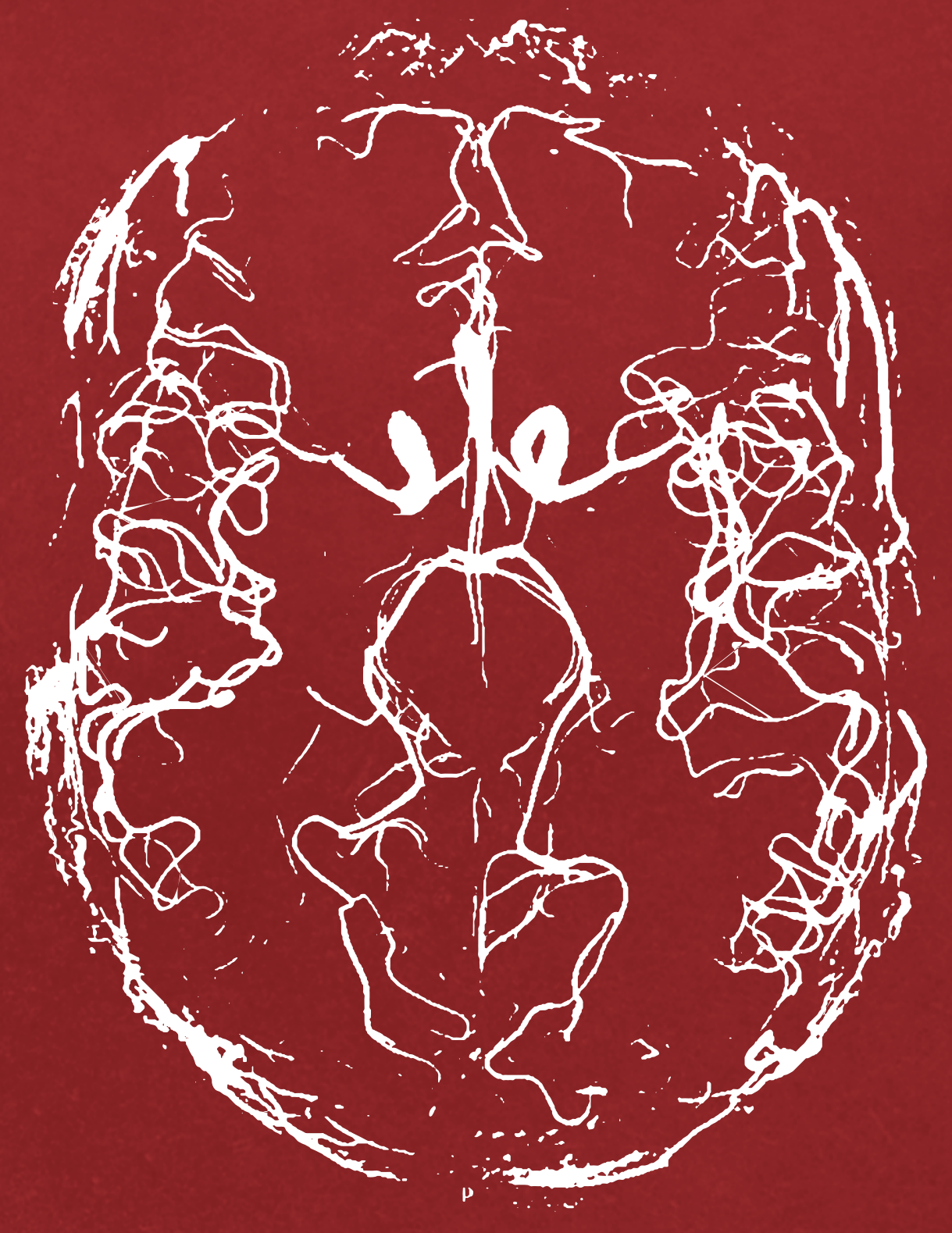




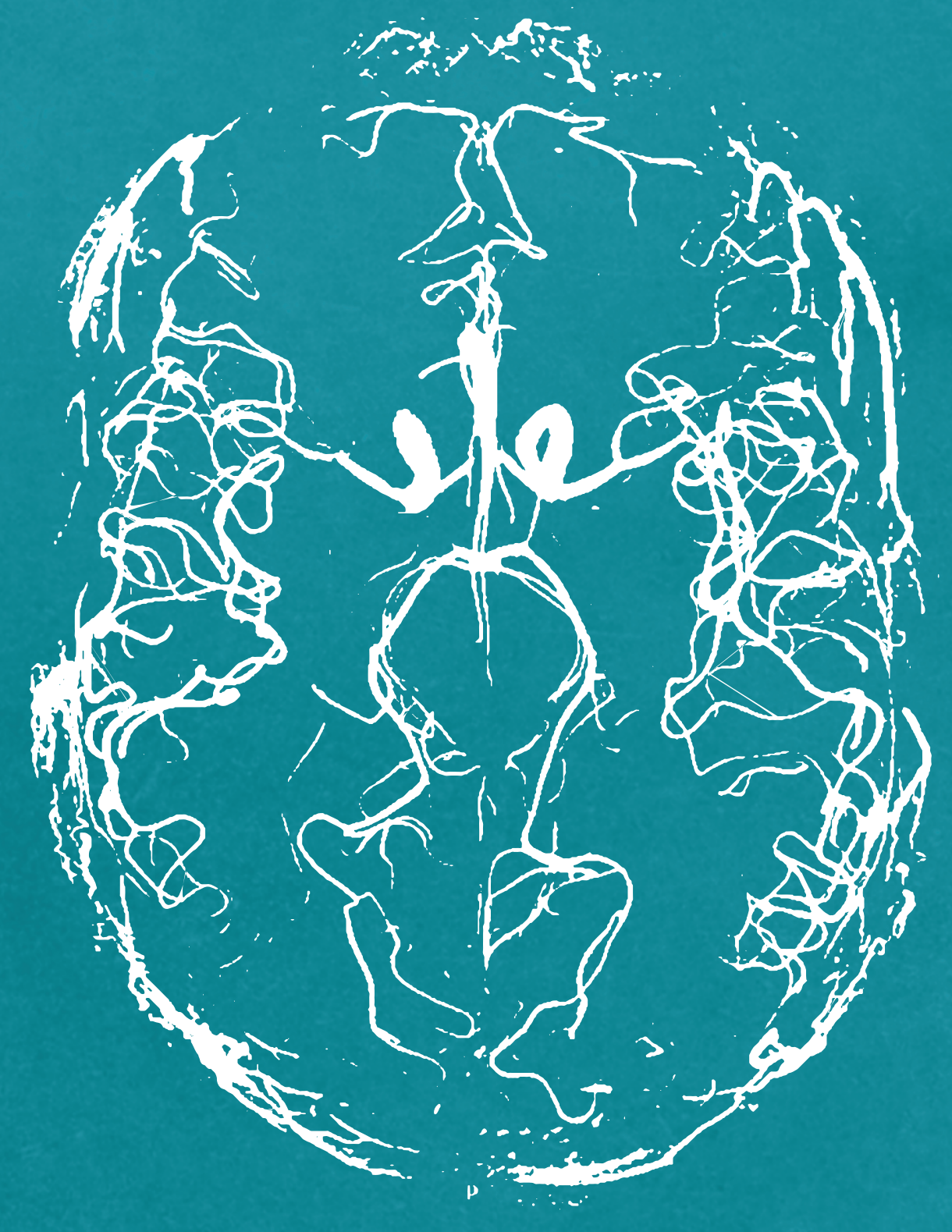




\section{CHAPTER}

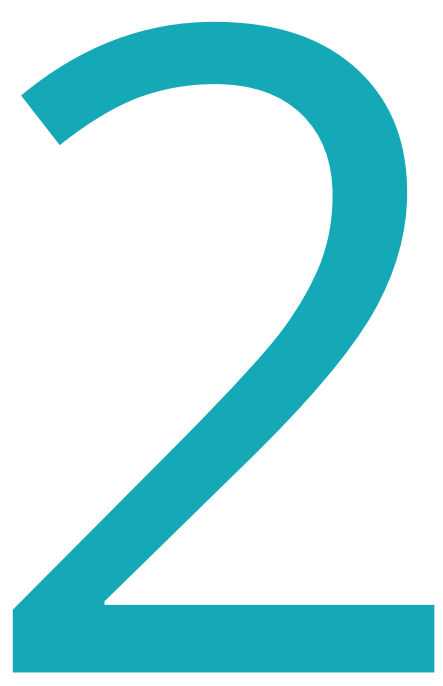

\section{Clinical Vascular Imaging in the Brain at 7T}

Laurens J.L. de Cocker ${ }^{1,2}$, Arjen Lindenholz' , Jaco J.M. Zwanenburg' ${ }^{1}$ Anja G. van der Kolk', Maarten Zwartbol' ${ }^{1}$, Peter R. Luijten ${ }^{1}$ and Jeroen Hendrikse ${ }^{1}$

${ }^{1}$ Department of Radiology, University Medical Center Utrecht, The Netherlands ${ }^{2}$ Department of Radiology, Kliniek Sint-Jan, Brussels, Belgium 


\begin{abstract}
Stroke and related cerebrovascular diseases are a major cause of mortality and disability. Even at standard-field-strengths (1.5T), MRI is by far the most sensitive imaging technique to detect acute brain infarctions and to characterize incidental cerebrovascular lesions, such as white matter hyperintensities, lacunes and microbleeds. Arterial time-of-flight (TOF) MR angiography (MRA) can depict luminal narrowing or occlusion of the major brain feeding arteries, and this without the need for contrast administration. Compared to 1.5T MRA, the use of high-field strength (3T) and even more so ultra-high-field strengths (7T), enables the visualization of the lumen of much smaller intracranial vessels, while adding a contrast agent to TOF-MRA at 7T may enable the visualization of even more distal arteries in addition to veins and venules. Moreover, with $3 \mathrm{~T}$ and 7T, the arterial vessel walls beyond the circle of Willis become visible with high-resolution vessel wall imaging. In addition, with 7T MRI, the brain parenchyma can now be visualized on a submillimeter scale. As a result, high-resolution imaging studies of the brain and its blood supply at 7T have generated new concepts of different cerebrovascular diseases. In the current article, we will discuss emerging clinical applications and future directions of vascular imaging in the brain at 7T MRI.
\end{abstract}




\section{Introduction}

Vascular disorders of the brain, including stroke, are a major cause of death in addition to physical and cognitive disability. During the past decades, imaging has become an indispensable tool in the work-up, treatment planning and follow-up of ischemic and hemorrhagic stroke, as well as in the identification of cerebrovascular anomalies predisposing to stroke. In addition, imaging may record the burden of incidental cerebrovascular lesions that may lead to pathologic brain aging. Compared to $\mathrm{CT}$ and other imaging modalities, brain magnetic resonance imaging (MRI) is by far the best technique to assess the total extent of cerebrovascular diseases in individual patients, as it allows for the accurate visualization of acute and chronic manifestations of large- and small-vessel disease in both the supra- and infra-tentorial regions. Although routine clinical practice is currently still limited to standard (1.5T) and high-field (3T) MRI, cerebrovascular disease evaluation at ultrahigh-field (7T) MRI may benefit from a high signal to noise ratio (SNR) which can be transferred into high spatial resolution, as well as a high contrast to noise ratio (CNR). By enabling the evaluation of the brain parenchyma on a submillimeter scale, very small cerebrovascular lesions, such as cortical microinfarcts, have come within the detection limit of $7 \mathrm{~T}$ and to a lesser degree 3T MRI. ${ }^{1}$ Also, compared to $1.5 \mathrm{~T}$ arterial MR angiography (MRA), the use of high-field (3T) and even more so ultra-high field MRI (7T), has enabled the visualization of the lumen of much more peripheral intracranial vessels, and of the intracranial vessel walls of the circle of Willis (CoW) and beyond. ${ }^{2,3}$ Finally, MR perfusion weighted imaging (PWI) may benefit from 7T, for instance by the increased susceptibility effects and the lower amount of contrast agent required for dynamic susceptibility contrast (DSC) perfusion at 7T. Thus, the advent of 7T has during the past decade resulted in a wave of research exploring new developments in cerebrovascular imaging, which are now increasingly finding their way into clinical practice. In the current article, we will review the emerging clinical applications and future directions of vascular imaging in the brain at 7T.

\section{Clinical applications}

A clinically feasible stroke imaging protocol at 7T has already been proposed and investigated for subacute and chronic stroke patients. ${ }^{4}$ This imaging protocol includes T1-weighted 3D Magnetization-Prepared Rapid-Acquired Gradient-Echo (3D-MPRAGE), T2-weighted 2D Fluid Attenuated Inversion Recovery (2D-FLAIR), T2-weighted 2D Turbo Spin Echo (2D-T2-TSE), T2*-weighted 2D Fast Low Angle Shot Gradient Echo (2D-HemoFLASH) and 3D arterial Time-of-Flight (TOF) MRA. However, the imaging protocol excludes diffusion weighted imaging (DWI), the most sensitive imaging technique to detect acute infarctions. ${ }^{4}$ With improvements being made to DWI at 7T, it may be expected that clinical stroke protocols at $7 \mathrm{~T}$ imaging will be extended to include acute stroke patients as well. 


\section{Ischemic stroke}

About $80 \%$ of strokes are ischemic and about $20 \%$ hemorrhagic in origin. Hemorrhagic transformation is a not infrequent complication of ischemic stroke and Figure 1 shows an axial 7T FLAIR image of a patient with ischemic stroke undergoing hemorrhagic transformation in the territory of the right middle cerebral artery. The figure displays the high degree of detail discernible with 7T in stroke patients. ${ }^{4}$ Most ischemic strokes have an extracranial origin and result either from cardio-embolism or artery-to-artery embolism from the carotid or vertebrobasilar arteries. Screening for cardiac arrhythmia and evaluation of the neck vessels may point towards the correct stroke origin in most of these patients. In case of artery-to-artery embolism from the neck, luminal imaging may reveal arterial stenosis, most frequently at the level of the carotid bifurcation.

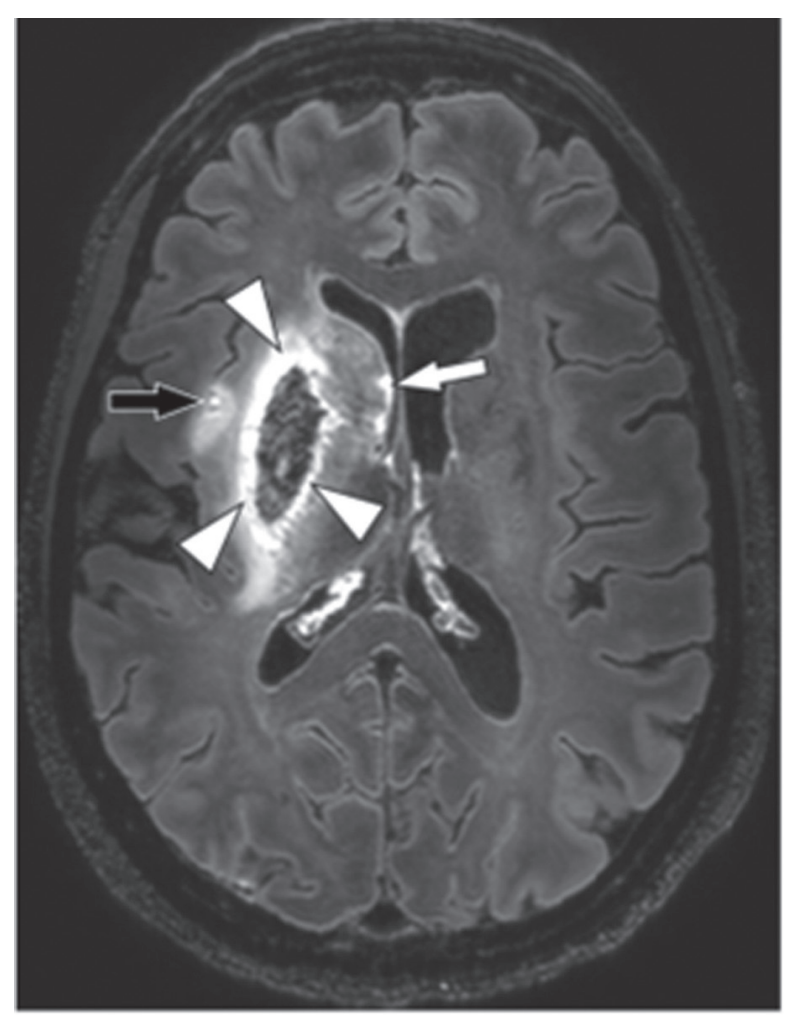

Figure 1. Ultra-high resolution imaging of ischemic stroke. Axial 7T contrast-enhanced 3D-FLAIR image of 50-year-old female with a recent right-sided ischemic stroke with hemorrhagic transformation, repetition time $8000 \mathrm{~ms}$, echo time $300 \mathrm{~ms}$, inversion time $2200 \mathrm{~ms}$, acquired voxel size $0.8 \times 0.8 \times 0.8 \mathrm{~mm}^{3}$, reconstructed voxel size $0.5 \times 0.5 \times 0.5 \mathrm{~mm}^{3}$, field-of-view $250 \times 250 \times 190 \mathrm{~mm}^{3}$, scan duration 10 minutes 48 seconds. A hyperintense border is seen to surround the lentiform nucleus (putamen and globus pallidus, arrowheads), which appears hypointense due to blood components. A smaller infarct is also seen in the insular region (black arrow), and multiple hyperintense dots are also seen along the ventricular border of the head of the caudate nucleus (white arrow), all of which are compatible with satellite infarctions. 
In a considerable proportion of patients, however, the origin of stroke may be related to intracranial arterial lesions, evaluation of which may especially benefit from high-resolution MR imaging. Not unlike arterial lesions in the neck, intracranial arterial lesions include atherosclerotic plaques, and, more rarely, dissection or vasculitis.

\section{Intracranial atherosclerosis}

Arterial TOF-MRA is routinely performed in stroke patients to detect intracranial arterial stenosis, and is usually preferred above angiographic techniques (CTA and DSA) because of its non-invasiveness, the lack of ionizing radiation, and no need to administer a contrast medium (Figure 2). Also, it can be acquired in the same imaging session as DWI, the most sensitive technique to detect acute infarction. Compared to 1.5T and 3T, arterial TOF-MRA at 7T allows the visualization of much smaller intracranial arteries, such as the lenticulostriate arteries (Figure 2), while adding a contrast agent may enable the visualization of even more distal arteries in addition to veins and venules. ${ }^{2,4,5}$ The presence of intracranial arterial narrowing alone, however, does not necessarily correspond to a stroke origin; arterial stenosis may already have been present a long time before the onset of stroke, and may be compensated by adequate primary (circle of Willis) or secondary (leptomeningeal) collaterals ${ }^{6,7}$ In addition, atherosclerotic plaques may be present without luminal narrowing due to arterial remodeling. ${ }^{8}$ Because of the shortcomings of lumenography, there is a need for intracranial vessel wall imaging to link cerebral infarction with intracranial arterial lesions, such as symptomatic plaques, dissection or vasculitis. Because of the small caliber of intracranial arteries, a high SNR which can be transferred into high spatial resolution, as well as a high CNR are required for visualization of the pathologic vessel wall, and even more so for the healthy vessel wall (Figure 3). ${ }^{3}$ Since spatial resolution increases with field strength, (ultra-)high field imaging techniques are required to visualize wall thickening of arteries of the circle of Willis and beyond. ${ }^{3}$ Also, for optimal vessel wall visualization, signal suppression of the arterial lumen (black blood imaging techniques including double inversion recovery and techniques based on motion-sensitizing prepulses) is required for delineation of the inner vessel wall (Figure 3, A and B), while cerebrospinal fluid (CSF) suppression facilitates the demarcation of the outer vessel wall (Figure 3B), especially for the more peripheral vessels surrounded by subarachnoid (CSF) spaces in case of cerebral atrophy. ${ }^{3}$ However, acquisition of 3D isotropic sequences with high resolution and sufficient brain coverage result in relatively long scan times (Figure 3). ${ }^{3,9}$ Compared to 3T imaging, the increased signal-to-noise ratio (SNR) of 7T leads to an overall better vessel wall visibility, visualizes more atherosclerotic plaques, and thus offers the highest potential to identify the total burden of intracranial atherosclerosis. ${ }^{10,11}$ Recently, several studies investigating the relationship between intracranial vessel 
wall changes on 3T and 7T and brain infarction have been published. ${ }^{12-14}$ Although most studies have so far only been performed in a limited number of patients, the following preliminary conclusions may be drawn. Eccentric atherosclerotic lesions are most frequently detected and seem to be associated with a focal (short-segment) thickening pattern, while concentric plaques usually show a more diffuse (long-segment) thickening. ${ }^{14}$ Although contrast-enhancement of intracranial atherosclerotic plaques is frequently observed and has been linked to the vessels supplying the area of ischemic injury, it may as well appear in asymptomatic lesions. ${ }^{10,14-16}$ Since atherosclerotic lesions of the intracranial vasculature cannot be correlated with histopathology in living patients (unlike the carotid plaques, which may be surgically removed by endarterectomy), only postmortem quantitative MRI-pathologic correlation studies have been performed to compare plaque contents with plaque signal intensities on 3T and 7T on CoW. ${ }^{17-20}$ These have shown the promising result that different tissue components of advanced intracranial plaques have distinguishable relaxation times on ultra-highresolution quantitative MR imaging. $\mathrm{T} 2$ and $\mathrm{T} 2 *$ relaxation times at $3 \mathrm{~T}$, and $\mathrm{T} 1$ relaxation times at $7 \mathrm{~T}$, have shown the most differences among individual tissue components of intracranial plaques, including lipid, fibrous tissue, fibrous cap, calcifications, and the healthy vessel wall. ${ }^{17,18}$ Hence, the most promising method for distinguishing intracranial plaque components at 7T is T1-weighted imaging.

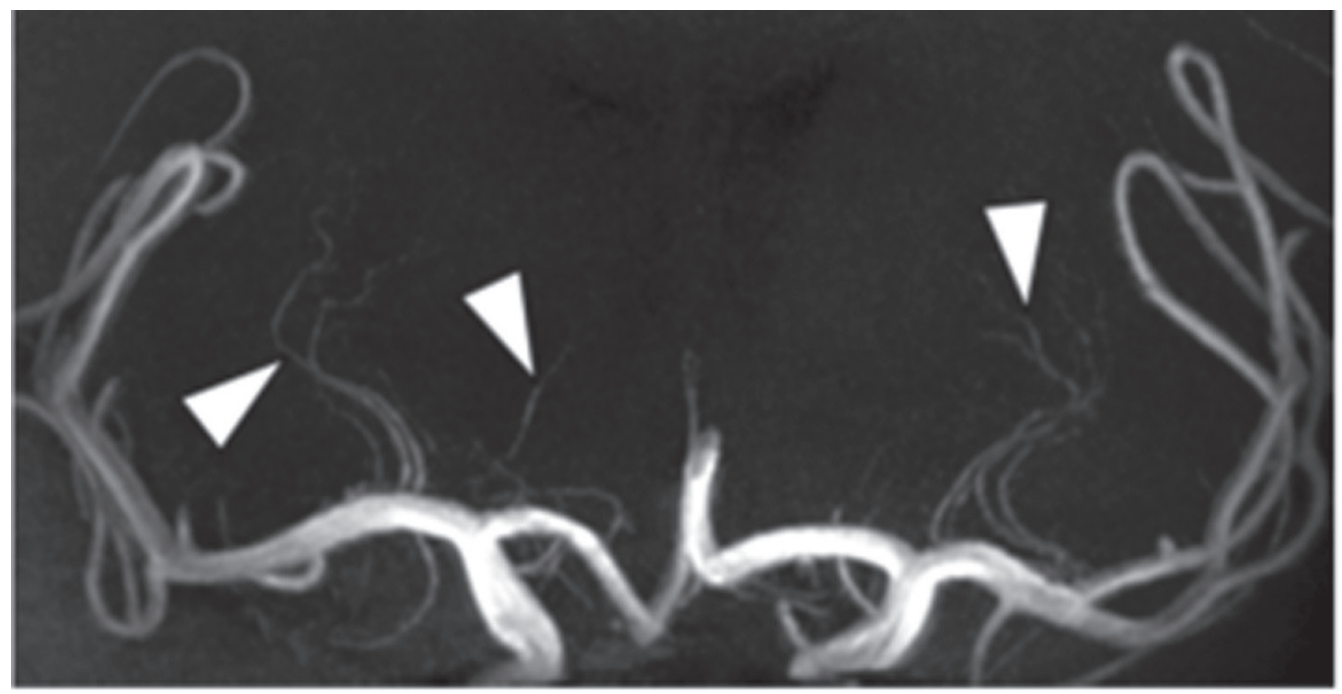

Figure 2. MR Angiography of the intracranial perforating arteries. Coronal Maximum Intensity Projection (MIP) of a 7T Time-of-Flight (TOF) MRA, repetition time $16 \mathrm{~ms}$, echo time $3.3 \mathrm{~ms}$, acquired voxel size $0.25 \times$ $0.3 \times 0.4 \mathrm{~mm}^{3}$, reconstructed voxel size $0.2 \times 0.2 \times 0.2 \mathrm{~mm}^{3}$, field-of-view $200 \times 190 \times 50 \mathrm{~mm}^{3}$, scan duration 9 minutes 54 seconds, performed in a 51-year-old male. Perforating lenticulostriate arteries (arrowheads) branching off from the middle cerebral arteries are clearly seen in both cerebral hemispheres. 


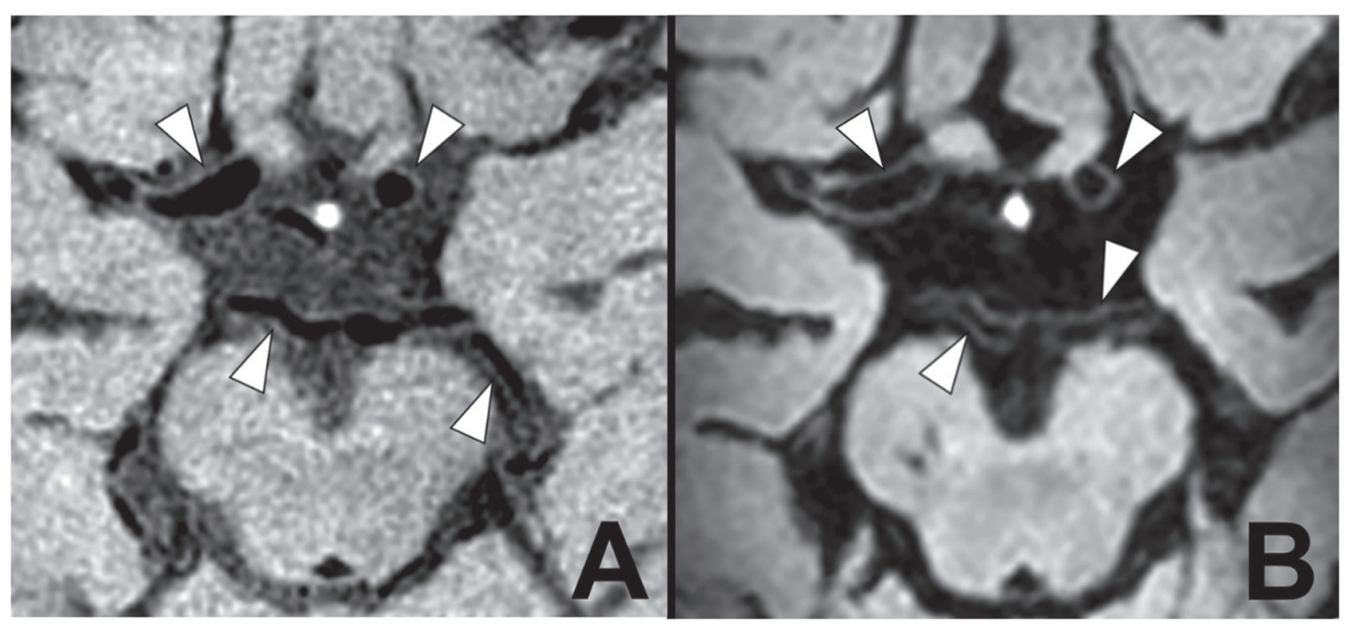

Figure 3. Intracranial vessel wall imaging at 3T and 7T. Intracranial vessel wall imaging of a 71-year-old male with a recent left sided ischemic infarction in the anterior circulation (not shown) resulting from symptomatic carotid artery disease. (A) A transverse 3T contrast-enhanced T1 Volume Isotropically Reconstructed Turbo Spin Echo Acquisition (VIRTA), repetition time $1500 \mathrm{~ms}$, echo time $36 \mathrm{~ms}$, acquired voxel size $0.6 \times 0.6 \times 1.0 \mathrm{~mm}^{3}$, reconstructed voxel size $0.5 \times 0.5 \times 0.5 \mathrm{~mm}^{3}$, field-of-view $200 \times 167 \times 45$ $\mathrm{mm}^{3}$, scan duration 6 minutes 42 seconds. ${ }^{14}$ Most of the arterial vessel walls of the circle of Willis are visible and appear to be normal (arrowheads). Blood is more suppressed than cerebrospinal fluid. (B) A transverse 7T post-contrast T1 Magnetization Preparation Inversion Recovery (MPIR) TSE acquisition, repetition time $3952 \mathrm{~ms}$, echo time $37 \mathrm{~ms}$, inversion time $1375 \mathrm{~ms}$, acquired voxel size $0.8 \times 0.8 \times 0.8 \mathrm{~mm}^{3}$, reconstructed voxel size $0.5 \times 0.5 \times 0.5 \mathrm{~mm}^{3}$, field-of-view $250 \times 250 \times 190 \mathrm{~mm}^{3}$, scan time 10 minutes 40 seconds. 9,21 The arterial vessel walls (arrowheads) are better seen due to an improved contrast with blood and cerebrospinal fluid, which is almost completely suppressed.

Intracranial dissection may be hard to diagnose due to the small caliber of the involved arteries, and thus its diagnosis may highly benefit from the spontaneous bright signal on T1 high-resolution vessel wall images, as has already been investigated at 3T. ${ }^{16,22}$ In addition, dissection often shows wall enhancement, and may present a visible flap and dual lumen. ${ }^{16,22}$

\section{Vasculitis, reversible vasoconstriction syndrome and moyamoya}

Central nervous system (CNS) vasculitis and reversible vasoconstriction syndrome (RCVS) often show clinical and lumenographic overlap, and at times only highresolution vessel wall imaging with MRI may show distinguishing features between these two entities. ${ }^{23,24}$ CNS vasculitis is characterized by short segments of vessel wall thickening, which is concentric more often than eccentric, and is associated with vessel wall enhancement, which may resolve after healing. ${ }^{16,23-25}$ Compared to CNS vasculitis, RCVS shows longer segments of reversible wall thickening, continuous throughout the entire wall of the diseased vessel, with no or only mild enhancement. ${ }^{24}$ Furthermore, high-resolution intracranial vessel wall imaging at $3 T$ has been reported to be beneficial in differentiating moyamoya disease, 
atherosclerotic-moyamoya syndrome, and vasculitic-moyamoya syndrome. ${ }^{26}$ Although vessel wall imaging studies at 7T for moyamoya are still lacking, MPRAGE has already been found superior to TOF-MRA at 7T due to shorter scanning times and better brain coverage. ${ }^{27}$

\section{Incidental or silent infarction and the aging brain}

Apart from brain infarction presenting with stroke (Figure 1), many (small) brain infarctions present with only few or non-specific clinical symptoms, or may even be clinically. ${ }^{28-30}$ Still, these infarcts may present later on as an incidental finding on neuroimaging studies. They are associated with cognitive decline and worse physical functioning, and an increased risk of future stroke..$^{31-33}$ Traditionally, incidental cerebral infarctions include large and small cortical infarcts as well as subcortical infarcts, of which the latter includes lacunar infarcts. ${ }^{34-37}$

\section{Lacunar infarcts, perivascular spaces and white matter hyperintensities}

Due to the superior evaluation of small-caliber arteries in the brain on 7T arterial TOF-MRA (Figure 2), it has been found that the number of lenticulostriate arteries supplying the basal ganglia is reduced in patients with lacunes (of presumed vascular origin) compared to age-matched controls. This finding has later been translated to $1.5 \mathrm{~T}$ with flow-sensitive black blood MRA, suggesting that occlusion of lenticulostriate arteries underlies lacunar infarction of the basal ganglia. 5,37,38 At times, lacunes may be difficult to distinguish from enlarged perivascular spaces (PVS), although the latter do not have a T2-hyperintense rim around the fluidfilled space on T2-weighted or FLAIR imaging, unless they traverse an area of white matter hyperintensity. ${ }^{37}$ At high resolution, a central vessel can occasionally be seen in the center of a perivascular space, which may differentiate the spaces from lacunes. ${ }^{37,39}$ Also, optimized MRI parameters and segmentation methods have been developed for PVS at 7T, which have shown that PVS are much more abundant than previously reported in young patients. ${ }^{39,40}$ Despite the term lacunar infarction, it has been shown that only a small proportion of lacunar infarcts progress to lacunes, ${ }^{41,42}$ and non-cavitating lacunar infarcts often continue to resemble white matter lesions instead. ${ }^{37,41}$ Also, in non-vascular disease such as multiple sclerosis (MS), characterization of white matter lesions with high resolution MR imaging has shown diagnostic benefits. With the increased SNR of 7T MRI, the typical localization of MS plaques around small venules can be demonstrated. ${ }^{43-45}$ 


\section{Cortical microinfarcts}

Another major clinical advancement of 7T MRI is its ability to detect cortical microinfarcts (CMIs) (Figure 4). Historically being a strictly pathologic diagnosis recorded during autopsy in elderly people, high resolution MRI has made it possible to visualize some of these lesions in vivo at $7 \mathrm{~T}$ (Figure 4$)^{46-49}$ and to a lesser degree at 3T. 1,50 Nevertheless, the majority of microinfarcts currently remain under the detection limits of clinical in vivo MRI. ${ }^{51}$ On post-mortem 7T MRI studies, three types of CMIs have been distinguished based on the involvement of all three cortical layers (type 1), two cortical layers (type 2), or one (superficial, middle or deep) cortical layer only (type 3.52 CMls should be distinguished from enlarged or atypically shaped perivascular spaces, which can be CMI mimics but which are located juxtacortically (Figure 5)..$^{53}$ CMls are associated with atherosclerosis, and are believed to be of microembolic origin. ${ }^{54-56}$ Also, CMIs are a new marker of vascular dementia, especially if occurring in strategic locations, such as the inferior frontal and cingulate gyri. ${ }^{50,52,57}$ Although CMIs have also been described in the cerebellum on post-mortem 7T MRI studies, the somewhat larger cortical infarct cavities $(<1.5 \mathrm{~cm})$ are more frequently observed in that region (Figure 6). ${ }^{58-60}$ As originally found on post-mortem $7 \mathrm{~T} \mathrm{MRI}$, those cerebellar cortical infarct cavities demonstrate a characteristic sparing of juxtacortical white matter (Figure 6), a finding which has recently allowed translation to lower field-strength MRI in vivo. ${ }^{59,61}$ Like CMIs, cerebellar cortical infarct cavities are associated with atherosclerosis and believed to be of embolic origin. ${ }^{30,33}$
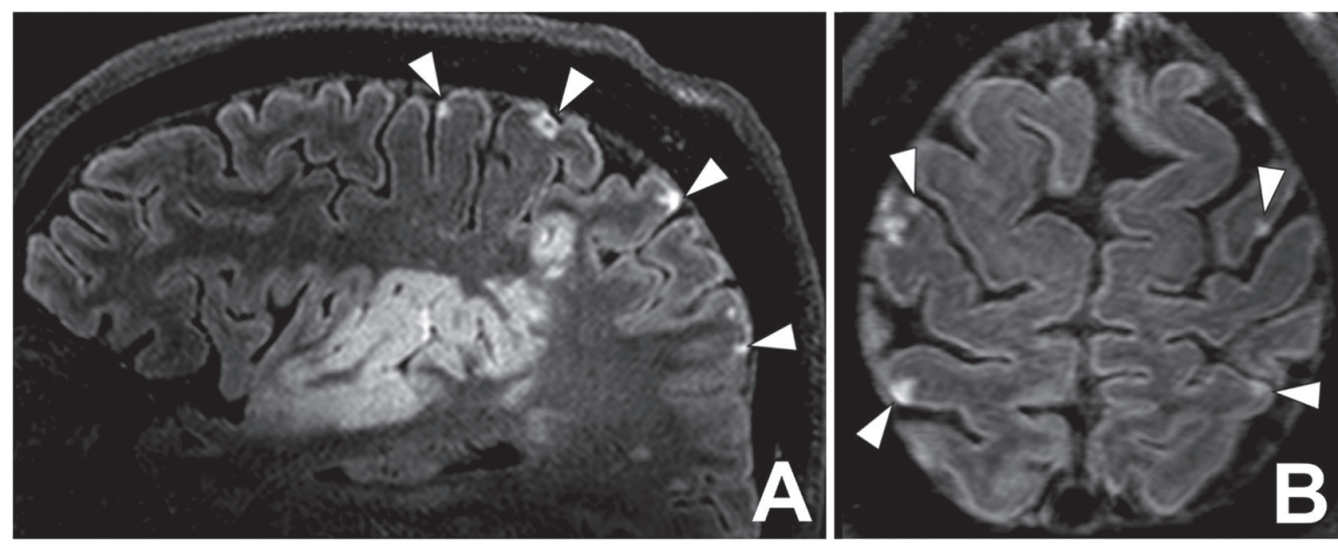

Figure 4. Cortical microinfarcts. 7T contrast-enhanced 3D-FLAIR imaging of a 68-year-old man with a large right-sided temporoparietal ischemic infarction (A), repetition time $8000 \mathrm{~ms}$, echo time $300 \mathrm{ms,}$ inversion time $2200 \mathrm{~ms}$, acquired voxel size $0.8 \times 0.8 \times 0.8 \mathrm{~mm}^{3}$, reconstructed voxel size $0.5 \times 0.5 \times 0.5$ $\mathrm{mm}^{3}$, field-of-view $250 \times 250 \times 190 \mathrm{~mm}^{3}$, scan duration 10 minutes 48 seconds. (A) Sagittal and (B) axial reconstruction shows multiple tiny cortical hyperintensities, compatible with cortical microinfarcts. Most cortical microinfarcts seen involve all cortical layers, compatible with type I microinfarcts according to. ${ }^{52}$ 

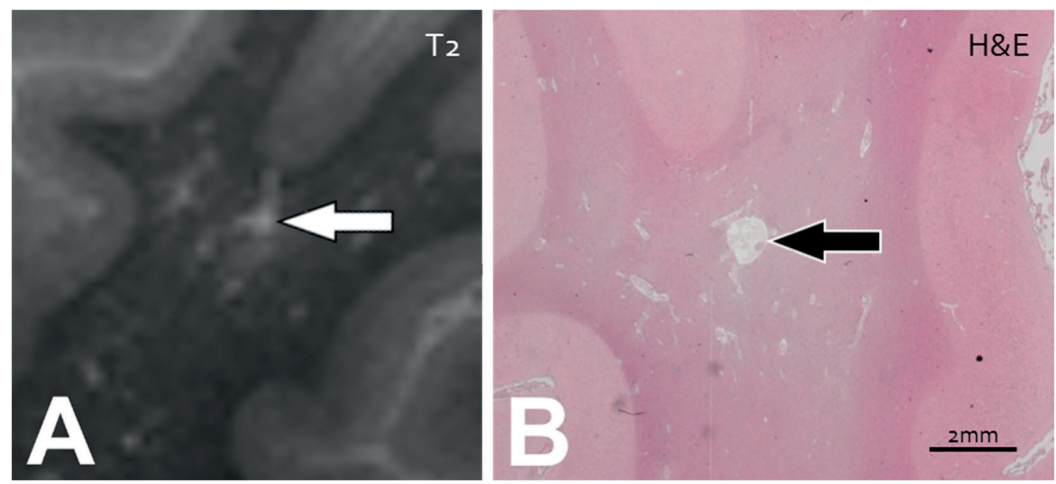

Figure 5. Perivascular Spaces and Lacunar Infarcts. An example of a juxtacortical, enlarged perivascular space (PVS) mimicking a cerebral microinfarct (CMI), in a post-mortem brain of a 68-year-old female with Alzheimer's Disease pathology (BB VI) and severe cerebral amyloid angiopathy, identified on (A) T2weighted ex vivo MR-imaging (repetition time $3500 \mathrm{~ms}$, echo time $164 \mathrm{~ms}$, acquired voxel size $0.4 \times 0.4 \times$ $0.4 \mathrm{~mm}^{3}$, no SENSE acceleration, scan duration 112 minutes) and with (B) histopathological correlation, Hematoxylin\&Eosin (H\&E) staining. (A) The small hyperintense enlarged PVS is located in juxtaposition to the cortex (white arrow). (B) No evidence of neuronal death or gliosis is seen on H\&E (black arrow). (images courtesy of S.J. van Veluw).

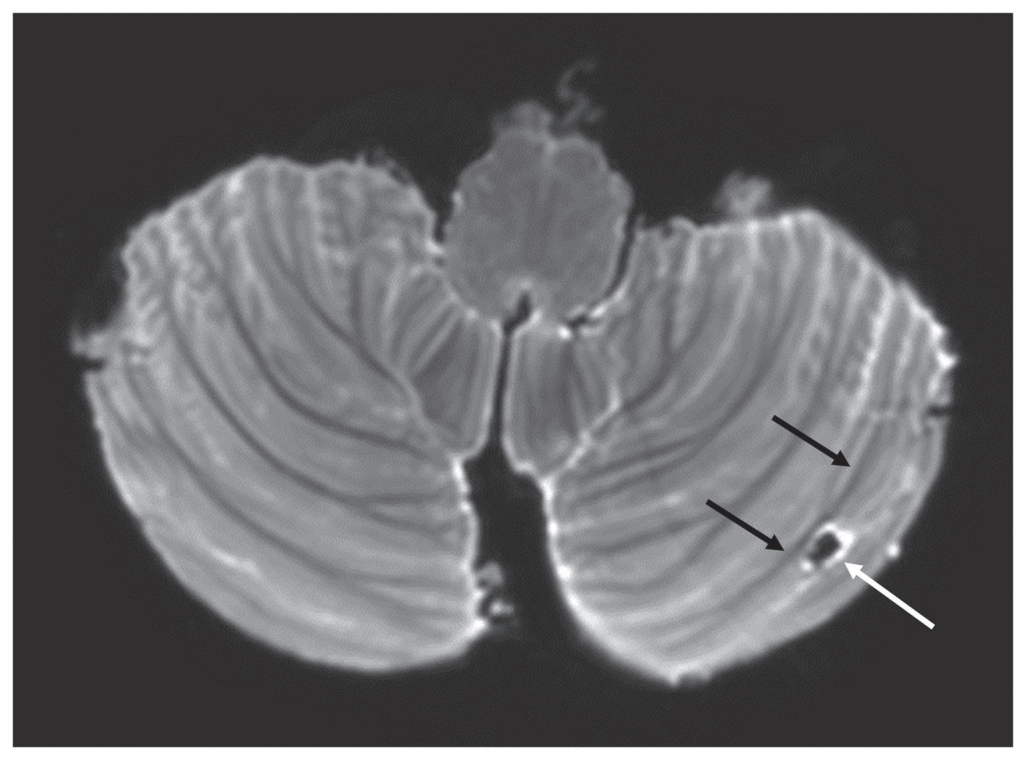

Figure 6. Cerebellar cortical infarct cavity on 7T post-mortem MRI. Cerebellar cortical infarct cavity (white arrow) in the left cerebellar hemisphere on T2-weighted 7T post-mortem MRI; 3D TSE; TR 3000 ms; TE $207 \mathrm{~ms}$; reduced focusing angle of $40^{\circ}$; acquired voxel size $0.70 \times 0.70 \times 0.70 \mathrm{~mm}^{3}$; matrix size $284 \times$ 169; FOV $200 \times 119 \times 120 \mathrm{~mm}^{3}$; SENSE: $2 \times 2$; scan duration 8 minutes 39 seconds. The cavity and the surroundings of the cerebellum are black due to Fomblin, a proton-free fluid without MR signal. Notice the sharp demarcation of the cavity and surrounding hyperintense gliosis (white arrow) from the intact subjacent white matter (black arrows), which proved to be characteristic imaging features of cerebellar cortical infarct cavities and enabled the translation to clinical 1.5T MRI scans. ${ }^{59,61}$ 


\section{Microbleeds}

Detection of microbleeds may be of potential clinical relevance for a variety of pathologies, including brain trauma, hypertensive microangiopathy, cerebral amyloid angiopathy (CAA) and Alzheimer's dementia (AD). ${ }^{37,62-67}$ A recent postmortem study has shown a strong association between cerebral microbleeds and CMIs in CAA, suggesting a shared underlying pathophysiologic mechanism. ${ }^{68}$ Susceptibility effects and the sensitivity of T2* and SWI for detecting microbleeds increase with field strength, and 3D dual-echo T2*-weighted imaging at $7 \mathrm{~T}$ has been found to result in better and more reliable detection of microbleeds compared with 3D T2*-weighted imaging at $1.5 \mathrm{~T}$ in vivo. ${ }^{69}$ Interestingly, a recent combined in-vivo/post-mortem MRI correlation study has shown that microbleeds on in vivo MRI are specific for microhemorrhages in CAA, but that increasing the resolution of magnetic resonance images with ultra-high resolution post mortem 7T MRI (<100 $\mu \mathrm{m}$ isotropic voxels) results in the detection of more 'nonhemorrhagic' pathology. ${ }^{51}$ In addition, diagnostic confidence of rating microbleeds in vivo has not been found significantly higher at $7 \mathrm{~T}$ compared to $3 \mathrm{~T}$ for the diagnosis and exclusion of microbleeds and vascular malformations. ${ }^{70}$ Visual rating of microbleeds is more challenging at $7 \mathrm{~T}$ than at lower field strengths due to the increased susceptibility effects of adjacent structures, such as veins..$^{71,72}$ Because manual detection of microbleeds may be time consuming with observer variability, automated detection systems of microbleeds have been developed..$^{73-75}$

\section{Aneurysms, arteriovenous malformations and cavernomas}

\section{Cerebral aneurysms}

7T MRI has also been proven beneficial for the depiction of cerebral aneurysms, with overall image quality equaling the gold standard DSA. ${ }^{76} 7 \mathrm{~T}$ has already proven its superiority over $1.5 \mathrm{~T}$ in the analysis of aneurysm neck and dome, as well as in the evaluation of aneurysm location at the parent vessel. ${ }^{77}$ Using $7 \mathrm{~T}$ TOF-MRA, microaneurysms with diameters under $1 \mathrm{~mm}$ are now being described, e.g. ventricular microaneurysms in patients with moyamoya disease. ${ }^{78} 7 \mathrm{~T}$ MPRAGE has been found superior to 7T TOF-MRA for the assessment of aneurysm features, with less artifacts and simultaneous high quality assessment of the brain with full coverage. ${ }^{77}$ Interestingly, high-resolution vessel wall imaging has shown a strong association between ruptured aneurysms and circumferential aneurysm wall enhancement in subarachnoid hemorrhage $(\mathrm{SAH})$, which may identify the site of rupture in patients with multiple intracranial aneurysms. ${ }^{79-82}$ In addition, circumferential wall enhancement has been more frequently observed in unruptured but unstable (growing and symptomatic) aneurysms..$^{80,81}$ Although a minority of incidentally discovered aneurysms also show wall enhancement, it still needs to be established if these are at an increased risk of rupture. ${ }^{80}$ Apart 
from aneurysm vessel wall enhancement, regional differences in wall thickness of unruptured aneurysms have been demonstrated on 7T MRI, and thinner regions have been associated with regions of higher wall shear stress determined with phase-contrast MRI. ${ }^{83,84}$ Future studies still need to address if variations in wall thickness are associated with risk of aneurysm rupture.

\section{Arteriovenous malformations and cavernomas}

Although DSA is still considered the gold standard for assessment of brain arteriovenous malformations (AVMs), arterial TOF-MRA has become a non-invasive alternative for detection and follow-up of AVMs. ${ }^{76,80}$ In a recent comparative study with 1.5T arterial TOF-MRA and DSA, non-contrast-enhanced 7T MPRAGE as well as 7T arterial TOF-MRA have been found superior to 1.5T arterial TOF-MRA in the delineation of the nidus, feeder(s), draining vein(s), and in the relationship between AVMs and adjacent vessels (non-feeding vessels relevant for surgical treatment). ${ }^{85}$ Compared to non-enhanced arterial TOF-MRA, MPRAGE offers a very high-quality of adjacent brain structures, but is less performant in the visualization of draining veins, probably due to saturation effects of slow flowing arterialized venous blood. ${ }^{85}$ Although overall image quality at 7 T equals DSA, all described MRA techniques at 7T still miss dynamic information about the blood flow patterns within the AVM. ${ }^{85}$ This may be overcome with phase contrast 4D flow imaging, which is a currently rapidly developing field and also possible at 7T with higher SNR than at 3T. ${ }^{86}$ Future studies will still need to assess the diagnostic potential for treatment follow-up of brain AVMs as hemorrhagic changes or artefacts due to embolic agents or clips could be misinterpreted as residual flow. ${ }^{85} \mathrm{Also}$, the role of high-resolution vessel-wall MRI for brain AVMs still needs to be established.

Finally, 7T MRI significantly improves the visualization of cavernomas and associated developmental venous anomalies (DVA) due to a higher spatial resolution and susceptibility sensitivity. ${ }^{87-89}$

\section{Future directions}

Different experimental and functional techniques are currently being developed and tested to assess cerebrovascular function with 7T MRI on a microvascular level. ${ }^{72}$ Amongst others, these include 3D phase contrast (PC)-MRA and blood oxygenation level dependent (BOLD) functional MRI (fMRI). 7T PC-MRA is a promising technique to visualize and measure blood flow and pulsatility, for example of the CoW and lenticulostriate arteries .90,91 Functional assessment with 3D PC-MRI may better discriminate between healthy and diseased arteries/ arterioles compared to anatomical imaging alone, and may as such be applied to assess the relationship between white matter hyperintensities and lacunes on the 
one hand and blood flow in the perforating arteries on the other hand. ${ }^{91,92}$ BOLD fMRI, which is typically used for task-related and resting state fMRI experiments, may be adapted to measure cerebral perfusion and cerebrovascular reactivity (CVR) before and after a certain vascular challenge, such as the inhalation of carbon dioxide, breath-holding or medication administration (acetazolamide). ${ }^{72}$ These stimuli all result in an increased blood flow to the brain and a decreased BOLD signal, and it is generally assumed that brain regions with a decreased signal change upon a certain challenge have a lower cerebrovascular reserve and are at an increased risk of future ischemia. ${ }^{72}$ At 7T, BOLD fMRI may detect differences in cerebrovascular reactivity at a millimeter scale, and may be applied to study the association of CVR with lacunes, white matter hyperintensities and microbleeds. ${ }^{93-96}$

Despite all technical advancements, 7T still faces some practical challenges. First, technical improvements are needed to counter the increased magnetic field (B0) and radiofrequency field (B1) inhomogeneities (Figure 7). These inhomogeneities cause signal loss and a variable flip angle, most prominently in the temporal (Figure 7) and cerebellar regions, which not only hinders the evaluation of these regions in individual subjects, but also hampers group analyses of $7 \mathrm{~T}$ datasets. The use of dielectric pads may partly overcome this problem. Upgraded head and neck coil technology with larger coverage and more homogenous magnetic fields are required for arterial spin labeling (ASL) perfusion imaging, which at present is still preferentially performed at $3 T .^{72}$ Whole brain diffusion MRI also is still challenging at 7T due to magnetic field inhomogeneities, shorter relaxation times, and increased power deposition (specific absorption rate (SAR)), although progress is currently being made to acquire high-quality and reproducible DWI and diffusion tensor imaging (DTI) datasets., ${ }^{4,97-99}$ Finally, promising results on implant safety - which has long been a major drawback for clinical application have shown no increased risk of radiofrequency heating of metallic implants. ${ }^{100}$ These safety results and the growing evidence of an additional clinical value of $7 \mathrm{~T}$ MRI will pave the way towards certification for (regular) clinical applications in the near future. ${ }^{4}$ 


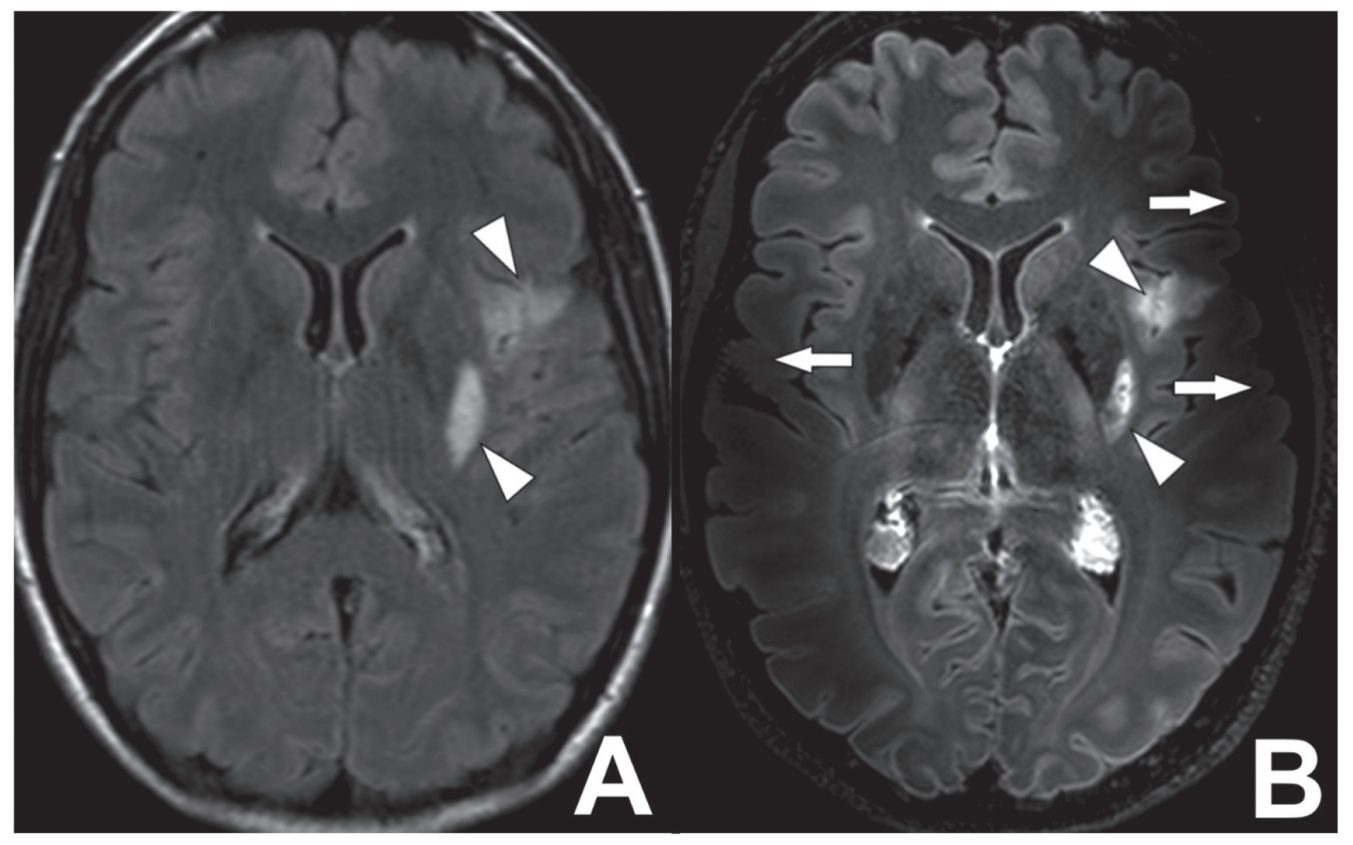

Figure 7. B1-inhomogeneity artifacts on a 7T MR FLAIR images of a 42-year-old female with a recent left-sided ischemic stroke. (A) A transverse 3T FLAIR image, repetition time $10000 \mathrm{~ms}$, echo time $120 \mathrm{ms,}$ inversion time $2800 \mathrm{~ms}$, acquired voxel size $0.75 \times 1.27 \times 4.00 \mathrm{~mm}^{3}$, reconstructed voxel size $0.4 \times 0.4 \times 4.0$ $\mathrm{mm}^{3}$, field-of-view $230 \times 182 \times 129 \mathrm{~mm}^{3}$, scan duration 2:00 minutes. No B0- or B1-inhomogeneity artifacts are seen on the image. Some patient motion is present in the image. Two hyperintense lesions are present; along the posterior margin of the left putamen and in the left insular region (white arrowheads). (B) A transverse 7T FLAIR image, repetition time $8000 \mathrm{~ms}$, echo time $300 \mathrm{~ms}$, inversion time $2200 \mathrm{~ms}$, acquired voxel size $0.8 \times 0.8 \times 0.8 \mathrm{~mm}^{3}$, reconstructed voxel size $0.5 \times 0.5 \times 0.5 \mathrm{~mm}^{3}$, field-of-view $250 \times$ $250 \times 190 \mathrm{~mm}^{3}$, scan time 10 minutes 48 seconds. The image is affected by B1-imhomogeniteity artefacts, best seen in both temporal lobes (white arrows). The two hyperintense lesions are seen in much more detail compared to the 3T MR image in (A) (white arrowheads).

\section{Conclusion}

We described the added value and emerging applications of 7T MRI in the clinical evaluation of cerebrovascular disorders. 7T MRI has been proven superior to lower field-strength MRI for the evaluation of the intracranial vessel lumen and vessel walls, and allows to study the brain parenchyma for microvascular pathology on a submillimeter scale. 


\section{References}

1. Van Dalen JW, Scuric EE, van Veluw SJ, et al. Cortical microinfarcts detected in vivo on 3 Tesla MRI: clinical and radiological correlates. Stroke 2015;46:255-7.

2. Harteveld AA, De Cocker LJ, Dieleman N, et al. High-resolution postcontrast time-of-flight MR angiography of intracranial perforators at 7.0 Tesla. PloS one 2015;10:e0121051.

3. Dieleman N, van der Kolk AG, Zwanenburg JJ, et al. Imaging intracranial vessel wall pathology with magnetic resonance imaging: current prospects and future directions. Circulation 2014;130:192-201.

4. Madai VI, von Samson-Himmelstjerna FC, Bauer M, et al. Ultrahigh-field MRI in human ischemic stroke-a 7 tesla study. PloS one 2012;7:e37631.

5. Kang CK, Park CA, Park CW, Lee YB, Cho ZH, Kim YB. Lenticulostriate arteries in chronic stroke patients visualised by $7 \mathrm{~T}$ magnetic resonance angiography. International Journal of Stroke 2010;5:374-80.

6. Liebeskind DS, Cotsonis GA, Saver JL, et al. Collateral circulation in symptomatic intracranial atherosclerosis. Journal of Cerebral Blood Flow and Metabolism 2011;31:1293-301.

7. Hartkamp NS, Hendrikse J, De Cocker LJ, de Borst GJ, Kappelle LJ, Bokkers RP. Misinterpretation of ischaemic infarct location in relationship to the cerebrovascular territories. Journal of Neurology, Neurosurgery, and Psychiatry 2016;87:1084-90.

8. Qiao Y, Anwar Z, Intrapiromkul J, et al. Patterns and Implications of Intracranial Arterial Remodeling in Stroke Patients. Stroke 2016;47:434-40.

9. Van der Kolk AG, Zwanenburg J, Brundel M, et al. Intracranial vessel wall imaging at 7.0-T MRI. Stroke 2011;42:2478-84.

10. Harteveld AA, van der Kolk AG, van der Worp HB, et al. High-resolution intracranial vessel wall MRI in an elderly asymptomatic population: comparison of 3T and 7T. European Radiology 2017;27:1585-1595.

11. Zhu $C$, Haraldsson $H$, Tian $B$, et al. High resolution imaging of the intracranial vessel wall at 3 and 7 T using 3D fast spin echo MRI. MAGMA 2016;29:559-70.

12. Dieleman N, van der Kolk AG, van Veluw SJ, et al. Patterns of intracranial vessel wall changes in relation to ischemic infarcts. Neurology 2014;83:1316-20.

13. Dieleman N, van der Kolk AG, Zwanenburg J, et al. Relations between location and type of intracranial atherosclerosis and parenchymal damage. Journal of Cerebral Blood Flow and Metabolism 2016;36:1271-80.

14. Dieleman N, Yang W, Abrigo JM, et al. Magnetic Resonance Imaging of Plaque Morphology, Burden, and Distribution in Patients With Symptomatic Middle Cerebral Artery Stenosis. Stroke 2016; 47:1797-1802.

15. Aoki S, Shirouzu I, Sasaki Y, et al. Enhancement of the intracranial arterial wall at MR imaging: relationship to cerebral atherosclerosis. Radiology 1995;194:477-81.

16. Swartz RH, Bhuta SS, Farb RI, et al. Intracranial arterial wall imaging using high-resolution 3-tesla contrast-enhanced MRI. Neurology 2009;72:627-34. 
17. Harteveld AA, Denswil NP, Siero JC, et al. Quantitative Intracranial Atherosclerotic Plaque Characterization at 7T MRI: An Ex Vivo Study with Histologic Validation. AJNR American Journal of Neuroradiology 2016;37:802-10.

18. Jiang Y, Zhu C, Peng W, et al. Ex-vivo imaging and plaque type classification of intracranial atherosclerotic plaque using high resolution MRI. Atherosclerosis 2016;249:10-6.

19. Majidi S, Sein J, Watanabe M, et al. Intracranial-derived atherosclerosis assessment: an in vitro comparison between virtual histology by intravascular ultrasonography, 7T MRI, and histopathologic findings. AJNR American Journal of Neuroradiology 2013;34:2259-64.

20. Van der Kolk AG, Zwanenburg J, Denswil NP, et al. Imaging the intracranial atherosclerotic vessel wall using 7T MRI: initial comparison with histopathology. AJNR American Journal of Neuroradiology 2015;36:694-701.

21. Van der Kolk AG, Hendrikse J, Brundel M, Biessels GJ, Smit EJ, Visser F. Multi-sequence whole-brain intracranial vessel wall imaging at 7.0 tesla. European radiology 2013;23:2996-3004.

22. Arai D, Satow T, Komuro T, Kobayashi A, Nagata H, Miyamoto S. Evaluation of the Arterial Wall in Vertebrobasilar Artery Dissection Using High-Resolution Magnetic Resonance Vessel Wall Imaging. Journal of Stroke and Cerebrovascular Diseases 2016;25:1444-50.

23. Mandell DM, Matouk CC, Farb RI, Krings T, Agid R, Brugge K. Vessel wall MRI to differentiate between reversible cerebral vasoconstriction syndrome and central nervous system vasculitis: preliminary results. Stroke 2012;43:860-2.

24. Obusez EC, Hui F, Hajj-Ali RA, Cerejo R, Calabrese LH, Hammad T. High-resolution MRI vessel wall imaging: spatial and temporal patterns of reversible cerebral vasoconstriction syndrome and central nervous system vasculitis. AJNR 2014;35:1527-32.

25. Kuker W, Gaertner S, Nagele T, Dopfer C, Schoning M, Fiehler J. Vessel wall contrast enhancement: a diagnostic sign of cerebral vasculitis. Cerebrovasc Dis 2008;26:23-9.

26. Mossa-Basha M, de Havenon A, Becker $\mathrm{KJ}$, et al. Added Value of Vessel Wall Magnetic Resonance Imaging in the Differentiation of Moyamoya Vasculopathies in a Non-Asian Cohort. Stroke 2016;47:1782-8.

27. Dengler NF, Madai VI, Wuerfel J, et al. Moyamoya Vessel Pathology Imaged by UltraHigh-Field Magnetic Resonance Imaging at 7.0 T. Journal of Stroke and Cerebrovascular Diseases 2016;25:1544-51.

28. Saini M, Ikram K, Hilal S, Qiu A, Venketasubramanian N, Chen C. Silent stroke: not listened to rather than silent. Stroke 2012;43:3102-4.

29. Fanning JP, Wesley AJ, Wong AA, Fraser JF. Emerging spectra of silent brain infarction. Stroke 2014;45:3461-71.

30. De Cocker LJ, Compter A, Kappelle LJ, Luijten PR, Hendrikse J, Van der Worp HB. Cerebellar cortical infarct cavities and vertebral artery disease. Neuroradiology 2016;58:853-7.

31. Vermeer SE, Prins ND, den Heijer T, Hofman A, Koudstaal PJ, Breteler MM. Silent brain infarcts and the risk of dementia and cognitive decline. The New England Journal of Medicine 2003;348:1215-22. 
32. Bernick C, Kuller L, Dulberg C, et al. Silent MRI infarcts and the risk of future stroke: the cardiovascular health study. Neurology 2001;57:1222-9.

33. De Cocker LJ, Kloppenborg RP, van der Graaf Y, et al. Cerebellar Cortical Infarct Cavities: Correlation With Risk Factors and MRI Markers of Cerebrovascular Disease. Stroke 2015;46:3154-60.

34. Riba-Llena I, Koek M, Verhaaren BF, et al. Small cortical infarcts: prevalence, determinants, and cognitive correlates in the general population. International journal of stroke : official journal of the International Stroke Society 2015;10 Suppl A100:18-24.

35. Longstreth WT, Jr., Bernick C, Manolio TA, Bryan N, Jungreis CA, Price TR. Lacunar infarcts defined by magnetic resonance imaging of 3660 elderly people: the Cardiovascular Health Study. Archives of Neurology 1998;55:1217-25.

36. Del Bene A, Makin SD, Doubal FN, Inzitari D, Wardlaw JM. Variation in risk factors for recent small subcortical infarcts with infarct size, shape, and location. Stroke 2013;44:3000-6.

37. Wardlaw JM, Smith EE, Biessels GJ, et al. Neuroimaging standards for research into small vessel disease and its contribution to ageing and neurodegeneration. The Lancet Neurology 2013;12:822-38.

38. Okuchi $S$, Okada $T$, Ihara $M$, et al. Visualization of lenticulostriate arteries by flowsensitive black-blood MR angiography on a 1.5 T MRI system: a comparative study between subjects with and without stroke. AJNR American Journal of Neuroradiology 2013;34:780-4.

39. Zong X, Park SH, Shen D, Lin W. Visualization of perivascular spaces in the human brain at 7T: sequence optimization and morphology characterization. Neurolmage 2016; 125:895-902.

40. Park SH, Zong X, Gao Y, Lin W, Shen D. Segmentation of perivascular spaces in 7T MR image using auto-context model with orientation-normalized features. Neurolmage 2016;134:223-35.

41. Potter GM, Doubal FN, Jackson CA, et al. Counting cavitating lacunes underestimates the burden of lacunar infarction. Stroke 2010;41:267-72.

42. Moreau F, Patel S, Lauzon ML, et al. Cavitation after acute symptomatic lacunar stroke depends on time, location, and MRI sequence. Stroke 2012;43:1837-42.

43. Ge Y, Zohrabian VM, Grossman RI. Seven-Tesla magnetic resonance imaging: new vision of microvascular abnormalities in multiple sclerosis. Archives of Neurology 2008;65:812-6.

44. Tallantyre EC, Brookes MJ, Dixon JE, Morgan PS, Evangelou N, Morris PG. Demonstrating the perivascular distribution of MS lesions in vivo with 7-Tesla MRI. Neurology 2008; 70:2076-8.

45. Gizewski ER, Monninghoff C, Forsting M. Perspectives of Ultra-High-Field MRI in Neuroradiology. Clinical Neuroradiology 2015;25 Suppl 2:267-73.

46. Zwanenburg JJ, Hendrikse J, Luijten PR. Generalized multiple-layer appearance of the cerebral cortex with 3D FLAIR 7.0-T MR imaging. Radiology 2012;262:995-1001. 
47. Van Veluw SJ, Zwanenburg J, Engelen-Lee J, et al. In vivo detection of cerebral cortical microinfarcts with high-resolution 7T MRI. Journal of Cerebral Blood Flow and Metabolism 2013;33:322-9.

48. Van Veluw SJ, Fracasso A, Visser F, et al. FLAIR images at 7 Tesla MRI highlight the ependyma and the outer layers of the cerebral cortex. Neurolmage 2015;104:100-9.

49. Fracasso A, van Veluw SJ, Visser F, et al. Lines of Baillarger in vivo and ex vivo: Myelin contrast across lamina at 7T MRI and histology. Neurolmage 2016;133:163-75.

50. Van Veluw SJ, Hilal S, Kuijf HJ, et al. Cortical microinfarcts on 3T MRI: Clinical correlates in memory-clinic patients. Alzheimer's \& Dementia 2015;11:1500-9.

51. Van Veluw SJ, Charidimou A, van der Kouwe AJ, et al. Microbleed and microinfarct detection in amyloid angiopathy: a high-resolution MRI-histopathology study. Brain 2016;139:3151-62.

52. De Reuck J, Deramecourt V, Auger F, et al. Post-mortem 7.0-tesla magnetic resonance study of cortical microinfarcts in neurodegenerative diseases and vascular dementia with neuropathological correlates. Journal of the Neurological Sciences 2014;346:85-9.

53. Van Veluw SJ, Zwanenburg J, Rozemuller AJ, Luijten PR, Spliet WG, Biessels GJ. The spectrum of MR detectable cortical microinfarcts: a classification study with 7-tesla postmortem MRI and histopathology. Journal of Cerebral Blood Flow and Metabolism 2015;35:676-83.

54. Zheng L, Vinters HV, Mack WJ, Zarow C, Ellis WG, Chui HC. Cerebral atherosclerosis is associated with cystic infarcts and microinfarcts but not Alzheimer pathologic changes. Stroke 2013;44:2835-41.

55. Raman MR, Preboske GM, Przybelski SA, et al. Antemortem MRI findings associated with microinfarcts at autopsy. Neurology 2014;82:1951-8.

56. De Rotte AA, Koning W, den Hartog AG, et al. 7.0 T MRI detection of cerebral microinfarcts in patients with a symptomatic high-grade carotid artery stenosis. Journal of Cerebral Blood Flow and Metabolism 2014;34:1715-9.

57. De Reuck JL, Auger F, Durieux N, et al. The Topography of Cortical Microinfarcts in Neurodegenerative Diseases and in Vascular Dementia: A Postmortem 7.0-Tesla Magnetic Resonance Imaging Study. European Neurology 2016;76:57-61.

58. De Reuck JL, Deramecourt V, Auger F, et al. The significance of cortical cerebellar microbleeds and microinfarcts in neurodegenerative and cerebrovascular diseases. A post-mortem 7.0-tesla magnetic resonance study with neuropathological correlates. Cerebrovasc Dis 2015;39:138-43.

59. De Cocker LJ, van Veluw SJ, Biessels GJ, et al. Ischaemic cavities in the cerebellum: an ex vivo 7-tesla MRI study with pathological correlation. Cerebrovasc Dis 2014;38:17-23.

60. De Cocker LJ, van Veluw SJ, Fowkes M, Luijten PR, Mali WP, Hendrikse J. Very small cerebellar infarcts: integration of recent insights into a functional topographic classification. Cerebrovasc Dis 2013;36:81-7.

61. De Cocker LJ, Geerlings MI, Hartkamp NS, et al. Cerebellar infarct patterns: The SMARTMedea study. Neurolmage Clinical 2015;8:314-21. 
62. Wardlaw JM, Smith C, Dichgans M. Mechanisms of sporadic cerebral small vessel disease: insights from neuroimaging. The Lancet Neurology 2013;12:483-97.

63. Akoudad S, Wolters FJ, Viswanathan A, et al. Association of Cerebral Microbleeds With Cognitive Decline and Dementia. JAMA neurology 2016;73:934-43.

64. Knudsen KA, Rosand J, Karluk D, Greenberg SM. Clinical diagnosis of cerebral amyloid angiopathy: validation of the Boston criteria. Neurology 2001;56:537-9.

65. Pontes-Neto OM, Auriel E, Greenberg SM. Advances in our Understanding of the Pathophysiology, Detection and Management of Cerebral Amyloid Angiopathy. Eur Neurol Rev 2012;7:134-9.

66. Brundel M, Reijmer YD, van Veluw SJ, et al. Cerebral microvascular lesions on highresolution 7-Tesla MRI in patients with type 2 diabetes. Diabetes 2014;63:3523-9.

67. Bian W, Hess CP, Chang SM, Nelson SJ, Lupo JM. Susceptibility-weighted MR imaging of radiation therapy-induced cerebral microbleeds in patients with glioma: a comparison between 3T and 7T. Neuroradiology 2014;56:91-6.

68. Van Veluw SJ, Biessels GJ, Klijn CJ, Rozemuller AJ. Heterogeneous histopathology of cortical microbleeds in cerebral amyloid angiopathy. Neurology 2016;86:867-71.

69. Conijn MM, Geerlings MI, Biessels G], et al. Cerebral microbleeds on MR imaging: comparison between 1.5 and 7T. AJNR American Journal of Neuroradiology 2011;32:1043-9.

70. Springer E, Dymerska B, Cardoso PL, et al. Comparison of Routine Brain Imaging at $3 \mathrm{~T}$ and 7 T. Investigative Radiology 2016;51:469-82.

71. de Bresser J, Brundel M, Conijn MM, et al. Visual cerebral microbleed detection on 7T MR imaging: reliability and effects of image processing. AJNR American Journal of Neuroradiology 2013;34:E61-4.

72. Harteveld AA, van der Kolk AG, Zwanenburg JJ, Luijten PR, Hendrikse J. 7-T MRI in Cerebrovascular Diseases: Challenges to Overcome and Initial Results. Topics in magnetic resonance imaging : TMRI 2016;25:89-100.

73. Kuijf HJ, de Bresser J, Geerlings MI, et al. Efficient detection of cerebral microbleeds on 7.0 T MR images using the radial symmetry transform. Neurolmage 2012;59:2266-73.

74. Kuijf HJ, Brundel M, de Bresser J, et al. Semi-Automated Detection of Cerebral Microbleeds on 3.0 T MR Images. PloS one 2013;8:e66610.

75. Van den Heuvel TL, van der Eerden AW, Manniesing R, et al. Automated detection of cerebral microbleeds in patients with Traumatic Brain Injury. Neurolmage Clinical 2016;12:241-51.

76. Wrede $\mathrm{KH}$, Matsushige T, Goericke SL, et al. Non-enhanced magnetic resonance imaging of unruptured intracranial aneurysms at 7 Tesla: Comparison with digital subtraction angiography. European Radiology 2017;27:354-64.

77. Wrede $\mathrm{KH}$, Dammann $\mathrm{P}$, Monninghoff $\mathrm{C}$, et al. Non-enhanced MR imaging of cerebral aneurysms: 7 Tesla versus 1.5 Tesla. PloS one 2014;9:e84562. 
78. Matsushige T, Kraemer M, Schlamann $M$, et al. Ventricular Microaneurysms in Moyamoya Angiopathy Visualized with 7T MR Angiography. AJNR American Journal of Neuroradiology 2016;37:1669-72.

79. Matouk CC, Mandell DM, Gunel M, Bulsara KR, Malhotra A, Hebert R. Vessel wall magnetic resonance imaging identifies the site of rupture in patients with multiple intracranial aneurysms: proof of principle. Neurosurgery 2013;72:492-6.

80. Matouk CC, Cord BJ, Yeung J, Malhotra A, Johnson MH, Minja FJ. High-resolution Vessel Wall Magnetic Resonance Imaging in Intracranial Aneurysms and Brain Arteriovenous Malformations. Topics in magnetic resonance imaging : TMRI 2016;25:49-55.

81. Edjlali M, Gentric JC, Regent-Rodriguez C, Trystram D, Hassen WB, Lion S. Does aneurysmal wall enhancement on vessel wall MRI help to distinguish stable from unstable intracranial aneurysms? Stroke 2014;45:3704-6.

82. Nagahata S, Nagahata M, Obara M, et al. Wall Enhancement of the Intracranial Aneurysms Revealed by Magnetic Resonance Vessel Wall Imaging Using Three-Dimensional Turbo Spin-Echo Sequence with Motion-Sensitized Driven-Equilibrium: A Sign of Ruptured Aneurysm? Clinical Neuroradiology 2016;26:277-83.

83. Kleinloog R, Korkmaz E, Zwanenburg JJ, et al. Visualization of the aneurysm wall: a 7.0-tesla magnetic resonance imaging study. Neurosurgery 2014;75:614-22.

84. Blankena R, Kleinloog R, Verweij BH, et al. Thinner Regions of Intracranial Aneurysm Wall Correlate with Regions of Higher Wall Shear Stress: A 7T MRI Study. AJNR American Journal of Neuroradiology 2016;37:1310-7.

85. Wrede KH, Dammann P, Johst $\mathrm{S}$, et al. Non-Enhanced MR Imaging of Cerebral Arteriovenous Malformations at 7 Tesla. European Radiology 2016;26:829-39.

86. Markl M, Schnell S, Wu C, et al. Advanced flow MRI: emerging techniques and applications. Clinical Radiology 2016;71:779-95.

87. Schlamann M, Maderwald S, Becker W, et al. Cerebral cavernous hemangiomas at 7 Tesla: initial experience. Academic Radiology 2010;17:3-6.

88. Dammann P, Barth M, Zhu Y, et al. Susceptibility weighted magnetic resonance imaging of cerebral cavernous malformations: prospects, drawbacks, and first experience at ultra-high field strength (7-Tesla) magnetic resonance imaging. Neurosurgical Focus 2010;29:E5.

89. Dammann P, Wrede $\mathrm{KH}$, Maderwald S, et al. The venous angioarchitecture of sporadic cerebral cavernous malformations: a susceptibility weighted imaging study at 7 T MRI. Journal of Neurology, Neurosurgery, and Psychiatry 2013;84:194-200.

90. Van Ooij P, Zwanenburg JJ, Visser F, et al. Quantification and visualization of flow in the Circle of Willis: time-resolved three-dimensional phase contrast MRI at $7 \mathrm{~T}$ compared with 3 T. Magnetic Resonance in Medicine 2013;69:868-76.

91. Kang CK, Park CA, Lee DS, et al. Velocity measurement of microvessels using phasecontrast magnetic resonance angiography at 7 Tesla MRI. Magnetic Resonance in Medicine 2016;75:1640-6. 
92. Bouvy WH, Geurts LJ, Kuijf HJ, et al. Assessment of blood flow velocity and pulsatility in cerebral perforating arteries with 7-T quantitative flow MRI. NMR in biomedicine 2016;29:1295-304.

93. Conijn MM, Hoogduin JM, van der Graaf Y, Hendrikse J, Luijten PR, Geerlings MI. Microbleeds, lacunar infarcts, white matter lesions and cerebrovascular reactivity - a $7 \mathrm{~T}$ study. Neurolmage 2012;59:950-6.

94. Siero JC, Hermes D, Hoogduin H, Luijten PR, Ramsey NF, Petridou N. BOLD matches neuronal activity at the $\mathrm{mm}$ scale: a combined $7 \mathrm{~T}$ fMRI and ECoG study in human sensorimotor cortex. Neurolmage 2014;101:177-84.

95. Bhogal AA, Siero JC, Fisher JA, et al. Investigating the non-linearity of the BOLD cerebrovascular reactivity response to targeted hypo/hypercapnia at 7T. Neurolmage 2014;98:296-305.

96. Bhogal AA, Philippens ME, Siero JC, et al. Examining the regional and cerebral depthdependent BOLD cerebrovascular reactivity response at 7T. Neurolmage 2015;114:23948.

97. Vu AT, Auerbach E, Lenglet $C$, et al. High resolution whole brain diffusion imaging at $7 T$ for the Human Connectome Project. Neurolmage 2015;122:318-31.

98. Andersson JLR, Sotiropoulos SN. An integrated approach to correction for off-resonance effects and subject movement in diffusion MR imaging. Neurolmage 2016;125:1063-78.

99. Sotiropoulos SN, Hernandez-Fernandez M, Vu AT, et al. Fusion in diffusion MRI for improved fibre orientation estimation: An application to the 3T and 7T data of the Human Connectome Project. Neurolmage 2016;134:396-409.

100. Kraff O, Wrede KH, Schoemberg T, et al. MR safety assessment of potential RF heating from cranial fixation plates at 7 T. Medical Physics 2013;40:042302. 


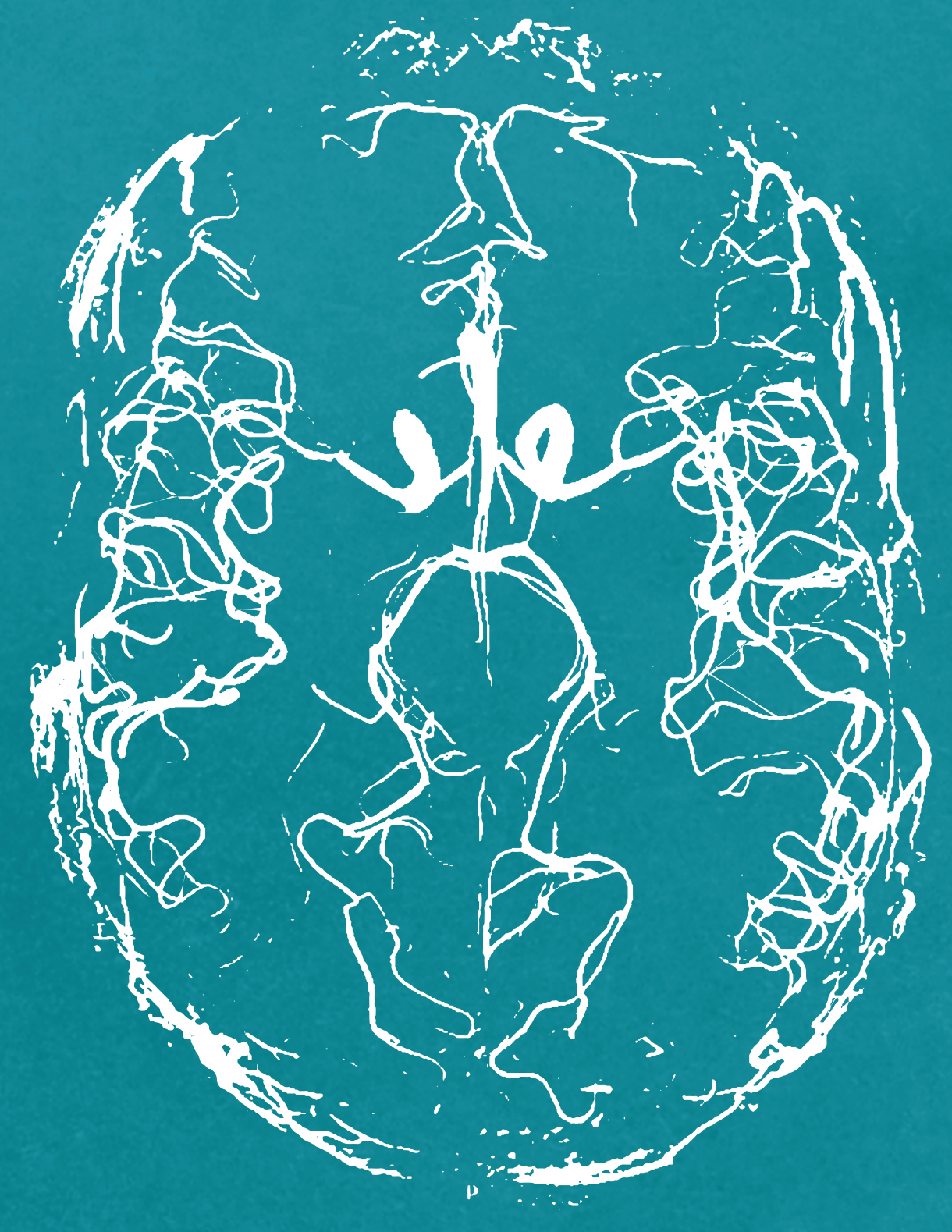




\section{CHAPTER}

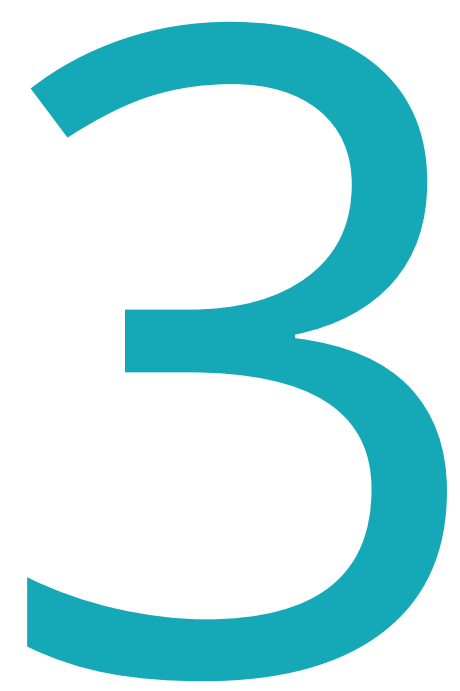

\section{The Use and Pitfalls of Intracranial Vessel Wall Imaging: How We do It}

Arjen Lindenholz' , Anja G. van der Kolk1', Jaco J. M. Zwanenburg ${ }^{1}$ and Jeroen Hendrikse ${ }^{1}$

${ }^{1}$ Department of Radiology, University Medical Center Utrecht, The Netherlands Published in Radiology, 2018; 286 (1) 12-28. 


\begin{abstract}
Intracranial vessel wall magnetic resonance imaging (MRI) has gained much attention in the past decade, and has become part of state-of-the-art MRI protocols to assist in diagnosing the cause of ischemic stroke. With intracranial vessel wall MRI, vessel wall characteristics have tentatively been described for atherosclerosis, vasculitis, dissections, moyamoya disease and aneurysms. With the increasing demand and subsequently increased use of intracranial vessel wall MRI in clinical practice, radiologists should be aware of the choices in imaging parameters and how they affect image quality, the clinical indications, methods of assessment and limitations in the interpretation of these images. In this 'How I do It' article, the authors will discuss the technical requirements and considerations for vessel wall image acquisition in general, describe their own vessel wall imaging protocol at 3 tesla ( $\mathrm{T}$ ) and 7T, show a step-by-step basic assessment of intracranial vessel wall imaging as performed at their institution - including commonly encountered artifacts and pitfalls - and summarize the commonly reported imaging characteristics of various intracranial vessel wall diseases for direct clinical applicability. Finally, future technical and clinical considerations for full implementation of intracranial vessel wall MRI in clinical practice, including the need for histological validation and acquisition time reduction, will be discussed.
\end{abstract}




\section{Introduction}

In recent years, intracranial vessel wall magnetic resonance imaging (MRI) has seen an exponential increase in popularity and clinical applicability. ${ }^{1,2}$ Several years ago, vessel wall imaging was restricted to extracranial (peripheral) arteries, such as the carotid arteries, in which atherosclerotic 'vulnerable plaques' - prone to causing embolism and subsequent ischemic stroke - could be assessed using different dedicated MRI sequences. However, increasing evidence shows that not extracranial but intracranial atherosclerosis (ICAS) is the leading causes of ischemic stroke worldwide. $^{3-6}$ In addition, ICAS has been associated with an increased (recurrent) stroke risk and vascular dementia. ${ }^{4,-9}$ This change of insight into causes of ischemic stroke has led to a trend towards imaging the intracranial vasculature.

Traditional imaging methods for visualizing the intracranial arteries include intraarterial digital subtraction angiography (DSA), computed tomography angiography (CT/CTA), Doppler ultrasonography (US) and magnetic resonance angiography (MRA). DSA can detect stenotic lesions in large, as well as small arteries, including the A2 segment of the anterior cerebral artery, M2-M3 segments of the middle cerebral artery (MCA), P2 segment of the posterior cerebral artery, and even more peripheral segments of the intracranial arteries. With unenhanced CT arterial vessel wall calcifications can be detected that are associated with future stroke risk. ${ }^{10-13}$ CTA is an easily accessible and fast procedure in patients with acute stroke and can depict stenotic lesions very accurately in the more proximal intracranial arteries and its branches. ${ }^{14}$ Doppler ultrasonography can depict the vessel wall of primarily the middle cerebral artery (MCA), thereby providing (single-artery restricted) information on vessel wall pathology. Finally contrast materialenhanced and time-of-flight MRA, like CTA, can depict stenotic lesions in proximal cerebral arteries, less quickly but without additional radiation dose compared with CTA. However, all of these techniques - except for Doppler US - have the disadvantage of only depicting the arterial lumen (including possible stenoses) and not the culprit vessel wall itself. Intracranial vessel wall MRI has two major advantages over DSA, CTA and MRA: I. It can depict non-stenotic lesions; and II. It can further characterize stenotic lesions that have already been detected with common angiographic methods.

In this 'How I do It' article, we describe the utility and practical steps for performing and interpreting intracranial vessel wall MRI scans. These practical steps are based on our teaching experience of intracranial vessel wall MRI to radiologists and neuroradiologists who have started using this method in daily clinical practice. The key questions that are often raised during their learning curve will also be addressed in this article. Key steps and common vessel wall pathology will be shown in illustrative figures of patient examples. 


\section{The imaging protocol - What do we need?}

\section{Vessel wall MRI sequence prerequisites}

For intracranial vessel wall MRI, both a high contrast-to-noise ratio (CNR) and a high spatial resolution are needed to visualize the thin arterial vessel wall and to characterize vessel wall lesions.,15-19 CNR in vessel wall imaging comprises signal contrast of the vessel wall relative to its direct surroundings, that is, blood and cerebrospinal fluid (CSF). It is dependent on both sequence parameters and magnetic field strength, where CNR generally increases with increasing field strength. The achievable spatial resolution is also dependent on the field strength, as well as on acquisition time. Finally, the different image contrast weightings need to be considered when developing a vessel wall MRI protocol. ${ }^{1}$

\section{Vessel wall CNR}

The first step in achieving a high vessel wall CNR is to suppress the luminal blood. Most intracranial vessel wall MRI sequences rely on the intrinsic "black blood" properties of three-dimensional (3D) turbo spin-echo pulse sequences (TSE) with variable flip-angle refocusing pulses. ${ }^{17,19-23}$ In these sequences, black blood is achieved by intravoxel dephasing of flowing blood, which is most effective when low flip-angle refocusing pulses are used. ${ }^{17,24}$ Alternatively, preparation pulses can be used to obtain blood suppression, such as double inversion recovery, ${ }^{25}$ motionsensitizing preparation pulses, ${ }^{26}$ or delay alternating with nutation for tailored excitation (DANTE) preparation pulses. ${ }^{20}$ Adding preparation pulses generally increases acquisition time. Currently, there is a need for a thorough analysis, both theoretically and experimentally, assessing the performance of the various blood suppression techniques in the presence of slow flow (directly next to the vessel wall).

For optimal CNR, not only suppression of blood signal to depict the inner boundary of the vessel wall, but also suppression of CSF signal to depict the outer boundary is essential, especially when outward vascular remodeling is present. ${ }^{27-30}$ At $3 T$, the CNR between vessel wall and lumen is superior to CNR between vessel wall and CSF on most pulse sequences. Because an inversion recovery pulse as a CSF suppressing technique is both time-consuming and detrimental to the vessel wall signal-to-noise ratio (SNR), these vessel wall MRI sequences rely implicitly (without using CSF suppression preparation pulses) or explicitly (using Delay Alternating with Nutation for Tailored Excitation; DANTE) on CSF flow for CSF suppression. 19-21,31,32 Consequently, suppression of CSF will be less in compartments where there is a slower flow of CSF, for example around the vessel wall and at locations where there is little CSF surrounding the vessels. However, a recent promising development for improving CSF suppression at $3 \mathrm{~T}$ is the incorporation of an anti-driven-equilibrium (ADE) pulse, which relies on $T_{1}$ and $T_{2}$ relaxation properties and is independent 
of CSF flow. ${ }^{32,33}$ At higher field strengths, such as 7T, the higher attainable SNR enables the use of previously mentioned inversion recovery pulse, resulting in nearly optimal CSF suppression. ${ }^{15}$

\section{Spatial resolution}

Due to a lack of in vivo - ex vivo correlation studies (see chapter 'Future Prospects'), it is currently not clear what minimum spatial resolution is necessary to accurately diagnose intracranial vessel wall pathology, nor is it known what spatial coverage is clinically relevant. Previous histopathologic studies have shown the intracranial arterial vessel wall to vary in thickness from 0.2-0.4 mm for the distal internal carotid artery (ICA) to $0.2-0.3 \mathrm{~mm}$ for the middle cerebral artery. ${ }^{34}$ However, radiological measurements have shown a larger vessel wall thickness of approximately 1.0 $\mathrm{mm}$ for both middle cerebral artery and internal carotid artery. ${ }^{35}$ Several reasons can account for this difference, for example underestimation in histopathologic studies due to preparation techniques (cell shrinkage (8-20\%) in histopathologic preparation), ${ }^{36,37}$ or overestimation in the radiological studies due to partial volume effects (relatively large voxels), measurement errors and/or pulsatility effects of the vessel wall during acquisition. On the basis of these considerations, and given the fact that one needs at least two voxels in an object to measure its size/ thickness accurately, ${ }^{38}$ an MRI sequence with $0.18 \mathrm{~mm}$ isotropic voxels - assuming a histologically processed wall thickness of $0.3 \mathrm{~mm}$ and a mean shrinkage effect of $15 \%$ - would theoretically enable highly detailed assessment of the circle of Willis and, depending on the field of view (FOV), its large and small branches. ${ }^{34}$ However, it remains to be seen whether this much detail is really necessary in clinical practice in all patient groups. ${ }^{39}$ Also, (ultra) high resolution sequences would be met with significant time constraints, limiting their application to cooperative patients with low morbidity, or necessitating either introduction of very fast imaging techniques or compromising between spatial resolution and FOV.

To avoid these limitations, intracranial vessel wall MRI sequences in current practice are mainly aimed at detecting larger lesions in the intracranial arteries proximal and just distal to the circle of Willis (M1, A1, P1 segments) and at the border of the M1-M2, A1-A2 and P1-P2 segments. The acquired in-plane spatial resolution of these sequences ranges between $0.4 \times 0.4 \mathrm{~mm}^{2}$ to $0.9 \times 0.9 \mathrm{~mm}^{2} .1,18,28,31,35,40,41$ For more distal arteries, however, detection reliability will decrease because the diameter of these arteries and therefore lesions at the walls of these arteries become smaller, increasing the impact of partial volume effects, ${ }^{35}$ which needs to be taken into account when assessing the vessel walls in clinical practice (see 'Systematic approach of vessel wall assessment'). 


\section{Isotropic versus anisotropic voxels}

A related question is whether and/or when to use isotropic or anisotropic voxels. Most experts in the field of vessel wall MRI sequence development prefer sequences with isotropic voxels, which render multiplanar assessment more feasible. Compared with, for example, the carotid artery, intracranial arteries are often tortuous and have varying orientations, making multiplanar reconstruction an important asset in assessment of these arteries. In anisotropic sequences, a very high in-plane spatial resolution can be achieved within reasonable acquisition times, enabling detailed assessment of vessel wall lesions and atherosclerotic plaque characterization when the FOV is placed perpendicular to the lesion. However, due to the larger through-plane voxel size, small lesions are subject to partial volume effects. Therefore, it may well be dependent on the specific clinical question on a single-patient base which type of sequence (isotropic versus anisotropic voxels) to use. For instance, a radiologist may consider isotropic sequences in patients with no previous imaging or known vessel wall lesion as a method of screening the intracranial arteries, while an anisotropic sequence could be used to assess a known lesion.

The advantage of interpreting vessel wall MR images in multiple planes is currently not supported by much research data. The results of recent studies suggest that the use of transverse images alone may be sufficient for the interpretation of vessel wall MR images. ${ }^{42,43}$ In one of these studies, 3D sequences with a high inplane spatial resolution and a larger slice thickness were either performed in the transverse plane or perpendicular to the MCA. Vessel wall lesions of the MCA were also detected on the images that were acquired non-perpendicular to the artery.

\section{$2 D$ versus $3 D$}

To increase in-plane spatial resolution, 2D spin-echo sequences can be used as an alternative to $3 \mathrm{D}$ methods. ${ }^{1,28,31,44}$ The $2 \mathrm{D}$ in-plane spatial resolution is equivalent to $3 \mathrm{D}$, and volume averaging errors due to thicker sections ( $2 \mathrm{~mm}$, no gap) are alleviated by repeating the sequences in sagittal and/or axial planes targeted to a lesion in question. Sequence acquisition times are short (approximately 3 minutes) with good SNR. The drawbacks of these 2D spin-echo methods are the need to target a specific lesion due to limited coverage in order to keep imaging times short, and the increased dependence on correct positioning of the FOV. Two-dimensional methods can therefore best be applied in cases in which there is a known intracranial stenosis on which the FOV can be focused, and has highest value in characterizing the underlying pathological process (e.g., atherosclerotic plaque characterization). 


\section{Field strength}

A further consideration is what field strength to use. In our experience and that of others, ${ }^{2}$ 1.5T does not achieve sufficient SNR and CNR within a reasonable acquisition time. Therefore, a field strength of 3T or higher is mandatory. In light of the increased availability of 3T MRI platforms, this makes intracranial vessel wall MRI feasible in many hospitals worldwide. The advantage of a 7T field strength is an increased SNR and CNR compared with 3T, which enables better intrinsic image contrast and a higher spatial resolution within reasonable acquisition times. $^{16,18}$ Specifically for vessel wall MRI, the higher SNR also enables the use of the inversion recovery pulse mentioned above to completely suppress CSF, and a large FOV for whole-brain coverage; however, this does come at the cost of a lower spatial resolution to keep acquisition times within limits. Nonetheless, although current 7T vessel wall MRI pulse sequences have a lower or comparable spatial resolution compared with published (and our own) 3T sequences, visualization of the vessel wall was still found superior at 7T, mainly due to higher CNR and more optimal CSF suppression. ${ }^{45}$ However, only approximately $607 \mathrm{~T}$ MRI platforms exist worldwide. Also, the inhomogeneous $B_{0}$ and $B_{1}$ fields can still hamper assessment of peripheral parts of the brain, such as in the Sylvian fissure, although this is improving over time due to the continuous development of the technology. Combined with the fact that 7T MRI has not yet been officially approved for clinical diagnostic imaging, 7T vessel wall MRI sequences are mainly used for research purposes and specific challenging clinical cases (e.g., cerebral vasculitis).

\section{Image contrast weighting}

Mirroring pulse sequence development in the extracranial carotid artery, pulse sequences with different image contrast weighting (e.g., $\mathrm{T}_{1}-, \mathrm{T}_{2}$ - and PD-weighted) have also been developed for assessing the intracranial vessel wall. Theoretically, the ideal vessel wall MRI protocol would include images of all three image contrast weightings and a $T_{1}$-weighted sequence after contrast administration to assess intracranial vessel wall pathology in the same fashion as has been done for years for their extracranial counterparts. ${ }^{46}$ However, no clear evidence yet exists on the clinical relevance of multicontrast MRI protocols for intracranial vessel wall pathology. With the relatively long acquisition times of these vessel wall sequences, most of the currently used intracranial vessel wall MRI protocols rely on $\mathrm{T}_{1}$-weighted imaging, because of its benefits in the detectability of contrast enhancement after contrast administration and favorable imaging characteristics when distinguishing the vessel wall with its surrounding tissue and (if present) plaque components. ${ }^{47}$ 


\section{Our $3 T$ protocol}

The 3T MRI systems at our center (Achieva 3.0T, Philips Healthcare, Best, The Netherlands) were recently equipped with a 32-channel phased array SENSE head coil. Previously, we used an 8-channel head coil. We clinically use a 3D $T_{1}$ weighted Volumetric Isotropically Reconstructed Turbo Spin-Echo Acquisition (VIRTA) sequence (adapted from Qiao et al.) 17,42 that is acquired after contrast administration only (gadobutrol, Gadovist $1.0 \mathrm{mmol} / \mathrm{mL}$, single dose, adjusted to patient weight; Bayer Schering Pharma, Newbury, England). The images are acquired at an anisotropic spatial resolution of $0.5 \times 0.5 \times 1.0 \mathrm{~mm}^{3}$ that is subsequently reconstructed to $0.5 \times 0.5 \times 0.5 \mathrm{~mm}^{3}$ isotropic resolution. We have chosen this particular spatial resolution, which is considerably below the 'ideal' spatial resolution of $0.18 \times 0.18 \times 0.18 \mathrm{~mm}^{3}$ mentioned above, because of the limitations regarding acquisition time and SNR. Also, in a recent small study, we found that sequences with a lower spatial resolution $\left(0.5 \mathrm{~mm} \times 0.5 \mathrm{~mm} \times 1.0 \mathrm{~mm}^{3}\right)$ and a short scan duration (4 minutes 39 seconds) have a good subjective quality score and good performance with respect to lesion detection. ${ }^{39}$ An anisotropic sequence was chosen for two main reasons: (a) In our experience, assessment of the in-plane slices, planned in a transverse oblique plane to image the circle of Willis in the classic anatomical way (Figure 1), most often will suffice for detection of larger lesions; and (b) we mainly use this sequence to detect vessel wall enhancement, which is less dependent on partial volume effects. In this regard, an anisotropic voxel size can in our opinion be a good compromise with an increase in SNR and shorter acquisition times. However, the above-mentioned drawbacks of anisotropic sequences always need to be taken into account when assessing the images.

In the VIRTA sequence, black blood is obtained by means of low flip-angle refocusing pulses. To optimize CSF suppression, we use the anti-driven-equilibrium (ADE) method. 17,33,39 Because CSF suppression is not optimal using this method, we have currently started implementing DANTE for better CSF suppression. Because the FOV of the sequence is restricted in the through-plane direction - measuring $45 \mathrm{~mm}$ thickness - care must be taken to plan the FOV correctly (Figure 1). Key parameters of our 3T protocol are shown in Table 1. 
The Use and Pitfalls of Intracranial Vessel Wall Imaging

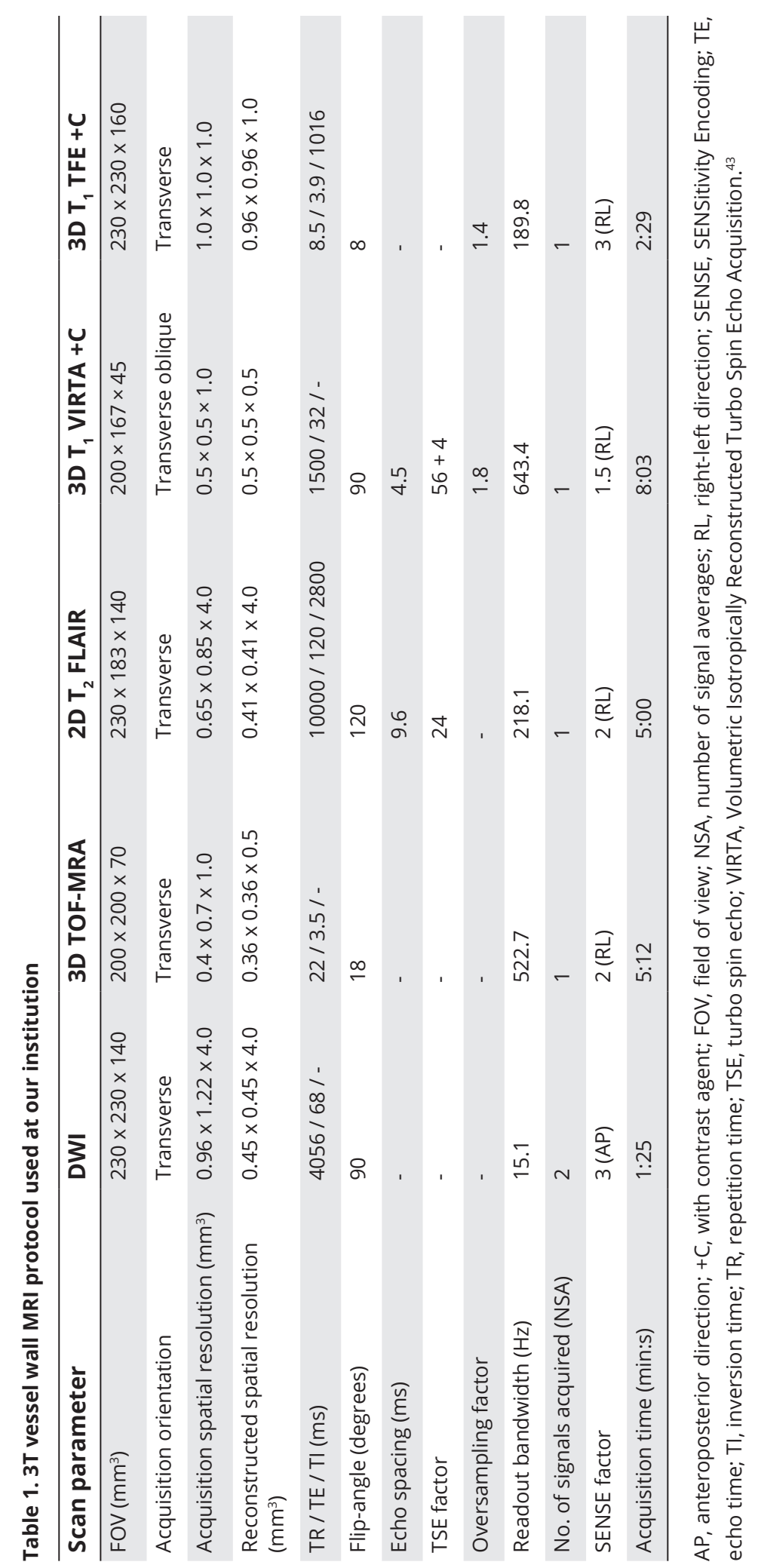


A

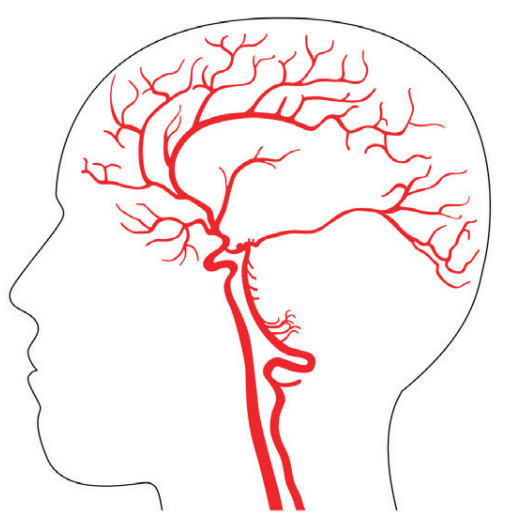

C

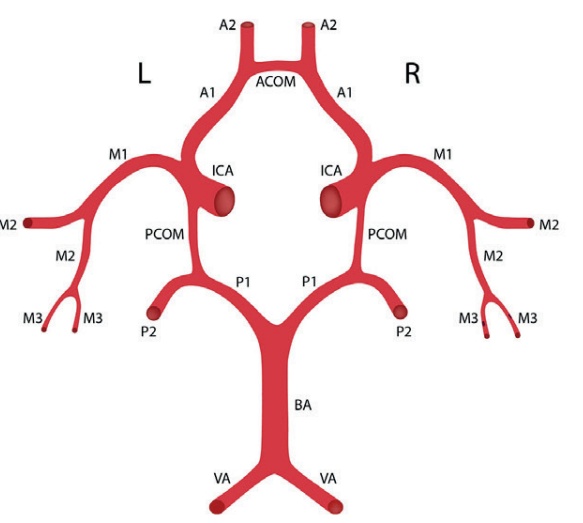

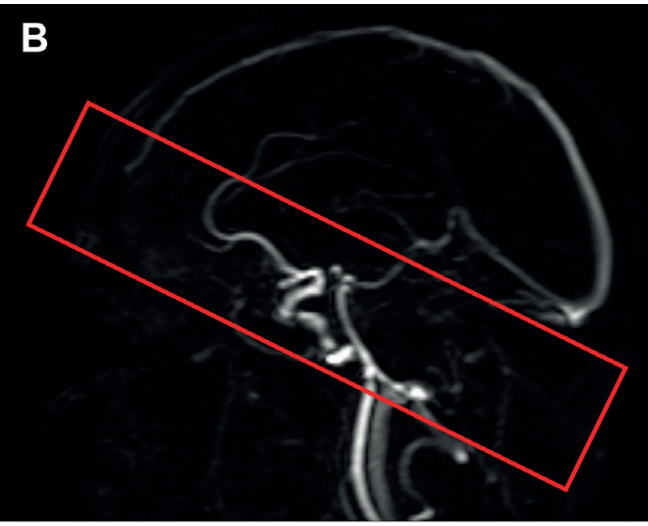

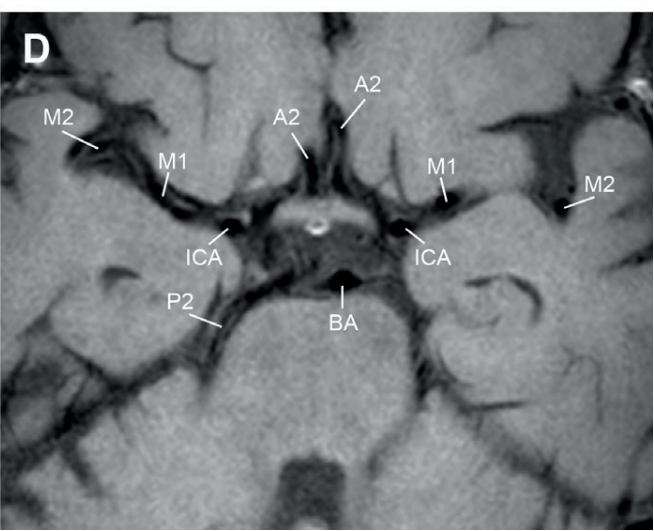

Figure 1. (A) Sagittal view of the anterior circulation intracranial arteries and (B) corresponding phase-contrast angiography image used in our clinic for planning the field of view ( $45 \mathrm{~mm}$ feet-head, red box) in a transverse oblique plane to include all large intracranial arteries of the anterior circulation. (C) Transverse view of the large intracranial arteries of the circle of Willis and (D) corresponding transverse oblique oriented 3T $\mathrm{T}_{1}$-weighted intracranial vessel wall MR image, in which most anterior circulation arteries can be seen in one slice. A1 and $\mathrm{A} 2$, segments of the anterior cerebral artery; ACOM, anterior communicating artery; BA, basilar artery; ICA, internal carotid artery; M1, M2 and M3, segments of the middle cerebral artery; P1 and P2, segments of the posterior cerebral artery; PCOM, posterior communicating artery; $\mathrm{VA}$, vertebral artery.

\section{Our $7 T$ protocol}

The 7T MRI platform in our institution (Philips Healthcare, Cleveland, $\mathrm{OH}$, USA) is equipped with a 32-channel receive coil and a volume transmit/ receive coil for transmission (Nova Medical, Wilmington, MA, USA). We use a $\mathrm{T}_{1}$-weighted Magnetization-Prepared Inversion Recovery Turbo Spin-Echo (MPIR-TSE) intracranial vessel wall sequence acquired before and after contrast administration, ${ }^{15,48}$ with acquired isotropic resolution of $0.8 \times 0.8 \times 0.8 \mathrm{~mm}^{3}$ that is reconstructed to $0.49 \times 0.49 \times 0.49 \mathrm{~mm}^{3}$ isotropic resolution. The pulse sequence includes an inversion recovery pulse that results in nearly complete CSF suppression, and uses low and varying refocusing pulse angles for obtaining black blood. The FOV encompasses the entire brain $(190 \mathrm{~mm})$, which, in combination 
with the isotropic spatial resolution, does not require additional planning in oblique angles. $\mathrm{B}_{0}$-shimming and dielectric pads are additionally used to decrease inhomogeneity of the main magnetic and radiofrequency transmit field, which otherwise can result in considerable signal loss in both temporal lobes (including the M2-M3 trajectory). ${ }^{49}$ Key parameters of our 7T protocol are shown in Table 2.

\section{Use of contrast agents}

The use of contrast material-enhanced MR imaging is considered a necessary component of vessel wall MRI protocols.1,2 Sensitivity for contrast enhancement depends on optimal timing of contrast agent injection. Currently, no head-to-head comparisons between vessel wall MRI sequences at different time points after contrast injection have been performed. For timing of contrast enhancement on standard brain MRI sequences, results from studies in brain tumors are generally used.50 As a general rule, optimal timing of sequence acquisition is between approximately 5-10 minutes after contrast agent injection; contrast enhancement may be weak within the first 5 minutes after injection, and evidence of the added value of a late-phase acquisition ( $>10$ minutes) is lacking. Because many of the current vessel wall MRI sequences have an acquisition duration between 5-7 minutes, the ideal start time would be 5 minutes after contrast injection. At our institution, we either perform one anatomic sequence after contrast agent injection - such as an axial $\mathrm{T}_{2}$-weighted TSE or diffusion-weighted imaging (DWI) sequence or implement a 5-minute break before starting the vessel wall MRI sequence.

The choice of whether or not to obtain both pre- and postcontrast images depends on both the specific setting and clinical question. At 7T, we perform the vessel wall MRI sequence before and after contrast material administration, because we mainly examine patients in a research setting or challenging cases and therefore have time (45-60 minute time slot) to acquire both pre- and postcontrast images in addition to the standard anatomical brain images. With this setup, contrastenhancing lesions can be distinguished from non-enhancing lesions with a high signal intensity by comparing pre- and postcontrast images. However, within the context of fixed, shorter MRI time slots and a maximum acquisition time based on patient comfort, in clinical practice it is often necessary to limit the time for intracranial vessel wall MRI at 3T. An option that reduces total protocol time, and which we currently use in our institution, is to only acquire postcontrast images. In our experience, most vessel wall lesions - even when not enhancing - can be detected on the postcontrast vessel wall MRI sequence and it saves half the acquisition time relative to the combination of a pre- and postcontrast sequence. Still, studies are needed to compare this alternative approach (postcontrast-only) to the classic approach (pre- and postcontrast). One of the major limitations of using a postcontrast vessel wall MRI sequence only is that findings such as intraplaque hemorrhage and intracranial arterial dissections may be missed. ${ }^{51}$ 


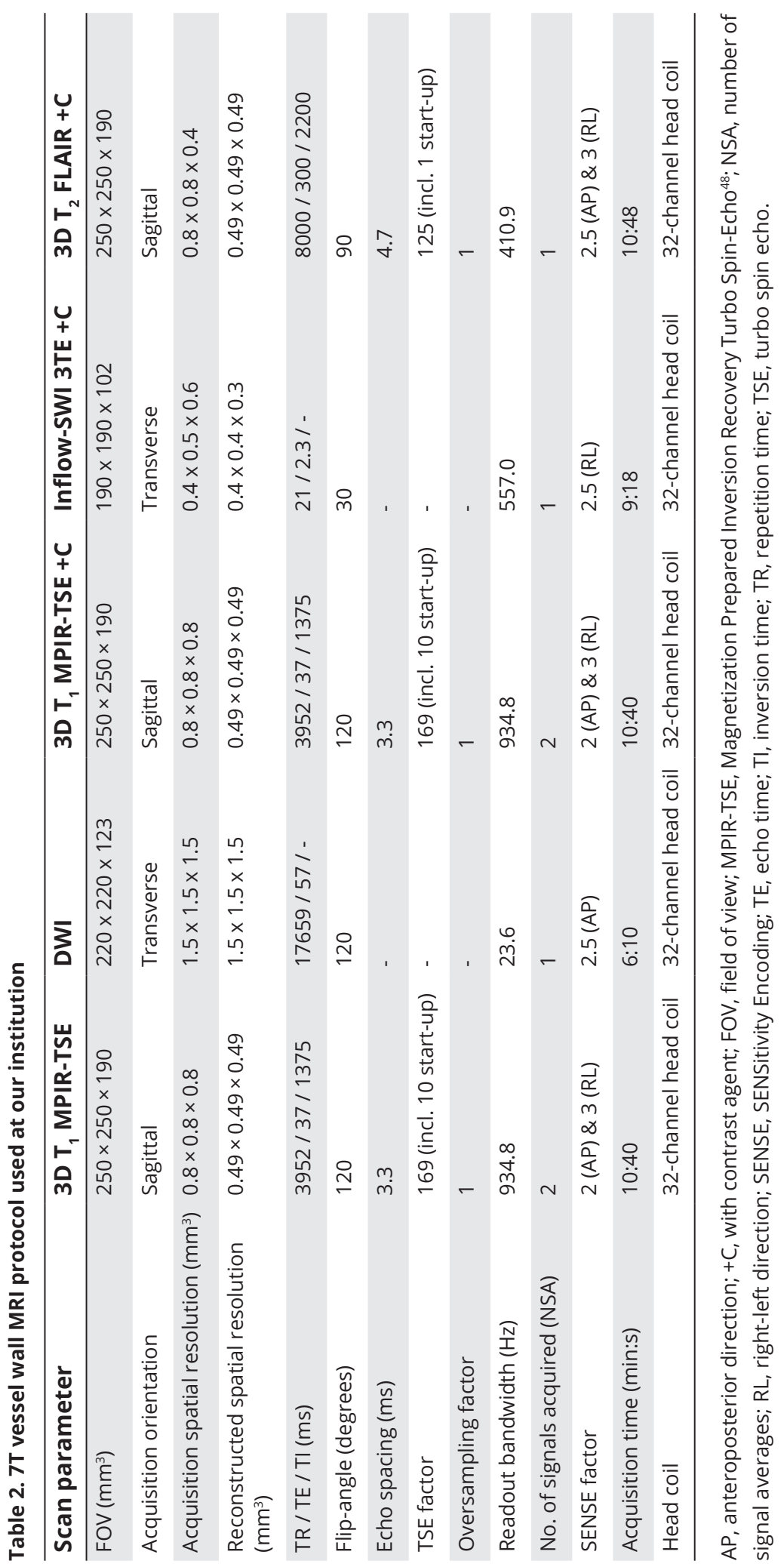




\section{Other necessary sequences}

Other, more 'conventional' MRI sequences also add helpful information when used in combination with intracranial vessel wall MRI. MR angiographic methods, such as contrast-enhanced MRA or time-of-flight (TOF-)MRA are particularly helpful to assess the arterial lumen and to identify the specific arteries to which the visualized vessel walls belong (Figure 2). Also, in case of a small FOV, these techniques can guide FOV planning centered around the circle of Willis or focused on a specific (stenotic) vessel wall lesion. Contrast-enhanced MRA during first contrast passage clearly shows the arterial vasculature and is less sensitive to slow-flow artifacts compared with TOFMRA. Furthermore, with strong elongation of the arterial vasculature it is also less sensitive to a signal decrease of cranial-caudal flow, e.g., in a strongly elongated MCA. Nonetheless, TOF-MRA is still a sensitive method to detect stenotic lesions and is the workhorse in most centers for the detection of vascular disease, including aneurysms of the intracranial vasculature. ${ }^{52}$ Because it is not dependent on first contrast passage, acquisition time can be invested to increase the spatial resolution.

Other pulse sequences that can be of additional value in vessel wall MRI assessment depend on the specific clinical question and include: (a) $\mathrm{T}_{2}$-weighted turbo spinecho (TSE) sequence that can confirm the absence of a flow void in the arterial lumen in a patient with an arterial occlusion, (b) $\mathrm{T}_{1}$-weighted anatomic sequence for both assessment of normal anatomy and for use as 'precontrast' sequence for (mainly tissue) enhancement; (c) $T_{1}$-weighted fat-suppressed sequence that can visualize a subintimal hematoma in patients with an arterial dissection that involves both the extracranial and intracranial segments, and (d) fluid-attenuated inversion recovery (FLAIR)- and DWI sequence for localizing white matter lesions and old and recent ischemia possibly associated with vessel wall pathology. The full vessel wall MRI protocol used in our institution, including key pulse sequence parameters, can be found in Table 1 and Table 2.
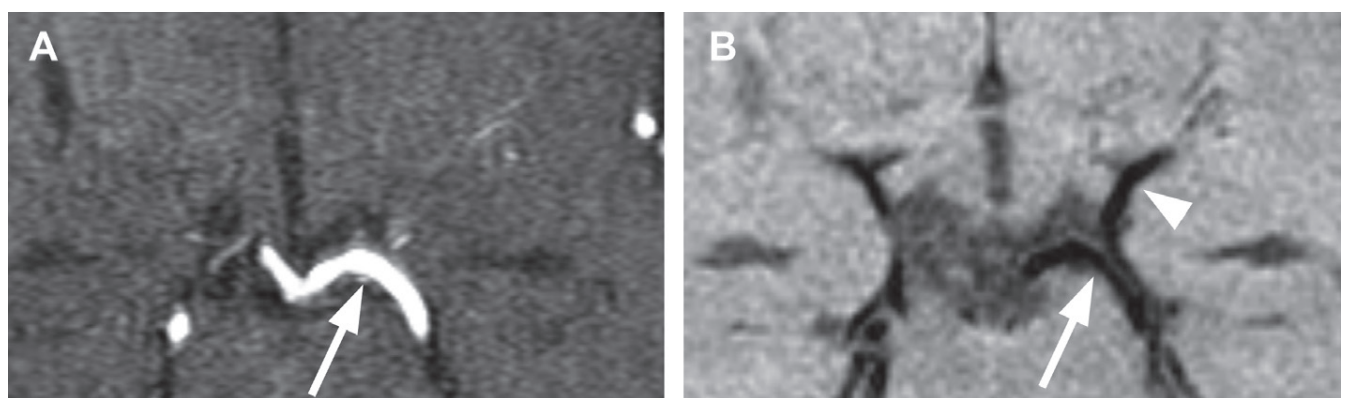

Figure 2. (A) $3 T$ transverse Time-of-Flight (TOF) MR image (voxel size $0.4 \times 0.7 \times 1.0 \mathrm{~mm}^{3}$ ) and (B) corresponding $3 \mathrm{~T}$ transverse oblique precontrast $\mathrm{T}_{1}$-weighted vessel wall image (voxel size $0.5 \times 0.5 \times 1.0$ $\mathrm{mm}^{3}$ ), both zoomed in on the left P1/P2 bifurcation of the posterior cerebral artery (PCA; white arrow). On the vessel wall image (B), the basal vein of Rosenthal can be seen coursing directly lateral to the PCA (arrowhead), which is not visible on the TOF-MRA. 


\section{Patient preparation}

Proper patient preparation plays an important role in the acquisition of high-quality intracranial vessel wall images. The patient (or legal representative) needs to be informed about the MRI examination and the MR imaging staff needs to assess if any possible contraindications for MR imaging (claustrophobia, contraindicated metal objects in or on the body, pregnancy) or for gadolinium-containing contrast agents (known allergic reaction to gadolinium-containing contrast agent, severely impaired renal function) exist. Also, because of the relatively long acquisition time of vessel wall MRI sequences, imaging staff needs to assess whether the patient is clinically able to undergo the examination: it may be challenging to lay still for a long period of time, especially for neurologically impaired patients. Prior to scanning, a peripheral intravenous catheter is placed in the antecubital vein for contrast administration..$^{42,43,53}$

Our complete vessel wall MRI protocol (Table 1 and Table 2 ) takes approximately 23 and 48 minutes for the $3 \mathrm{~T}$ and $7 \mathrm{~T}$ protocol, respectively. The vessel wall MRI sequence has an acquisition time of 8 minutes 3 seconds for the 3T and 10 minutes 40 seconds for the 7T protocol. Vessel wall MRI sequences are susceptible to motion artifacts because of the relatively long acquisition duration, and visibility of the vessel wall decreases rapidly when patients move their head during acquisition. We therefore use foam cushions positioned around the patient's head to aid in limiting movement artifacts.

\section{Pre-assessment case preparation - What do we need to know?}

\section{Clinical information}

For a complete assessment of intracranial vessel wall images several clinical aspects are important to know. In general, when requesting vessel wall MRI, the actual clinical status of the patient (neurological status, ability to lie still for a prolonged period of time) should be reported in order to assess the a priori chance of acquiring images of sufficient diagnostic quality. Next, a specific clinical question is important for defining a patient-tailored vessel wall MRI protocol. For instance, if the clinician is suspicious of disease in the anterior cerebral vasculature, the (small) FOV of the 3T sequence needs to be placed in a more transverse orientation in line with the arteries of the anterior circulation part of the circle of Willis. When the area of interest lies predominantly in the posterior circulation, a more transverse oblique angulation can be used to include a longer trajectory of the basilar and vertebral arteries. On the other hand, when there is suspicion of a dissection or intraplaque hemorrhage, a precontrast vessel wall MRI sequence can be added to a postcontrast-only protocol. Further, previous (MRI) examinations showing arterial disease (e.g., stenoses) on conventional sequences need to be taken into account for both FOV planning and evaluation of progression. 
The patient's treatment status is another important clinical feature for vessel wall MRI assessment. For instance, when a patient with cerebral vasculitis is treated with prednisolone, it is important to know when treatment has commenced relative to the time of the imaging examination: vessel wall enhancement may be lower or have disappeared altogether. Also, in case of an ischemic infarct that has been treated with thrombolysis or intra-arterial treatment, there may be local vessel wall enhancement present at the location of the previous occlusion. ${ }^{54,55}$ Finally, if a patient has undergone an MRI examination including contrast administration less than 12 hours before vessel wall MRI, there may be residual contrast enhancement on the subsequent 'precontrast' vessel wall images.

\section{Common indications}

At our institution, the most common indication for our 3T vessel wall MRI protocol is a suspected cerebral vasculitis (either primary angiitis of the central nervous system or due to other causes). When this suspicion is raised by the clinician, a spectrum of symptoms is often present that can also indicate the presence of other vascular diseases, like small vessel disease or large artery atherosclerosis with unstable plaques. Vessel wall MRI can assist in differentiating between these vascular diseases but may also be helpful during follow-up and evaluation of the received treatment. Other vascular diseases in which vessel wall MRI may be indicated are the detection or evaluation (evolution) of large artery atherosclerosis with or without arterial remodeling, and less frequently moyamoya vasculopathy, dissections and cerebral aneurysms (all mostly for research purposes). When $3 T$ vessel wall MRI does not show any abnormalities, when the clinician suspects subtle vessel wall disease, or when all arteries of the circle of Willis need to be thoroughly assessed and/or screened, our 7T protocol can be considered if this platform is available.

\section{Systematic approach of vessel wall assessment}

\section{General assessment}

Vessel wall MRI assessment at our institution is generally performed on specialized workstations for diagnostic imaging, with a high-resolution monitor and using a PACS (picture archiving and communication system, IDS7, Sectra AB, Linköping, Sweden). When an intracranial arterial stenosis has been found, at TOF-MRA, vessel wall MRI could entail a dedicated characterization of this lesion on vessel wall images. ${ }^{8}$ In practice, this is a less common situation in our patient population, so we perform a complete search of the large intracranial vasculature, starting at the extracranial internal carotid and vertebral artery and then following the course of these arteries into the basilar artery and anterior, middle and posterior cerebral artery branches, including the M1, (very) proximal M2, A1, (very) proximal 
A2, P1 and (very) proximal P2 segments. For all currently published vessel wall MRI sequences, including our own sequences, voxel size exceeds the vessel wall thickness of all arteries, and will result in overestimation of wall thickness. Lesions are visible, though, as they generally have a higher intensity or thicker appearance than the adjacent, apparently normal vessel wall. In our experience, most intracranial vessel wall lesions are detectable when scrutinizing the source data of the vessel wall MRI sequences, that is, when the images are acquired in a transverse (7T) or transverse oblique plane (3T). ${ }^{43}$ However, multiplanar reformatting - when applicable - may increase diagnostic confidence of some lesions, including lesions of the M1 segment of the MCA, which is one of the few circle of Willis vessels visualized solely parallel to its orientation in a transverse slice (Figure 1).

Before using intracranial vessel wall MRI as a diagnostic tool in patients, one should become acquainted with the normal appearance of the arterial wall on vessel wall images (Figure 1 and Figure 3). The normal vessel wall can be seen as a thin line surrounding the vessel lumen and is isointense to the brain parenchyma (white and grey matter have approximately the same signal intensity on vessel wall images because sequence parameters are specifically optimized for the vessel wall). Vessel wall MRI of healthy intracranial vasculature frequently suggests a variance in wall thickness that is most likely not related to vessel wall disease but due to partial volume effects. With normal ageing, the vessel wall has been shown to increase in thickness; however, the question remains whether this small increase in thickness can be visualized with the currently used spatial resolutions. ${ }^{45}$ In younger patients without significant brain atrophy, the MCA is often located directly adjacent to the brain parenchyma, with little or no visible CSF in between. Because its signal intensity is similar to that of brain parenchyma in most sequences, it is often difficult to assess all segments of the MCA vessel wall (including possible lesions) (Figure 3). 

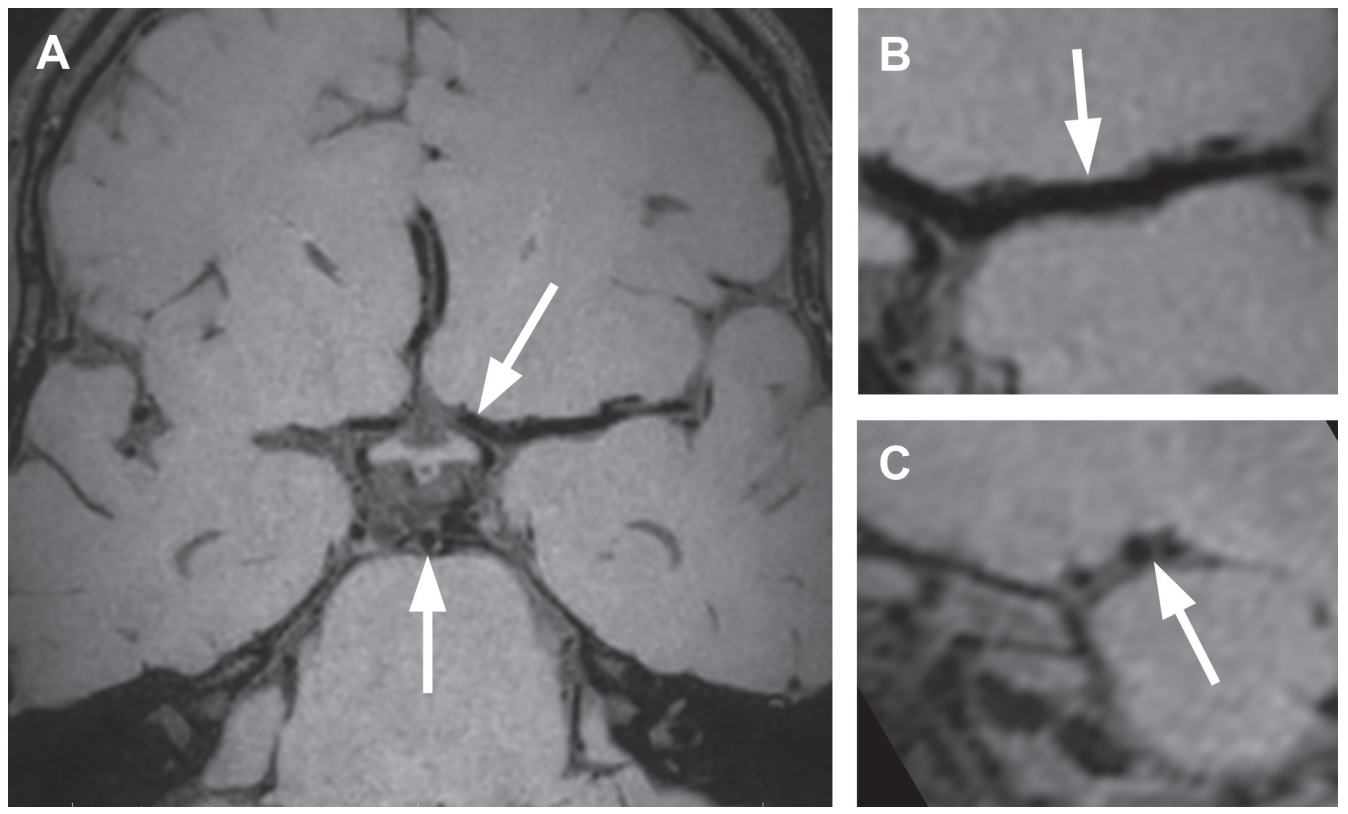

Figure 3. (A) $3 \mathrm{~T}$ oblique (transverse/coronal) precontrast $\mathrm{T}_{1}$-weighted vessel wall image (voxel size $0.5 \mathrm{x}$ $0.5 \times 1.0 \mathrm{~mm}^{3}$ ) of a 29 -year-old healthy volunteer. In this single slice, most large intracranial arteries of the circle of Willis are (partially) visible, e.g., the left A1 segment of the anterior cerebral artery (ACA) and basilar artery (arrows). (B) Zoomed image of the M1 segment of the left middle cerebral artery (MCA). The vessel wall (white arrow) can barely be seen because of its close proximity to the brain parenchyma which has comparable signal intensity. (C) Reconstructed sagittal image $(0.6 \times 1.0$ in-plane spatial resolution) of the left MCA (white arrow). The quality and detail are marginally less compared with the transverse/ oblique image because of the anisotropic voxel size.

In general a vessel wall lesion is defined by one or both of the following characteristics (Figure 4): (a) a focal or more diffuse thickening of the vessel wall greater than $50 \%$ compared with the adjacent vessel wall thickness; ${ }^{31}$ and/or (b) focal or diffuse vivid contrast enhancement. ${ }^{56}$ One can further characterize the lesion as eccentric - less than $50 \%$ of the circumference of the vessel wall - or concentric - greater than $50 \%$ of the circumference - and as enhancing or not enhancing after contrast administration (see below), which can give an indication of the specific underlying disease process (Table 3). When assessing a potential lesion, the area of interest should be compared with next sections (in case of concentric thickening) or the cross-section of the vessel wall should be scrutinized (in case of eccentric thickening), and it should be compared with the contralateral vessel wall (but beware of bilateral disease). Finally, vessel wall images should be cross-correlated with the axial TOFMRA images for correct interpretation of the specific artery/arteries in which the lesion(s) was/were found, and to check if the lesion causes luminal narrowing. Bear in mind that due to arterial remodeling, vessel wall lesions very often do not show a stenosis, ${ }^{15}$ therefore, the absence of stenosis should not be an argument in the decision process whether a lesion is present or not. 

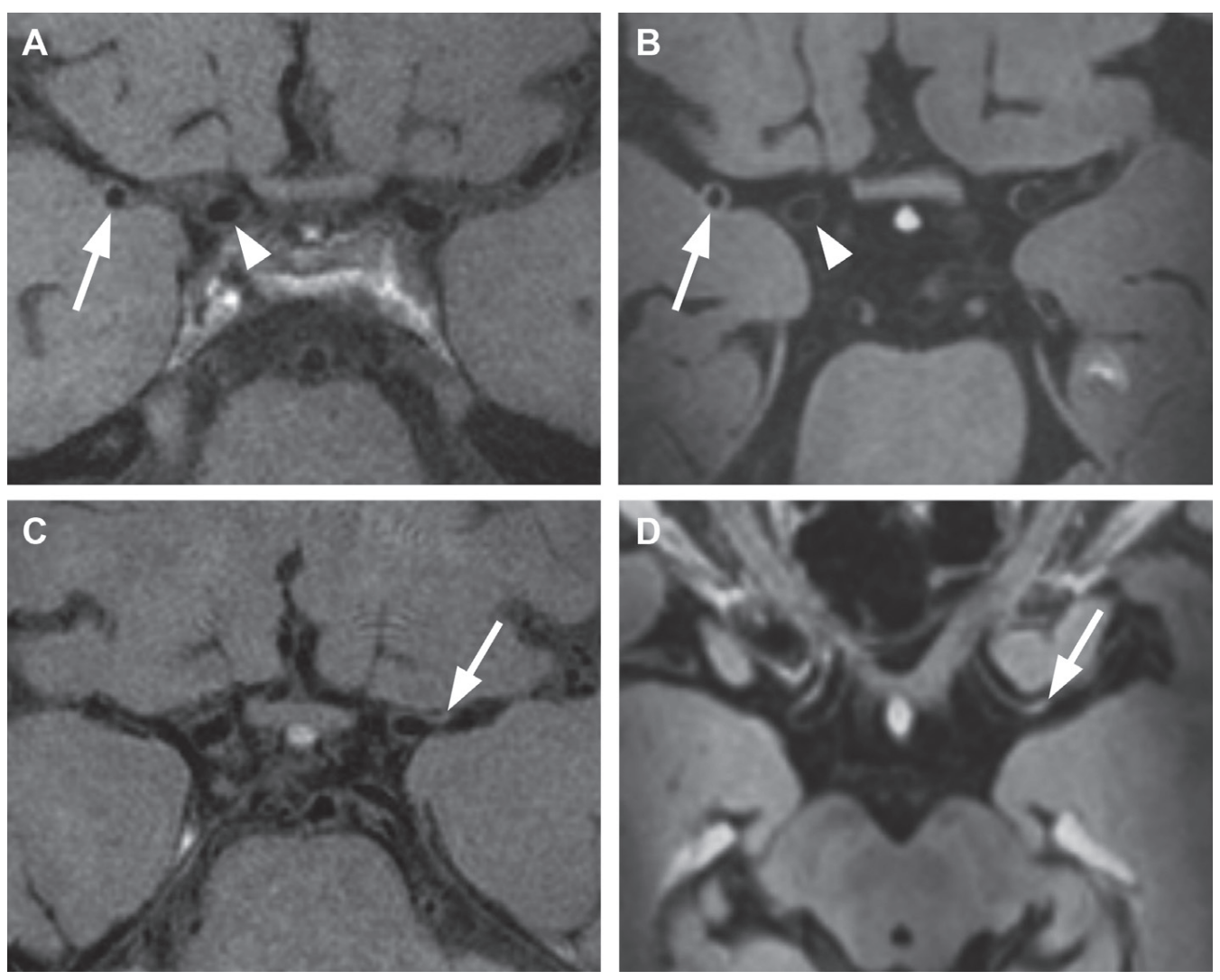

Figure 4. (A and B) 68-year-old female with a recent right-sided ischemic stroke, successfully treated with thrombolytic therapy. Concentric enhancing vessel wall thickening is seen in the right MCA (white arrow) compared with the normal appearing distal ICA (arrowhead) on 3T $\mathrm{T}_{1}$-weighted (A) and 7T $\mathrm{T}_{1}$ weighted MPIR-TSE (Magnetization-Prepared Inversion Recovery Turbo Spin Echo) (B) postcontrast vessel wall images. (C and D) 72-year-old female with a recent transient ischemic attack. Postcontrast 3T (C) and 7T (D) vessel wall images show an eccentric vessel wall lesion (arrow) in the left MCA (just distal to the intracranial internal carotid artery bifurcation). 


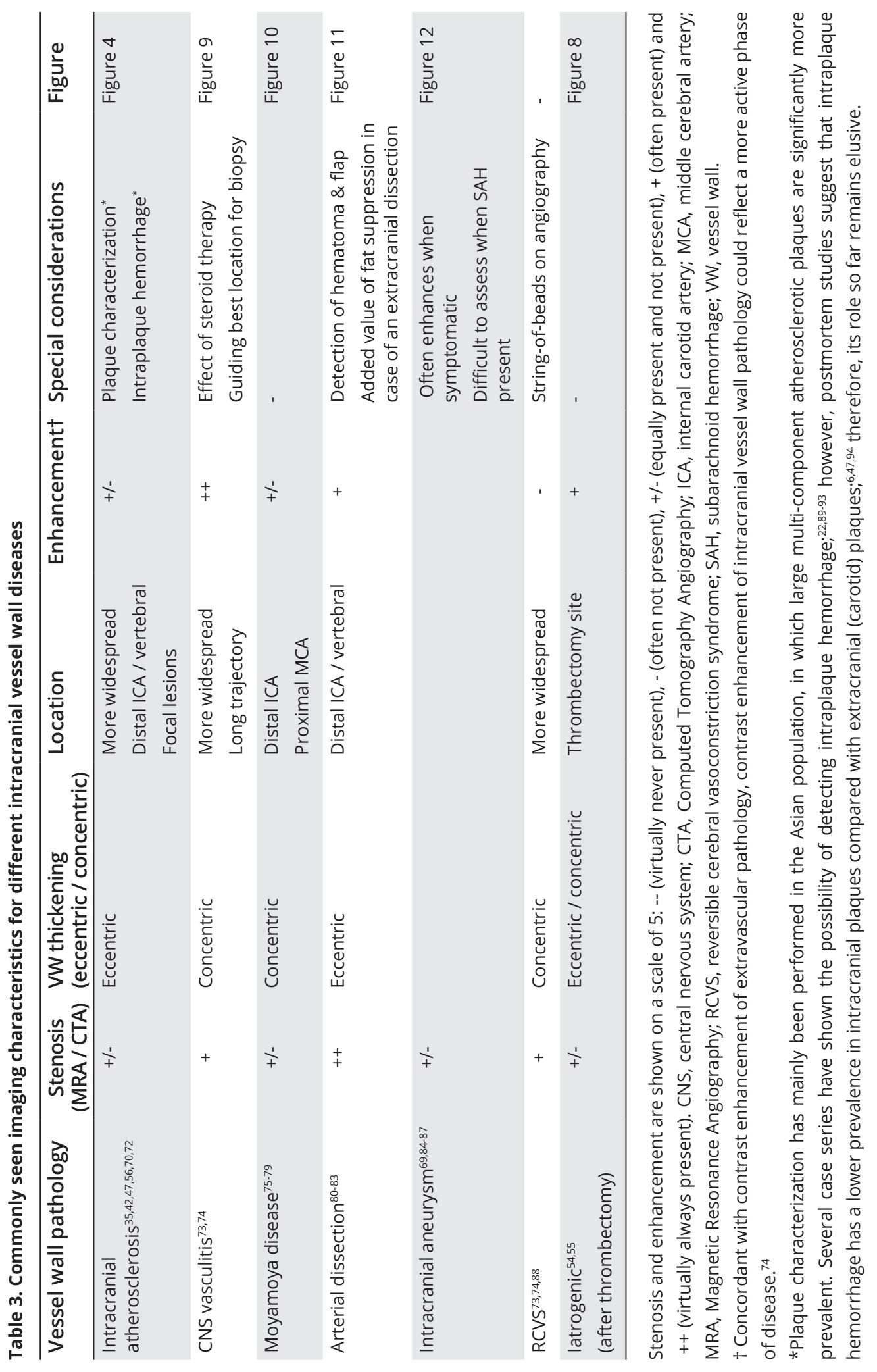




\section{CHAPTER 3}

\section{Contrast enhancement}

Ideally, both pre- and postcontrast images are available for assessment of vessel wall (lesion) enhancement. With this setup contrast-enhancing lesions can be distinguished more accurately from non-enhancing lesions with a high signal intensity. To this end, we either compare pre- and postcontrast images oneon-one (eyeballing), or we calculate subtraction images (postcontrast minus precontrast), for example, using MeVisLab (Version 2.5, MeVis Medical Solutions AG, Bremen, Germany). However, as mentioned before, time constraints often limit vessel wall imaging in clinical practice to one postcontrast vessel wall MRI sequence. When only postcontrast images are available, assessment of contrast enhancement will be based on a comparison of the relative signal intensity of the arterial vessel wall (lesion) with the appearance of the other (contralateral) arterial vessel wall, the signal of the brain tissue and/or the pituitary stalk. The pituitary stalk shows vivid contrast enhancement, ${ }^{57}$ and when the signal intensity of an intracranial arterial vessel wall lesion approximates that of the pituitary stalk, it can be considered as contrast enhancement (Figure 4). Contrarily, if the signal intensity follows the intensity of the brain parenchyma, this can be considered as absence of enhancement.

In healthy subjects, contrast enhancement of the internal carotid artery (ICA) and vertebral artery (VA) wall can be seen at the location where these arteries cross the dura mater, and should not be mistaken for pathological contrast enhancement (Figure 5). Vasa vasorum and increased permeability of the endothelium have been described at this location, but the exact nature of this contrast enhancement has yet to be determined. ${ }^{58}$ Also, the cavernous sinus shows diffuse enhancement after contrast administration; since the ICA runs through this cavernous venous plexus, it is difficult to assess the presence or absence of contrast enhancement of this cavernous segment of the internal carotid artery (Figure 5). 

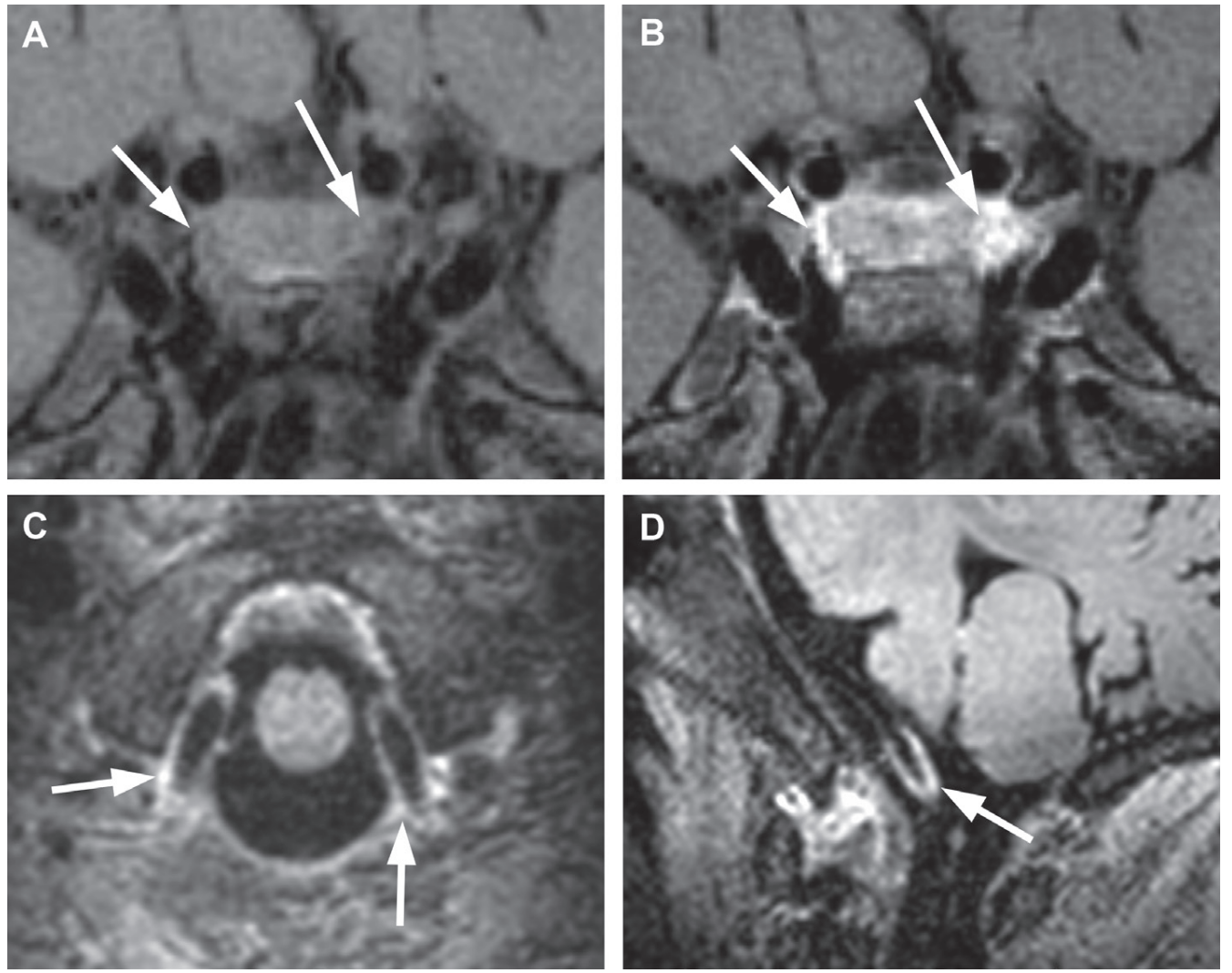

Figure 5. (A and B) 3T pre- (A) and postcontrast (B) $\mathrm{T}_{1}$-weighted vessel wall images (voxel size $0.5 \times 0.5 \times 1.0$ $\mathrm{mm}^{3}$ ) of a 40-year-old female with a recent right-sided ischemic stroke, zoomed in on the cavernous sinus. The postcontrast image of the cavernous sinus shows diffuse contrast enhancement of the cavernous plexus (white arrows) which makes delineation of the carotid vessel wall challenging. (C and $\mathbf{D}) 7 \mathrm{~T}$ postcontrast $\mathrm{T}_{1}$ weighted MPIR-TSE images of the same subject, zoomed in on the vertebral arteries that cross the dura mater. In transverse orientation (C), contrast enhancement (white arrows) of both vertebral arteries can be seen when crossing the dura mater. In sagittal orientation (D) this enhancement can be better appreciated (white arrow) at the same location of the transdural crossing of the vertebral arteries.

\section{Common artifacts \& corresponding pitfalls}

Like all MRI examinations, vessel wall MRI is sensitive to motion artifacts, even more so because of the relatively long acquisition times. Also, SENSE (SENSitivity Encoding) fold-over artifacts need to be considered when deciding to use a vessel wall MRI sequence with SENSE acceleration. Next to these more general artifacts, there are two artifacts that may pose an interpretation problem specifically when assessing vessel wall MRI images. The first is the slow-flow artifact (Figure 6). Due to the parabolic flow velocity profile within a vessel, the flow directly next to the vessel wall is slower compared with the center of the lumen. Intracranial vessel wall MRI sequences that use the inflow or outflow of blood to suppress the arterial lumen can therefore show a higher signal from unsuppressed blood 
near the vessel wall, which mimics either the vessel wall itself (making it appear thickened) or mimics a focal vessel wall lesion (when flow is focally decreased). These hyperintense artifacts can be present both before and after contrast administration but are in principle more visible after contrast injection (Figure 6).
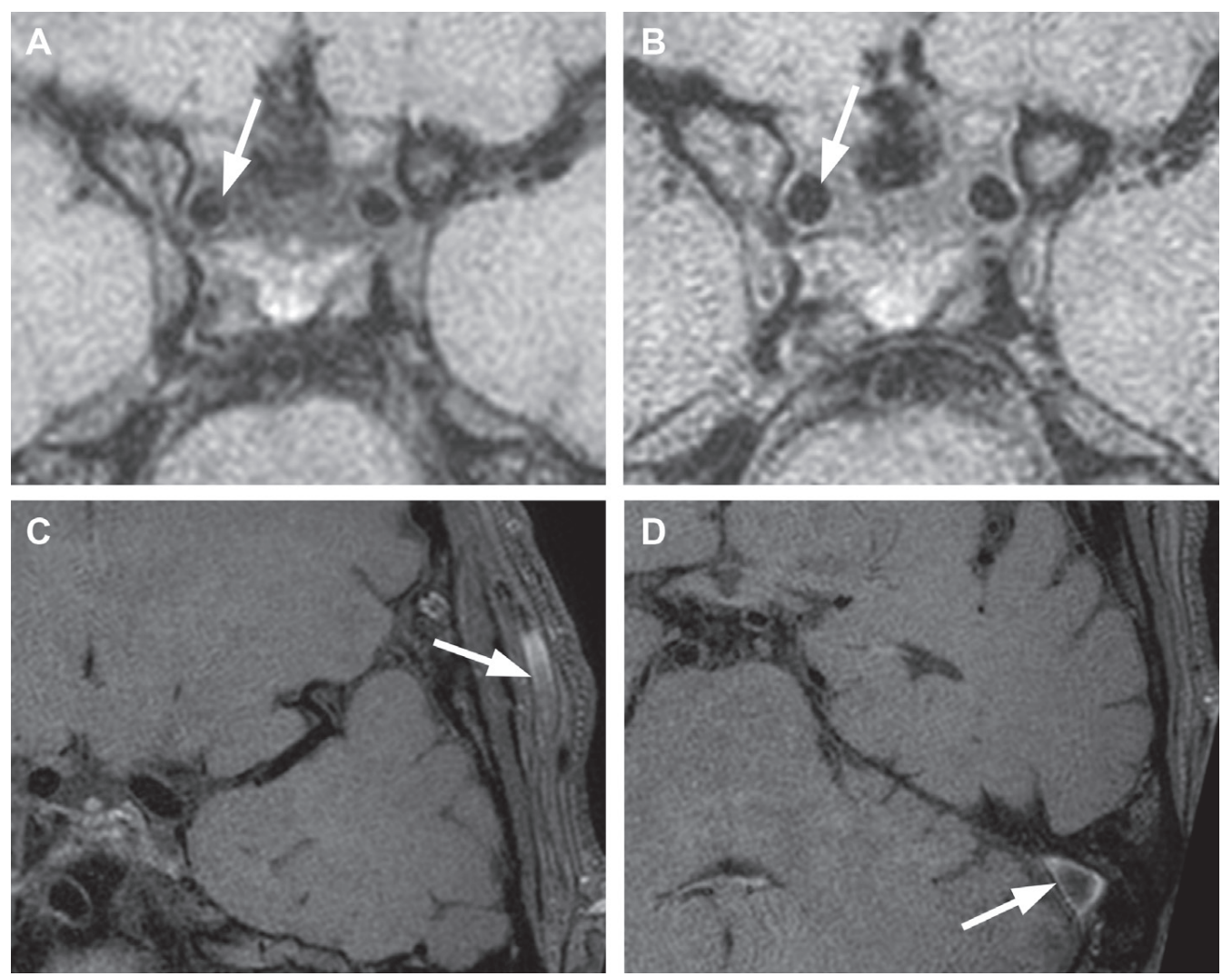

Figure 6. (A and B) 3T precontrast $T_{1}$-weighted vessel wall images (voxel size $0.5 \times 0.5 \times 1.0 \mathrm{~mm}^{3}$ ) of a 38-year-old healthy male. (A) An isointense artifact is seen in the lumen of the right ICA (white arrow). (B) A repeated vessel wall sequence within the same scan session did not show the isointense artifact in the lumen (white arrow). (C and D) $3 \mathrm{~T}$ transverse/oblique postcontrast $\mathrm{T}_{1}$-weighted vessel wall images of a 58-year-old healthy male. Hyperintense venous slow flow artifacts are seen in the extracranial veins (white arrow in C). Hyperintensity caused by slow flow is present in the complete vein, but more prominent along the vessel walls. Slow flow-related hyperintensity can also be observed in the left sigmoid sinus (white arrow in D).

Slow-flow artifacts are most obvious in the slow-flowing blood in smaller and larger veins, for instance in veins within the Sylvian cistern that are in relatively close proximity to the branches of the MCA. When solely interpreting vessel wall images, slow flow in veins can be misinterpreted as arterial wall enhancement. This misinterpretation can be avoided by routinely cross-checking of the location of the intracranial arteries on TOF-MRA images, or by assessing the pattern of 
the hyperintense signal: Slow-flow artifacts are often symmetrically present in both hemispheres and can be seen in many vascular structures both inside the skull and in the extracranial veins, such as the superficial temporal vein (Figure 6). Suboptimal suppression of slow flow is related to the vessel wall MRI technique used, and although TOF-MRA is also prone to slow-flow artifacts, it rarely occurs that these artifacts occur at the exact same location at both sequences. When in doubt about whether a vessel wall abnormality is a slow-flow artifact or a real vessel wall lesion, we do not want to judge every abnormality as a vessel wall lesion, but we analyze every possible lesion with caution; when in doubt, we may not call it a vessel wall lesion; when the hyperintense signal is too large in volume for a thin arterial vessel wall, we consider it a slow-flow artifact.

The other artifact that can cause difficulties in interpretation of vessel wall abnormalities is the free induction decay (FID) artifact (Figure 7). This artifact is caused by repeated refocusing radiofrequency pulses within the short echo times of $\mathrm{T}_{1}$-weigthed vessel wall MRI sequences. The artifact occurs when the free induction decays from the refocusing pulses are not completely spoiled, and appears as a linear dashed pattern in the image. ${ }^{59}$ The vessel wall will show the same dashed, 'zigzag' pattern, making it difficult to differentiate between artifact and vessel wall thickening and/or lesion. Possible ways to minimize this artifact is to increase the number of signals acquired, increase the echo time or choose a larger slice thickness; however, for obvious reasons these are difficult to implement in intracranial vessel wall MRI.

\section{Vessel wall disease}

Although intracranial vessel wall MRI is still in its developmental stage, there are already several challenging diagnostic situations in which it has shown its potential value, ${ }^{1,2,60,61}$ the most important of which is determining the cause of stroke and assessing accompanying vessel wall lesions. Recently, two excellent review papers have been published that both address the commonly found imaging characteristics of different types of intracranial vessel wall pathology, including a thorough literature overview. ${ }^{2,62}$ We will therefore restrict this section to a compact overview table (Table 3) for hands-on use in clinical practice, including illustrative image examples (Figure 4, Figure 8, Figure 9, Figure 10, Figure 11 and Figure 12). 


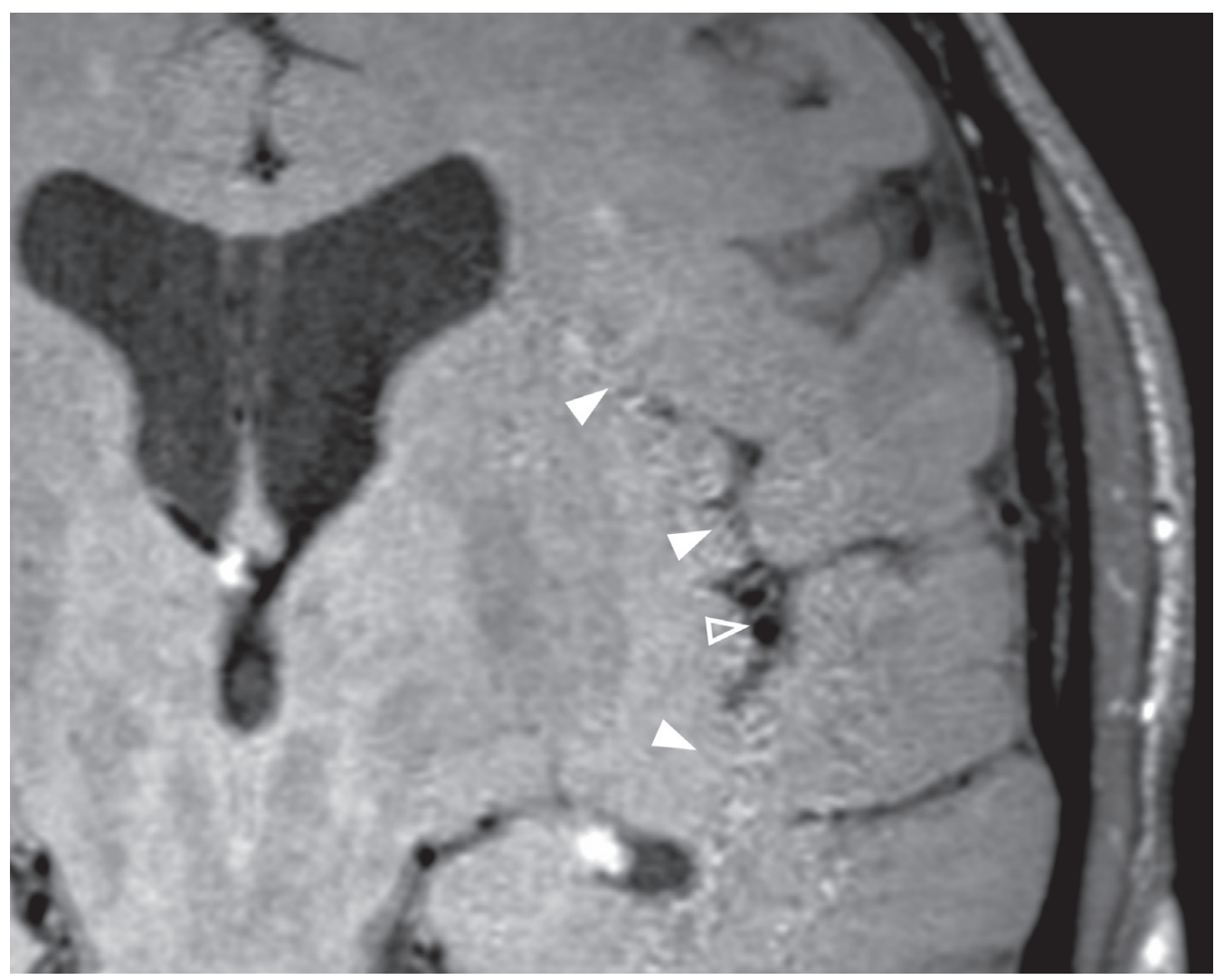

Figure 7. An example of a free induction decay (FID) artifact in a $3 T$ precontrast $\mathrm{T}_{1}$-weighted vessel wall image (voxel size $0.5 \times 0.5 \times 1.0 \mathrm{~mm}^{3}$ ). A dashed pattern is seen over the left hemisphere (white arrowheads). When the artifact is present at the same location of a vessel wall (open arrowhead) it hampers interpretation of vessel wall abnormalities.
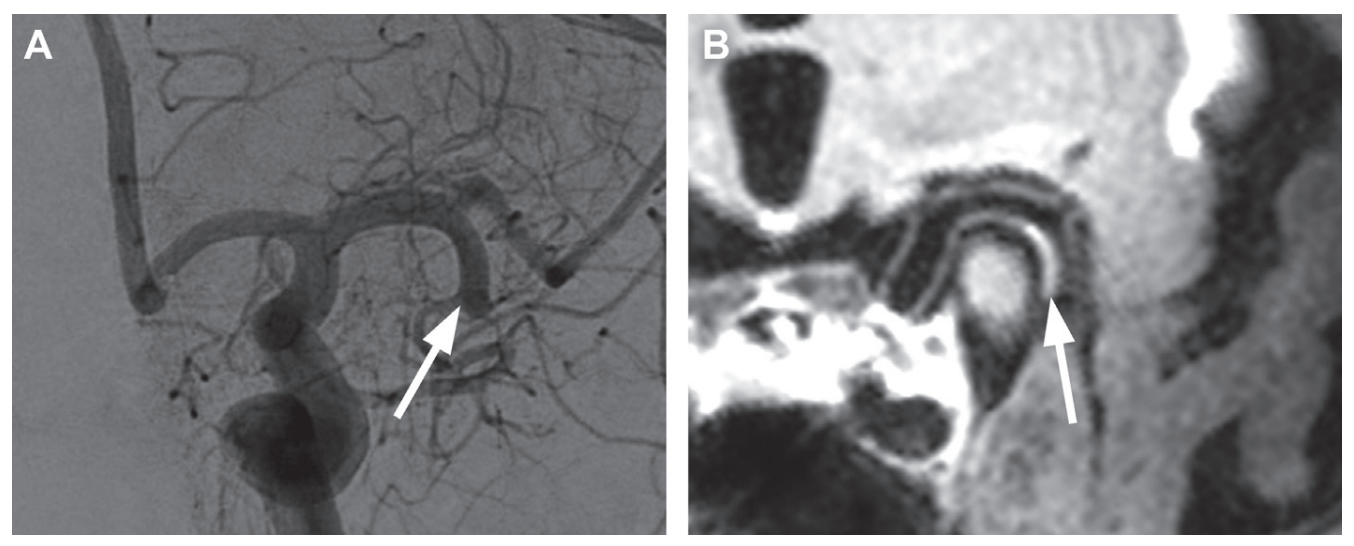

Figure 8. 85-year-old male with a recent occlusion of the left MCA, successfully treated with mechanical thrombectomy. (A) Intra-arterial Digital Subtraction Angiography (DSA) directly before the mechanical thrombectomy procedure, showing an acute cut-off in the MCA (white arrow). (B) On the 7T T $\mathrm{T}_{1}$-weighted MPIR-TSE image, clear vessel wall enhancement in the MCA can be seen at the location of the M1-M2 segment (white arrow) where the thrombectomy device removed the occluding thrombus. 

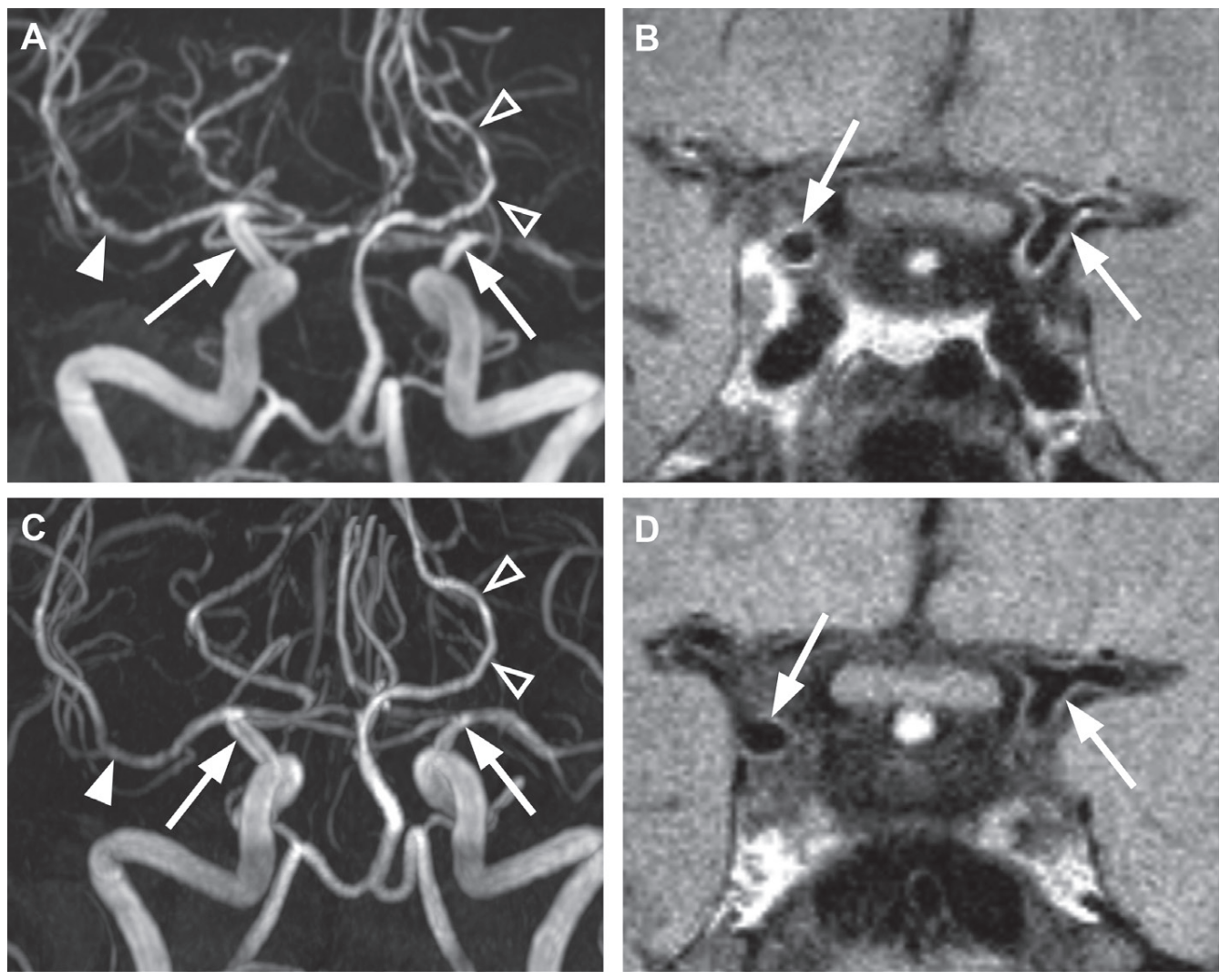

Figure 9. Vessel wall MRI in a 47-year-old male with a clinically proven primary angiitis of the Central Nervous System (PACNS). Although biopsy was inconclusive, DSA showed extensive central and peripheral segmental arterial narrowing. (A and C) 3T coronal maximum intensity projections (maxIPs) of the 3D TOF-MRA and (B and D) 3T transverse postcontrast $T_{1}$-weighted vessel wall images (voxel size $0.5 \times 0.5 \times 1.0 \mathrm{~mm}^{3}$ ) at presentation (A and $\mathbf{B}$ ) and 7 months after immunosuppressive therapy (C and D). At presentation, the TOF-MRA shows multi-segmental luminal narrowing in PCA and MCA (respective open and white arrowheads in (A)) and left distal ICA (right arrow), while the corresponding vessel wall images (voxel size $0.5 \times 0.5 \times 1.0 \mathrm{~mm}^{3}$ ) (B) show more extensive disease, with clear concentric vessel wall enhancement of the left distal ICA-A1-M1 bifurcation (right arrow) as well as the right distal ICA (left arrow). After therapy, a reduction in the multi-segmental luminal narrowing can be seen on the TOF-MRA (respective open and white arrowheads in $(\mathbf{C})$ ), while $\mathrm{T}_{1}$-weighted vessel wall MR images shows resolution of vessel wall enhancement in both distal ICAs (white arrows). 

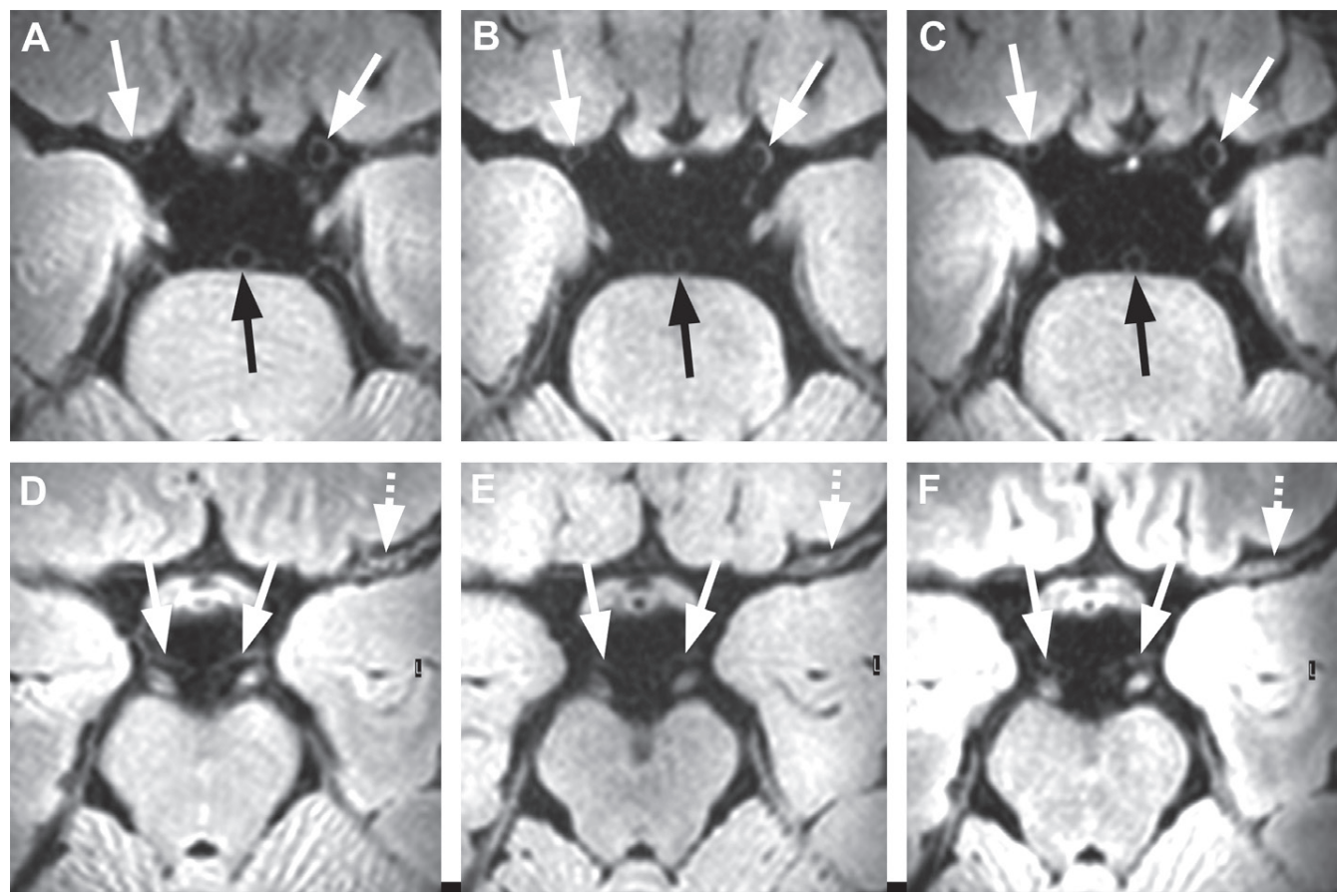

Figure 10. 7T transverse $T_{1}$-weighted MPIR-TSE (A and $\mathbf{D}$ ), whole brain $T_{1}$-weighted (B and $\mathbf{E}$ ) and $T_{2}$ weighted MPIR-TSE images ( $\mathbf{C}$ and $\mathbf{F}$ ) of a 31-year-old man with moyamoya disease. The vessel wall of the left distal ICA (left white arrow in A-C) is slightly more hyperintense compared with the right distal ICA (right white arrow in A-C) and basilar artery (black arrow in A-C). Both distal ICAs are relatively narrow compared with the basilar artery. (D-F) The left MCA is seen to be almost occluded with vessel wall thickening (dashed arrow). The vessel walls of the P1 segment of the PCA are also visible (white arrows). Figure reprinted with permission from: AG van der Kolk. ${ }^{48}$ 

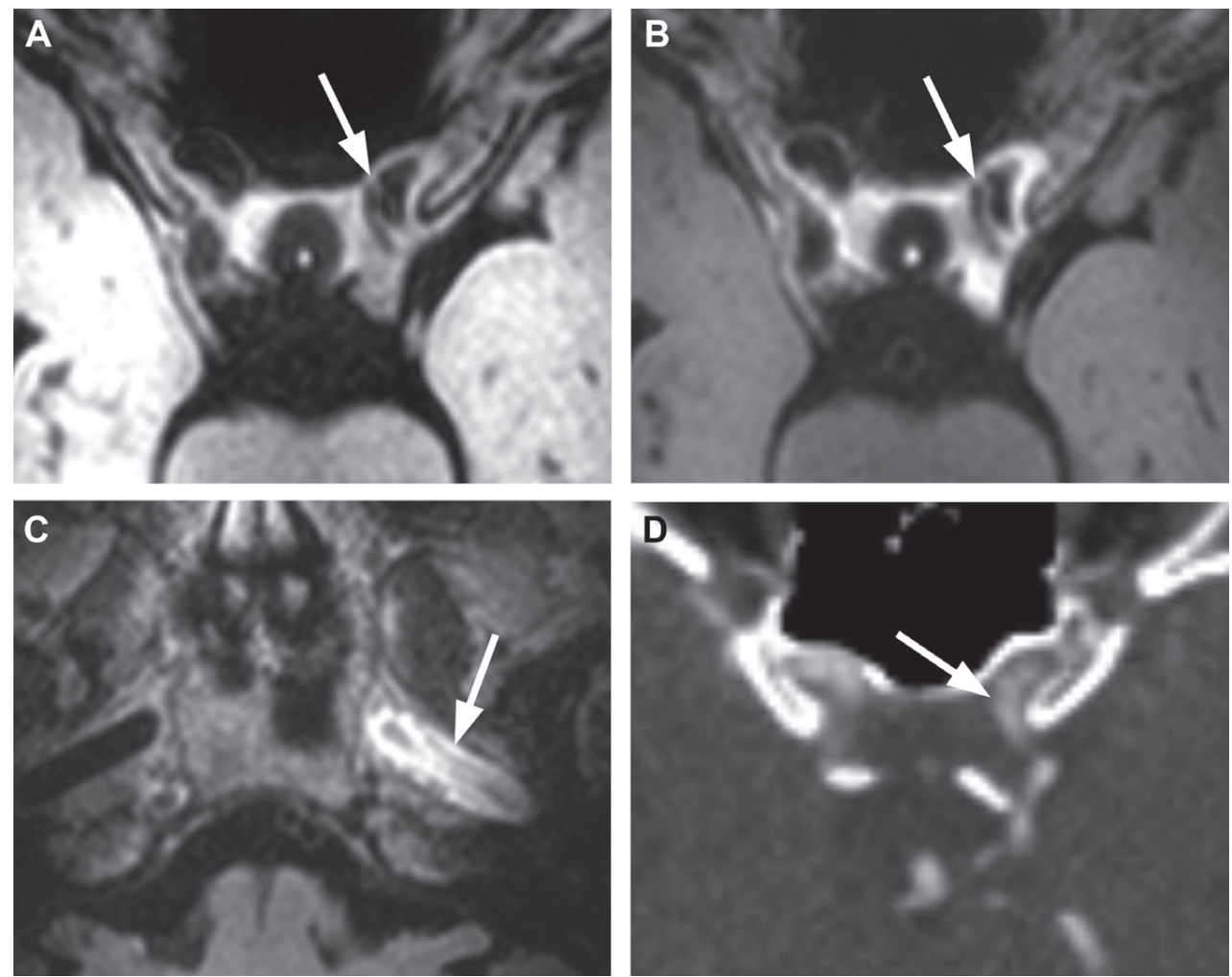

Figure 11. (A-C) 7T transverse vessel wall MRI of a 44-year-old female with a spontaneous dissection of the left ICA, scanned 4 days after symptom onset. (A and B) 7 T transverse precontrast (A) and postcontrast (B) $\mathrm{T}_{1}$-weighted MPIR-TSE images show a tapering of the lumen and vessel wall enhancement (white arrow) of the left distal ICA. (C) Diffuse concentric vessel wall enhancement and a dissection flap are seen on the postcontrast images more proximally in the left distal ICA at the skull base (white arrow). (D) Tapering of the distal ICA can also be appreciated on CTA (Computed Tomography Angiography), reformatted in the same orientation as (A) and (B).
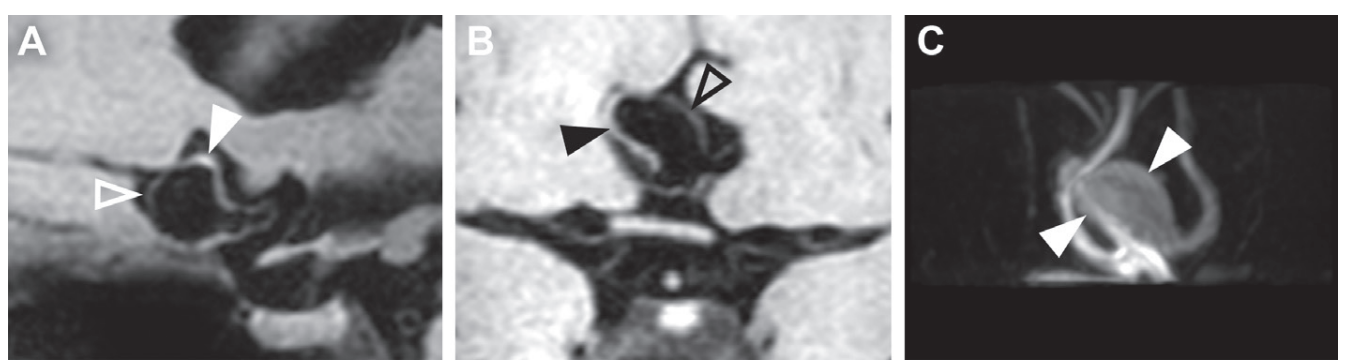

Figure 12. 7T precontrast sagittal (A) and coronal (B) $T_{1}$-weighted MPIR-TSE images of an anterior communicating artery (ACOM) aneurysm. The aneurysm wall shows a variation in wall thickness: the left lateral side and top of the aneurysm can be seen to have a thicker wall (white and black arrowhead), while the right lateral and anterior part of the aneurysm show a thinner wall (white and black open arrowhead). (C) 7T maximum intensity projection of the 3D TOF-MRA shows the corresponding anatomical geometry of the aneurysm (arrowheads). 


\section{Future prospects}

\section{Technical developments}

As discussed in the previous paragraphs, the difficulty in intracranial vessel wall MRI is that ideally, one strives for a maximum spatial resolution to detect lesions of the small intracranial arterial vessel walls, and maximum suppression of both blood signal within and CSF signal surrounding the arteries. Both will significantly increase acquisition time. For clinical implementation, acquisition time reduction is necessary to perform both pre- and postcontrast vessel wall MRI within a clinical MRI time slot (approximately 25-35 minutes), without a substantial decrease in SNR and CNR of the arterial vessel wall. Innovative methods for reducing acquisition time (e.g., compressed sensing ${ }^{63,64}$ ), as well as different CSF suppression techniques (DANTE, ${ }^{19,20,65}$ ADE ${ }^{32,33}$ ), in combination with various 2D- and 3D acquisition methods with different coverage, need to be compared in the upcoming years.

\section{Spatial coverage}

At 3T, most sequences are limited to a region that covers either the circle of Willis or the (known) stenotic vessel wall lesion, with a few surrounding centimeters. An option for 'increasing coverage' is to acquire the vessel wall images in a more angulated coronal plane and include the proximal vertebral arteries, as we generally do with our 3T sequence. 'Real' whole-brain coverage - i.e. increasing the FOV - benefits from non-selective pulses (shorter echo spacing in TSE trains), 2D SENSE and no need for oversampling. ${ }^{48}$ However, because flow suppression is often based on dephasing during the echo train, with too short echo train lengths flow suppression will inevitably decrease.

Next to improvements in pulse sequence design, hardware improvements may also show promise in vessel wall MRI. Recently, an advanced coil system for joint intracranial and extracranial vessel wall imaging has been developed. ${ }^{66}$ This coil system provides the opportunity to image both intracranial and extracranial arteries at once for an optimal assessment of the association of carotid and intracranial atherosclerotic plaques and ischemic stroke within a reasonable scan time (5 minutes 54 seconds to 7 minutes 36 seconds).

\section{Acquisition time}

Efficient $k$-space sampling trajectories (view-ordering) in combination with parallel imaging techniques have been described to reduce acquisition time in 3D turbo spin-echo sequences, while the trajectories can also be optimized for reduced $\mathrm{T}_{2}$-weighting. ${ }^{17,23}$ Compressed sensing allows image reconstruction from fewer $k$-space data and, thus, shorter scan times. Compressed sensing needs yet to be investigated for intracranial vessel wall MRI but might be a way to further reduce the acquisition time. ${ }^{63,64}$ 
In current protocols, several scan parameters can be varied to counterbalance the relatively long acquisition time, such as the oversample factor, TSE train length, and image acceleration by parallel imaging such as SENSE that leads to a change in imaging time. ${ }^{1,21,31,40}$ However, all of these techniques sacrifice spatial resolution and/or SNR in the process, or need more advanced hardware (e.g., a higher number of receiver coils or multi-band scanning) to overcome the inevitable cost in image quality.

\section{Clinical considerations}

Because no gold standard in vivo method for intracranial arterial vessel wall pathology is available, histologic validation of intracranial vessel wall MRI is essential. Since no tissue can be obtained while the patient is alive - compared with, for example, endarterectomy samples in carotid artery disease - validation can only be performed in postmortem studies. A series of postmortem validation studies has been performed at 7T by using ex vivo circle of Willis specimens from patients with and without a history of cerebrovascular disease, as well as from patients with intracranial aneurysms. ${ }^{47,67-71}$ These studies found clear correlations between vessel wall and atherosclerotic plaques detected on vessel wall MRI and histopathological findings, best seen on the $T_{1}$-weighted sequences. ${ }^{47,67,68,70,71}$ However, more insight into what normal ageing of the vessel wall looks like on vessel wall MRI, and what underlying mechanisms can cause vessel wall (lesion) enhancement are still needed. This has proven to be a challenge, because methods to preserve tissue in ex vivo studies (e.g., fixation and tissue temperature effects) change the MRI characteristics of the tissue so that results from these studies cannot be directly translated to in vivo vessel wall MRI, while functional measures, such as lesion enhancement after contrast administration, cannot be performed in postmortem studies.

\section{Conclusion}

Intracranial vessel wall MRI has become part of state-of-the-art MRI protocols detecting causes of ischemic stroke, mainly in a research setting but increasingly asked for (and used) in clinical practice. It has tentatively shown commonly seen vessel wall changes in patients with diseases including, but not limited to, central nervous system vasculitis, moyamoya disease, aneurysms, dissections and intracranial atherosclerosis. However, its precise role and added value for prognosis and patient care needs further elucidation. A field strength of at least 3T enables vessel wall MRI sequences with high enough CNR and spatial resolution to assess the thin intracranial atherosclerotic vessel wall and detect and ultimately characterize vessel wall lesions. Radiologists should be aware of the normal appearance (variance) of the vessel wall on intracranial vessel wall MRI, the main characteristics of vessel wall lesions that 
may help differentiate between different vessel wall pathologic conditions, and the technical limitations and pitfalls in the assessment of intracranial vessel wall MRI. Further technical improvements will enable (among others) reduced acquisition time, while further histological validation of these vessel wall MRI sequences will aid in a better understanding of normal vessel wall thickness variance, ultimately leading to better knowledge of the underlying pathologic conditions of lesions seen on intracranial vessel wall MRI. 


\section{References}

1. Dieleman N, van der Kolk AG, Zwanenburg J, et al. Imaging intracranial vessel wall pathology with magnetic resonance imaging: current prospects and future directions. Circulation 2014;130:192-201.

2. Mandell DM, Mossa-Basha M, Qiao Y, et al. Intracranial Vessel Wall MRI: Principles and Expert Consensus Recommendations of the American Society of Neuroradiology. AJNR American journal of neuroradiology 2017;38:218-229.

3. Gorelick PB, Wong KS, Bae HJ, Pandey DK. Large artery intracranial occlusive disease: a large worldwide burden but a relatively neglected frontier. Stroke 2008;39:2396-9.

4. Arenillas JF. Intracranial atherosclerosis: current concepts. Stroke 2011;42:S20-3.

5. Qureshi Al, Caplan LR. Intracranial atherosclerosis. Lancet 2014;383:984-98.

6. Ritz K, Denswil NP, Stam OC, van Lieshout JJ, Daemen MJ. Cause and mechanisms of intracranial atherosclerosis. Circulation 2014;130:1407-14.

7. Chimowitz MI, Lynn MJ, Howlett-Smith $\mathrm{H}$, et al. Comparison of warfarin and aspirin for symptomatic intracranial arterial stenosis. The New England Journal of Medicine 2005;352:1305-16.

8. Holmstedt CA, Turan TN, Chimowitz MI. Atherosclerotic intracranial arterial stenosis: risk factors, diagnosis, and treatment. The Lancet Neurology 2013;12:1106-14.

9. Derdeyn CP, Chimowitz MI, Lynn MJ, et al. Aggressive medical treatment with or without stenting in high-risk patients with intracranial artery stenosis (SAMMPRIS): the final results of a randomised trial. Lancet 2014;383:333-41.

10. Bos D, Portegies ML, van der Lugt A, et al. Intracranial carotid artery atherosclerosis and the risk of stroke in whites: the Rotterdam Study. JAMA Neurology 2014;71:405-11.

11. Bos D, van der Rijk MJ, Geeraedts TE, et al. Intracranial carotid artery atherosclerosis: prevalence and risk factors in the general population. Stroke 2012;43:1878-84.

12. Homburg PJ, Plas GJ, Rozie S, van der Lugt A, Dippel DW. Prevalence and calcification of intracranial arterial stenotic lesions as assessed with multidetector computed tomography angiography. Stroke 2011;42:1244-50.

13. Vos A, Van Hecke W, Spliet WG, et al. Predominance of Nonatherosclerotic Internal Elastic Lamina Calcification in the Intracranial Internal Carotid Artery. Stroke 2016;47:221-3.

14. Nguyen-Huynh MN, Wintermark M, English J, et al. How accurate is CT angiography in evaluating intracranial atherosclerotic disease? Stroke 2008;39:1184-8.

15. Van der Kolk AG, Zwanenburg JJ, Brundel M, et al. Intracranial vessel wall imaging at 7.0T MRI. Stroke 2011:42:2478-84.

16. Van der Kolk AG, Hendrikse J, Zwanenburg JJ, Visser F, Luijten PR. Clinical applications of 7 T MRI in the brain. European Journal of Radiology 2013;82:708-18.

17. Qiao Y, Steinman DA, Qin Q, et al. Intracranial arterial wall imaging using threedimensional high isotropic resolution black blood MRI at 3.0 Tesla. Journal of Magnetic Resonance Imaging : JMRI 2011;34:22-30. 


\section{CHAPTER 3}

18. Zhu C, Haraldsson $\mathrm{H}$, Tian $\mathrm{B}$, et al. High resolution imaging of the intracranial vessel wall at 3 and 7 T using 3D fast spin echo MRI. MAGMA 2016;29:559-70.

19. Xie Y, Yang Q, Xie G, Pang J, Fan Z, Li D. Improved black-blood imaging using DANTESPACE for simultaneous carotid and intracranial vessel wall evaluation. Magnetic Resonance in Medicine 2016;75:2286-94.

20. Wang J, Helle M, Zhou Z, Bornert P, Hatsukami TS, Yuan C. Joint blood and cerebrospinal fluid suppression for intracranial vessel wall MRI. Magnetic Resonance in Medicine 2016;75:831-8.

21. Zhang L, Zhang $N$, Wu J, Huang $Y$, Liu $X$, Chung YC. High resolution three dimensional intracranial arterial wall imaging at $3 \mathrm{~T}$ using T1 weighted SPACE. Magnetic Resonance Imaging 2015;33:1026-34.

22. Li ML, Xu YY, Hou B, et al. High-resolution intracranial vessel wall imaging using 3D CUBE T1 weighted sequence. European Journal of Radiology 2016;85:803-7.

23. Busse RF, Brau ACS, Vu A, Michelich CR, Bayram E, Kijowski R. Effects of refocusing flip angle modulation and view ordering in $3 \mathrm{D}$ fast spin echo. Magnetic Resonance in Medicine 2008;60.

24. Weigel M, Hennig J. Diffusion sensitivity of turbo spin echo sequences. Magnetic Resonance in Medicine 2012;67:1528-37.

25. Edelman RR, Chien D, Kim D. Fast selective black blood MR imaging. Radiology 1991;181:655-60.

26. Wang J, Yarnykh VL, Yuan C. Enhanced image quality in black-blood MRI using the improved motion-sensitized driven-equilibrium (iMSDE) sequence. Journal of Magnetic Resonance Imaging : JMRI 2010;31:1256-63.

27. Ma N, Jiang WJ, Lou $X$, et al. Arterial remodeling of advanced basilar atherosclerosis: a 3-tesla MRI study. Neurology 2010;75:253-8.

28. Zhu XJ, Du B, Lou $X$, et al. Morphologic characteristics of atherosclerotic middle cerebral arteries on 3T high-resolution MRI. AJNR American Journal of Neuroradiology 2013;34:1717-22.

29. Gutierrez J, Goldman J, Honig LS, Elkind MS, Morgello S, Marshall RS. Determinants of cerebrovascular remodeling: do large brain arteries accommodate stenosis? Atherosclerosis 2014;235:371-9.

30. Qiao Y, Anwar Z, Intrapiromkul J, et al. Patterns and Implications of Intracranial Arterial Remodeling in Stroke Patients. Stroke 2016;47:434-40.

31. Swartz RH, Bhuta SS, Farb RI, et al. Intracranial arterial wall imaging using high-resolution 3-tesla contrast-enhanced MRI. Neurology 2009;72:627-34.

32. Fan Z, Yang Q, Deng Z, et al. Whole-brain intracranial vessel wall imaging at 3 Tesla using cerebrospinal fluid-attenuated T1-weighted 3D turbo spin echo. Magnetic Resonance in Medicine 2017;77:1142-1150.

33. Yang H, Zhang X, Qin Q, Liu L, Wasserman BA, Qiao Y. Improved cerebrospinal fluid suppression for intracranial vessel wall MRI. Journal of Magnetic Resonance Imaging : JMRI 2016;44:665-72. 
34. Gutierrez J, Elkind MS, Petito C, Chung DY, Dwork AJ, Marshall RS. The contribution of HIV infection to intracranial arterial remodeling: a pilot study. Neuropathology 2013;33: 256-63.

35. Qiao Y, Guallar E, Suri FK, et al. MR Imaging Measures of Intracranial Atherosclerosis in a Population-based Study. Radiology 2016;280:860-8.

36. Dobrin PB. Effect of histologic preparation on the cross-sectional area of arterial rings. The Journal of Surgical Research 1996;61:413-5.

37. Tran T, Sundaram CP, Bahler CD, et al. Correcting the Shrinkage Effects of Formalin Fixation and Tissue Processing for Renal Tumors: toward Standardization of Pathological Reporting of Tumor Size. Journal of Cancer 2015;6:759-66.

38. Bouvy WH, Biessels GJ, Kuijf HJ, Kappelle LJ, Luijten PR, Zwanenburg JJ. Visualization of perivascular spaces and perforating arteries with $7 \mathrm{~T}$ magnetic resonance imaging. Investigative Radiology 2014;49:307-13.

39. Lindenholz A, Harteveld AA, Siero JC, Zwanenburg J, Hendrikse J. A multiple comparison between $3 T$ intracranial vessel wall sequences. Proceedings of the 24th Annual Meeting of ISMRM Singapore 2016: [Abstract 1065] 2016.

40. Ryu CW, Jahng GH, Kim EJ, Choi WS, Yang DM. High resolution wall and lumen MRI of the middle cerebral arteries at 3 tesla. Cerebrovasc Dis 2009;27:433-42.

41. Lee WJ, Choi HS, Jang J, et al. Non-stenotic intracranial arteries have atherosclerotic changes in acute ischemic stroke patients: a 3T MRI study. Neuroradiology 2015;57:1007-13.

42. Dieleman N, Yang W, Abrigo JM, et al. Magnetic Resonance Imaging of Plaque Morphology, Burden, and Distribution in Patients With Symptomatic Middle Cerebral Artery Stenosis. Stroke; 2016; 47:1797-1802.

43. Dieleman N, Yang W, van der Kolk AG, et al. Qualitative Evaluation of a High-Resolution 3D Multi-Sequence Intracranial Vessel Wall Protocol at 3 Tesla MRI. PloS one 2016;11:e0160781.

44. Xu W-H, Li M-L, Gao S, Ni J, Zhou L-X, Yao M. In vivo high-resolution MR imaging of symptomatic and asymptomatic middle cerebral artery atherosclerotic stenosis. Atherosclerosis 2010;212:507-11.

45. Harteveld AA, van der Kolk AG, van der Worp HB, et al. High-resolution intracranial vessel wall MRI in an elderly asymptomatic population: comparison of 3T and 7T. European Radiology 2017;27:1585-1595.

46. Oppenheim C, Naggara O, Touze E, et al. High-resolution MR imaging of the cervical arterial wall: what the radiologist needs to know. Radiographics 2009;29:1413-31.

47. Harteveld AA, Denswil NP, Siero JC, et al. Quantitative Intracranial Atherosclerotic Plaque Characterization at 7T MRI: An Ex Vivo Study with Histologic Validation. AJNR American Journal of Neuroradiology 2016;37:802-10.

48. Van der Kolk AG, Hendrikse J, Brundel M, Biessels GJ, Smit EJ, Visser F. Multi-sequence whole-brain intracranial vessel wall imaging at 7.0 tesla. European Radiology 2013;23:2996-3004. 


\section{CHAPTER 3}

49. Teeuwisse WM, Brink WM, Webb AG. Quantitative assessment of the effects of high-permittivity pads in 7 Tesla MRI of the brain. Magnetic Resonance in Medicine 2012;67:1285-93.

50. Ellingson BM, Bendszus $M$, Boxerman J, et al. Consensus recommendations for a standardized Brain Tumor Imaging Protocol in clinical trials. Neuro-oncology 2015;17:1188-98.

51. Fayad ZA. MR imaging for the noninvasive assessment of atherothrombotic plaques. Magnetic Resonance Imaging Clinics of North America 2003;11:101-13.

52. Harteveld AA, De Cocker LJ, Dieleman N, et al. High-resolution postcontrast time-of-flight MR angiography of intracranial perforators at 7.0 Tesla. PloS one 2015;10:e0121051.

53. Van der Kolk AG, Zwanenburg JJ, Brundel M, et al. Distribution and natural course of intracranial vessel wall lesions in patients with ischemic stroke or TIA at 7.0 Tesla MRI. European Radiology 2015;25:1692-700.

54. Seo WK, Oh K, Suh SI, Seol HY. Clinical Significance of Wall Changes After Recanalization Therapy in Acute Stroke: High-Resolution Vessel Wall Imaging. Stroke 2017;48:10771080.

55. Power S, Matouk C, Casaubon LK, et al. Vessel wall magnetic resonance imaging in acute ischemic stroke: effects of embolism and mechanical thrombectomy on the arterial wall. Stroke 2014;45:2330-4.

56. Qiao Y, Zeiler SR, Mirbagheri S, et al. Intracranial plaque enhancement in patients with cerebrovascular events on high-spatial-resolution MR images. Radiology 2014;271:53442.

57. De Rotte AA, van der Kolk AG, Rutgers $D$, et al. Feasibility of high-resolution pituitary MRI at 7.0 tesla. European Radiology 2014;24:2005-11.

58. Portanova A, Hakakian N, Mikulis DJ, Virmani R, Abdalla WM, Wasserman BA. Intracranial vasa vasorum: insights and implications for imaging. Radiology 2013;267:667-79.

59. Morelli JN, Runge VM, Ai F, et al. An image-based approach to understanding the physics of MR artifacts. Radiographics 2011;31:849-66.

60. Kontzialis M, Wasserman BA. Intracranial vessel wall imaging: current applications and clinical implications. Neurovascular Imaging 2016;2:1-6.

61. De Havenon A, Chung L, Park M, Mossa-Basha M. Intracranial vessel wall MRI: a review of current indications and future applications. Neurovascular Imaging 2016;2:10.

62. Bhogal P, Navaei E, Makalanda HL, et al. Intracranial vessel wall MRI. Clinical radiology 2016;71:293-303.

63. Lustig M, Donoho D, Pauly JM. Sparse MRI: The application of compressed sensing for rapid MR imaging. Magnetic resonance in medicine 2007;58:1182-95.

64. Li B, Li H, Li J, et al. Relaxation enhanced compressed sensing three-dimensional black-blood vessel wall MR imaging: Preliminary studies. Magnetic Resonance Imaging 2015;33:932-8. 
65. Li L, Miller KL, Jezzard P. DANTE-prepared pulse trains: a novel approach to motionsensitized and motion-suppressed quantitative magnetic resonance imaging. Magnetic Resonance in Medicine 2012;68:1423-38.

66. Hu X, Li Y, Zhang L, Zhang X, Liu X, Chung YC. A 32-channel coil system for MR vessel wall imaging of intracranial and extracranial arteries at 3T. Magnetic Resonance Imaging 2017;36:86-92.

67. Majidi S, Sein J, Watanabe M, et al. Intracranial-derived atherosclerosis assessment: an in vitro comparison between virtual histology by intravascular ultrasonography, 7T MRI, and histopathologic findings. AJNR American Journal of Neuroradiology 2013;34:2259-64.

68. Turan TN, Rumboldt Z, Granholm AC, et al. Intracranial atherosclerosis: correlation between in-vivo 3T high resolution MRI and pathology. Atherosclerosis 2014;237:460-3.

69. Kleinloog R, Korkmaz E, Zwanenburg JJ, et al. Visualization of the aneurysm wall: a 7.0-tesla magnetic resonance imaging study. Neurosurgery 2014;75:614-22.

70. Van der Kolk AG, Zwanenburg JJ, Denswil NP, et al. Imaging the intracranial atherosclerotic vessel wall using 7T MRI: initial comparison with histopathology. AJNR American Journal of Neuroradiology 2015;36:694-701.

71. Jiang $Y$, Zhu C, Peng W, et al. Ex-vivo imaging and plaque type classification of intracranial atherosclerotic plaque using high resolution MRI. Atherosclerosis 2016;249:10-6.

72. Li ML, $\mathrm{Xu} W H$, Song $\mathrm{L}$, et al. Atherosclerosis of middle cerebral artery: evaluation with high-resolution MR imaging at 3T. Atherosclerosis 2009;204:447-52.

73. Mandell DM, Matouk CC, Farb RI, et al. Vessel wall MRI to differentiate between reversible cerebral vasoconstriction syndrome and central nervous system vasculitis: preliminary results. Stroke; a journal of cerebral circulation 2012;43:860-2.

74. Obusez EC, Hui F, Hajj-Ali RA, Cerejo R, Calabrese LH, Hammad T. High-resolution MRI vessel wall imaging: spatial and temporal patterns of reversible cerebral vasoconstriction syndrome and central nervous system vasculitis. AJNR American Journal of Neuroradiology 2014;35:1527-32.

75. Kim JM, Jung KH, Sohn CH, Park J, Moon J, Han MH. High-resolution MR technique can distinguish moyamoya disease from atherosclerotic occlusion. Neurology 2013;80.

76. Mossa-Basha M, de Havenon A, Becker KJ, et al. Added Value of Vessel Wall Magnetic Resonance Imaging in the Differentiation of Moyamoya Vasculopathies in a Non-Asian Cohort. Stroke 2016;47:1782-8.

77. Ryoo S, Cha J, Kim SJ, Choi JW, Ki CS, Kim KH. High-resolution magnetic resonance wall imaging findings of Moyamoya disease. Stroke 2014;45.

78. Aoki S, Hayashi N, Abe O, Shirouzu I, Ishigame K, Okubo T. Radiation-induced arteritis: thickened wall with prominent enhancement on cranial MR images report of five cases and comparison with 18 cases of Moyamoya disease. Radiology 2002;223.

79. Yu LB, Zhang Q, Shi ZY, Wang MQ, Zhang D. High-resolution Magnetic Resonance Imaging of Moyamoya Disease. Chinese Medical Journal 2015;128:3231-7. 
80. Arai D, Satow T, Komuro T, Kobayashi A, Nagata H, Miyamoto S. Evaluation of the Arterial Wall in Vertebrobasilar Artery Dissection Using High-Resolution Magnetic Resonance Vessel Wall Imaging. Journal of Stroke and Cerebrovascular Diseases 2016;25:1444-50.

81. Wang $Y$, Lou $X$, Li $Y$, et al. Imaging investigation of intracranial arterial dissecting aneurysms by using $3 \mathrm{~T}$ high-resolution MRI and DSA: from the interventional neuroradiologists' view. Acta neurochirurgica 2014;156:515-25.

82. Bachmann R, Nassenstein I, Kooijman $\mathrm{H}$, et al. High-resolution magnetic resonance imaging (MRI) at 3.0 Tesla in the short-term follow-up of patients with proven cervical artery dissection. Investigative Radiology 2007;42:460-6.

83. Fiebach J, Brandt $T$, Knauth M, Jansen O. [MRI with fat suppression in the visualization of wall hematoma in spontaneous dissection of the internal carotid artery]. RoFo: Fortschritte auf dem Gebiete der Rontgenstrahlen und der Nuklearmedizin 1999;171:290-3.

84. Nagahata S, Nagahata M, Obara M, etal. Wall Enhancement of the Intracranial Aneurysms Revealed by Magnetic Resonance Vessel Wall Imaging Using Three-Dimensional Turbo Spin-Echo Sequence with Motion-Sensitized Driven-Equilibrium: A Sign of Ruptured Aneurysm? Clinical Neuroradiology 2016:26:277-83.

85. Edjlali M, Gentric JC, Regent-Rodriguez C, Trystram D, Hassen WB, Lion S. Does aneurysmal wall enhancement on vessel wall MRI help to distinguish stable from unstable intracranial aneurysms? Stroke 2014;45:3704-6.

86. Matouk CC, Mandell DM, Gunel M, Bulsara KR, Malhotra A, Hebert R. Vessel wall magnetic resonance imaging identifies the site of rupture in patients with multiple intracranial aneurysms: proof of principle. Neurosurgery 2013;72:492-6.

87. Kondo R, Yamaki T, Mouri W, et al. [Magnetic resonance vessel wall imaging reveals rupture site in subarachnoid hemorrhage with multiple cerebral aneurysms]. No shinkei geka Neurological Surgery 2014;42:1147-50.

88. Ducros A. Reversible cerebral vasoconstriction syndrome. The Lancet Neurology 2012;11:906-17.

89. Wang J, Guan M, Yamada K, et al. In Vivo Validation of Simultaneous Non-Contrast Angiography and intraPlaque Hemorrhage (SNAP) Magnetic Resonance Angiography: An Intracranial Artery Study. PloS one 2016;11:e0149130.

90. Park JK, Kim SH, Kim BS, Choi G, Jeong SY, Choi JC. Imaging of intracranial plaques with black-blood double inversion recovery MR imaging and CT. Journal of Neuroimaging 2011;21:e64-8.

91. Chen XY, Wong KS, Lam WW, Ng HK. High signal on T1 sequence of magnetic resonance imaging confirmed to be intraplaque haemorrhage by histology in middle cerebral artery. International Journal of Stroke 2014;9:E19.

92. Xu WH, Li ML, Gao S, et al. Middle cerebral artery intraplaque hemorrhage: prevalence and clinical relevance. Annals of Neurology 2012;71:195-8.

93. Turan TN, Bonilha L, Morgan PS, Adams RJ, Chimowitz MI. Intraplaque hemorrhage in symptomatic intracranial atherosclerotic disease. Journal of Neuroimaging 2011;21:e159-61. 
94. Chen XY, Wong KS, Lam WW, Zhao HL, Ng HK. Middle cerebral artery atherosclerosis: histological comparison between plaques associated with and not associated with infarct in a postmortem study. Cerebrovasc Dis 2008;25:74-80. 


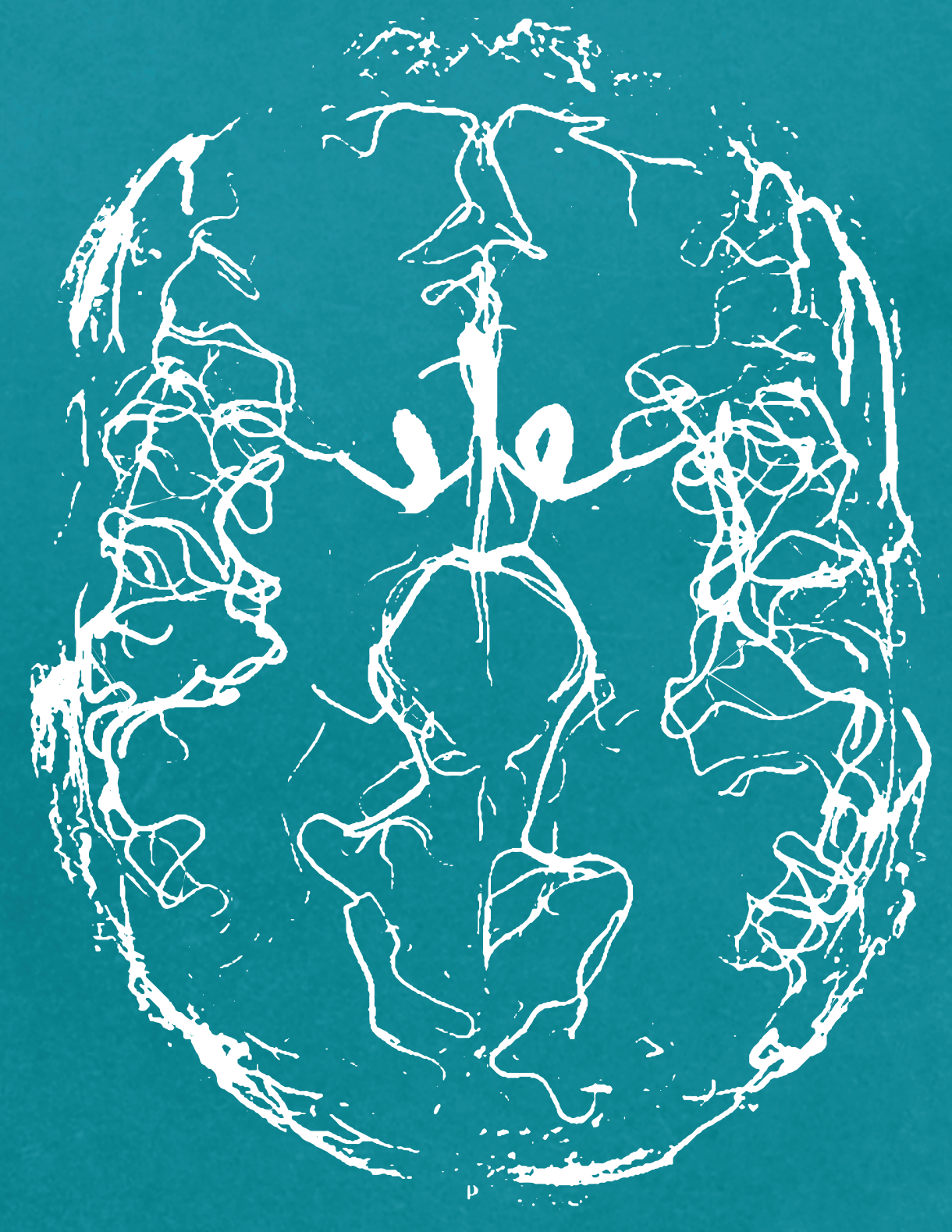




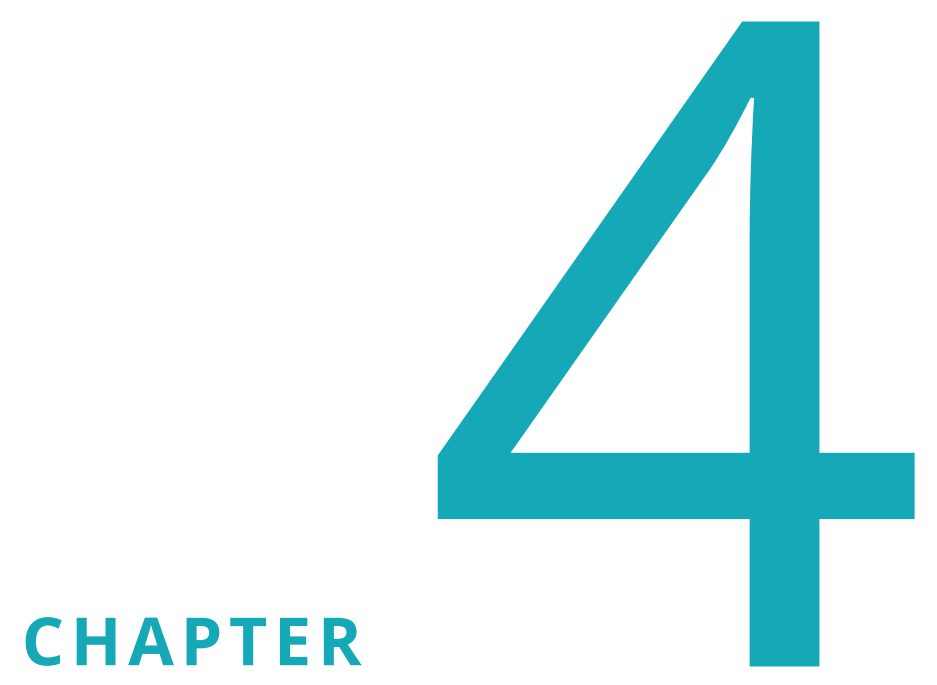

\section{Comparison of 3T Intracranial Vessel Wall MRI Sequences}

Arjen Lindenholz ${ }^{1}$, Anita A. Harteveld ${ }^{1}$, Jaco J.M. Zwanenburg ${ }^{1}$, Jeroen C.W. Siero ${ }^{1,2}$ and J. Hendrikse ${ }^{1}$

${ }^{1}$ Department of Radiology, University Medical Center Utrecht, The Netherlands ${ }^{2}$ Spinoza Center for Neuroimaging, Amsterdam, The Netherlands Published in American Journal of Neuroradiology (AJNR), 2018; 39 (6) 1112-1120. 


\begin{abstract}
Background and Purpose: Intracranial vessel wall MRI plays an increasing role in diagnosing intracranial vascular diseases. For a complete assessment, pre- and postcontrast sequences are required and, including other sequences, this results in a long scan duration. Ideally, the scan time of the vessel wall sequence should be reduced. The purpose of this study was to evaluate different intracranial vessel wall sequence variants to reduce scan duration, provided an acceptable image quality can be maintained.
\end{abstract}

Materials and Methods: Starting from the vessel wall sequence that we use clinically ( 6 minutes 42 seconds), 6 scan variants were tested (scan duration ranging between 4 minutes 39 seconds to 8 minutes 24 seconds), creating various trade-offs among spatial resolution, signal-to-noise-ratio (SNR) and contrast-to-noise-ratio (CNR). In total, 15 subjects were scanned on a 3T MRI scanner; in 5 subjects all 7 variants were performed precontrast-only and in 10 other subjects, the fastest variant (4 minutes 39 seconds) and our clinically used variant ( 6 minutes 42 seconds) were performed pre- and postcontrast.

Results: The fastest variant (4 minutes 39 seconds) had higher or comparable SNRs/CNRs of the intracranial vessel walls compared with the reference sequence (6 minutes 42 seconds). Qualitative assessment showed cerebrospinal fluid was most suppressed in the fastest variant of 4 minutes 39 seconds and the variant of 6 minutes 42 seconds. Pre- and postcontrast, SNRs/CNRs of the fastest variant were all, except one, higher compared with the variant of 6 minutes 42 seconds ( $p<0.008$ ). Furthermore, the fastest variant (4 minutes 39 seconds) detected all vessel wall lesions identified on the 6 minutes 42 seconds variant.

Conclusions: A 30\% faster vessel wall sequence was developed with high SNRs/ CNRs that resulted in good visibility of the intracranial vessel wall. 


\section{Introduction}

High-resolution intracranial vessel wall magnetic resonance imaging (MRI) plays an increasing role in diagnosing intracranial vascular diseases., ${ }^{1,2}$ The main advantage of this imaging technique compared with lumen-based methods such as CT-angiography and digital subtraction angiography (DSA), is the visualization of the vessel wall itself, including the detection of vessel wall lesions that do not necessarily show or only subtle luminal narrowing. ${ }^{3-7}$ Intracranial vessel wall imaging can be used for the detection and characterization of plaque burden in intracranial atherosclerotic disease, which is known to be one of the most important contributing factors of ischemic stroke and may be detected in an early stage. ${ }^{8-10}$ It can also be helpful in the differentiation of other vascular diseases, such as vasculitis and reversible vasoconstriction syndrome, with, for instance, visualization of vessel wall enhancement. ${ }^{11}$ Furthermore, intracranial vessel wall imaging may aid in the diagnosis of aneurysm rupture and intracranial dissection, though this is less supported in the literature..$^{12-14}$

Intracranial vessel wall MRI requires a high spatial resolution to visualize the thin vessel wall and potential accompanying vessel wall lesions. Currently, most 3D-acquired vessel wall sequences use a voxel size between 0.4 and $0.7 \mathrm{~mm}$, though this is larger than the normal diameter of the intracranial vessel walls.,15 In addition, a high signal-to-noise-ratio (SNR) and contrast-to-noise-ratio (CNR) are required to delineate the vessel wall from surrounding tissue (i.e., blood, cerebrospinal fluid (CSF) and parenchyma). ${ }^{1,2,16}$ At higher magnetic field strengths, a higher spatial resolution and/or SNR can be achieved. Therefore, vessel wall MR imaging is currently performed at $3 \mathrm{~T}$ and higher field strengths. However, with high spatial resolution, the SNR is still limited and the total scan duration is long. Recently published intracranial vessel wall MRI sequences have scan durations ranging from 5.0 to 10.2 minutes. ${ }^{16-22}$ To assess vessel wall lesions, one needs pre- and postcontrast acquisitions to evaluate contrast enhancement of the vessel walls. For a complete examination, other sequences, such as Time-of-Flight (TOF) MR angiography, Diffusion Weighted Imaging (DWI) and $\mathrm{T}_{2}$-Fluid-attenuated Inversion-Recovery (FLAIR) images may also be needed, resulting in a long total scan duration. With longer scan durations, motion artifacts may increase, especially in neurologically impaired patients. Ideally, total scan duration of the pre- and postcontrast vessel wall sequence should be reduced without sacrificing image quality. In this study, SNR and CNR of one earlier reported intracranial vessel wall sequence ${ }^{23,24}$ was compared with 6 variations, which includes another previously reported sequence, ${ }^{19}$ with different trade-offs between scan duration, resolution, and contrast. Subsequently, pre- and postcontrast images of the fastest vessel wall variant were compared with the current variant that is used in our daily clinical practice. ${ }^{24}$ 


\section{Materials and Methods}

\section{Participants}

Fifteen subjects were included in this study. In 5 subjects (4 male; mean age 37 years, range 28-54 years) all 7 scan variants were performed (precontrast-only). Additionally, precontrast and postcontrast comparisons were performed in 10 other subjects ( 9 male; mean age 53 years, range 24-68 years). Approval for this study was obtained from the Institutional Review Board of University Medical Center Utrecht. The 10 volunteers who received contrast agent were part of the PIVI-study and IVI-study (Posterior Intracranial Vessel wall Imaging and Intracranial Vessel wall Imaging; NTR5688 and NTR2119, respectively, www.trialregister.nl). All subjects gave written informed consent.

\section{Imaging protocol}

We used a 3T MRI-system (Achieva, Philips Healthcare, Best, The Netherlands) with an 8-channel phased-array sensitivity encoding (SENSE) head coil. All vessel wall sequences consisted of a 3D fast turbo spin-echo (TSE) sequence and a number of acquisitions (NSA) of 1 . Starting from our earlier described vessel wall sequence (variant 3 in Table 1), ${ }^{23}$ six different variants were created, of which one is previously described (variant 2$).{ }^{19}$ In the scan protocol of the precontrast-only acquisitions, the sequence acquisition order was equal in each subject (consecutively variant 2, 5, 3, 4, 7, 1 and 6; Table 1). Variant 3 was used as reference sequence for the 6 different precontrast-only acquisitions. Various trade-offs were created among contrast (repetition time (TR), Anti-Driven Equilibrium), spatial resolution (voxelsize, elliptical $k$-space shutter), SNR (oversample factor in phase-encoding direction, SENSitivity Encoding (SENSE) reduction factor, readout bandwidth, overcontiguous (overlapping) slices, TSE-train flip-angle refocusing control), and scan duration (Table 1). All vessel wall scans were repeated with the radiofrequency and gradient turned off for sampling noise images from the receiver coil elements, including image reconstruction effects such as elliptical $k$-space shutter and parallel (SENSE) reconstruction sensitivities. The noise images were accelerated by leaving out the delay after each TSE train to the next (turned off) excitation. The total scan session took 65 minutes and included the preparation phase, TOF-MRA (acquired voxel size $0.4 \times 0.7 \times 1.0 \mathrm{~mm}^{3}$, reconstructed voxel size $0.36 \times 0.36 \times 0.5 \mathrm{~mm}^{3}$, acquisition time 5 minutes 12 seconds) and the 7 vessel wall scan variants, including their corresponding noise images. For the vessel wall sequences, the field-of-view (FOV) was planned in a transverse / oblique orientation, which covered all large cerebral arteries of the circle of Willis. 
In addition, in 10 other subjects precontrast and postcontrast images were acquired of our earlier published and clinically used variant 3 , which had good CSF suppression, and the fastest, variant 7 (consecutively variant 3 and 7 in 8 subjects and consecutively variant 7 and 3 in 2 subjects). We have chosen to compare the fastest, variant 7, with our clinical used variant for clinical and practical purposes. Before acquisition of the postcontrast sequences, a gadolinium-containing contrast agent (gadobutrol, Gadovist $1.0 \mathrm{mmol} / \mathrm{mL}$, Bayer Schering Pharma, Berlin, Germany), was administered intravenously. The total scan session took 45 minutes and included, consecutively, the preparation phase with the survey scans, TOFMRA, precontrast vessel wall sequences, postcontrast DWI, postcontrast vessel wall sequences (acquired $\sim 20$ min after the precontrast vessel wall sequence) including noise images; and finally, a postcontrast T2-FLAIR sequence. 


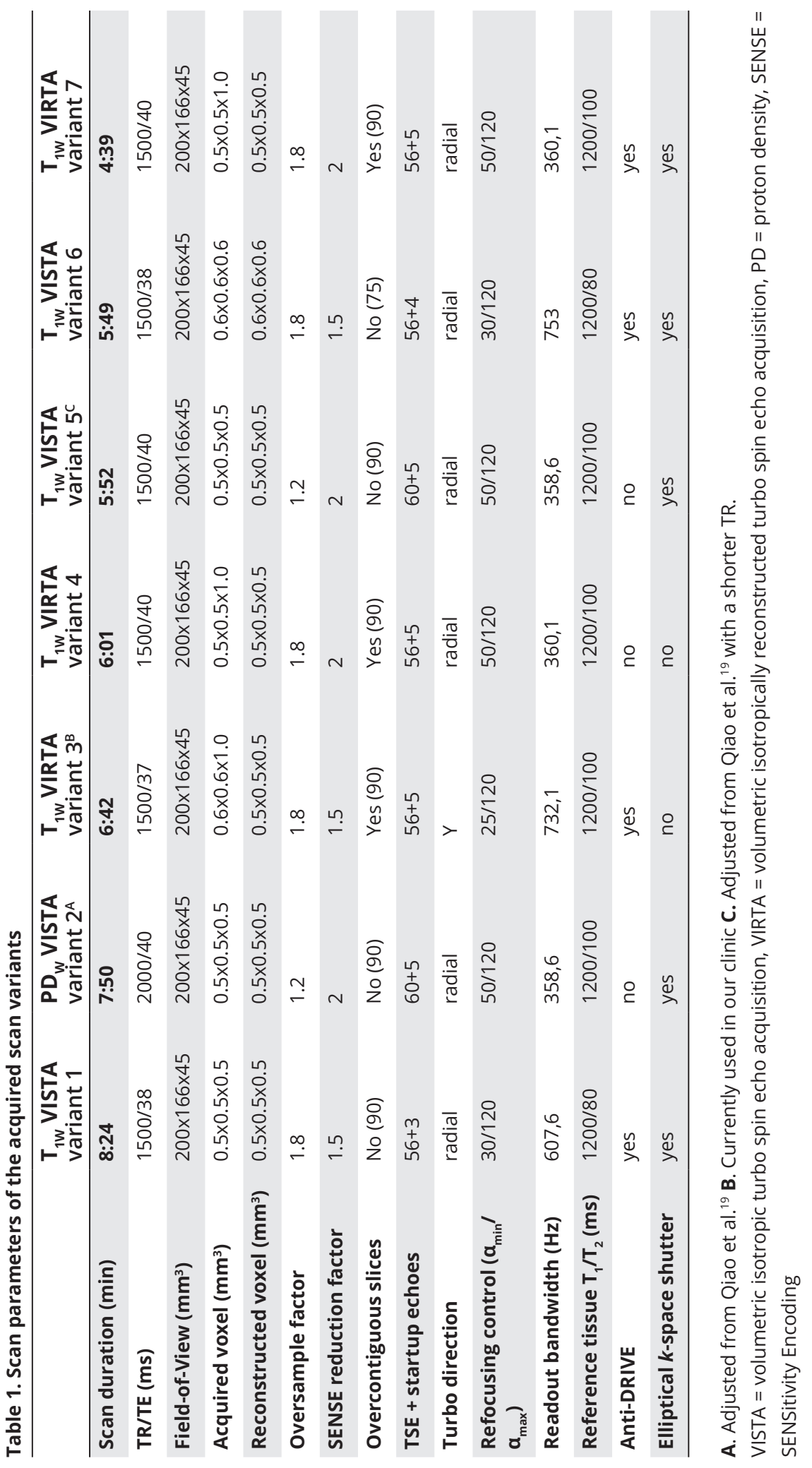




\section{Image analysis}

The mean signal and standard deviation of the noise $\left(\mathrm{SD}_{\text {noise }}\right)$ were obtained from an axial plane of the vessel wall images and noise images (magnitude), respectively. Regions of interest (ROIs) for the mean signal $\left(\right.$ Mean $\left._{\mathrm{RO}}\right)$ were manually drawn as follows: CSF was marked in the suprasellar and pontine cistern for the intracranial internal carotid arteries and basilar artery, respectively; blood was marked in the lumen of the middle cerebral artery; brain tissue was marked in the left orbital gyrus; and the vessel wall was represented by the circumferential of the intracranial internal carotid arteries (left and right) and basilar artery (within the same slice number as the ROIs for CSF; Figure 1). In the 10 subjects who received contrast agent injection, a homogeneous hyperintense part of the center of the pituitary stalk was used as ROI to compute the mean as a consistent marker for contrast-enhancement (Figure 1). To compute the SD of the noise images ( $S D_{\text {noise }}$, a larger circular ROI was drawn on the noise images, encompassing the ROls used to compute the mean. The SNR was calculated as SNR $=$ Mean $_{\text {ROI }} / \mathrm{SD}_{\text {noise }}$ for each subject and subsequently averaged over all subjects included in the comparison. The CNRs were calculated as $\mathrm{CNR}_{\mathrm{x}-\mathrm{y}}=\mathrm{SNR}_{\mathrm{x}}-\mathrm{SNR}_{\mathrm{y}}$. As a measure of motion, vessel wall sequences were coregistered to the prior acquired sequence to calculate the registration parameters ( $\Delta$ Rotation and $\Delta$ Translation), using the elastix toolbox in MeVisLab (version 2.7; MeVis Medical Solutions, Bremen, Germany). ${ }^{25}$
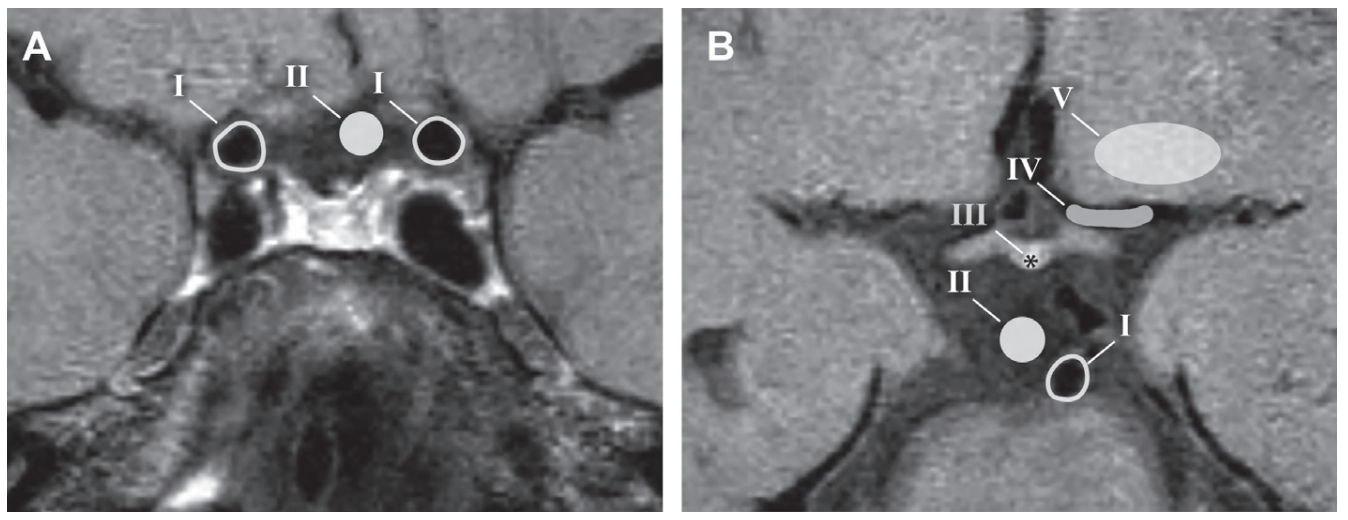

Figure 1. ROls used in the magnitude images for calculating the signal-to-noise-ratios. (A) The circumferential of both carotid arteries (I) was used as marker for the carotid vessel wall, that runs through the suprasellar cistern which was used as marker for CSF (II). (B) The circumferential of the basilar artery (I) was used as marker for the basilar vessel wall that run through the pontine cistern, which was used as second marker for CSF (II). For the pituitary stalk, a homogenous hyperintense part of the center was used (III). In the lumen of the middle cerebral artery a ROI was drawn as a marker for blood (IV), and in the left orbital gyrus a ROI was drawn as marker for brain tissue (V). The ROls are drawn for illustrative purposes and the exact contours may differ in the real measurements depending on patients' specific anatomy. $R O I=$ region of interest, $C S F=$ cerebrospinal fluid. 
An expert neuroradiologist $(\mathrm{HH}$ with $>15$ year of experience), specialized in intracranial vessel wall imaging, assessed both pre- and postcontrast vessel wall images in multiple planes for image quality, which includes the visibility of the arterial vessel wall, the suppression of blood and CSF and the existence of artifacts (slow-flow, motion and free induction decay (FID) artifacts). The images of the 10 subjects who received contrast agent injection were also assessed for the presence of vessel wall lesions, including location and configuration (eccentric or concentric) and contrast-enhancement using methods previously described. 24,26 All scans were blinded and randomly ordered before assessment. When a lesion was found in only one of the variants, the location was re-examined in the other variant to check if the lesion could be identified retrospectively. Furthermore, the vessel wall images were cross-correlated with the TOF-MRA images for a correct interpretation of the specific arteries, the potential vessel wall lesions and the lumen of the artery. Included vessels were the anterior cerebral arteries (A1 and A2 segments), distal intracranial internal carotid arteries (clinoid or $\mathrm{C} 5$ and cavernous or C4 segments), middle cerebral arteries (M1, M2 and M3 segments), posterior cerebral arteries (P1 and P2 segment), the distal intracranial basilar artery, and distal intracranial vertebral arteries.

\section{Statistical analysis}

All statistical comparisons were conducted using IBM SPSS statistics (version 21, IBM Corp., Armonk, NY, USA). Pairwise comparisons were performed between the clinically relevant SNRs and CNRs of the reference variant and the 6 other variants using generalized estimation equations to account for repeated measures on the same subjects. A Friedman test was performed for differences in SNR and CNR between both precontrast and postcontrast images of variant 3 and 7 . A post hoc analysis with the Wilcoxon signed-rank tests was conducted with a Bonferroni correction applied to correct for multiple comparisons. A p-value $<0.008$ was considered significant.

\section{Results}

All 15 subjects underwent the MRI examination successfully. The quality of all images was sufficient to be used for analysis. Image assessment was not hampered by motion artifacts in any of the scanned subjects.

\section{Comparison of all seven scan variants}

Typical images of the 7 vessel wall scan variants are shown in Figure $\mathbf{2}$ (axial plane), Figure $\mathbf{3}$ (sagittal plane) and Supplemental Figure $\mathbf{1}$ (coronal plane). The images show clear differences among the variants in contrast and visibility of the vessel 
wall. Qualitative assessment of the images in axial plane shows poor visibility of the vessel wall in variant 1 ( 8 minutes 24 seconds) and 6 (5 minutes 49 seconds), acquired with an isotropic voxel-size. Variant 4 (6 minutes 01 seconds) and 7 (4 minutes 39 seconds), which were acquired with an anisotropic voxel-size, had a higher qualitative assessment score compared with the other variants. Qualitative assessment of the axial, sagittal and coronal images showed that CSF was most suppressed in variant 3 ( 6 minutes 42 seconds) and 7 ( 4 minutes 39 seconds). These 2 variants were compared before and after contrast administration in the next paragraph (Table 2). Variant 1 (8 minutes 24 seconds) also had an adequate CSF suppression, but the axial and sagittal images had an overall granular appearance, which made assessment and delineation of the vessel wall with their surrounding CSF challenging. This granular appearance was also seen to a lesser extent in variant 5 ( 5 minutes 52 seconds) and 6 ( 5 minutes 49 seconds), but with a worse CSF suppression. In the axial plane the vessel wall was best seen in variant 2 ( 7 minutes 50 seconds), 3 ( 6 minutes 42 seconds), 4 ( 6 minutes 01 seconds) and 7 ( 4 minutes 49 seconds). In both the sagittal and coronal planes, the vessel wall is of highest quality in the isotropic variant 2 (7 minutes 50 seconds), though the contrast with the surrounding CSF is less, which makes delineation of the outer margins of the vessel wall more difficult. Due to the granular appearance, the perpendicular visibility (sagittal plane) of the middle cerebral artery was poor in variants 1 ( 8 minutes 24 seconds) and 6 ( 5 minutes 49 seconds). In the anisotropic variants 3 ( 6 minutes 42 seconds), 4 (6 minutes 01 seconds) and 7 (4 minutes 39 seconds), the vessel wall could be delineated in the sagittal plane, but the overall appearance was blurrier (Figure 3). All variants had sufficient blood suppression without flow artifacts, which could have limited assessment in the large intracranial arteries.

The mean SNRs and CNRs of the clinically most relevant regions, i.e., the basilar and carotid vessel wall and the surrounding CSF, are displayed in Figure 4, including the statistical comparisons with reference variant 3. The SNR and, more important, the calculated CNR results for all variants are shown in Supplemental Table 1. Relatively high SNRs of the vessel wall were measured for variant 2 (7 minutes 50 seconds), 4 ( 6 minutes 01 seconds) and 7 (4 minutes 39 seconds). In these 3 variants, the SNR of the intracranial internal carotid vessel wall was $>21$, and the SNR of the basilar vessel wall $>15$. All 7 variants showed good blood signal suppression, which resulted in most variants having a relatively high CNR between blood and the intracranial internal carotid vessel wall (CNRs ranging from 5.7 to 23, Supplemental Table 1). All except 1 variant perform borderline in the CNRs of the carotid vessel wall with the surrounding CSF (ranging from 1.3 to 3.2). Variant 7 had the highest CNR between the carotid vessel wall and the surrounding CSF (4.8). All variants performed better in the CNRs between the basilar artery vessel wall and CSF which are comparable in 6 of 7 variants (ranging from 3.6 to 5.3); only variant 4 ( 6 minutes 01 seconds) had a higher CNR (8.4), but also a large standard deviation (3.9, Figure 4). 

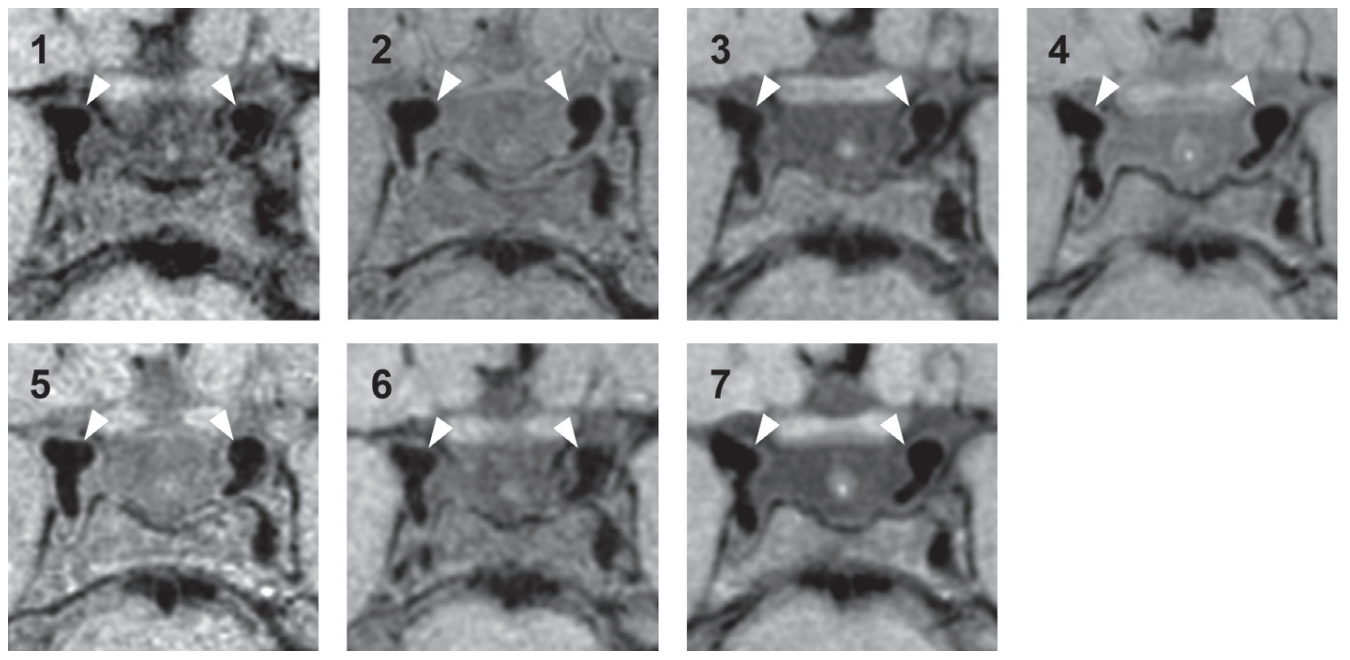

Figure 2. Sample images in the axial plane of the 7 different scan variants performed at 3T (precontrast; in order of decreasing scan duration). Both distal intracranial internal carotid arteries (white arrowheads) with the bifurcation of the posterior communicating artery are depicted surrounded by CSF. 1. $T_{1 W}$ VISTA variant 1 (8:24min). 2. PD ${ }_{w}$ VISTA variant 2 (7:50 min), adjusted from Qiao et al. ${ }^{19}{ }^{3}$. $T_{1 W}$ VIRTA variant 3 (6:42min). 4. $T_{1 W}$ VIRTA variant 4 (6.01 min). 5. $T_{1 W}$ VISTA variant 5 (5:52min), adjusted from Qiao et al. with a shorter TR. ${ }^{19}$ 6. $T_{1 \mathrm{~W}}$ VISTA variant 6 (5:49min). 7. $T_{1 \mathrm{~W}}$ VIRTA variant 7 (4:39min). VISTA = volumetric isotropic turbo-spin-echo acquisition, VIRTA = volumetric isotropically reconstructed turbo-spin echo-acquisition
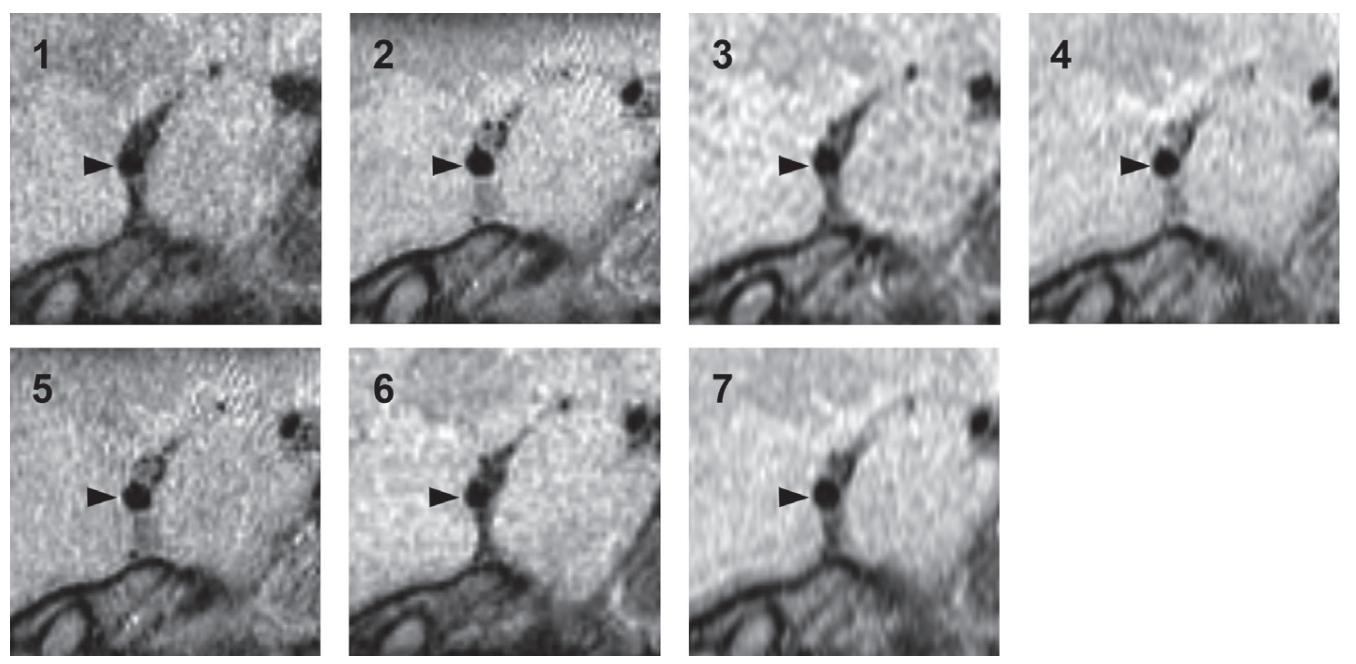

Figure 3. Sample images in sagittal plane of the 7 different scan variants performed at 3T (precontrast; in order of decreasing scan duration). The right MCA (black arrowheads) is depicted in all images, surrounded by CSF and brain parenchyma. 1. $T_{1 W}$ VISTA variant 1 (8:24min). 2. PD $\mathrm{W}_{\mathrm{W}}$ VISTA variant 2 (7:50 min), adjusted from Qiao et al. ${ }^{19}$ 3. $T_{1 \mathrm{~W}}$ VIRTA variant 3 (6:42min). 4. $T_{1 \mathrm{w}}$ VIRTA variant 4 (6.01 min). 5. $T_{1 \mathrm{~W}}$ VISTA variant 5 (5:52min), adjusted from Qiao et al. with a shorter TR. ${ }^{19}$ 6. $T_{1 \mathrm{~W}}$ VISTA variant 6 (5:49min). 7. $T_{1 \mathrm{~W}}$ VIRTA variant $7(4: 39 \mathrm{~min})$. VISTA $=$ volumetric isotropic turbo-spin-echo acquisition, VIRTA = volumetric isotropically reconstructed turbo-spin echo-acquisition 


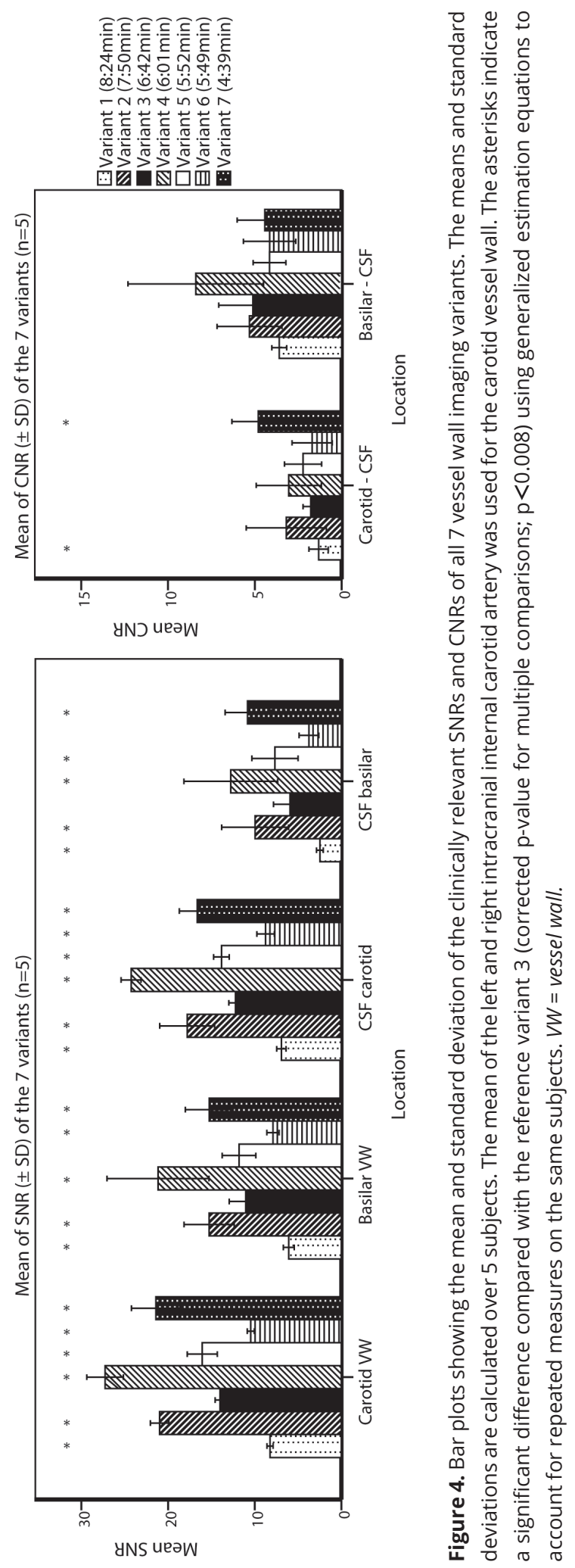




\section{Comparison of variant 3 ( 6 minutes 42 seconds) versus variant 7 (4 minutes 39 seconds)}

The average SNRs and CNRs of both precontrast and postcontrast images of variant 3 and variant 7 are shown in Table 2. Comparing SNRs and CNRs of the precontrast and postcontrast scans (Table 2) for variant 3 and 7 showed that tissue, the basilar vessel wall, suprasellar CSF of only variant 7, pontine CSF and blood SNR did not differ significantly (all p-values $>0.008$; Supplemental Table 2 ), while the SNR of the carotid vessel wall of variant $7(p=0.005)$ and the CNRs between the carotid vessel wall and CSF ( $p=0.005$ and 0.007 for variant 3 and 7, respectively) tended to be higher for the postcontrast images. Significant differences for the SNR $\mathrm{pituitary}$ gland were expected because of a strong enhancing pituitary gland on postcontrast images. For both precontrast and postcontrast vessel wall images, the SNRs and CNRs of variant 7 (4 minutes 39 seconds) were higher than those of variant 3 (6 minutes 42 seconds). These differences were all significant $(p<0.008)$, except for

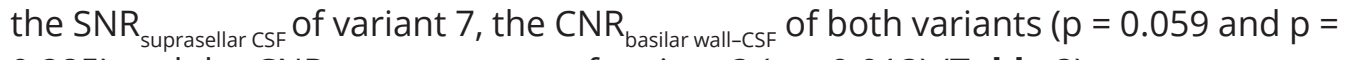
$0.285)$ and the $\mathrm{CNR}_{\text {carotid vessel wall-blood }}$ of variant $3(p=0.013)$ (Table 2$)$.

Table 2. SNRs and CNRs of the precontrast and postcontrast scan variants 3 and variant 7

\begin{tabular}{|c|c|c|c|c|c|c|}
\hline & \multicolumn{2}{|c|}{ Precontrast } & \multicolumn{4}{|c|}{ Postcontrast } \\
\hline & $\begin{array}{l}\mathbf{T}_{1 \mathrm{~W}} \text { VIRTA } \\
\text { variant } 3^{\mathrm{E}}\end{array}$ & $\begin{array}{l}\mathbf{T}_{1 \mathrm{~W}} \text { VIRTA } \\
\text { variant } 7\end{array}$ & p-value & $\begin{array}{l}\mathbf{T}_{1 \mathrm{~W}} \text { VIRTA } \\
\text { variant } 3^{\mathrm{E}}\end{array}$ & $\begin{array}{l}\mathbf{T}_{1 \mathrm{w}} \text { VIRTA } \\
\text { variant } 7\end{array}$ & p-value \\
\hline SNR $_{\text {tissue }}{ }^{A}$ & $28.0 \pm 1.8$ & $40.5 \pm 3.4$ & 0.005 & $27.9 \pm 1.3$ & $42.4 \pm 2.8$ & 0.005 \\
\hline $\mathrm{SNR}_{\text {carotid vessel wall }}{ }^{\mathrm{B}}$ & $15.3 \pm 1.4$ & $20.8 \pm 2.7$ & 0.005 & $17.2 \pm 0.9$ & $26.4 \pm 4.0$ & 0.005 \\
\hline $\mathrm{SNR}_{\text {basilary vessel wall }}$ & $13.0 \pm 1.4$ & $15.9 \pm 1.7$ & 0.005 & $13.4 \pm 2.4$ & $19.2 \pm 1.8$ & 0.005 \\
\hline SNR $_{\text {suprasellar CSF }}$ & $9.1 \pm 2.0$ & $11.9 \pm 2.2$ & 0.007 & $11.3 \pm 0.7$ & $14.1 \pm 2.4$ & 0.013 \\
\hline SNR $_{\text {pontine CSF }}$ & $5.9 \pm 2.1$ & $8.0 \pm 2.0$ & 0.007 & $6.5 \pm 1.8$ & $11.2 \pm 2.6$ & 0.007 \\
\hline SNR $_{\text {blood }}$ & $3.6 \pm 0.6$ & $5.4 \pm 1.2$ & 0.005 & $3.8 \pm 0.5$ & $6.3 \pm 1.4$ & 0.007 \\
\hline SNR $_{\text {pituitary gland }}$ & $23.0 \pm 3.4$ & $33.4 \pm 4.7$ & 0.005 & $33.3 \pm 3.5$ & $49.5 \pm 6.7$ & 0.005 \\
\hline $\mathrm{CNR}_{\text {carotid vessel wall-CSF }}{ }^{\mathrm{C}}$ & $6.1 \pm 1.6$ & $9.0 \pm 1.5$ & 0.005 & $6.0 \pm 0.7$ & $12.3 \pm 3.6$ & 0.007 \\
\hline $\mathrm{CNR}_{\text {basilary vessel wall-CSF }} \mathrm{D}$ & $7.2 \pm 1.4$ & $7.9 \pm 1.4$ & 0.059 & $7.0 \pm 2.0$ & $8.0 \pm 1.9$ & 0.285 \\
\hline $\mathrm{CNR}_{\text {carotid vessel wall -blood }}^{\mathrm{B}}$ & $11.6 \pm 1.3$ & $15.5 \pm 2.9$ & 0.013 & $13.5 \pm 1.0$ & $20.1 \pm 3.8$ & 0.007 \\
\hline
\end{tabular}

Mean and standard deviations (expressed as mean \pm SD) are calculated over 10 subjects. A Wilcoxon signed-rank test was used to compare the differences between (I) precontrast variant 3 and 7 and (II) postcontrast variant 3 and 7 (additional statistical comparisons are shown in Supplemental Table 2). A. Tissue region of interest (ROI) is located at the left orbital gyri. B. The mean of the left and right distal intracranial internal carotid artery. C. Suprasellar CSF is used as reference. D. Pontine CSF is used as reference. E. Currently used in our clinic. VIRTA = volumetric isotropically reconstructed turbo spin echo acquisition, SNR = signal-to-noise ratio, CNR = contrast-to-noise ratio, CSF = cerebrospinal fluid, Bold = statistically significant $(p<0.008)$. 
Qualitative assessment of both precontrast and postcontrast vessel wall images in multiple planes showed that in all subjects, the vessel wall could be delineated for both sequences (Figure 5). Overall, variant 7 (4 minutes 39 seconds) was slightly more affected by free induction decay (FID) artifacts in the outer regions of the FOV and slow-flow artifacts, but this did not affect the assessment of the large intracranial arteries. In one subject, variant 7 ( 4 minutes 39 seconds) showed small motion artifacts but they did not have an influence on the assessment. Sixteen vessel wall lesions were identified in variant 3 ( 6 minutes 42 seconds) and 19 in variant 7 (4 minutes 39 seconds). In 4 of 10 subjects, no vessel wall lesions were detected. Vessel wall lesions (on the basis of the 19 detected vessel wall lesions) were found in the carotid arteries $(n=3)$, middle cerebral arteries $(n=2)$, vertebral arteries $(n=10)$, basilar artery $(n=3)$ and posterior cerebral artery $(n=1)$ of which 12 were enhancing (a full description of all lesions is found in Supplemental Table 3). All 16 lesions identified in vessel wall variant 3 ( 6 minutes 42 seconds) matched vessel wall lesions identified in variant 7 (4 minutes 39 seconds) (Figure 5). One vessel wall lesion was reported as eccentric in variant 3 and concentric in variant 7, and 1 lesion, as enhancing in variant 3 and as non-enhancing in variant 7 . Three lesions were missed with vessel wall variant 3 ; one lesion located at the bifurcation of the basilar artery - P1, one at the left internal carotid artery, and one at the left vertebral artery.
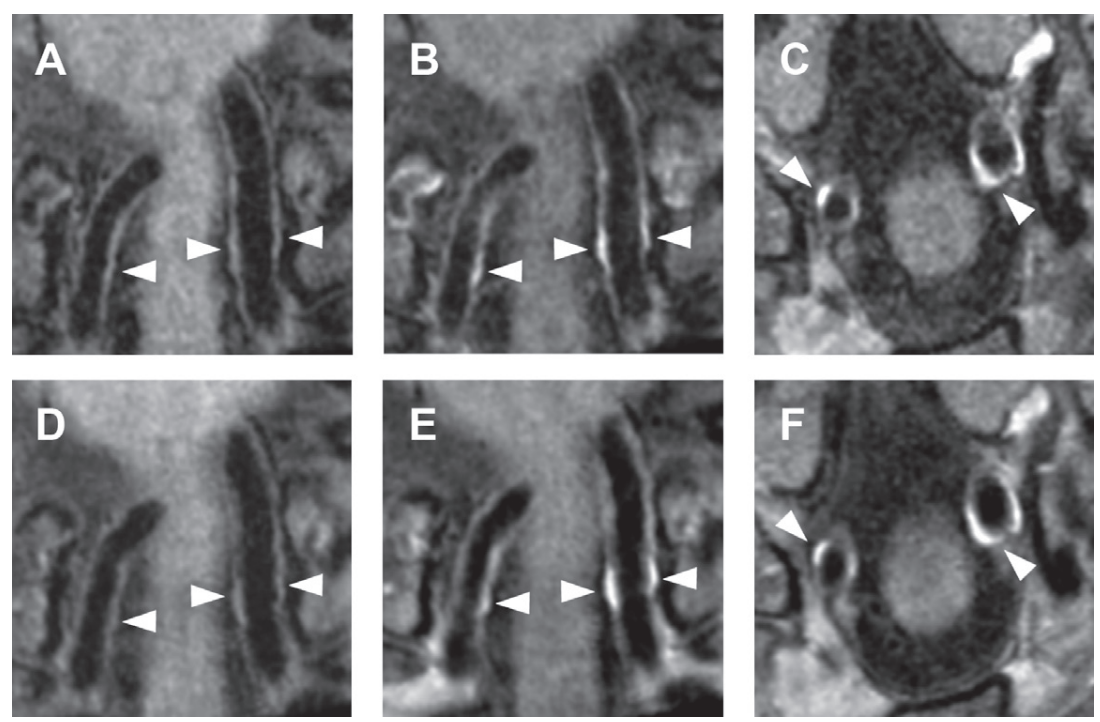

Figure 5. Matching vessel wall lesions of the distal vertebral arteries (white arrowheads) seen before and after contrast with variant 3 (6:42 min; A, B and C) and variant 7 (4:39min; D, E and F) in a 59-year-old man with multiple cardiovascular risk factors. Subtle (concentric) wall thickening is seen in both left and right vertebral arteries before contrast ( $\mathbf{A}$ and $\mathbf{D})$ with clear contrast enhancement on the postcontrast image (B and E). The variant 3 (6:42 min, B and C) was acquired approximately 2 minutes after contrast injection, and variant 7 (4:39min, E and F) approximately 9 minutes after contrast injection. Some patient motion was seen for the postcontrast series of variant 7. Postcontrast transverse images of variant $3(\mathbf{C})$ and variant 7 (F) show clear vessel wall enhancement in both vertebral arteries (white arrowheads). 


\section{Discussion}

The purpose of this study was to evaluate different variants of MRI sequences for intracranial vessel wall imaging in order to reduce scan duration, provided that an acceptable image quality could be maintained. In the pre-contrast comparison, the fastest variant 7 (4 minutes 39 seconds), had a significantly higher CNR between the vessel wall and blood, compared with variant 3, while it was about $30 \%$ faster. Also, the CNR between the vessel wall and CSF was better for variant 7 compared with variant 3. The favorable SNRs and CNRs of variant 7 also remained in the second comparison in which both precontrast and postcontrast images were acquired and were higher compared with our clinically used variant 3 (Table 2). Especially in the elderly population the superior contrast between the vessel wall and CSF can be beneficial. In the aging brain, tissue atrophy increases and consequently the intracranial vessels are more richly surrounded by CSF.

The image quality of an MR image is a balance between resolution, SNR/CNR and scan duration. A gain in one aspect, results in a sacrifice of another. The ideal balance is difficult to determine and depends on the specific application and the clinical question to be answered. Several pulse sequence optimizations are developed to address these technical challenges. ${ }^{17-19,21,26}$ For a high CNR of the vessel wall, both blood and CSF need to be simultaneously suppressed. ${ }^{1}$ 3D variable flipangle refocusing pulse sequences are used because of their high intrinsic blackblood properties and the ability to generate multiplanar reformatted images, but have less effect on the slowly flowing CSF. ${ }^{17,19-22,27}$ For better CSF suppression, Proton Density-weighted imaging with radial ordering modulation of the $k$-space (short echo-time), ${ }^{19}$ Anti-Driven Equilibrium (ADE), 18,23,28 or prepulses such as Delay Alternating with Nutation for Tailored Excitation (DANTE) ${ }^{17,21,29}$ and inversionrecovery ${ }^{30}$ have been proposed.

In 4 of 7 variants, the anti-driven equilibrium technique was used. This technique drives the magnetization back to the negative $M_{z}$-axis by further radiofrequency excitation and results in more adequate CSF suppression. ${ }^{18,24,27} \mathrm{~A}$ second possibility to improve image contrast between vessel wall and CSF is the use of a DANTE prepulse. ${ }^{17,21,29}$ When one applies DANTE pulse trains, the longitudinal magnetization of static tissue largely remains but flowing spins are suppressed due to a spoiling effect. ${ }^{29}$ This suppression is less for slow flowing fluid, especially below $0.1 \mathrm{~cm} / \mathrm{s}$; therefore, DANTE will be less useful for CSF suppression around the distal cerebral arteries. The DANTE prepulse also improves the suppression of blood, notably for slow and turbulent flow. Slow-flow is a well-known pitfall in the assessment of vessel wall imaging. ${ }^{2}$ In the current study, the postcontrast vessel wall showed a higher SNR/CNR compared with the precontrast vessel wall, which may suggest slow-slow. Another explanation that may have led to higher SNRs 
of the postcontrast vessel walls is contrast absorption into the vessel wall itself. The flow artifacts seen in variant 7 may be a potential problem and a DANTE prepulse might be a viable addition for more effective blood suppression. A third option to improve the contrast between the vessel wall and surrounding tissue is using inversion recovery. ${ }^{30}$ This technique is mainly applied in 2D acquisitions; however, also reports have been published with 3D acquisitions on a 7T MR-system. ${ }^{31}$

Generally, a lower minimum refocusing angle results in a better flow suppression but also in a lower SNR. Because of the higher minimum refocusing angles in the TSE-train of vessel wall variant 2, 4, 5 and 7 they may yield more SNRs but probably at the cost of blurring due to a worse point spread function (PSF). ${ }^{16,32}$ This may also partly explain the 'smoother' appearance in variant 4 and 7 (Figure 2). However, these sequences do show the vessel wall clearly, and in our opinion, radiologists often prefer blurring compared with images with a very narrow point spread function because this results in more noisy images which are more difficult to assess, even though the true resolution is higher.

A disadvantage of variant 3, 4 and 7 might be the anisotropic resolution in the slice direction, which makes it less beneficial for multi-planar assessment (Figure $\mathbf{2}$ and 3). ${ }^{1,19}$ In specific clinical situations, multiplanar reconstructions may be required, for example, when assessing lesions in the middle cerebral artery. However, in daily clinical practice, image interpretation can regularly be performed in the axial or oblique plane alone, and in these situations, a nearly isotropic voxel-size may be adequate for interpretation of vessel wall images. ${ }^{23,24,33}$ In addition, the scan variants with anisotropic voxel-size have a larger overall voxel-size, which results in higher SNR per voxel, but they also can offer a shorter effective scan time. On the other hand, using an anisotropic voxel-size potentially increases partial volume effects compared with the isotropic-derived variants. ${ }^{34}$ Consequently, vessel wall thickness might be overestimated or small lesions might be missed. To reduce the scan duration further, we applied an elliptical $k$-space shutter in 5 variants. Theoretically this might also reduce the image quality, because by enabling the elliptical $k$-space shutter, the outer part of the $k$-space is cut off, leading to a reduction in the effective resolution. However, in practice, the effect may be limited, particularly because the longer scan durations needed for a full acquisition make the images more prone to motion artifacts. Another way to reduce scan duration is the use of $2 \mathrm{D}$ methods as an alternative to $3 \mathrm{D}$ imaging methods. These $2 \mathrm{D}$ methods have anisotropic voxels as the slice thickness is normally much larger than the in-plane voxel-size. When these slices are planned perpendicular to the vessels, this is not a problem. However, these 2D methods have the drawback of a small coverage and need to be focused on a limited vessel wall region. The optimal region of interest may be difficult to determine beforehand. 26,35 
Besides several advanced pulse sequence optimization techniques, better hardware may also improve image quality. In the current study a relatively low number of receiver channels ( 8 channels) was used. Currently, up to 32 channels are commonly used to investigate vessel wall sequences. ${ }^{16,20,27}$ Also, when one uses a higher number of channels in de receiver coil, more possibilities, such as parallel imaging, might be available to reduce scan duration without severely compromising image quality. Moreover, parallel imaging, like SENSE, also performs better at higher field strengths. ${ }^{36}$ Another novel acceleration technique to reduce the scan duration even further may be compressed sensing. ${ }^{37}$ This technique has shown already promising results for extracranial carotid vessel wall imaging. ${ }^{38}$

This study has some limitations. First, the true image quality of an MR image is the result of a multidimensional trade-off between numerous parameters. Many of the parameters are also interrelated and may have a combined effect on resolution, SNR/CNR or scan duration and it is difficult to compare these parameters one-toone on true image quality. Furthermore, in the comparison of all 7 variants, the same order of sequences was used, which may have led to more motion artifacts in the later scheduled sequences. However, the extent of movement, measured in $\Delta$ Rotation and $\Delta$ Translation between subsequent sequences, did not increase during acquisition time (Supplemental Figure 2). Also, in the second comparison (variant 3 versus variant 7), contrast enhancement may have been stronger due to a longer absorption time in the latest scheduled variant (in our study mostly variant 7). However, statistical comparison (variant 3 and 7 versus variant 7 and 3 ) did not show any significant differences (data not shown). This study lacked a systematical comparison between the different parameters to investigate the effect on image quality. Vessel wall variants were created to reduce scan duration with the focus on development of a clinical usable sequence that can be incorporated in existing scan protocols.

The second limitation is the manually drawn ROls for measuring the SNR. Although we tried to be consistent in the location, it was not always possible to use the exact same slice number for all variants for the SNR measurement of the vessel wall and CSF, mainly due to intra-subject differences such as patient movement and inter-subject differences in planning and anatomy. Furthermore, due to flow effects of CSF in the basal cisterns, a better delineation can be made of the proximal intracranial arteries compared with the more distal intracranial arteries where the CSF flow is lower. In general, the SNR of the vessel wall may be underestimated because of partial volume effects and difficulties of manually drawing a ROI around the circumferential of the vessel wall. This effect is even more obvious in the thinner basilar artery vessel wall, where the measured SNRs are consistently lower compared with the intracranial internal carotid artery vessel wall with surrounding tissue/CSF. Also, the CSF was often more suppressed 
in the pontine cistern, most likely due to a faster CSF flow, which resulted in still comparable or even higher CNRs of the basilar artery and CSF compared with the intracranial internal carotid arteries and CSF (Table 2 and Supplemental Table 1). This partly subjective determination of the circumferential of the vessel walls and CSF is subject to measurement errors. However, the same approach was used in all 7 variants and therefore it is unlikely that it influenced the relative differences in performance of the compared variants. Third, no histology or in vivo reference standard for vessel wall imaging was available of the vessel wall lesions that were identified in this study. Therefore, no validation against the ground truth could be performed. Earlier reports already described relative high numbers of vessel wall lesions or atherosclerotic plaques in asymptomatic subjects. ${ }^{28,39,40}$ In the current study mostly healthy volunteers were used for assessment of the intracranial vessel wall. Larger patient groups, including symptomatic patients, are needed for a more thorough assessment of vessel wall lesion visibility and contrastenhancement and, subsequently, the true utility of the faster vessel wall variants in practice.

\section{Conclusions}

In this study, a considerably faster clinically feasible vessel wall sequence (4 minutes 39 seconds scan duration) with high SNRs and CNRs was developed, which resulted in a good visibility of the intracranial vessel wall in the axial plane. Qualitative assessment showed promising results in overall image quality and detecting vessel wall lesions. The faster scan duration allows pre- and postcontrast acquisition of vessel wall images with sufficient remaining scan duration for other imaging sequences in patient studies. 


\section{References}

1. Dieleman N, Kolk AG, Zwanenburg JJ, Harteveld AA, Biessels GJ, Luijten PR. Imaging intracranial vessel wall pathology with magnetic resonance imaging: current prospects and future directions. Circulation 2014;130:192-201.

2. Mandell DM, Mossa-Basha M, Qiao Y, et al. Intracranial Vessel Wall MRI: Principles and Expert Consensus Recommendations of the American Society of Neuroradiology. AJNR American Journal of Neuroradiology 2017;38:218-229.

3. Qiao Y, Anwar Z, Intrapiromkul J, et al. Patterns and Implications of Intracranial Arterial Remodeling in Stroke Patients. Stroke 2016;47:434-40.

4. Zou XD, Chung YC, Zhang L, Han Y, Yang Q, Jia J. Middle Cerebral Artery Atherosclerotic Plaques in Recent Small Subcortical Infarction: A Three-Dimensional High-resolution MR Study. BioMed Research International 2015;2015:540217.

5. Yoon Y, Lee DH, Kang DW, Kwon SU, Kim JS. Single subcortical infarction and atherosclerotic plaques in the middle cerebral artery: high-resolution magnetic resonance imaging findings. Stroke 2013;44:2462-7.

6. Alexander MD, Yuan C, Rutman A, et al. High-resolution intracranial vessel wall imaging: imaging beyond the lumen. Journal of Neurology, Neurosurgery, and Psychiatry 2016;87:589-97.

7. Bodle JD, Feldmann E, Swartz RH, Rumboldt Z, Brown T, Turan TN. High-resolution magnetic resonance imaging: an emerging tool for evaluating intracranial arterial disease. Stroke 2013;44:287-92.

8. Ritz K, Denswil NP, Stam OC, van Lieshout JJ, Daemen MJ. Cause and mechanisms of intracranial atherosclerosis. Circulation 2014;130:1407-14.

9. Holmstedt CA, Turan TN, Chimowitz MI. Atherosclerotic intracranial arterial stenosis: risk factors, diagnosis, and treatment. The Lancet Neurology 2013;12:1106-14.

10. Qureshi Al, Caplan LR. Intracranial atherosclerosis. Lancet 2014;383:984-98.

11. Obusez EC, Hui F, Hajj-Ali RA, Cerejo R, Calabrese LH, Hammad T. High-resolution MRI vessel wall imaging: spatial and temporal patterns of reversible cerebral vasoconstriction syndrome and central nervous system vasculitis. AJNR American Journal of Neuroradiology 2014;35:1527-32.

12. Choi YJ, Jung SC, Lee DH. Vessel Wall Imaging of the Intracranial and Cervical Carotid Arteries. Journal of Stroke 2015;17:238-55.

13. Edjlali M, Gentric JC, Regent-Rodriguez C, Trystram D, Hassen WB, Lion S. Does aneurysmal wall enhancement on vessel wall MRI help to distinguish stable from unstable intracranial aneurysms? Stroke 2014;45:3704-6.

14. Nagahata S, Nagahata M, Obara M, et al. Wall Enhancement of the Intracranial Aneurysms Revealed by Magnetic Resonance Vessel Wall Imaging Using Three-Dimensional Turbo Spin-Echo Sequence with Motion-Sensitized Driven-Equilibrium: A Sign of Ruptured Aneurysm? Clinical Neuroradiology 2016:26:277-83. 
15. Lindenholz A, van der Kolk AG, Zwanenburg JJM, Hendrikse J. The Use and Pitfalls of Intracranial Vessel Wall Imaging: How We Do It. Radiology 2018;286:12-28.

16. Zhu $C$, Haraldsson $H$, Tian $B$, et al. High resolution imaging of the intracranial vessel wall at 3 and 7 T using 3D fast spin echo MRI. MAGMA 2016;29:559-70.

17. Wang J, Helle M, Zhou Z, Bornert P, Hatsukami TS, Yuan C. Joint blood and cerebrospinal fluid suppression for intracranial vessel wall MRI. Magnetic Resonance in Medicine 2016;75:831-8.

18. Yang H, Zhang X, Qin Q, Liu L, Wasserman BA, Qiao Y. Improved cerebrospinal fluid suppression for intracranial vessel wall MRI. Journal of magnetic resonance imaging : JMRI 2016;44:665-72.

19. Qiao Y, Steinman DA, Qin Q, et al. Intracranial arterial wall imaging using threedimensional high isotropic resolution black blood MRI at 3.0 Tesla. Journal of Magnetic Resonance Imaging : JMRI 2011;34:22-30.

20. Zhang L, Zhang N, Wu J, Huang Y, Liu X, Chung YC. High resolution three dimensional intracranial arterial wall imaging at $3 \mathrm{~T}$ using T1 weighted SPACE. Magnetic Resonance Imaging 2015;33:1026-34.

21. Xie Y, Yang Q, Xie G, Pang J, Fan Z, Li D. Improved black-blood imaging using DANTESPACE for simultaneous carotid and intracranial vessel wall evaluation. Magnetic Resonance in Medicine 2016;75:2286-94.

22. Li ML, Xu YY, Hou B, et al. High-resolution intracranial vessel wall imaging using 3D CUBE T1 weighted sequence. European Journal of Radiology 2016;85:803-7.

23. Dieleman N, Yang W, van der Kolk AG, et al. Qualitative Evaluation of a High-Resolution 3D Multi-Sequence Intracranial Vessel Wall Protocol at 3 Tesla MRI. PloS one 2016;11:e0160781.

24. Dieleman N, Yang W, Abrigo JM, et al. Magnetic Resonance Imaging of Plaque Morphology, Burden, and Distribution in Patients With Symptomatic Middle Cerebral Artery Stenosis. Stroke 2016; 47:1797-1802.

25. Klein S, Staring M, Murphy K, Viergever MA, Pluim JP. elastix: a toolbox for intensity-based medical image registration. IEEE Transactions on Medical Imaging 2010;29:196-205.

26. Swartz RH, Bhuta SS, Farb RI, et al. Intracranial arterial wall imaging using high-resolution 3-tesla contrast-enhanced MRI. Neurology 2009;72:627-34.

27. Fan Z, Yang Q, Deng Z, et al. Whole-brain intracranial vessel wall imaging at 3 Tesla using cerebrospinal fluid-attenuated T1-weighted 3D turbo spin echo. Magnetic Resonance in Medicine 2017:77:1142-1150.

28. Harteveld AA, van der Kolk AG, van der Worp HB, et al. High-resolution intracranial vessel wall MRI in an elderly asymptomatic population: comparison of 3T and 7T. European Radiology 2017;27:1585-1595.

29. Li L, Miller KL, Jezzard P. DANTE-prepared pulse trains: a novel approach to motionsensitized and motion-suppressed quantitative magnetic resonance imaging. Magnetic Resonance in Medicine 2012;68:1423-38. 
30. Edelman RR, Chien D, Kim D. Fast selective black blood MR imaging. Radiology 1991;181:655-60.

31. van der Kolk AG, Hendrikse J, Brundel M, Biessels GJ, Smit EJ, Visser F. Multi-sequence whole-brain intracranial vessel wall imaging at 7.0 tesla. European Radiology 2013;23:2996-3004.

32. Mugler JP, 3rd. Optimized three-dimensional fast-spin-echo MRI. Journal of Magnetic Resonance Imaging : JMRI 2014;39:745-67.

33. Dieleman N, van der Kolk AG, van Veluw SJ, et al. Patterns of intracranial vessel wall changes in relation to ischemic infarcts. Neurology 2014;83:1316-20.

34. Antiga L, Wasserman BA, Steinman DA. On the overestimation of early wall thickening at the carotid bulb by black blood MRI, with implications for coronary and vulnerable plaque imaging. Magnetic Resonance in Medicine 2008;60.

35. Xu W-H, Li M-L, Gao S, Ni J, Zhou L-X, Yao M. In vivo high-resolution MR imaging of symptomatic and asymptomatic middle cerebral artery atherosclerotic stenosis. Atherosclerosis 2010;212:507-11.

36. Wiesinger F, Van de Moortele PF, Adriany G, De Zanche N, Ugurbil K, Pruessmann KP. Potential and feasibility of parallel MRI at high field. NMR in biomedicine 2006;19:36878.

37. Lustig M, Donoho D, Pauly JM. Sparse MRI: The application of compressed sensing for rapid MR imaging. Magnetic Resonance in Medicine 2007;58:1182-95.

38. Li B, Li H, Li J, et al. Relaxation enhanced compressed sensing three-dimensional black-blood vessel wall MR imaging: Preliminary studies. Magnetic Resonance Imaging 2015;33:932-8.

39. Bos D, van der Rijk MJ, Geeraedts TE, et al. Intracranial carotid artery atherosclerosis: prevalence and risk factors in the general population. Stroke 2012;43:1878-84.

40. Homburg PJ, Plas GJ, Rozie S, van der Lugt A, Dippel DW. Prevalence and calcification of intracranial arterial stenotic lesions as assessed with multidetector computed tomography angiography. Stroke 2011;42:1244- 


\section{Supplemental Material}

Supplemental Table 1. SNR and CNR of the different (precontrast-only) scan variants

\begin{tabular}{|c|c|c|c|c|c|c|c|}
\hline & $\begin{array}{l}\mathbf{T}_{1 \mathrm{w}} \text { VISTA } \\
\text { variant } 1\end{array}$ & $\begin{array}{l}\text { PD }_{\mathrm{w}} \text { VISTA } \\
\text { variant } 2^{\mathrm{E}}\end{array}$ & $\begin{array}{l}\mathbf{T}_{1 \mathrm{w}} \text { VIRTA } \\
\text { variant } 3^{\mathrm{F}}\end{array}$ & $\begin{array}{l}\text { T }_{1 \mathrm{~W}} \text { VIRTA } \\
\text { variant } 4\end{array}$ & $\begin{array}{c}\text { T }_{1 \mathrm{w}} \text { VISTA } \\
\text { variant } 5^{\mathrm{G}}\end{array}$ & $\begin{array}{l}\mathrm{T}_{1 \mathrm{w}} \text { VISTA } \\
\text { variant } 6\end{array}$ & $\begin{array}{l}\mathrm{T}_{1 \mathrm{w}} \text { VIRTA } \\
\text { variant } 7\end{array}$ \\
\hline SNR $_{\text {tissue }}{ }^{A}$ & $15.8 \pm 0.9$ & $31.8 \pm 2.5$ & $27.9 \pm 1.8$ & $43.4 \pm 3.4$ & $25.5 \pm 2.2$ & $21.2 \pm 1.6$ & $45.3 \pm 3.6$ \\
\hline SNR $_{\text {carotid vessel wall }}{ }^{B}$ & $8.2 \pm 0.4$ & $21 \pm 1.8$ & $14 \pm 0.5$ & $27.3 \pm 2.1$ & $16.1 \pm 1.8$ & $10.4 \pm 0.4$ & $21.4 \pm 2.8$ \\
\hline SNR $_{\text {basilary vessel wall }}$ & $6.1 \pm 0.6$ & $15.3 \pm 2.9$ & $11 \pm 1.9$ & $21.2 \pm 5.9$ & $11.8 \pm 1.9$ & $7.9 \pm 0.7$ & $15.2 \pm 2.8$ \\
\hline SNR $_{\text {suprasellar CSF }}$ & $6.9 \pm 0.5$ & $17.8 \pm 3.2$ & $12.2 \pm 0.8$ & $24.3 \pm 1.1$ & $13.8 \pm 0.9$ & $8.7 \pm 1.0$ & $16.6 \pm 2.1$ \\
\hline SNR $_{\text {pontine CSF }}$ & $2.5 \pm 0.4$ & $10 \pm 3.9$ & $5.9 \pm 1.9$ & $12.8 \pm 5.4$ & $7.7 \pm 2.7$ & $3.7 \pm 1.1$ & $10.8 \pm 2.6$ \\
\hline SNR $_{\text {blood }}$ & $2.5 \pm 0.2$ & $4.4 \pm 0.8$ & $3.4 \pm 0.4$ & $4.2 \pm 1.1$ & $3.3 \pm 0.8$ & $2.5 \pm 0.7$ & $4.7 \pm 1.1$ \\
\hline $\mathrm{CNR}_{\text {carotid vessel wall-CSF }}{ }^{\mathrm{C}}$ & $1.3 \pm 0.6$ & $3.2 \pm 2.3$ & $1.8 \pm 0.4$ & $3.0 \pm 1.9$ & $2.2 \pm 1.1$ & $1.7 \pm 1.2$ & $4.8 \pm 1.5$ \\
\hline CNR $_{\text {basilary vessel wall-CSF }}{ }^{D}$ & $3.6 \pm 0.4$ & $5.3 \pm 1.7$ & $5.1 \pm 2.0$ & $8.4 \pm 3.9$ & $5.2 \pm 0.9$ & $4.1 \pm 1.5$ & $4.4 \pm 1.6$ \\
\hline $\begin{array}{l}\text { CNR } \text { carotid vessel wall - }_{\text {- }} \text { blood }\end{array}$ & $5.7 \pm 0.4$ & $16.6 \pm 0.9$ & $10.6 \pm 0.8$ & $23.1 \pm 2.7$ & $12.7 \pm 1.5$ & $8 \pm 0.9$ & $16.7 \pm 2.5$ \\
\hline
\end{tabular}

Means and standard deviations (expressed as mean \pm SD) are calculated over 5 subjects (precontrast). A. Tissue region of interest (ROI) is located at the left orbital gyri. B. The mean of the left and right distal intracranial internal carotid artery. C. Suprasellar CSF is used as reference. D. Pontine CSF is used as reference. E. Adjusted from Qiao et al. ${ }^{19} \mathbf{F}$. Currently used in our clinic G. Adjusted from Qiao et al. ${ }^{19}$ with a shorter TR. VISTA = volumetric isotropic turbo spin echo acquisition, VIRTA = volumetric isotropically reconstructed turbo spin echo acquisition, $\mathrm{PD}$ = proton density, SNR = signal-to-noise ratio, CNR = contrast-to-noise ratio, CSF = cerebrospinal fluid. 


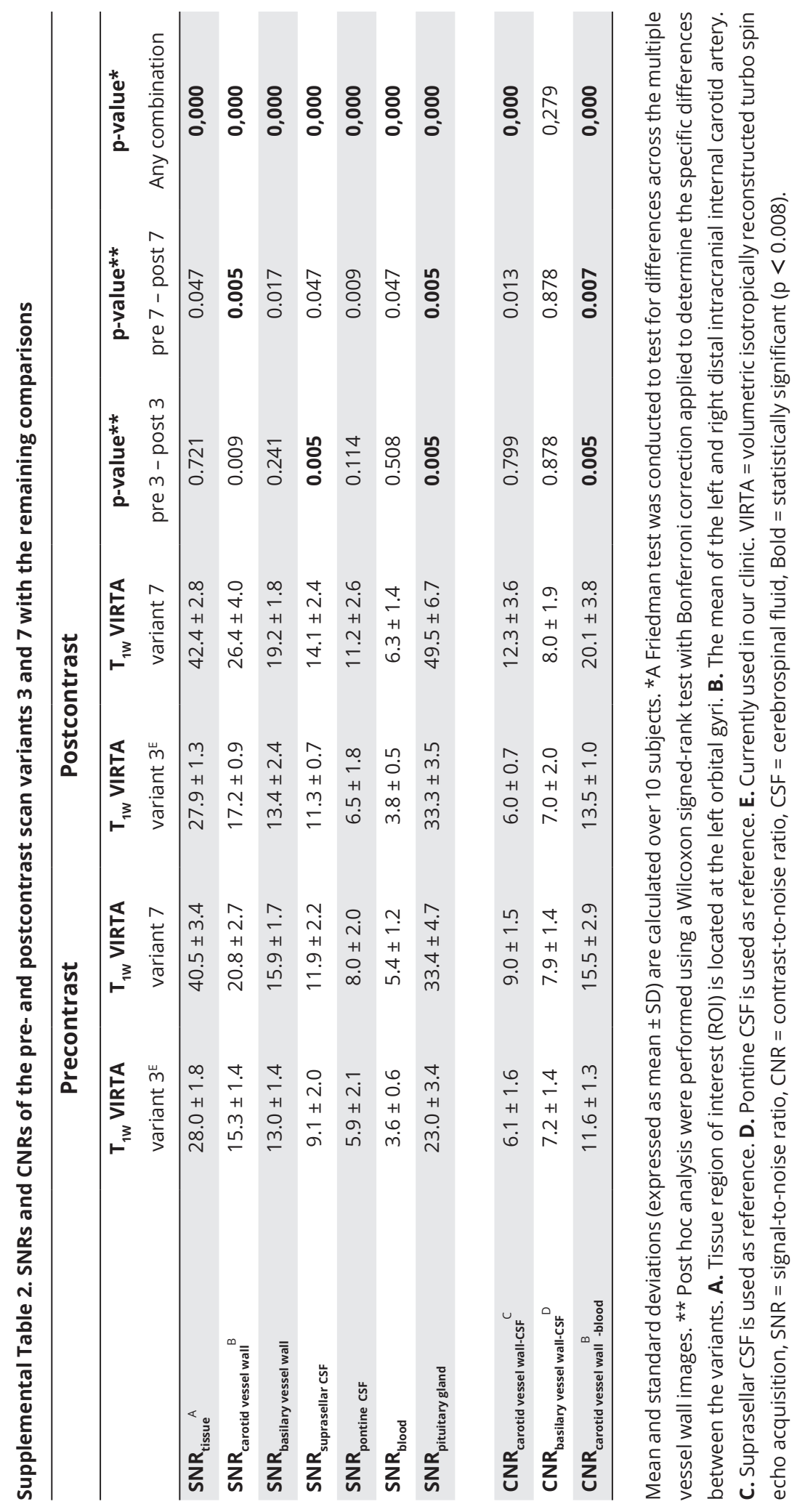


Comparison of 3T Intracranial Vessel Wall MRI Sequences

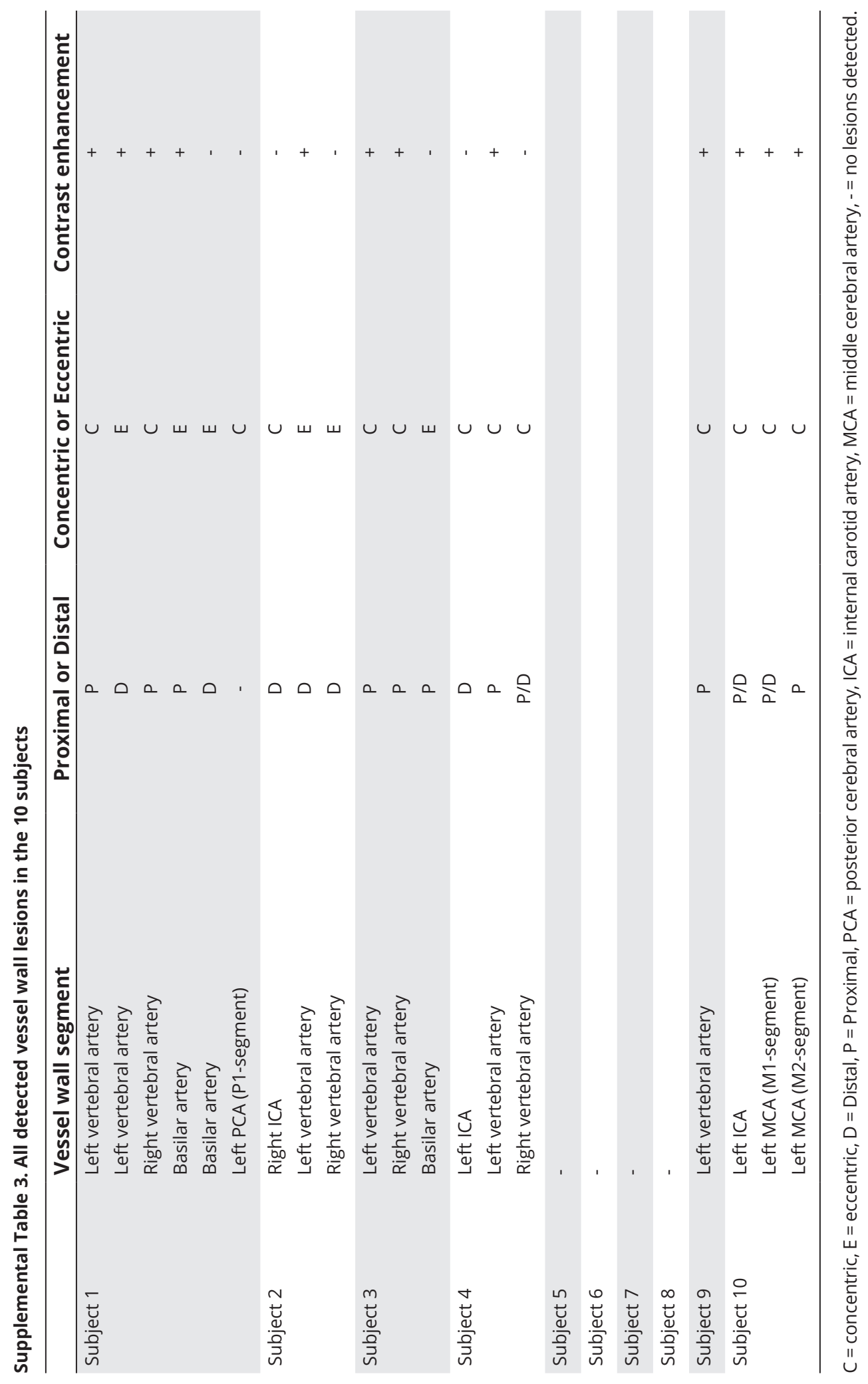




\section{Supplemental Figures}

\section{Supplemental Figure 1}
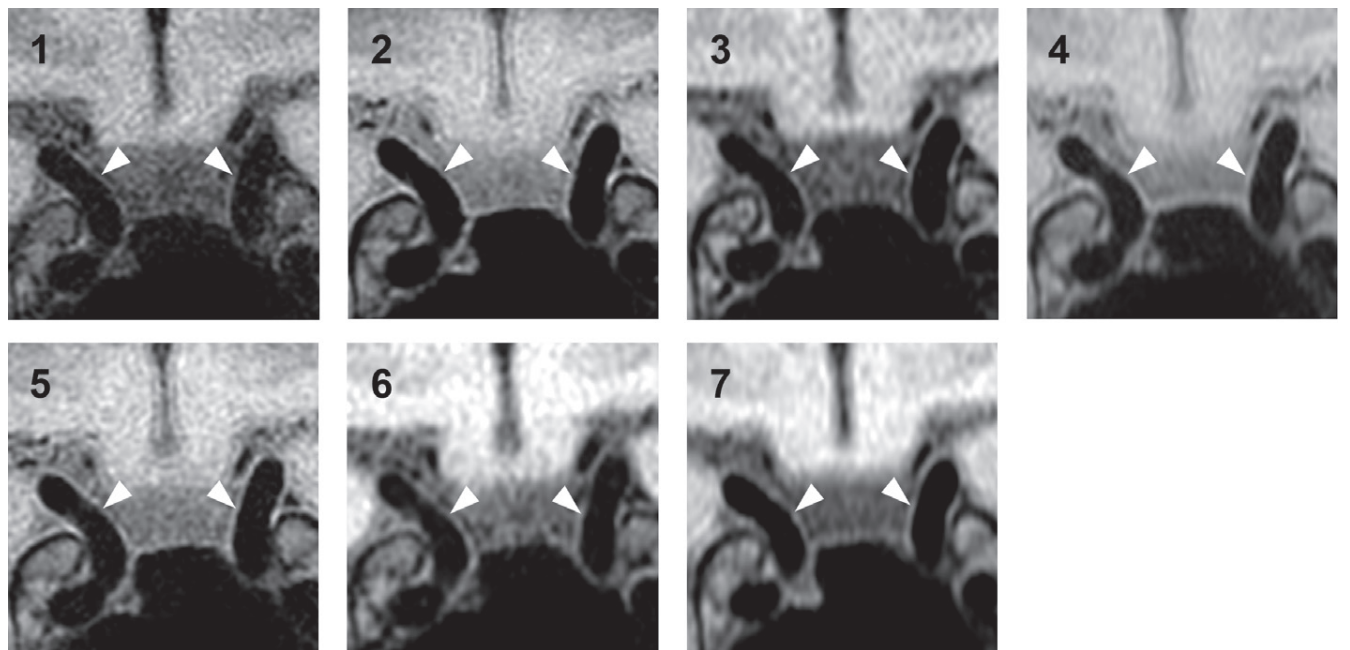

Supplemental Figure 1. Example images in coronal plane of the 7 different scan variants that were performed at $3 \mathrm{~T}$ (precontrast; in order of decreasing scan duration). Both distal intracranial internal carotid arteries (white arrowheads) are depicted in all images, surrounded by CSF and brain parenchyma. 1. $T_{1 W}$ VISTA variant 1 (8:24min). 2. PD ${ }_{W}$ VISTA variant 2 (7:50 min), adjusted from Qiao et al. ${ }^{19}$ 3. $T_{1 W}$ VIRTA variant 3 (6:42min). 4. $T_{1 \mathrm{~W}}$ VIRTA variant 4 (6.01 min). 5. $T_{1 \mathrm{~W}}$ VISTA variant 5 (5:52 min), adjusted from Qiao et al. with a shorter TR. ${ }^{19}$ 6. $T_{1 \mathrm{~W}}$ VISTA variant 6 (5:49min). 7. $\mathrm{T}_{1 \mathrm{~W}}$ VIRTA variant 7 (4:39min). VISTA = volumetric isotropic turbo-spin-echo acquisition, VIRTA = volumetric isotropically reconstructed turbo-spin echo-acquisition 
Supplemental Figure 2

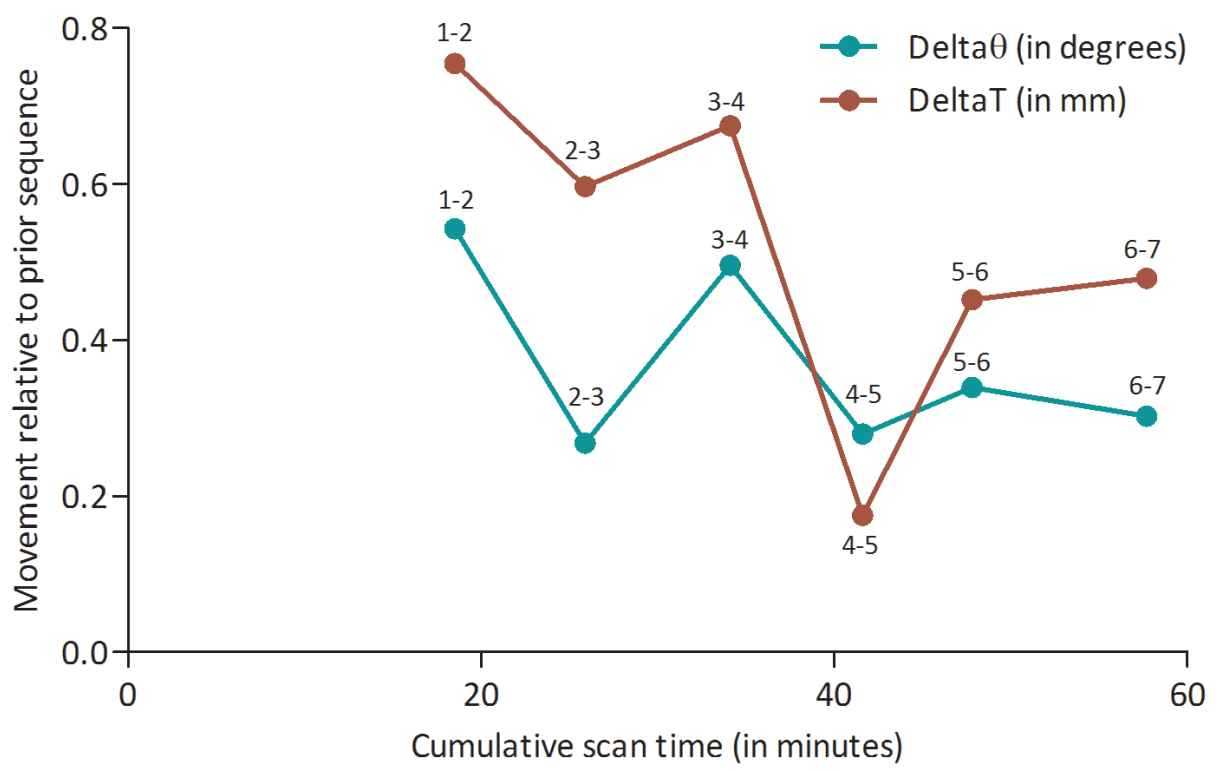

Supplemental Figure 2. The degree of movement during acquisition time expressed as $\Delta$ Rotation (in degrees) and $\Delta$ Translation (in $\mathrm{mm}$ ) between all consecutive acquired vessel wall sequences. Each vessel wall sequence is compared with the prior acquired sequence according to the scan protocol (consecutively variant 2, 5, 3, 4, 7, 1 and 6; Table 1). The mean registration parameters are calculated over 5 subjects. The $\Delta$ Rotation and $\Delta$ Translation parameters were calculated as $\sqrt{ }\left(\mathrm{X}\right.$-axis ${ }^{2}+\mathrm{Y}$-axis ${ }^{2}+\mathrm{Z}$-axis $\left.{ }^{2}\right)$. Over time, there was no increase in motion in the later scheduled sequences. 


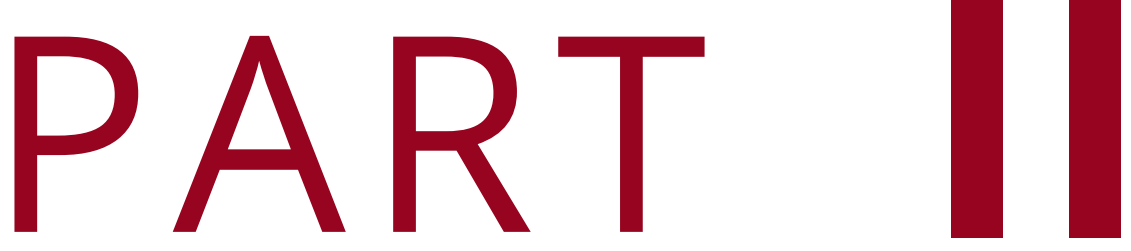

Clinical Translation 


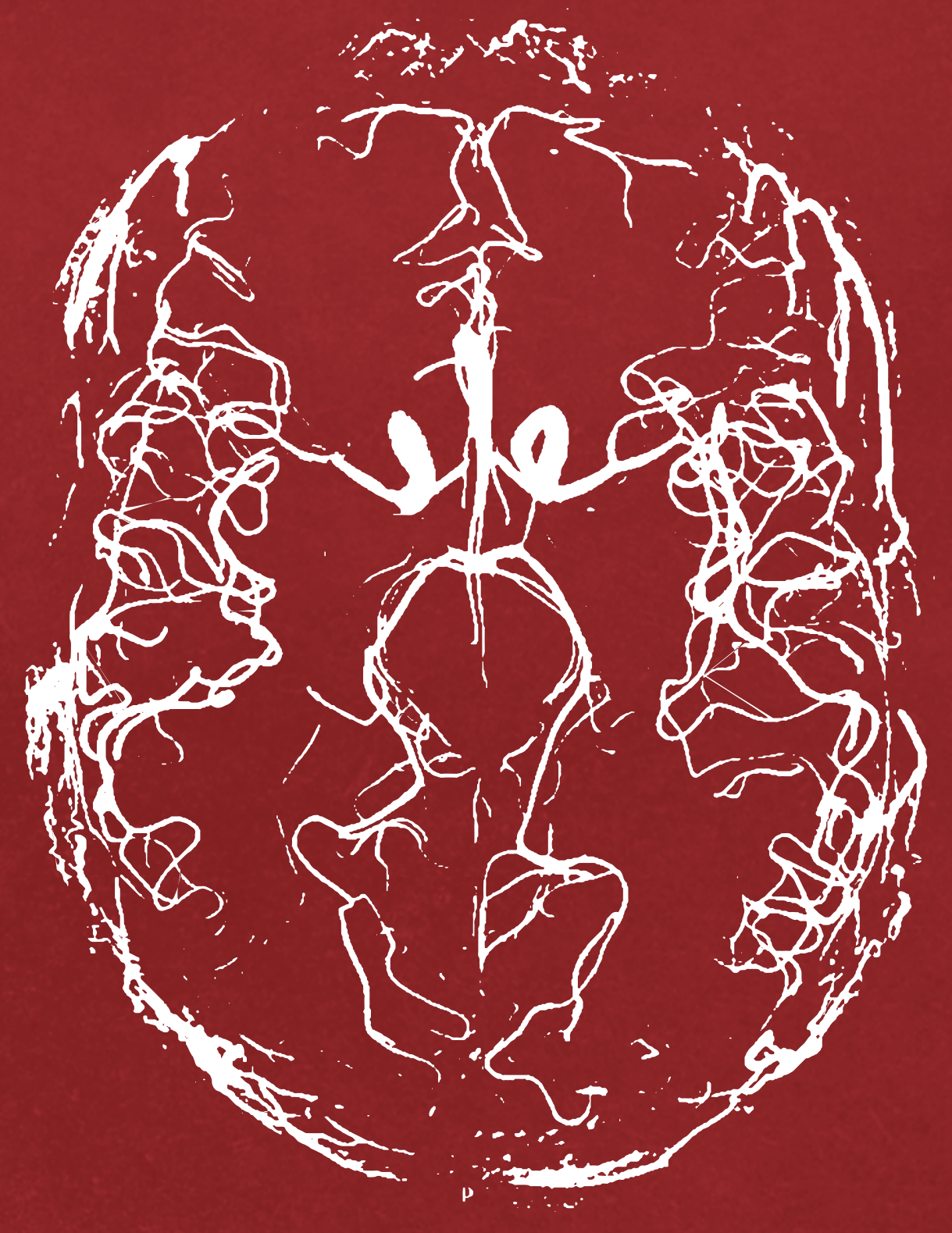




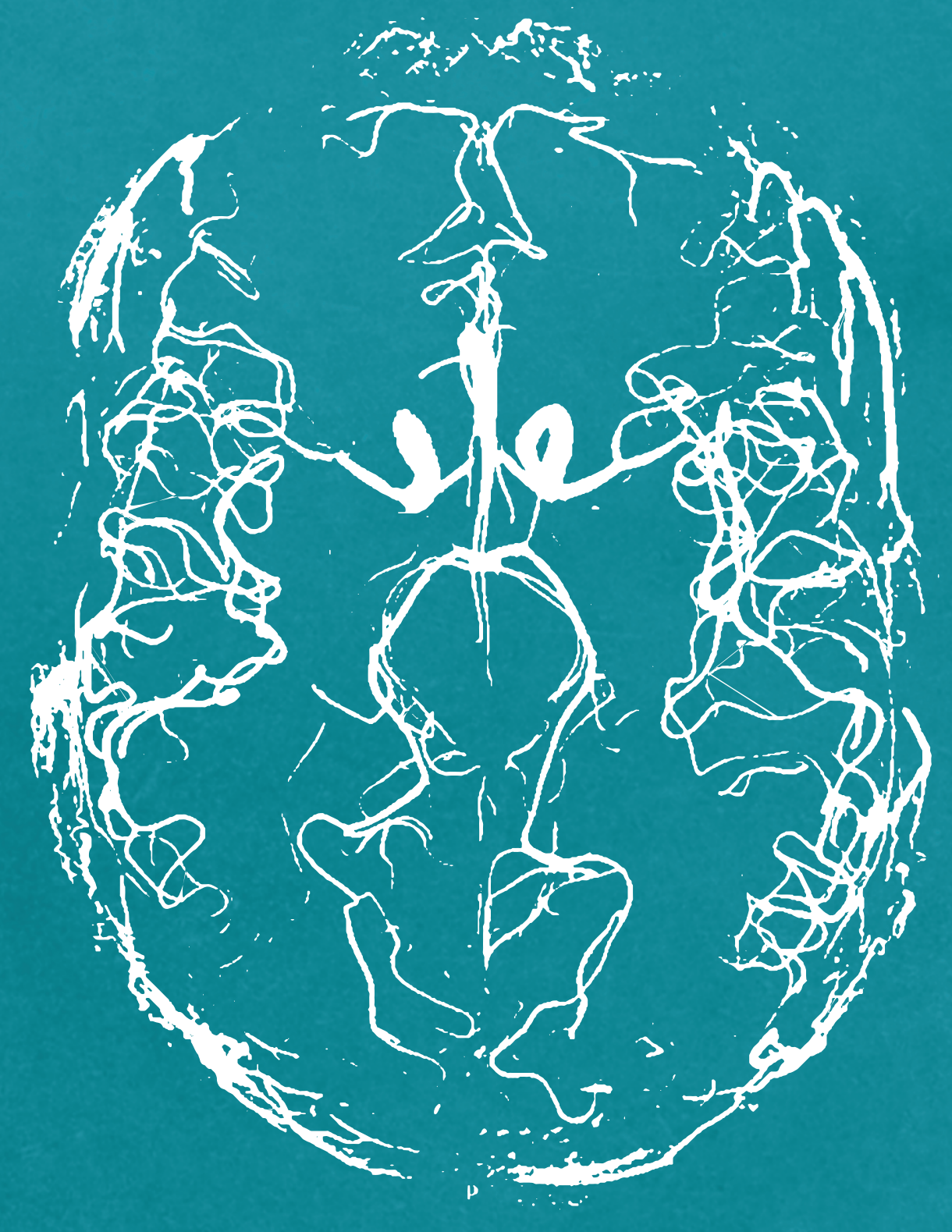




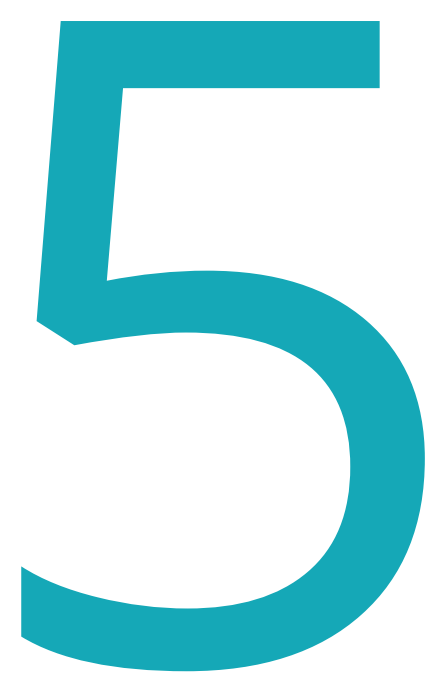

\section{Intracranial Atherosclerosis Assessed with 7T MRI: Evaluation of Patients with Ischemic Stroke or Transient Ischemic Attack}

Arjen Lindenholz', Anja G. van der Kolk', Irene C. van der Schaaf', H. Bart van der Worp' ${ }^{2}$, Anita A. Harteveld', Nikki Dieleman', Michiel L. Bots ${ }^{3}$ and Jeroen Hendrikse $^{1}$

${ }^{1}$ Department of Radiology, University Medical Center Utrecht, The Netherlands ${ }^{2}$ Department of Neurology and Neurosurgery, University Medical Center Utrecht, Brain Center Rudolf Magnus, The Netherlands ${ }^{3}$ Julius Center for Health Sciences and Primary Care, University Medical Center Utrecht, The Netherlands 


\begin{abstract}
Background: Intracranial atherosclerosis is an important cause of ischemic stroke and is associated with several vascular risk factors. Current imaging is mainly based on the assessment of luminal narrowing rather than abnormalities in the vessel wall.
\end{abstract}

Purpose: To investigate the relation between vascular risk factors and atherosclerotic lesion burden of intracranial arteries assessed with vessel wall MRI at 7T in participants with ischemic stroke or transient ischemic attack (TIA).

Materials and Methods: In this prospective study (Trial ID NTR2119, www.trialregister.nl), study participants who presented with ischemic stroke or TIA of the anterior circulation between December 2009 and September 2017 underwent pre- and postcontrast 7T vessel wall MRI within three months onset. All large arteries of the intracranial circulation were assessed for number, location, and enhancement of vessel wall lesions. Generalized Estimating Equations for Poisson regression were used to investigate the relationship between vascular risk factors and number or enhancement of vessel wall lesions.

Results: Ninety participants (52 men; mean age, 60 years) were evaluated. Increasing age (relative risk (RR), 1.02; 95\% confidence interval $(\mathrm{Cl}), 1.01-1.03)$, hypertension (RR, 1.46; 95\% Cl, 1.06-2.02), diabetes mellitus (RR, 1.67; 95\% Cl, 1.20 2.33), and a higher multivariable vascular risk score (the Second Manifestations of ARTerial disease (SMART) risk score, RR, 1.01; 95\% Cl, 1.00-1.02) were associated with a higher number of vessel wall lesions in the anterior circulation. Contrastenhancing vessel wall lesions were only associated with increasing age (RR, 1.03; $95 \% \mathrm{Cl}, 1.01-1.05)$. No association was found between smoking and the number of vessel wall lesions.

Conclusions: Except for smoking, traditional common cardiovascular risk factors were associated with a higher number and enhancement of intracranial vessel wall lesions at 7T MRI in individuals evaluated after ischemic stroke or transient ischemic attack. 


\section{Introduction}

Intracranial atherosclerosis (ICAS) is an important cause of ischemic stroke, ${ }^{1}$ especially in black, Hispanic, and Asian populations, where ICAS accounts for up to $50 \%$ of all ischemic stroke or transient ischemic attacks (TIA) ${ }^{1-4}$ In white populations, ICAS accounts for $5 \%-23 \%$ of ischemic strokes..$^{2,5,6}$ ICAS has been associated with many of the risk factors for atherosclerotic disease, including hypertension, hyperlipidemia, diabetes mellitus, and smoking. ${ }^{2-4,7-10}$ However, those studies used lumenographic techniques (e.g., CT angiography, MR angiography, or intra-arterial digital subtraction angiography) to assess intracranial calcifications or stenosis as a proxy for ICAS. ${ }^{3-5,7,8}$ Although these methods enable the accurate assessment of luminal narrowing, they are limited in assessing the vessel wall itself. ${ }^{11,12}$ Because of arterial remodeling, ICAS results in luminal narrowing only at a more advanced stage of the disease.13,14 Therefore, subtle atherosclerotic plaques or other nonstenotic intracranial vessel wall lesions are easily missed with these lumenographic techniques.

With high-field (3 tesla (T) and 7T) intracranial MRI, both stenotic and nonstenotic vessel wall lesions can be detected. ${ }^{15-18}$ Specifically developed intracranial vessel wall sequences have therefore been increasingly used to evaluate intracranial atherosclerotic disease, including the characterization of vessel wall lesions and possible contrast enhancement of the vessel wall. ${ }^{15,19}$ For the first time, to our knowledge, a recent American study used intracranial vessel wall MRI at $3 T$ to assess the relationship between vascular risk factors and intracranial atherosclerosis. ${ }^{20}$ However, this study was performed in a community-based cohort and its main focus was on the role of ethnicity - not TIA or stroke - on ICAS prevalence. We hypothesize that increasing age, hypertension, male sex and hyperlipidemia increase the vessel wall lesion burden in Caucasian participants with an ischemic stroke or TIA. In our study, we investigated the relation between vascular risk factors and intracranial vessel wall lesion burden using intracranial vessel wall MRI at 7T in participants with ischemic stroke or TIA. 


\section{Materials and Methods}

\section{Study participants}

From December 2009 to September 2017, patients who presented in our University Medical Center Utrecht with ischemic stroke or TIA of the anterior circulation could be included in the intracranial vessel wall imaging (IVI) study (Trial ID NTR2119, www. trialregisters.nl). Details of this study have been reported previously. ${ }^{16,21}$ Additional inclusion criteria were age older than 18 years, the possibility of undergoing 7T MRI within 3 months after symptom onset, and sufficient understanding of the Dutch language to provide written informed consent. Participants were excluded when TIA or ischemic stroke was secondary to a surgical or radiological intervention, when renal function was impaired (estimated glomerular filtration rate $<30 \mathrm{~mL} /$ $\min / 1,73 \mathrm{~m}^{2}$ ), or if there was contraindication to $7 \mathrm{~T}$ MRI (claustrophobia, contraindicated metal objects in or on the body, pregnancy, or physically unable to endure an MRI examination) or to gadolinium-containing contrast agents. A flowchart of the study inclusion is shown in Figure 1. This prospective study was approved by the institutional review board of our hospital, and all participants gave written informed consent.

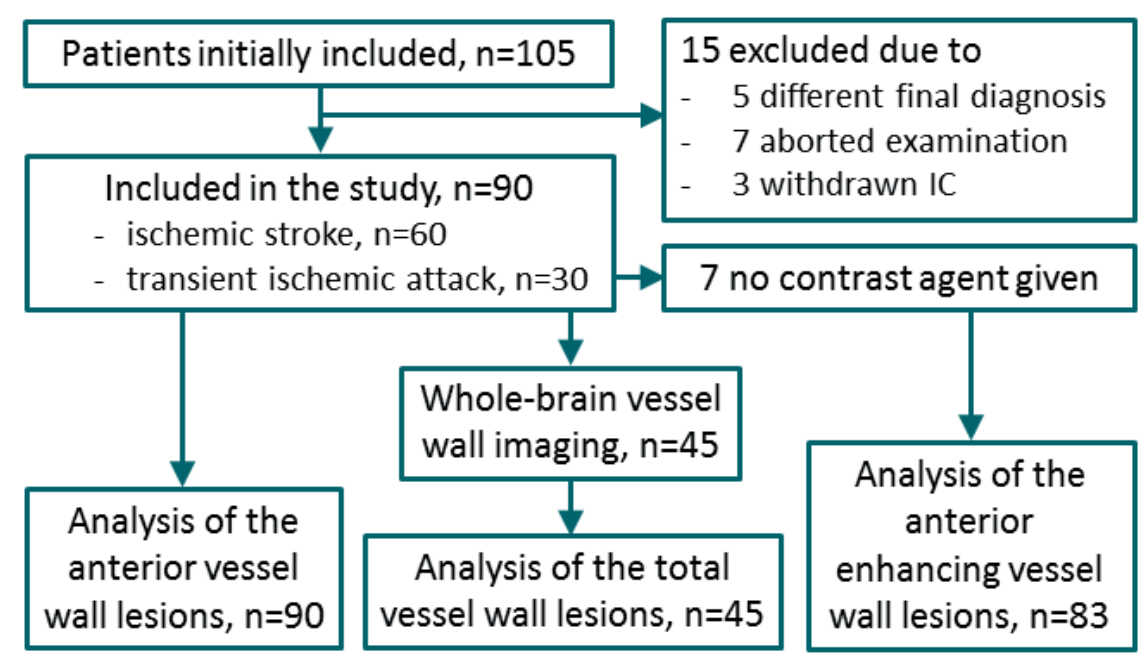

Figure 1. Flowchart shows the study inclusion and exclusion criteria with the number of participants that were included for analysis. IC, informed consent.

\section{MRI protocol}

MRI was performed on a 7T whole-body system (Philips Healthcare, Cleveland, Ohio) with either a 16-channel (Nova Medical, Wilmington, MA) $(n=40)$ or because of hardware updates during the study inclusion period - a 32-channel ( $n=$ 50) receive coil, and a volume transmit-receive coil for transmission (Nova Medical, 
Wilmington, MA). The MRI protocol included a pre- and postcontrast threedimensional (3D) $\mathrm{T}_{1}$-weighted intracranial vessel wall sequence (magnetizationprepared inversion recovery turbo spin echo, MPIR-TSE), diffusion weighted imaging (DWI) sequence, $T_{2}$-weighted fluid-attenuated inversion recovery (FLAIR) sequence and a time-of-flight MR angiography (TOF-MRA) sequence. Postcontrast MRI was acquired 5-10 minutes after precontrast MRI. A gadolinium-containing contrast agent (gadobutrol, Gadovist $1.0 \mathrm{mmol} / \mathrm{mL}$, Bayer Schering Pharma, Newbury, UK), with dose adjusted to participant weight, was administered intravenously an average 5 minutes before the performance of the postcontrast MPIR-TSE sequence. The following imaging parameters were used for the 3D MPIRTSE sequence: sagittal acquisition, field-of-view (FOV), $220 \times 180 \times 13 \mathrm{~mm}(n=45)$, which was the single scan parameter that was optimized during the study period to $250 \times 250 \times 190 \mathrm{~mm}$ (whole brain; $n=45$ ) following technical developments, acquired spatial resolution, $0.8 \times 0.8 \times 0.8 \mathrm{~mm}^{3}$; reconstructed spatial resolution, $0.5 \times 0.5 \times 0.5 \mathrm{~mm}^{3}$; repetition time (TR), $3952 \mathrm{~ms}$; echo time (TE), $37 \mathrm{~ms}$; inversion time (TI), 1375 ms; and acquisition time 10 minutes 40 seconds. ${ }^{16,18,21}$

For the initial 3D MPIR-TSE sequence, the smaller FOV was planned in such a way (transverse / oblique) that the large cerebral arteries of the circle of Willis were within the imaging plane, which included the distal intracranial carotid arteries, the anterior, middle and posterior cerebral arteries, and whenever possible, the basilar artery and vertebral arteries. The two 3D MPIR-TSE sequences with small and large FOV were tested for equality during the study inclusion. ${ }^{21}$ Imaging parameters of the TOF-MRA included: FOV, $190 \times 190 \times 102 \mathrm{~mm}$; acquired spatial resolution, $0.4 \times 0.5 \times 0.6 \mathrm{~mm}^{3}$; reconstructed spatial resolution, $0.4 \times 0.4 \times 0.3 \mathrm{~mm}^{3}$; TR, $21 \mathrm{~ms}$; TE, $2.3 \mathrm{~ms}$; and acquisition time 9 minutes 18 seconds. ${ }^{18}$ The images from the TOF-MRA were used for anatomical verification of the vessels seen on the MPIR-TSE images.

\section{Data collection}

For all participants, demographic information, vascular risk factors, ${ }^{8}$ final clinical diagnosis and the Trial of ORG 10172 in Acute Stroke Treatment, or TOAST, classification for classifying stroke/TIA subtype were recorded.22 The vascular risk factors included: body mass index (BMI), hypertension (yes or no), systolic and diastolic blood pressure, hyperlipidemia (yes or no; including low-density lipoprotein (LDL) cholesterol, high-density lipoprotein (HDL) cholesterol, and triglycerides (all in $\mathrm{mmol} / \mathrm{L}$ )), diabetes mellitus (yes or no), any peripheral artery disease (yes or no), any previous cardiovascular disease (yes or no; e.g., angina pectoris, atrial fibrillation, myocardial infarction), renal function (expressed in estimated glomerular filtration rate (eGFR), calculated with the Modification of Diet in Renal Disease (MDRD) equation), C-reactive protein (CRP) level, and smoking status (never, current or former smoking, calculated in pack years). 
Hypertension was defined as systolic blood pressure greater than $140 \mathrm{mmHg}$ or diastolic blood pressure greater than $90 \mathrm{mmHg}$ or use of antihypertensive drugs; ${ }^{23}$ diabetes mellitus as fasting serum glucose levels greater than $6.9 \mathrm{mmol} / \mathrm{L}$ or use of antidiabetic drugs; ${ }^{24}$ and hyperlipidemia as either total serum cholesterol level greater than $6.2 \mathrm{mmol} / \mathrm{L}$, LDL cholesterol level greater than $4.1 \mathrm{mmol} / \mathrm{L}$, HDL cholesterol level less than $1.0 \mathrm{mmol} / \mathrm{L}$, triglyceride level greater than $2.2 \mathrm{mmol} / \mathrm{L}$, or use of lipid-lowering drugs. ${ }^{25}$ From the collected data, the Second Manifestations of ARTerial disease (SMART) vascular risk score was calculated to combine risk factors (age, sex, current smoking status, systolic blood pressure, diabetes mellitus, coronary artery disease, cerebrovascular disease, abdominal aortic aneurysm, peripheral artery disease, time since first cardiovascular diagnose, $\mathrm{HDL}$ cholesterol, total cholesterol, eGFR, and high-sensitivity (RP) into one score. ${ }^{26}$ This score predicts the 10-year risk of recurrent vascular events in participants with clinically manifest atherosclerosis.

\section{Data evaluation and MRI analysis}

All MRI scans were assessed with an offline workstation (Philips DICOM Viewer 3.0, Philips Healthcare, Best, Netherlands). Two readers (AK, 8 years of experience and AL, 3 years of experience) independently assessed the MRI series of all participants $(n=90)$. Readers were blinded for any patient characteristics. The degree of reliability and agreement was calculated and compared with the levels of agreement published in recent literature..$^{27,28}$ In addition, after 15 image assessments, the two readers held an interim consensus meeting as quality assurance for lesiondefinition adherence and to improve the accuracy and consistency of vessel wall assessment for the whole dataset. Any discrepancies were resolved, and, in the case of disagreement, a third reader $\mathrm{JH}$, neuroradiologist with $>10$ years of experience) was consulted. The count results of the most trained reader (AK, 8 years of experience) were used for the descriptive results (Supplemental Table 1). Arterial vessel segments that were assessed bilaterally for vessel wall lesions included the distal internal carotid artery (ICA, clinoid, supra-clinoid and terminal segments), middle cerebral artery (MCA; M1 and M2 segments), proximal anterior cerebral artery (ACA; $A 1$ and $A 2$ segments), posterior cerebral artery (PCA; P1 and $P 2$ segments), intracranial segment of the vertebral artery (VA), and the basilar artery (BA). In general, the process of lesion detection, with the comparison with the normal-appearing vessel segments either on the ipsilateral and contralateral side and possible lesion enhancement is in agreement with the radiology practice of interpreting intracranial vessel wall lesions in our radiology department, as has been described in more detail in a recent publication in Radiology.$^{18} \mathrm{~A}$ vessel wall lesion was defined as a visually defined focal or diffuse thickening of the vessel wall greater than $50 \%$ compared with the adjacent or contralateral vessel wall thickness on both pre- and postcontrast images. The vessel wall images could be 
assessed in multiple planes because of the isotropic voxel size. When the vessel wall regions were bilaterally affected, contralateral assessment was performed with extra caution or was omitted. For assessment of contrast enhancement, postcontrast vessel wall MRI scans were assessed for higher signal intensity over at least two slices using the corresponding precontrast MRI scan as a reference. In addition, pre- and postcontrast vessel wall MRI scans were coregistered and subsequently matched for the whole 3D volume using advanced algorithms of the elastix toolbox in MeVisLab (version 2.7; MeVis Medical Solutions, Bremen, Germany). ${ }^{29}$ The precontrast images were subtracted from the coregistered postcontrast images, and the resulting subtraction images were then used to visually confirm contrast enhancement. ${ }^{18}$ Vessel wall enhancement at the location where the ICA or the vertebral artery pierces the dura mater, which is suspicious for vasa vasorum extension in the intracranial portions of the vessel wall, was not counted as an enhancing vessel wall lesion. ${ }^{18,30}$

For participants who underwent imaging during the first 1.5 years of the study with the smaller FOV MPIR-TSE sequence, the posterior circulation could not be completely assessed for vessel wall lesions because the vertebral arteries and basilar artery were often outside the FOV. Therefore, we differentiated between anterior and posterior circulation lesion burden, with lesions in the anterior circulation as the primary outcome. In participants in whom both the anterior and posterior circulation could be assessed, an additional analysis was performed with the total burden of vessel wall lesions as outcome.

\section{Statistical analysis}

The intraclass correlation coefficient (ICC) (with a two-way mixed, single measurements, consistency model for the number of detected vessel wall lesions) and the Dice similarity coefficient (DSC) (for the number of vessel wall lesions detected at the same location) were calculated to measure interrater reproducibility. ${ }^{31} \mathrm{~A}$ Bland-Altman plot was used to assess the agreement between the two readers. Descriptive statistics are given in percentages or are summarized as means with a range or standard deviation. Generalized Estimating Equations (GEE) for Poisson regression were performed to assess the relation between vascular risk factors and the number of (enhancing) vessel wall lesions in the anterior circulation. We selected a maximum of 10 predictors, apart from age and sex, to avoid overfitting (one-in-10 rule) which were previously described as wellestablished risk factors for ICAS to include in the GEE Poisson regression models. ${ }^{8}$ All included variables were tested for multicollinearity using both Pearson and Spearman correlation methods and assessed for the Goodness-of-Fit by using R and $\mathrm{R}^{2}$. Where possible, we used continuous variables over dichotomous variables in the regression analysis for improving statistical reliability. ${ }^{32}$ The vascular risk 
score (SMART risk score) was calculated for each participant to act as a proxy for a multivariable analysis, which included 14 variables. Age and sex were included as covariates. The GEE Poisson regression models, with a robust variance estimator, were corrected for overdispersion using the Pearson chi-square method. For the additional analysis of the total burden of vessel wall lesions in the anterior and posterior circulation, the same method was used. Poisson regression generally provides rate ratios, which are interpretable as relative risks (RRs). Associations are therefore presented in more commonly used RRs with 95\% confidence intervals (Cls). A Bonferroni correction for multiple testing was performed, and a two-sided p-value of 0.0013 (0.05 divided by 39 ) was indicative of a statistically significant difference. A two-sided $p$-value $<0.05$ was considered to indicate trend. Statistical analyses were performed using SPSS version 21.0 (IBM SPSS Statistics, IBM Corp., Armonk, NY).

\section{Results}

\section{Participants characteristics}

Participant characteristics are summarized in Table 1. Of the 105 patients included in this study, 90 met the inclusion criteria. Fifteen patients were excluded because of a final diagnosis other than TIA or ischemic stroke $(n=5)$, aborted MRI examination due to claustrophobia or participant discomfort $(n=7)$, or withdrawn informed consent $(n=3)$ (Figure 1). Of the 90 study participants, the average age was 60 years (range 27-85 years) and 52 were man (58\%). Forty-six of the 90 participants had hypertension, 44 had hyperlipidemia and 11 had diabetes mellitus at the moment of the ischemic event. Twenty-eight of the 90 were current smokers and 29 had a history of smoking. 
Table 1. Participant characteristics

\begin{tabular}{|c|c|}
\hline Participant characteristics $(n=90)$ & Total number (\%) \\
\hline Mean age in years (range) & $60(27-85)$ \\
\hline Men & $52(58 \%)$ \\
\hline Mean body mass index (BMI, kg/m²), (range) & $26.1(18.1-37.5)$ \\
\hline \multicolumn{2}{|l|}{ Diagnosis } \\
\hline Ischemic stroke & $60(67 \%)$ \\
\hline Transient ischemic attack & $25(28 \%)$ \\
\hline Amaurosis fugax & $5(6 \%)$ \\
\hline Hypertension & $46(51 \%)$ \\
\hline Hyperlipidemia & 44 (49\%) \\
\hline Diabetes mellitus & $11(12 \%)$ \\
\hline Peripheral artery disease & $1(1 \%)$ \\
\hline Current smoker & $28(31 \%)$ \\
\hline Former smoker & $29(32 \%)$ \\
\hline Atrial fibrillation & $11(12 \%)$ \\
\hline Angina pectoris & $4(4 \%)$ \\
\hline Myocardial infarction & $5(6 \%)$ \\
\hline Average time between symptom onset to MRI in days ( \pm SD) & $24( \pm 34)$ \\
\hline Participants imaged without contrast agent & $7(8 \%)$ \\
\hline \multicolumn{2}{|l|}{ TOAST-criteria } \\
\hline Large-artery atherosclerosis & $47(52 \%)$ \\
\hline Cardiac embolism & $19(21 \%)$ \\
\hline Small-vessel occlusion & $5(6 \%)$ \\
\hline Other determined etiology & $8(9 \%)$ \\
\hline Undetermined & $11(12 \%)$ \\
\hline
\end{tabular}

Note-TOAST, trial of ORG 10172 in acute stroke treatment. ${ }^{22}$ 


\section{Reliability study and vessel wall lesion distribution}

The calculated agreements for the number and location of the detected vessel wall lesions were as follows: ICC of $0.93(95 \% \mathrm{Cl} ; 0.90-0.96)$ and a DSC of 0.81 . The Bland-Altman plot with 95\% levels of agreement is shown in Figure 2.

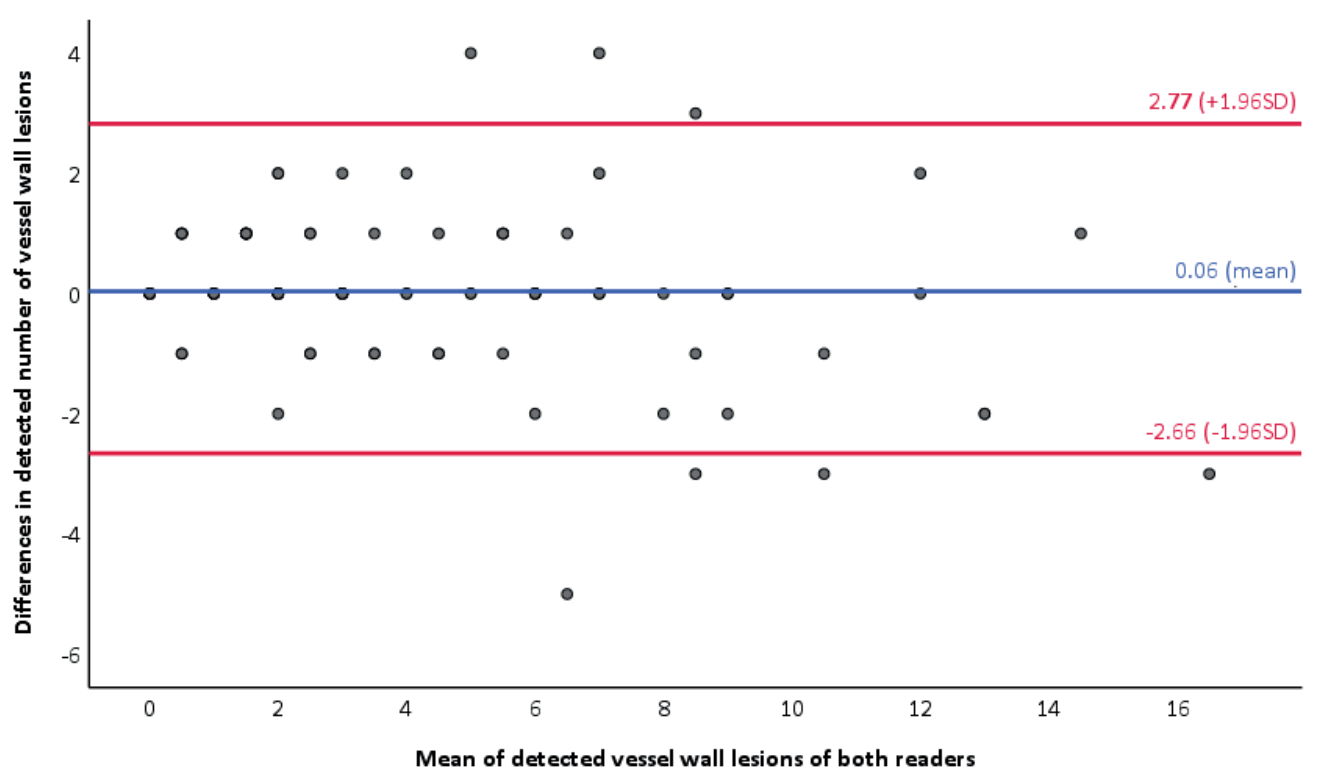

Figure 2. Bland-Altman plot shows the level of agreement in the number of detected vessel wall lesions between two readers. Differences in detected vessel wall lesions are plotted against the mean number of detected vessel wall lesions of both readers. The blue line represents the mean of differences; the two red lines reflect the $95 \%$ limits of agreement. SD, standard deviation.

Of the 90 participants included in this analysis, 60 had ischemic stroke (right MCA, $n=30$; left MCA, $n=28$; right ACA, $n=1$; left ACA, $n=1$ ); 25 had cerebral TIA (rightsided, $n=13$ ); and five had transient monocular blindness (right-sided, $n=2$ ). A total of 372 vessel wall lesions were found in 76 participants (84\%); 135 of those lesions were enhancing. In 34 of the 60 participants with an ischemic stroke, 67 vessel wall lesions (18\%) were detected in the vessels before the vascular comprised area or in the vascular territory itself, 33 of which were enhanced (49\%). An example of a participant with multiple vessel wall lesions is shown in Figure 3. More examples of intracranial vessel wall lesions are shown in Figure 4. The numbers of vessel wall lesions according to arterial segment are shown in Supplemental Table 1. 

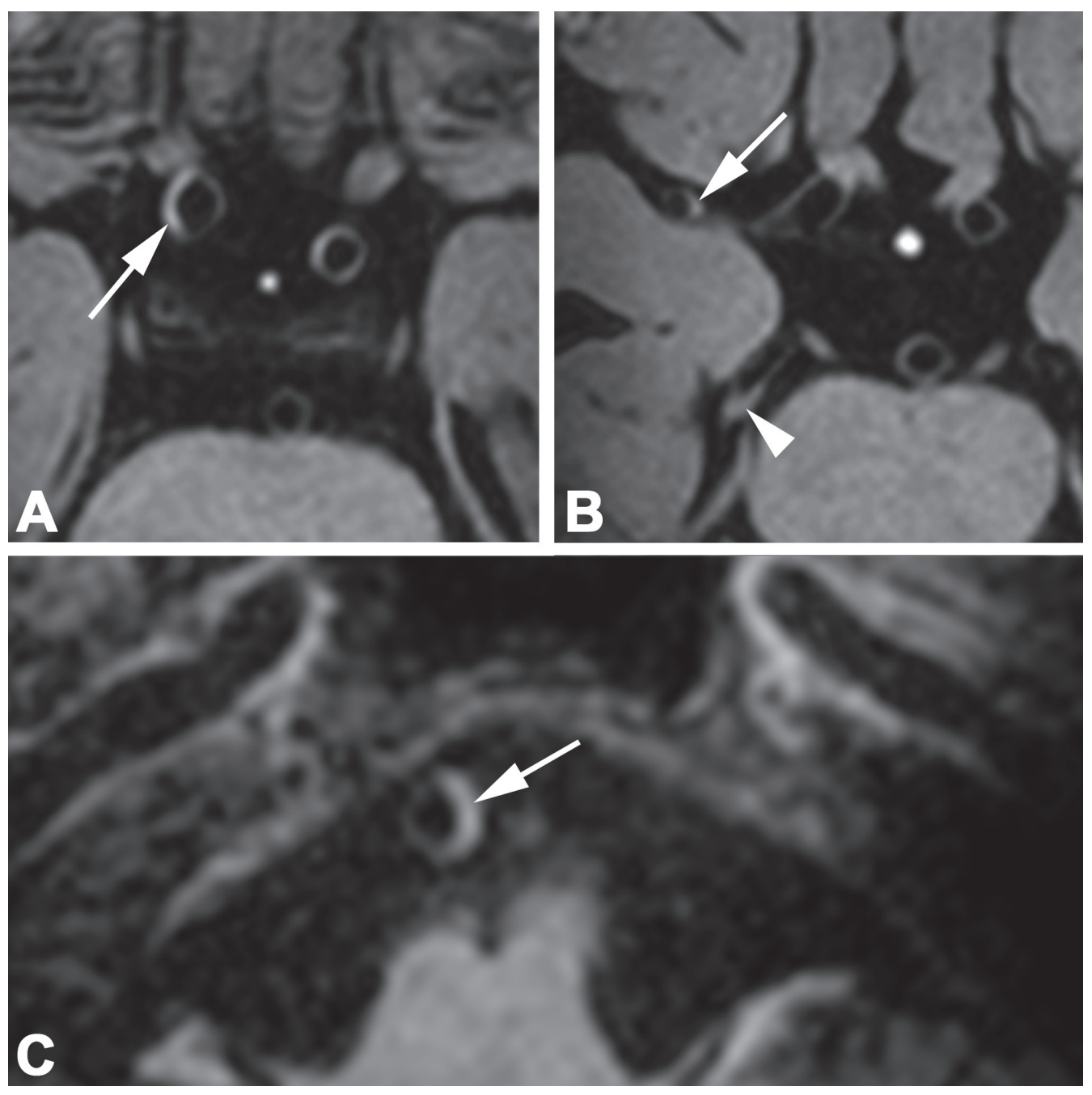

Figure 3. Images in a 69-year-old man with a left-sided ischemic stroke obtained 56 days after symptom onset. Cardiovascular risk factors present were hypertension and diabetes mellitus. The Second Manifestations of ARTerial disease (SMART) vascular risk score was 37\%, indicating a $37 \%$ chance of a recurrent vascular event within 10 years. A total of 14 vessel wall lesions were detected on the pre- and postcontrast 7T transverse 3D $\mathrm{T}_{1}$-weighted magnetization-prepared inversion-recovery TSE images (TR $3952 \mathrm{~ms}$, TE $37 \mathrm{~ms}$, TI $1375 \mathrm{~ms}$, voxel size $0.8 \times 0.8 \times 0.8 \mathrm{~mm}^{3}$ ). (A) In the anterior circulation, transverse postcontrast MRI show vessel wall thickening (white arrow) of the supra-clinoid portion of the right internal carotid artery and (B) the right M1-segment (white arrow). (B and C) In the posterior circulation, transverse postcontrast MRI show vessel wall lesions in the right P2-segment (white arrowhead in $\mathbf{B}$ ), and the basilar artery (arrow in C). 

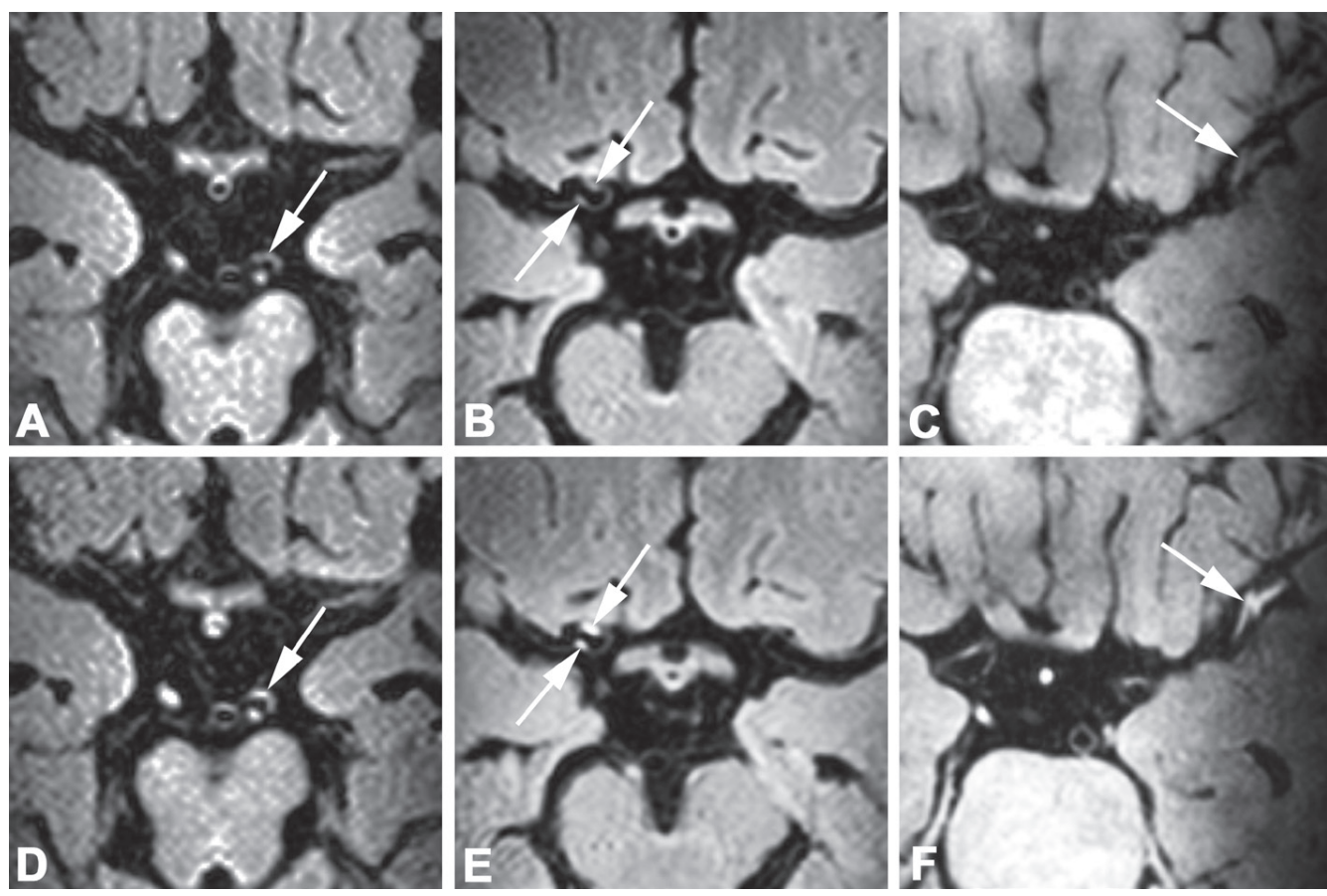

Figure 4. Images in three participants with vessel wall lesions. All participants underwent pre- and postcontrast 7T transverse 3D $\mathrm{T}_{1}$-weighted magnetization-prepared inversion-recovery TSE images (TR 3952 ms, TE 37 ms, TI 1375 ms, voxel size $0.8 \times 0.8 \times 0.8 \mathrm{~mm}^{3}$ ). (A and D) A 71-year-old man with a left-sided ischemic stroke scanned 46 days after symptom onset. Four vessel wall lesions were detected. Transverse pre- (A) and postcontrast (D) MRI show a vessel wall lesion in the left P1-segment (white arrows) that enhances after contrast agent administration. (B and E) A 46-year-old female with right-sided transient ischemic attack scanned 10 days after symptom onset. Three vessel wall lesions were detected. Transverse pre- (B) and postcontrast (E) MRI show a vessel wall lesion in the right M1-segment (white arrows) that enhances after contrast agent administration. (C and F) A 56-year-old man with a left-sided ischemic stroke scanned 65 days after symptom onset. Fifteen vessel wall lesions were detected. Transverse pre(C) and postcontrast (F) MRI show a vessel wall lesion in the left M2-segment (white arrows) that enhances after contrast agent administration. 


\section{Risk factors for vessel wall lesions}

Associations between vascular risk factors and the number of vessel wall lesions in the anterior circulation are given as unadjusted and adjusted relative risks in Table 2. After adjusting for age and sex, increasing age (adjusted only for sex) (RR, 1.02; 95\% Cl, 1.01-1.03; p-value <0.001), diabetes mellitus (RR, 1.67; $95 \% \mathrm{Cl}, 1.20-$ 2.33; p-value 0.003), and hypertension ( $R R, 1.46 ; 95 \% \mathrm{Cl}, 1.06-2.02 ; \mathrm{p}$-value 0.02) were associated with a higher number of vessel wall lesions. The multivariable vascular risk score (SMART risk score, RR, 1.01; 95\% Cl, 1.00-1.02; p-value 0.004) was also associated with a higher number of vessel wall lesions in the anterior circulation. After Bonferroni correction, increasing age remained associated with a higher number of vessel wall lesions ( $p$-value $<0.001$ ). No association was found between smoking and the number of vessel wall lesions in the anterior circulation (RR, 1.00; 95\% Cl, 0.99-1.01; p-value 0.60).

In the 83 participants who underwent both pre- and postcontrast vessel wall MRI, increasing age (RR, 1.03; 95\% Cl, 1.01-1.05; p-value 0.001) was associated with a higher number of enhancing vessel wall lesions in the anterior circulation, and this finding persisted after Bonferroni correction (Table 3). Seven participants did not undergo postcontrast imaging because of either general discomfort, or nausea and/or dizziness with imaging or technical failure (catheter dislocation).

In the 45 participants in whom both the anterior and posterior circulation could be assessed, increasing age (RR, 1.02; 95\% Cl, 1.00-1.03; p-value 0.02), hypertension (RR, 1.49; 95\% Cl, 1.01-2.21; p-value 0.046), diabetes mellitus (RR, 1.78; 95\% Cl, 1.37-2.32; p-value <0.001), higher systolic blood pressure (RR, $1.15 ; 95 \% \mathrm{Cl} 1.06$ 1.25; $p$-value 0.001), and lower eGFR (RR, 1.01; 95\% Cl 1.00-1.02; $p$-value 0.004) were associated with a higher number of vessel wall lesions, after adjusting for age and sex (Table 4). After Bonferroni correction, a higher systolic blood pressure ( $p$-value 0.001$)$ and diabetes mellitus ( $p$-value $<0.001)$ remained associated with a higher number of vessel wall lesions. Again, we did not find an association between smoking and the total number of vessel wall lesions (RR, $0.99 ; 95 \% \mathrm{Cl}$ 0.98-1.01; p-value 0.87). 


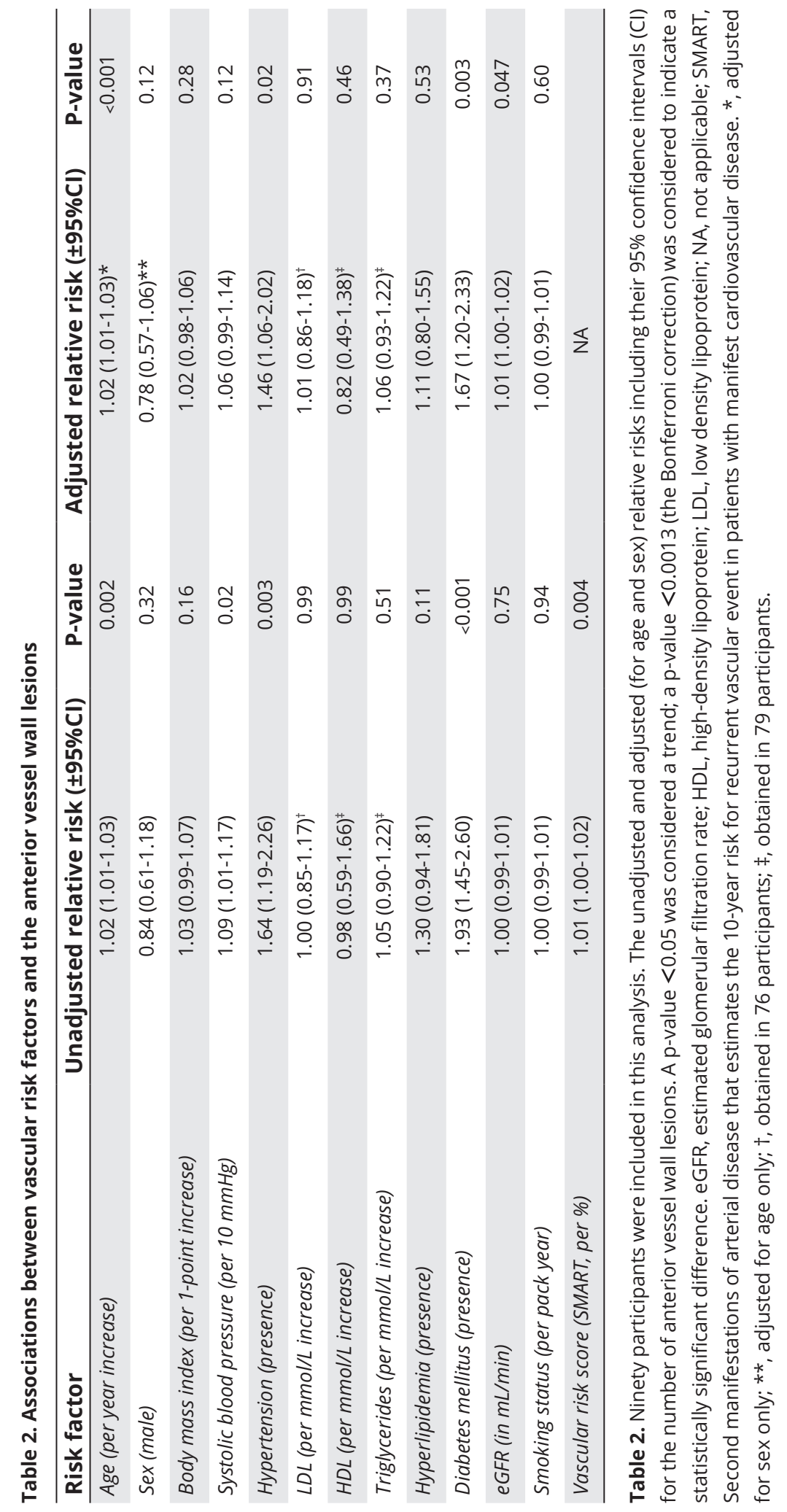




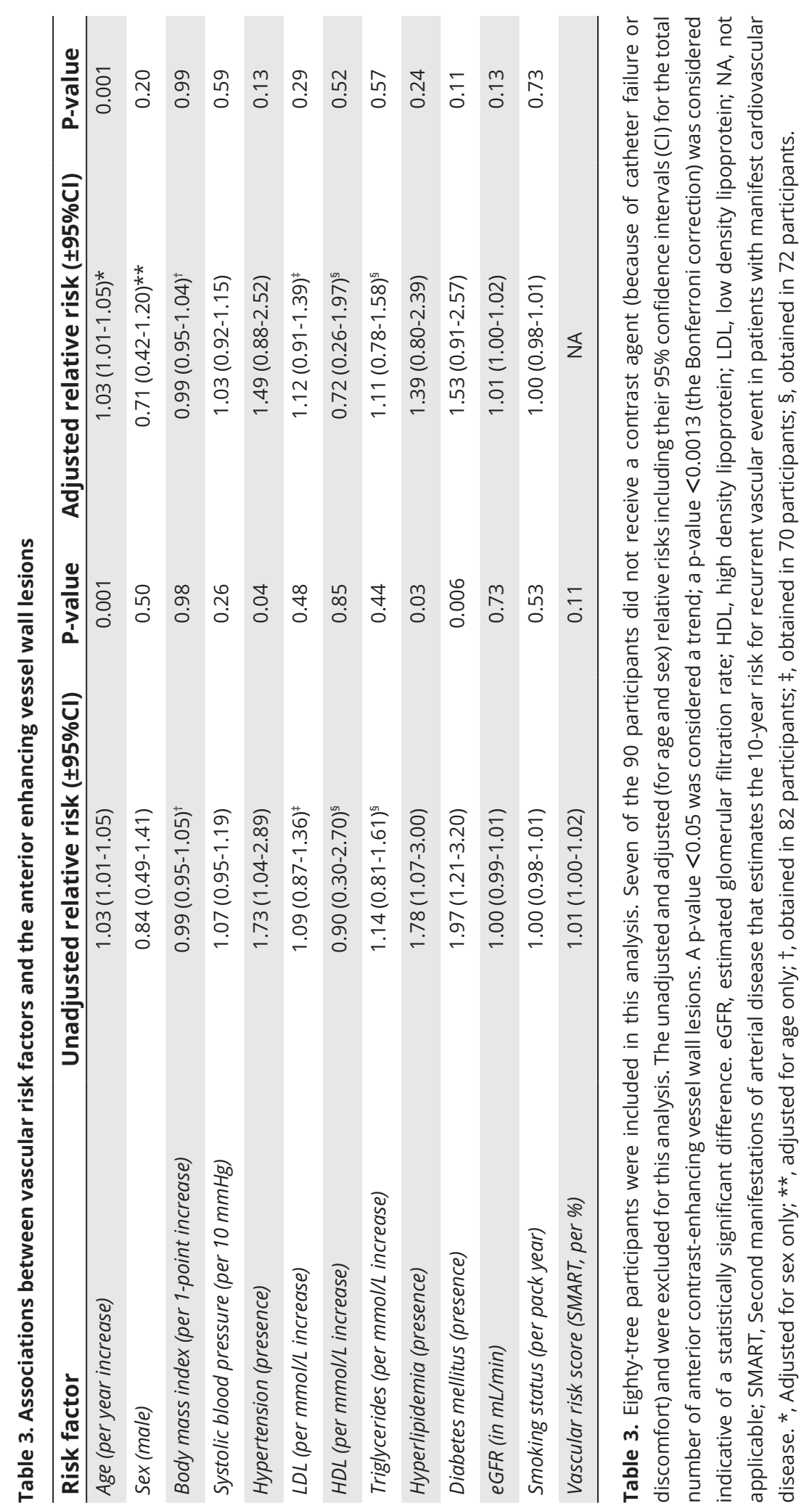




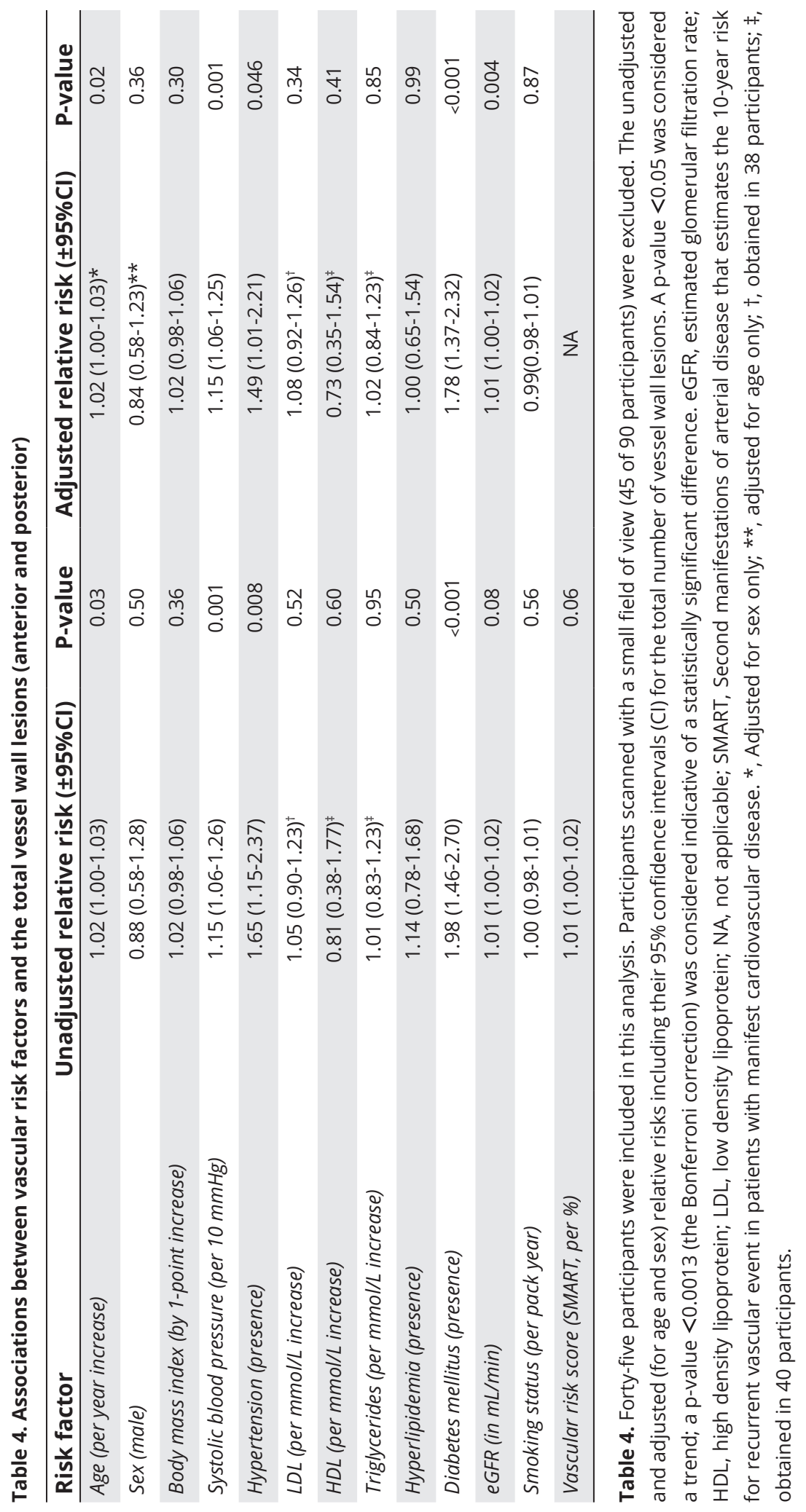




\section{Discussion}

We investigated the association between the intracranial vessel wall lesion burden assessed by using 7T MRI with dedicated intracranial vessel wall sequences and established vascular risk factors in participants with ischemic stroke or transient ischemic attack of the anterior cerebral circulation. Current data is mainly based on the assessment of luminal narrowing rather than abnormalities in the vessel wall. Our main results are threefold. First, we found that increasing age (RR, 1.02), hypertension (RR, 1.46), diabetes mellitus (RR, 1.67), and higher multivariable vascular risk score $(R R, 1.01)$ were associated with a higher vessel wall lesion burden in the anterior circulation, and that increasing age (RR, 1.03) was associated with a higher number of enhancing lesions. Second, we found that increasing age (RR, 1.02), hypertension (RR, 1.49), higher systolic blood pressure (RR, 1.15), diabetes mellitus $(R R, 1.78)$ and lower estimated glomerular filtration rate $(R R$, 1.01) were associated with vessel wall lesion burden when both anterior and posterior circulation lesions were combined. Finally, we did not find an association between smoking $(R R, 1.00)$ and the intracranial vessel wall lesion burden.

Because of heterogeneity in study populations and diagnostic methods as well as varying definitions of ICAS in previous studies, our results are difficult to compare with the literature. In these previous studies, increasing age, hypertension, and diabetes mellitus were the most consistent risk factors associated with the presence and severity of ICAS (defined as presence or degree of stenosis). ${ }^{4,8}$ This was confirmed in our study. In previous studies, the 'metabolic syndrome', defined as a constellation of risk factors, was associated with strokes caused by ICAS. ${ }^{8}$ In our study, the more elaborate composite SMART risk score was also associated with anterior circulation vessel wall lesion burden. Although smoking, dyslipidemia, and male sex have been reported as independent risk factors for ICAS in lumenographybased studies, ${ }^{4,8,33}$ no statistically significant association between these risk factors and vessel wall lesion burden was observed in our study.

A recent study that has assessed the relation between vascular risk factors and ICAS using dedicated $3 T$ intracranial vessel wall MRI sequences was performed in a community-based population in the United States that consisted of African American and white subpopulations (with oversampling of participants with cognitive impairment and previous brain MRI). ${ }^{20}$ Vascular risk factors were assessed at baseline in midlife and during follow-up, when intracranial vessel wall MRI was performed. In accordance with our study, the number of vessel wall lesions was only associated with hypertension and age in the white subpopulation. Smoking was associated with prevalence - not with lesion burden - of ICAS in the African American population and not in the white population, which is consistent with the results of our study. In contrast to our study, diabetes mellitus was associated with the prevalence of ICAS - but only 
in the African American subpopulation..$^{20}$ This difference between the studies could be caused by several factors, such as differences in study population (community-based population in the United States versus Dutch participants with an ischemic stroke or TIA). In addition, in contrast to these studies, we used the 3D MPIR-TSE sequence at 7T MRI, which has the advantage of a higher signal-to-noise and contrast-to-noise ratio, enabling optimal cerebrospinal fluid and blood suppression and clear delineation of both inner and outer vessel wall boundaries, reducing the risk of misclassification of vessel wall lesions. ${ }^{16,21,28}$

At least three hypotheses can be put forward to explain the lack of an association that we found between some of the accepted risk factors for cardiovascular disease (e.g., smoking) and intracranial vessel wall lesions. First, there may be a real difference between risk factors for extracranial and intracranial atherosclerosis, a hypothesis that is supported by previously published studies. ${ }^{8,34}$ Second, the intracranial vessel wall lesions that we scored may not only represent atherosclerotic plaques but also vessel wall thickness fluctuation due to aging, natural variation or partial volume effects, thereby underestimating any association between the risk factors and 'true' atherosclerotic lesions. So far, there has been limited validation of in vivo intracranial vessel wall MRI sequence with histologic findings. ${ }^{35}$ However, ex vivo vessel wall MRI studies have shown that lesions found with vessel wall MRI do represent intracranial atherosclerosis. ${ }^{36}$ Third, it may be that certain risk factors are only associated with ICAS in specific ethnic populations, or with more advanced stages of ICAS, in which point luminal narrowing begins to develop.

A limitation that should be considered is the relatively small number of study participants and a potential selection bias towards less-affected participants, which is inevitable in this type of MRI study. Severely disabled patients or patients with severe cardiovascular diseases treated with stent placement, pacemakers or implantable cardioverter defibrillators were not able to participate because of safety reasons. The small sample size and consequent 'selection' of lessaffected participants in theory might have led to a reversed causality with an underestimation of the true association between risk factors and vessel wall lesion burden. In addition, as we based our results on the multivariable models only, we have run three of such multivariable models (adjusted models in Table 2, 3, and 4). The application of Bonferroni correction to control for the family-wise error rate would leave increasing age, diabetes mellitus, and systolic blood pressure as the main predictors of abnormalities. Furthermore, relating vascular risk factors to the number of intracranial vessel wall lesions is challenging. Vascular risk factors are dependent on several aspects, such as severity of exposure status, lifestyle behavior, medication adherence, and genetic susceptibility. Therefore, it is not possible to include all potential confounders, effect modifiers and residual biases into one model. Studies with a larger number of patients that include 
healthy controls and meta-analyses elaborating a detailed risk factor analysis may contribute to interpretation of the exact association of these cardiovascular risk factors with the intracranial vessel wall lesion burden.

In conclusion, increasing age, hypertension, diabetes mellitus, and a higher composite Second Manifestations of ARTerial disease (SMART) risk score are associated with a higher number of intracranial vessel wall lesions when assessed with 7TMRI in white patients with stroke or transient ischemic attack. We could not find an association between smoking and the intracranial vessel wall lesion burden. 


\section{References}

1. Arenillas JF. Intracranial atherosclerosis: current concepts. Stroke 2011;42:S20-3.

2. Holmstedt CA, Turan TN, Chimowitz MI. Atherosclerotic intracranial arterial stenosis: risk factors, diagnosis, and treatment. The Lancet Neurology 2013;12:1106-14.

3. Qureshi Al, Caplan LR. Intracranial atherosclerosis. Lancet 2014;383:984-98.

4. Gorelick P, Wong KS, Liu L. Epidemiology. Frontiers of Neurology and Neuroscience 2016;40:34-46.

5. Bos $\mathrm{D}$, Portegies $\mathrm{ML}$, van der Lugt $\mathrm{A}$, et al. Intracranial carotid artery atherosclerosis and the risk of stroke in whites: the Rotterdam Study. JAMA Neurology 2014;71:405-11.

6. Homburg PJ, Plas GJ, Rozie S, van der Lugt A, Dippel DW. Prevalence and calcification of intracranial arterial stenotic lesions as assessed with multidetector computed tomography angiography. Stroke 2011;42:1244-50.

7. Arenillas JF, Lopez-Cancio E, Wong KS. Biomarkers, Natural Course and Prognosis. Frontiers of Neurology and Neuroscience 2016;40:93-108.

8. Uehara T, Bang OY, Kim JS, Minematsu K, Sacco R. Risk Factors. Frontiers of Neurology and Neuroscience 2016;40:47-57.

9. Rincon $F$, Sacco RL, Kranwinkel $G$, et al. Incidence and risk factors of intracranial atherosclerotic stroke: the Northern Manhattan Stroke Study. Cerebrovasc Dis 2009;28:65-71.

10. Chaturvedi S, Turan TN, Lynn MJ, et al. Risk factor status and vascular events in patients with symptomatic intracranial stenosis. Neurology 2007;69:2063-8.

11. Nguyen-Huynh MN, Wintermark M, English J, et al. How accurate is CT angiography in evaluating intracranial atherosclerotic disease? Stroke 2008;39:1184-8.

12. Navarro JC, Lao AY, Sharma VK, Tsivgoulis G, Alexandrov AV. The accuracy of transcranial Doppler in the diagnosis of middle cerebral artery stenosis. Cerebrovasc Dis 2007;23:325-30.

13. Qiao Y, Anwar Z, Intrapiromkul J, et al. Patterns and Implications of Intracranial Arterial Remodeling in Stroke Patients. Stroke 2016;47:434-40.

14. Gutierrez J, Goldman J, Honig LS, Elkind MS, Morgello S, Marshall RS. Determinants of cerebrovascular remodeling: do large brain arteries accommodate stenosis? Atherosclerosis 2014;235:371-9.

15. Dieleman N, Kolk AG, Zwanenburg JJ, Harteveld AA, Biessels GJ, Luijten PR. Imaging intracranial vessel wall pathology with magnetic resonance imaging: current prospects and future directions. Circulation 2014;130:192-201.

16. Van der Kolk AG, Zwanenburg JJ, Brundel M, et al. Intracranial vessel wall imaging at 7.0T MRI. Stroke 2011;42:2478-84.

17. Qiao Y, Steinman DA, Qin Q, Etesami M, Schar M, Astor BC. Intracranial arterial wall imaging using three-dimensional high isotropic resolution black blood MRI at 3.0 Tesla. Journal of magnetic resonance imaging: JMRI 2011;34:22-30. 
18. Lindenholz A, van der Kolk AG, Zwanenburg JJM, Hendrikse J. The Use and Pitfalls of Intracranial Vessel Wall Imaging: How We Do It. Radiology 2018;286:12-28.

19. Mandell DM, Mossa-Basha M, Qiao Y, et al. Intracranial Vessel Wall MRI: Principles and Expert Consensus Recommendations of the American Society of Neuroradiology. AJNR American Journal of Neuroradiology 2017;38:218-229.

20. Qiao Y, Suri FK, Zhang Y, et al. Racial Differences in Prevalence and Risk for Intracranial Atherosclerosis in a US Community-Based Population. JAMA Cardiology 2017;12:13411348.

21. Van der Kolk AG, Hendrikse J, Brundel M, Biessels GJ, Smit EJ, Visser F. Multi-sequence whole-brain intracranial vessel wall imaging at 7.0 tesla. European Radiology 2013;23:2996-3004.

22. Adams HP, Jr., Bendixen BH, Kappelle LJ, et al. Classification of subtype of acute ischemic stroke. Definitions for use in a multicenter clinical trial. TOAST. Trial of Org 10172 in Acute Stroke Treatment. Stroke 1993;24:35-41.

23. Piepoli MF, Hoes AW, Agewall S, et al. 2016 European Guidelines on cardiovascular disease prevention in clinical practice. European Heart Journal 2016;37:2315-81.

24. Ryden L, Grant PJ, Anker SD, et al. ESC Guidelines on diabetes, pre-diabetes, and cardiovascular diseases. European Heart Journal 2013;34:3035-87.

25. Catapano AL, Graham I, De Backer G, et al. 2016 ESC/EAS Guidelines for the Management of Dyslipidaemias. European Heart Journal 2016;37:2999-3058.

26. Dorresteijn JA, Visseren FL, Wassink AM, et al. Development and validation of a prediction rule for recurrent vascular events based on a cohort study of patients with arterial disease: the SMART risk score. Heart 2013;99:866-72.

27. Dieleman N, van der Kolk AG, Zwanenburg J, et al. Relations between location and type of intracranial atherosclerosis and parenchymal damage. Journal of cerebral blood flow and metabolism: 2016;36:1271-80.

28. Harteveld AA, van der Kolk AG, van der Worp HB, et al. High-resolution intracranial vessel wall MRI in an elderly asymptomatic population: comparison of 3T and 7T. European Radiology 2017;27:1585-1595.

29. Klein S, Staring M, Murphy K, Viergever MA, Pluim JP. elastix: a toolbox for intensity-based medical image registration. IEEE Transactions on Medical Imaging 2010;29:196-205.

30. Portanova A, Hakakian N, Mikulis DJ, Virmani R, Abdalla WM, Wasserman BA. Intracranial vasa vasorum: insights and implications for imaging. Radiology 2013;267:667-79.

31. Kuijf HJ, van Veluw SJ, Viergever MA, Vincken KL, Biessels GJ. How to assess the reliability of cerebral microbleed rating? Frontiers in Aging Neuroscience 2013;5:57.

32. Altman DG, Royston P. The cost of dichotomising continuous variables. BMJ 2006;332:1080.

33. Sacco RL, Kargman DE, Gu Q, Zamanillo MC. Race-ethnicity and determinants of intracranial atherosclerotic cerebral infarction. The Northern Manhattan Stroke Study. Stroke 1995;26:14-20. 
34. Kim JS, Nah HW, Park SM, et al. Risk factors and stroke mechanisms in atherosclerotic stroke: intracranial compared with extracranial and anterior compared with posterior circulation disease. Stroke 2012;43:3313-8.

35. Yang WJ, Wong KS, Chen XY. Intracranial Atherosclerosis: From Microscopy to HighResolution Magnetic Resonance Imaging. Journal of Stroke 2017;19:249-60.

36. Harteveld AA, Denswil NP, Siero JC, et al. Quantitative Intracranial Atherosclerotic Plaque Characterization at 7T MRI: An Ex Vivo Study with Histologic Validation. AJNR American Journal of Neuroradiology 2016;37:802-10. 


\section{Supplemental Material}

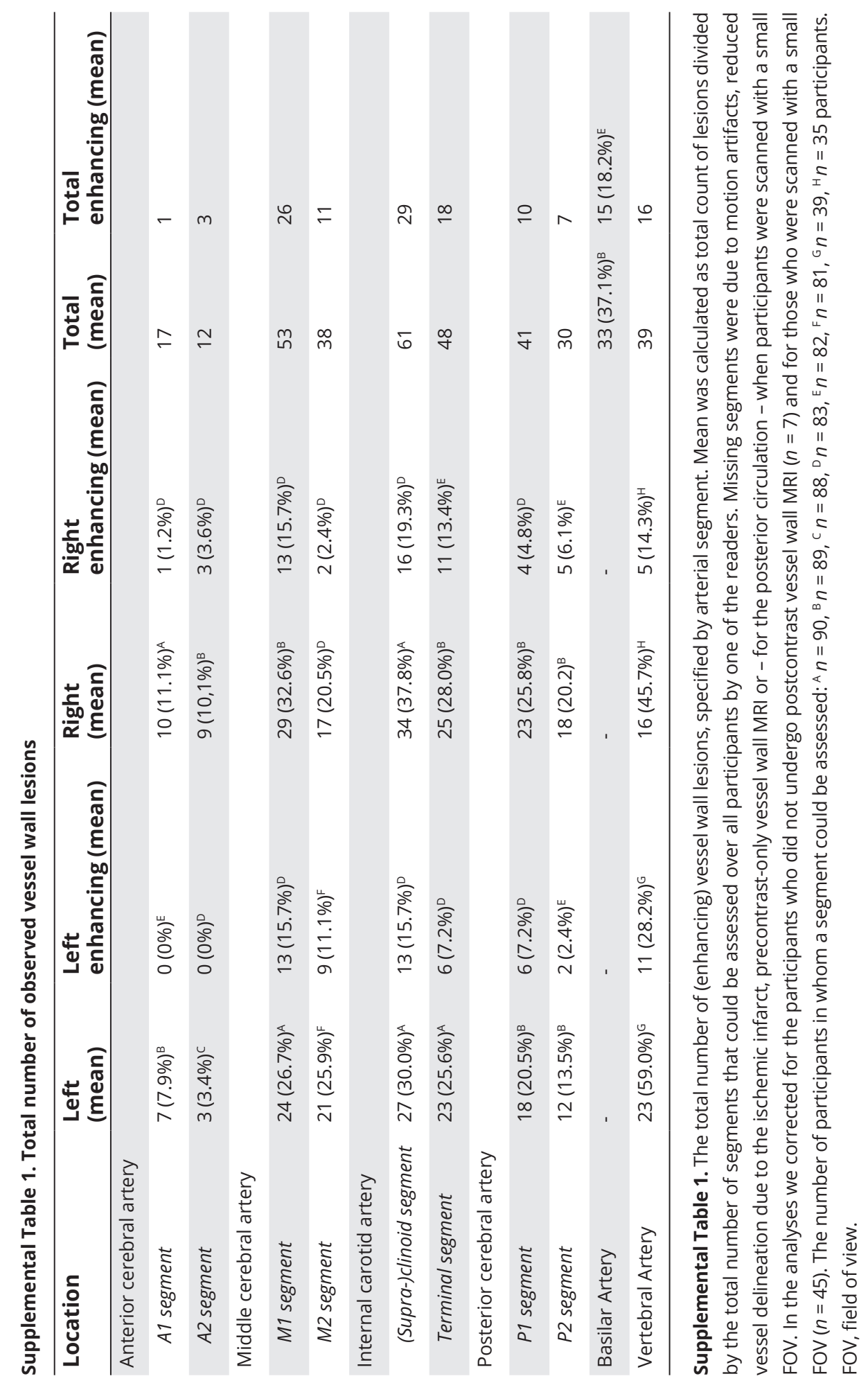




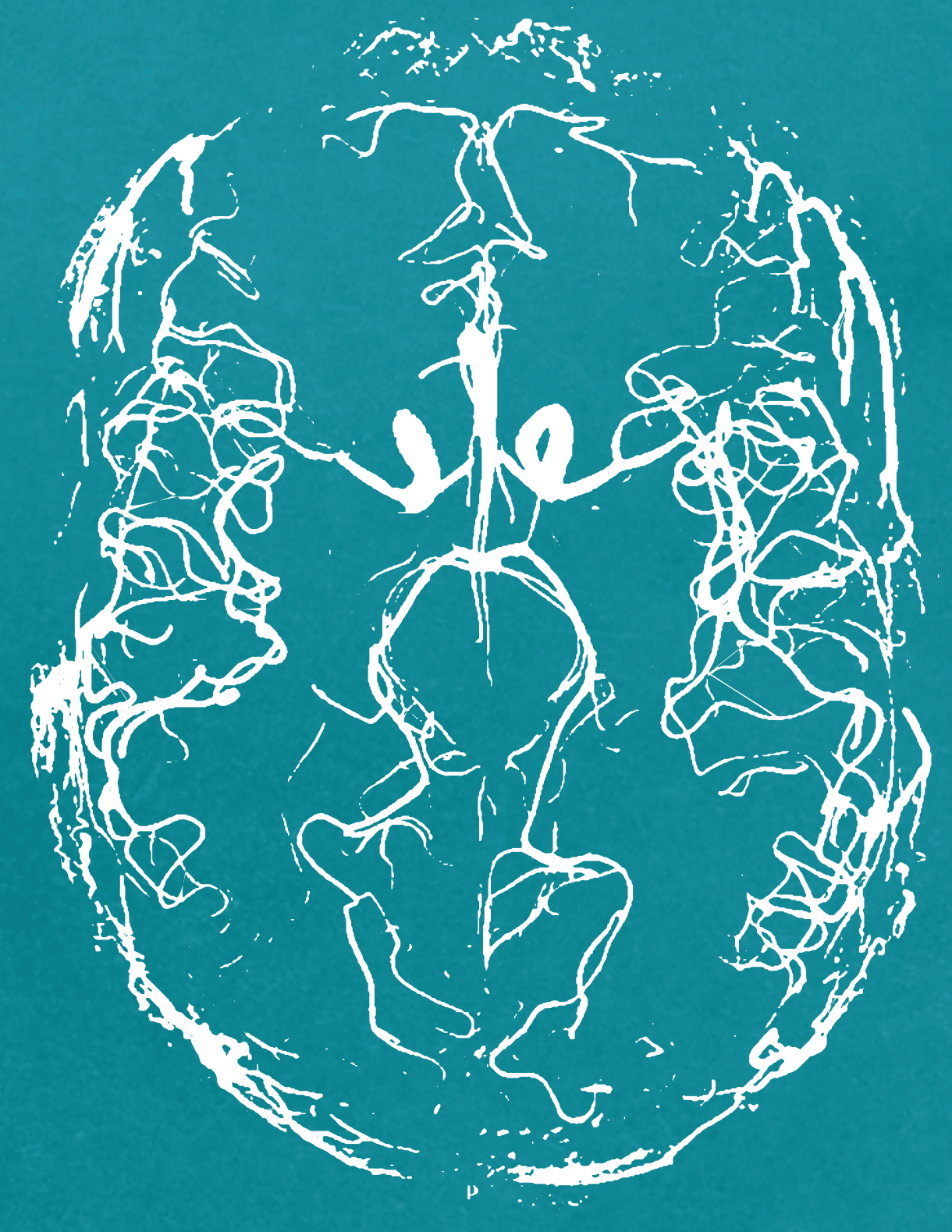




\section{CHAPTER}

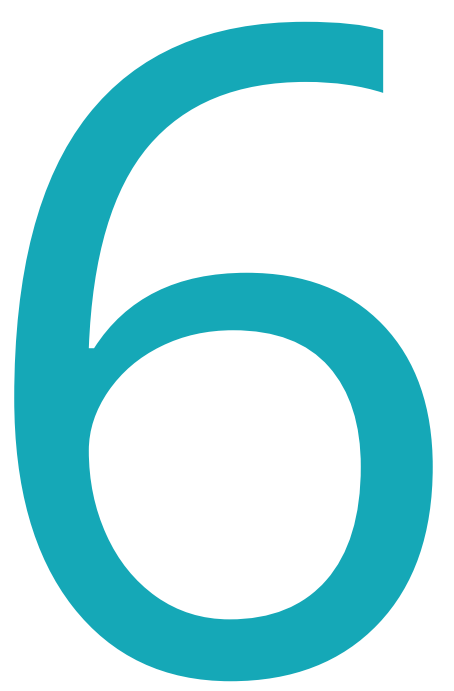

\section{Intracranial Atherosclerotic Burden and Cerebral Parenchymal Changes at 7T MRI in Patients with TIA or Ischemic Stroke}

Arjen Lindenholz', Jeroen de Bresser ${ }^{2}$, Anja G. van der Kolk¹, H. Bart van der Worp ${ }^{3}$, Theodoor D. Witkamp ${ }^{1}$, Jeroen Hendrikse ${ }^{1}$ and Irene C. van der Schaaf ${ }^{1}$

1 Department of Radiology, University Medical Center Utrecht, The Netherlands ${ }^{2}$ Department of Radiology, Leiden University Medical Center, The Netherlands ${ }^{3}$ Department of Neurology and Neurosurgery, University Medical Center Utrecht, Brain Center Rudolf Magnus, Utrecht University, The Netherlands

Published in Frontiers of Neurology, section Stroke (Research Topic: Cerebrovascular Imaging - from micro- tot macroscopic scales), 2021. 


\begin{abstract}
The relevance of intracranial vessel wall lesions detected with MRI is not fully established. In this study (trial identification number: NTR2119; www.trialregister.nl) 7T MRI was used to investigate if a higher vessel wall lesion burden is associated with more cerebral parenchymal changes in patients with ischemic stroke or TIA. MR images of 82 patients were assessed for the number of vessel wall lesions of the large intracranial arteries and for cerebral parenchymal changes, including the presence and number of cortical, small subcortical and deep grey matter infarcts, lacunes of presumed vascular origin, cortical microinfarcts, and periventricular and deep white matter hyperintensities (WMH). Regression analyses showed that a higher vessel wall lesion burden was associated with the presence of small subcortical infarcts, lacunes of presumed vascular origin, deep grey matter infarcts (relative risk 1.18; $95 \% \mathrm{Cl} 1.03-1.35$ ), and presence of moderate to severe periventricular $\mathrm{WMH}(1.21 ; 95 \% \mathrm{Cl} 1.03-1.42)$ which are all manifestations of small vessel disease (SVD). The burden of enhancing vessel wall lesions was associated with the number of cortical microinfarcts only $(1.48 ; 95 \% \mathrm{Cl} 1.04-2.11)$. These results suggest an interrelationship between large vessel wall lesion burden and cerebral parenchymal manifestations often linked to SVD or, alternatively, that vascular changes occur in both large and small intracranial arteries simultaneously.
\end{abstract}




\section{Introduction}

Intracranial atherosclerosis (ICAS) is an important cause of ischemic stroke. ${ }^{1}$ Historically, ICAS has been evaluated by measuring the presence of intracranial stenosis using lumenographic techniques, or by detecting vessel wall calcifications that generally reflect a more advanced stage of ICAS..$^{2-5}$ Over the last two decades, however, intracranial vessel wall MRI sequences have enabled in vivo visualization of the intracranial vessel wall itself. ${ }^{6-8}$ With these dedicated MRI sequences, both subtle (non-stenotic) and more advanced vessel wall pathology of the proximal cerebral large arteries can be assessed.

Vessel wall changes of the large intracranial arteries are frequently observed on intracranial vessel wall MR images, both in patients with cerebrovascular disease as well as in healthy elderly individuals, but the nature and clinical relevance of these changes have not been fully established. ${ }^{9-12}$ Ex vivo studies applying intracranial vessel wall MRI to postmortem samples suggest that these changes represent vessel wall disease at an early stage to halfway on the developmental timeline of ICAS. ${ }^{13-18}$ At the end of this timeline, plaque disruption, thrombus formation and large vessel stenosis or occlusion can lead to transient ischemic attack (TIA) or ischemic stroke. The parenchymal consequences of vessel wall changes presumably reflecting less advanced ICAS, however, are less clear, hampering interpretation of these changes in clinical practice.

ICAS in the smaller arteries, arterioles and capillaries - while difficult to visualize using vessel wall MRI due to their small caliber - or emboli disrupted from atherosclerotic plaques located in larger parent arteries may lead to (small) subcortical infarcts, deep grey matter infarcts, lacunes of presumed vascular origin, cortical microinfarcts or white matter hyperintensities. ${ }^{19,20}$ Often, these cerebrovascular changes of the small vessels and their sequelae are categorized as a separate disease entity (small vessel disease, SVD) compared with large artery stroke, although both small and large cerebral arteries comprise one vascular bed that is physiologically connected, suggesting that pathology in one 'type' of vessel bed will inevitably affect the other. Recent studies have shown evidence for this connection: for example, large (intracranial) artery disease may result in endothelial damage and blood-brain barrier damage of the smaller arteries by the release of inflammatory molecules and enzymes, while alternatively, atherosclerotic plaques located in the larger intracranial arteries may obstruct orifices of smaller branching arteries resulting in small vessel pathology. ${ }^{21,22}$ Nevertheless, the exact role and direction of effect - cerebrovascular changes of the small arteries by large artery pathology, vice versa, or bilateral - remains to be elucidated. ${ }^{22}$ 
In this study, we hypothesize that the burden of intracranial vessel wall lesions of the larger intracranial arteries might be associated with a variety of cerebral parenchymal changes, including large and small infarcts and white matter hyperintensities. The purpose of this study was therefore to investigate whether a greater burden of intracranial vessel wall lesions assessed using 7 tesla $(T)$ vessel wall MRI is associated with more cerebral parenchymal changes.

\section{Materials and Methods}

\section{Study population}

Between December 2009 and May 2018, patients presenting at our university hospital with TIA or ischemic stroke of the anterior circulation were eligible for inclusion in the prospective Intracranial Vessel wall Imaging (IVI) study (NTR2119, www.trialregister.nl). The main inclusion criteria were age 18 years or older and ability to undergo a 7T MRI examination within three months after the ischemic event. The main exclusion criteria were ischemic stroke or TIA secondary to a surgical or interventional procedure and any contraindication to 7T MR imaging or to gadolinium-containing contrast agents. A flowchart of the study inclusion is shown in Figure 1. The full eligibility criteria and other details of the study have been reported before. ${ }^{6,23}$ The study was approved by the medical ethics committee of the University Medical Center Utrecht according to the guidelines of the Declaration of Helsinki of 1975 and all patients provided written informed consent. Baseline characteristics including cardiovascular risk factors and characteristics of the ischemic event (final diagnosis and subtype classified by the Trial of Org 10172 in Acute Stroke Treatment (TOAST) criteria) were recorded. ${ }^{24}$

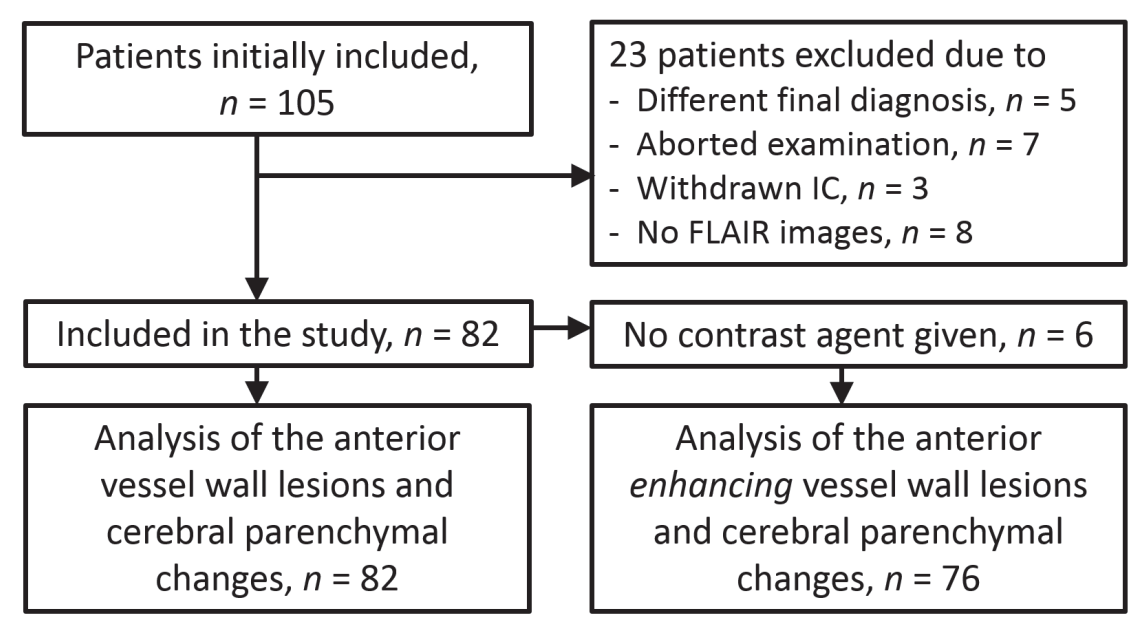

Figure 1: Flowchart showing the number of participants who were included for analyses. IC = informed consent. 


\section{Imaging protocol}

Imaging was performed on a 7T whole-body system (Philips Healthcare, Best, The Netherlands) with either a 16-channel $(n=35)$ or a 32-channel $(n=47)$ receive coil and a volume transmit-receive coil for transmission (Quad TR; Nova Medical, Wilmington, MA, USA). The protocol included a pre- and postcontrast $\mathrm{T}_{1}$-weighted intracranial vessel wall sequence (magnetization-prepared inversion recovery turbo spin-echo, MPIR-TSE), time-of-flight MR angiography (TOF-MRA), diffusion-weighted imaging (DWI) and fluid-attenuated inversion recovery (FLAIR) imaging. The postcontrast MPIR-TSE sequence was acquired five minutes after the administration of $0.1 \mathrm{~mL} / \mathrm{kg}$ of a gadolinium-containing contrast agent (gadobutrol, Gadovist $1.0 \mathrm{mmol} / \mathrm{mL}$, Bayer Schering Pharma, Newbury, UK). The TOF-MRA images were used for anatomical verification of the large intracranial vessels seen on the MPIR-TSE images. The following acquisition parameters were used for the MPIR-TSE sequence: field-of-view (FOV) $220 \times 180 \times 13 \mathrm{~mm}^{3}(n=40)$, which was extended during the study period to $250 \times 250 \times 190 \mathrm{~mm}^{3}(n=42){ }^{23}$ acquired spatial resolution $0.8 \times 0.8 \times 0.8 \mathrm{~mm}^{3}$, reconstructed spatial resolution $0.5 \times 0.5 \times 0.5 \mathrm{~mm}^{3}$, repetition time (TR) $3952 \mathrm{~ms}$, echo time (TE) $37 \mathrm{~ms}$, inversion time (TI) $1375 \mathrm{~ms}$, and acquisition time 10 minutes 40 seconds. In case of the smaller FOV sequence, the FOV was placed in such a way that all large intracranial arteries of the anterior circulation were within the imaging plane, which included the anterior cerebral arteries (ACA; $A 1$ and $A 2$ segments), middle cerebral arteries (MCA; M1 and M2 segments), and the distal parts of the internal (intracranial) carotid arteries (ICA; clinoid, supra-clinoid and terminal segments). Acquisition parameters for the other sequences were as follows: for the TOF-MRA, FOV 190 $\times 190 \times 102 \mathrm{~mm}^{3}$, acquired spatial resolution $0.4 \times 0.5 \times 0.6 \mathrm{~mm}^{3}$, reconstructed spatial resolution $0.4 \times 0.4 \times 0.3 \mathrm{~mm}^{3}$, TR $21 \mathrm{~ms}$, TE $2.3 \mathrm{~ms}$, and acquisition time 9 minutes 18 seconds; for the DWI, FOV $220 \times 220 \times 123 \mathrm{~mm}^{3}$, acquired and reconstructed spatial resolution $1.5 \times 1.5 \times 1.5 \mathrm{~mm}^{3}$, TR $17659 \mathrm{~ms}$, TE $57 \mathrm{~ms}$, and acquisition time 6 minutes 10 seconds; and for the FLAIR sequence, FOV $250 \times 250$ $\times 190 \mathrm{~mm}^{3}$, acquired spatial resolution $0.8 \times 0.8 \times 0.4 \mathrm{~mm}^{3}$, reconstructed spatial resolution $0.5 \times 0.5 \times 0.5 \mathrm{~mm}^{3}$, TR $8000 \mathrm{~ms}$, TE $300 \mathrm{~ms}$, TI $2200 \mathrm{~ms}$, and acquisition time 10 minutes 48 seconds (for more details see reference ${ }^{11}$ ).

\section{Vessel wall assessment}

The MR vessel wall images were scored by an expert in reading intracranial vessel wall images (AK, 9 years of experience). The reader was blinded for patient details and clinical information. Interrater reproducibility measures for the assessment of these vessel wall images have been published before (intraclass correlation coefficient, 0.93; 95\% Cl; 0.90-0.96; Dice similarity coefficient (DSC), 0.81.25 For the current study, intracranial vessels of the anterior circulation were assessed, including the ACA ( $\mathrm{A} 1$ and $\mathrm{A} 2$ segments), MCA (M1 and M2 segments) and the ICA 
(clinoid, supra-clinoid and terminal segments). A vessel wall lesion was defined as a visually judged focal or diffuse vessel wall thickening of $>50 \%$ compared with the neighboring vessel wall (or contralateral vessel wall in case of diffuse thickening), with or without contrast enhancement. ${ }^{11}$ Contrast enhancement was assessed by comparing pre- and postcontrast images including co-registered subtraction images using the Elastix toolbox in MeVisLab (version 2.7, MeVis Medical Solutions, Bremen, Germany). ${ }^{26}$ Contrast enhancement of a vessel wall lesion was defined as lesion hyperintensity approximating the signal intensity of the pituitary stalk on the postcontrast MR images on at least two consecutive images using the precontrast MR images as a reference and the coregistered subtraction images as confirmation. Contrast enhancing foci at the location where the ICA pierces the dura mater were classified as vasa vasorum and were not considered vessel wall enhancement. The intracranial vessel wall lesion burden was used as a continuous variable for the primary analysis, and was defined as the total number of vessel wall lesions for each patient. The total number of enhancing vessel wall lesions was used as a continuous variable in the secondary analysis.

\section{Parenchyma assessment}

The DWI and FLAIR images and $T_{1}$-weighted MPIR-TSE images were assessed for anterior circulation parenchymal changes by an expert neuroradiologist (TDW, 30 years of experience). The reader was blinded for patient details, clinical information and vessel wall lesion assessment. The following cerebral parenchymal changes were scored: cortical infarcts and (recent) small subcortical infarcts, deep grey matter infarcts and lacunes of presumed vascular origin, cortical microinfarcts and periventricular and deep white matter hyperintensities (WMH). A recent position paper has judged all of these MR findings except for cortical infarcts (and possibly cortical microinfarcts) to be related to SVD. ${ }^{27}$

Cortical infarcts were defined as hyperintense lesions on FLAIR imaging located in the cerebral cortex and greater than $5 \mathrm{~mm}$ in diameter, with or without associated tissue loss or extension into the deep white matter. When a cortical infarct was multifocal or extending into the white matter, but with similar presumed origin, the infarct was scored as a single cortical infarct. Small subcortical infarcts and lacunes of presumed vascular origin were defined as hyperintense lesions of respectively $15-20 \mathrm{~mm}$ and $3-15 \mathrm{~mm}$ in diameter on FLAIR imaging with or without a hypointense center or cavity and local tissue loss, classified and scored according to the STRIVE criteria. ${ }^{27}$ Deep grey matter infarcts were defined as hyperintense lesions on FLAIR imaging located in the basal ganglia or thalamus greater than $3 \mathrm{~mm}$ in diameter (not specifically defined in the STRIVE criteria but derived from the position paper. ${ }^{27}$ Cortical microinfarcts were defined as hyperintense lesions on FLAIR imaging in the cerebral cortex smaller than $5 \mathrm{~mm}$ 
in size and scored according to a recent consensus paper. ${ }^{20}$ The periventricular and deep white matter hyperintensities on FLAIR imaging were also defined according to the STRIVE criteria and were scored with the Fazekas four-point scale for periventricular (0: absent WMH lesions, 1: 'caps' or pencil-thin lining, 2: smooth 'halo' and 3: irregular hyperintensities extending into the deep white matter) and for deep WMH (0: absence or a single punctate WMH lesion, 1: multiple punctate lesions, 2: beginning confluency of lesions and 3: large confluent lesions). ${ }^{27,28}$ The presence of any cerebral infarct in the flow territory of the anterior circulation and the WMH score (dichotomized into high; Fazekas 2 or 3 and low; Fazekas 0 or 1) were used as primary outcomes in the analyses. The number of cerebral infarcts were used as outcomes for the secondary analysis.

\section{Statistical Analysis}

Descriptive baseline statistics are presented as proportions or means. To estimate associations between intracranial vessel wall lesion burden and presence or number of cerebral parenchymal changes, appropriate regression analyses for modeling count data were performed. Age and sex were included as covariates. In the primary analyses, the association between the number of intracranial vessel wall lesions (as continuous and independent variable) and the presence of any scored cerebral parenchymal change (dichotomized outcome as dependent variable) were individually and all together investigated with a negative logbinomial regression model. A composite variable was composed with the infarcts that are often considered to be manifestations of cerebral small vessel disease, and included small subcortical and deep grey matter infarcts and lacunes of presumed vascular origin. ${ }^{27}$ In the secondary analyses, the associations between the total number of intracranial vessel wall lesions (independent variable) and the total number of infarcts (count data; classified by infarct type, dependent variable) were investigated with a negative log-binomial regression model for the number of infarcts. To assess the association between the number of enhancing intracranial vessel wall lesions (independent variable) and presence and number of the scored cerebral parenchymal changes as dependent variables we used the same methods as in the primary and secondary analysis. Negative log-binomial regression generally provides rate ratios, which are interpretable as relative risks. Therefore, associations are presented in more commonly used relative risks. For all analyses 95\% confidence intervals ( $\mathrm{Cl}$ ) are given. A two-sided $\mathrm{p}$-value $<0.05$ was considered statistically significant. Statistical analyses were performed using SPSS version 21.0 (IBM SPSS Statistics, IBM Corp., Armonk, NY, USA). 


\section{Results}

A total of 105 patients were included in the IVI study. Twenty-three patients were excluded because of reasons listed in Figure 1, leaving a total of 82 patients for the current study. Baseline characteristics of the study participants are shown in Table 1.

Table 1. Baseline characteristics.

\begin{tabular}{|c|c|}
\hline Patient characteristics $(n=82)$ & Total number (\%) \\
\hline Mean age in years (range) & $61(27-85)$ \\
\hline Men & $49(60 \%)$ \\
\hline Body mass index (BMI, kg/m²), mean (range) & $26(18-35)$ \\
\hline \multicolumn{2}{|l|}{ Diagnosis } \\
\hline Ischemic stroke & 55 (67\%) \\
\hline Transient ischemic attack & $22(27 \%)$ \\
\hline Transient monocular visual loss of vascular origin & $5(6 \%)$ \\
\hline Hypertension & $40(49 \%)$ \\
\hline Hyperlipidemia & $39(48 \%)$ \\
\hline Diabetes mellitus & $10(12 \%)$ \\
\hline Peripheral artery disease & $0(0 \%)$ \\
\hline Current smoker & $26(32 \%)$ \\
\hline Former smoker & $27(33 \%)$ \\
\hline Atrial fibrillation & $10(12 \%)$ \\
\hline Angina pectoris & $4(5 \%)$ \\
\hline Myocardial infarction & $5(6 \%)$ \\
\hline Average days from symptom onset to MRI in days \pm SD & $23 \pm 34$ \\
\hline Patients imaged without contrast agent & $6(7 \%)$ \\
\hline \multicolumn{2}{|l|}{ TOAST-criteria } \\
\hline Large artery atherosclerosis & $44(54 \%)$ \\
\hline Cardio embolism & $17(21 \%)$ \\
\hline Small-vessel occlusion & $5(6 \%)$ \\
\hline Other determined etiology & $5(6 \%)$ \\
\hline Undetermined & $11(13 \%)$ \\
\hline
\end{tabular}

Table 1. Patient characteristics for the studied population. 


\section{Distribution of intracranial vessel wall lesions and cerebral parenchymal changes}

Sixty-two patients (75\%) had a total of 193 vessel wall lesions and 35 patients (43\%) a total of 81 enhancing vessel wall lesions. The total number of (enhancing) vessel wall lesions per arterial segment is shown in Supplemental Table 1.

Sixty-eight patients (83\%) had at least one cerebral parenchymal vascular lesion. Fifty-five patients (67\%) had a total of 122 cortical infarcts. One patient $(1 \%)$ had a small subcortical infarct of $<20 \mathrm{~mm}, 23$ patients $(28 \%)$ had a total of 54 lacunes of presumed vascular origin, 15 patients (18\%) had a total of 21 subcortical deep grey matter infarcts, and 16 patients (20\%) had a total of 43 cortical microinfarcts. For the periventricular WMH 24 patients (29\%) and for the deep WMH 25 patients (30\%) had a Fazekas score of 2 or 3 . All details regarding the detected cerebral parenchymal changes are shown in Supplemental Table 2.

\section{Intracranial vessel wall lesion burden and cerebral parenchymal changes}

Unadjusted and adjusted relative risks for the association between intracranial vessel wall lesion burden and the presence of cerebral parenchymal changes are shown as primary analyses in Table 2 . In our study population, vessel wall lesion burden was not associated with the presence of small cortical infarcts (adjusted relative risk 1.07, 95\% Cl, 0.98-1.17). Neither was an association found between vessel wall lesion burden and presence of lacunes of presumed vascular origin (adjusted relative risk 1.13,95\% Cl, 0.95-1.34), deep grey matter infarcts (adjusted relative risk 1.25, 95\% Cl, 0.98-1.60 and cortical microinfarcts (adjusted relative risk $1.07,95 \% \mathrm{Cl}, 0.85-1.36)$. When all subcortical infarcts were combined into one composite variable composing the infarcts often considered to be a manifestation of SVD, an association was found between vessel wall lesion burden and presence of an infarct (adjusted relative risk, 1.18; 95\% Cl, 1.03-1.35). In addition, the presence of moderately to severe (Fazekas 2-3) periventricular WMH was associated with vessel wall lesion burden (adjusted relative risk, $1.21 ; 95 \% \mathrm{Cl}$, 1.02-1.42). No association was found between deep WMH and vessel wall lesion burden. An example of the presence of vessel wall lesions and a high WMH score is shown in Figure 2. 


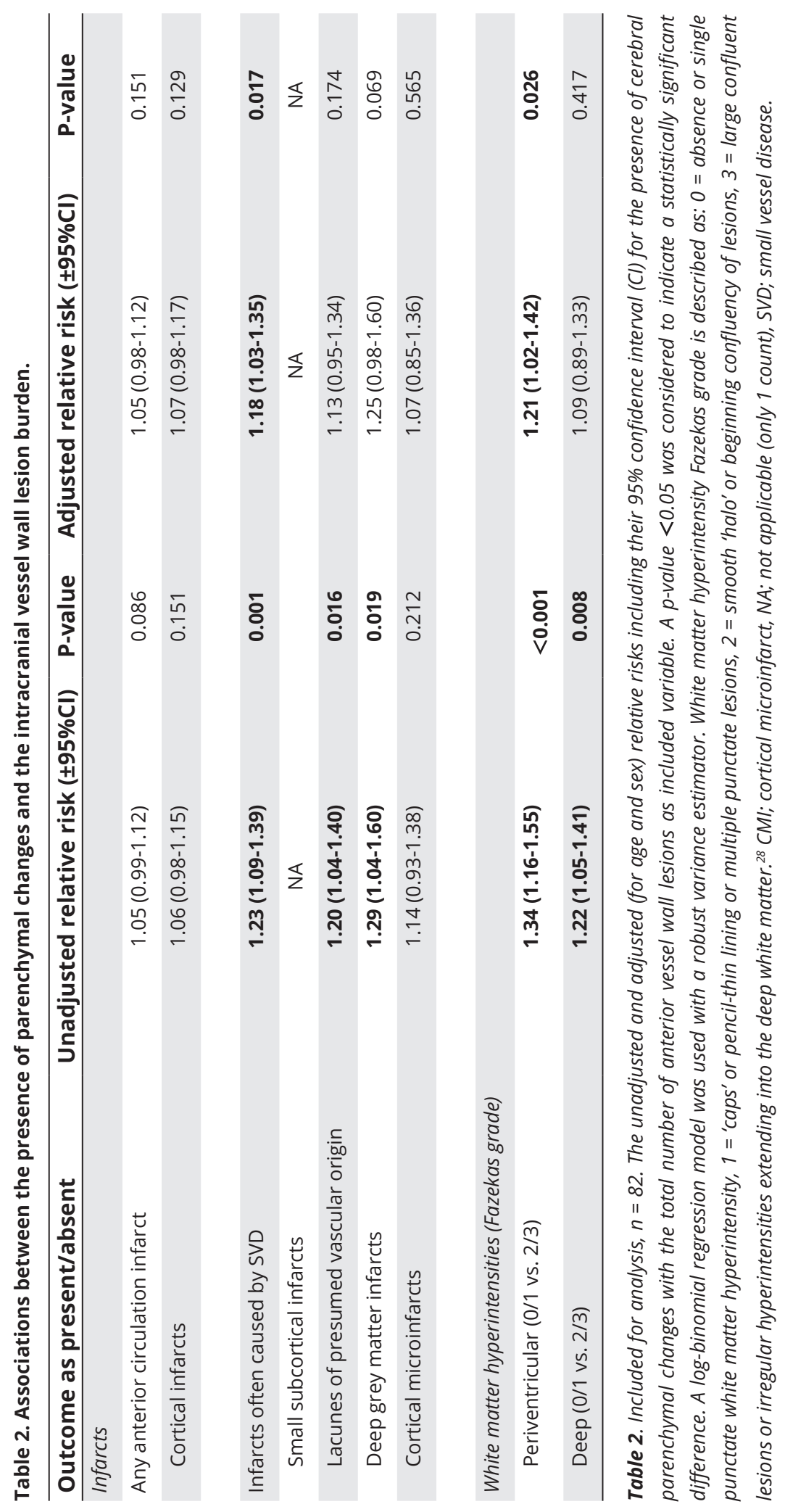



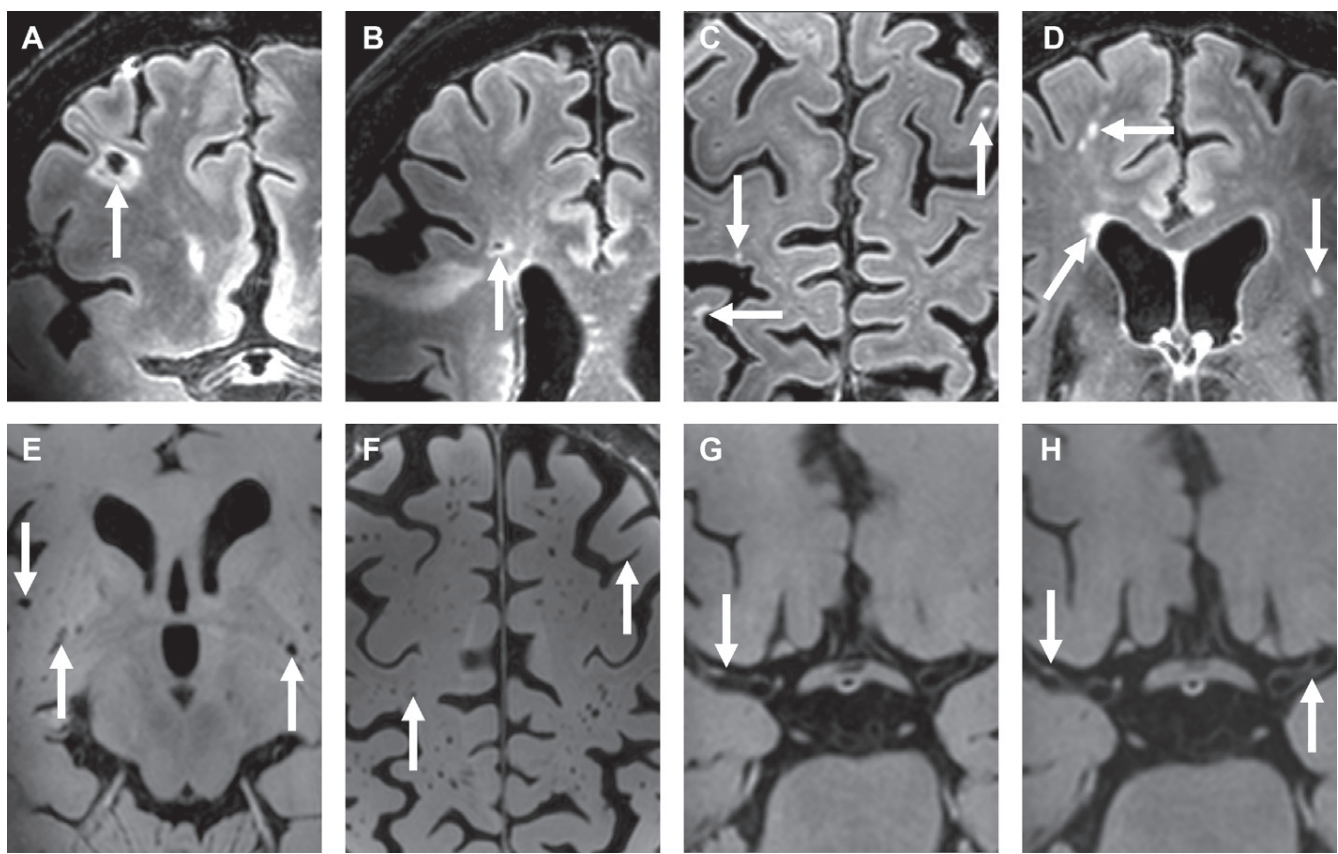

Figure 2: A 72-year-old female with a right-sided ischemic stroke; imaging 25 days after symptom onset. (A-D) Precontrast 7T transverse FLAIR images and (E-H) postcontrast 7T transverse $\mathrm{T}_{1}$-weighted MPIR-TSE vessel wall images. Lacunar infarcts are located in the right frontal (arrow in A) and right frontotemporal area (arrow in B). Multiple cortical microinfarcts are also seen (arrows in C), as well as periventricular and deep white matter hyperintensities (arrows in $\mathbf{D}$ ). Note also the extensive enlarged periventricular spaces in this patient (not scored; arrows in $\mathbf{E}$ and $\mathbf{F}$ ). Vessel wall lesions are seen in the left and right middle cerebral arteries (M1 segments) (arrows in $\mathbf{G}$ and $\mathbf{H}$ ).

In the secondary analyses, we did not find an association between intracranial vessel wall lesion burden and the number of cerebral parenchymal changes of any type (Table 3 ). In the sub analyses, an association was found between the enhancing vessel wall lesion burden and the number of cortical microinfarcts (relative risk, $1.48 ; 95 \% \mathrm{Cl}, 1.04-2.11$ ), but no other association was found between the enhancing vessel wall lesion burden and either presence (Table 4) or number (Table 5) of cerebral parenchymal changes. 


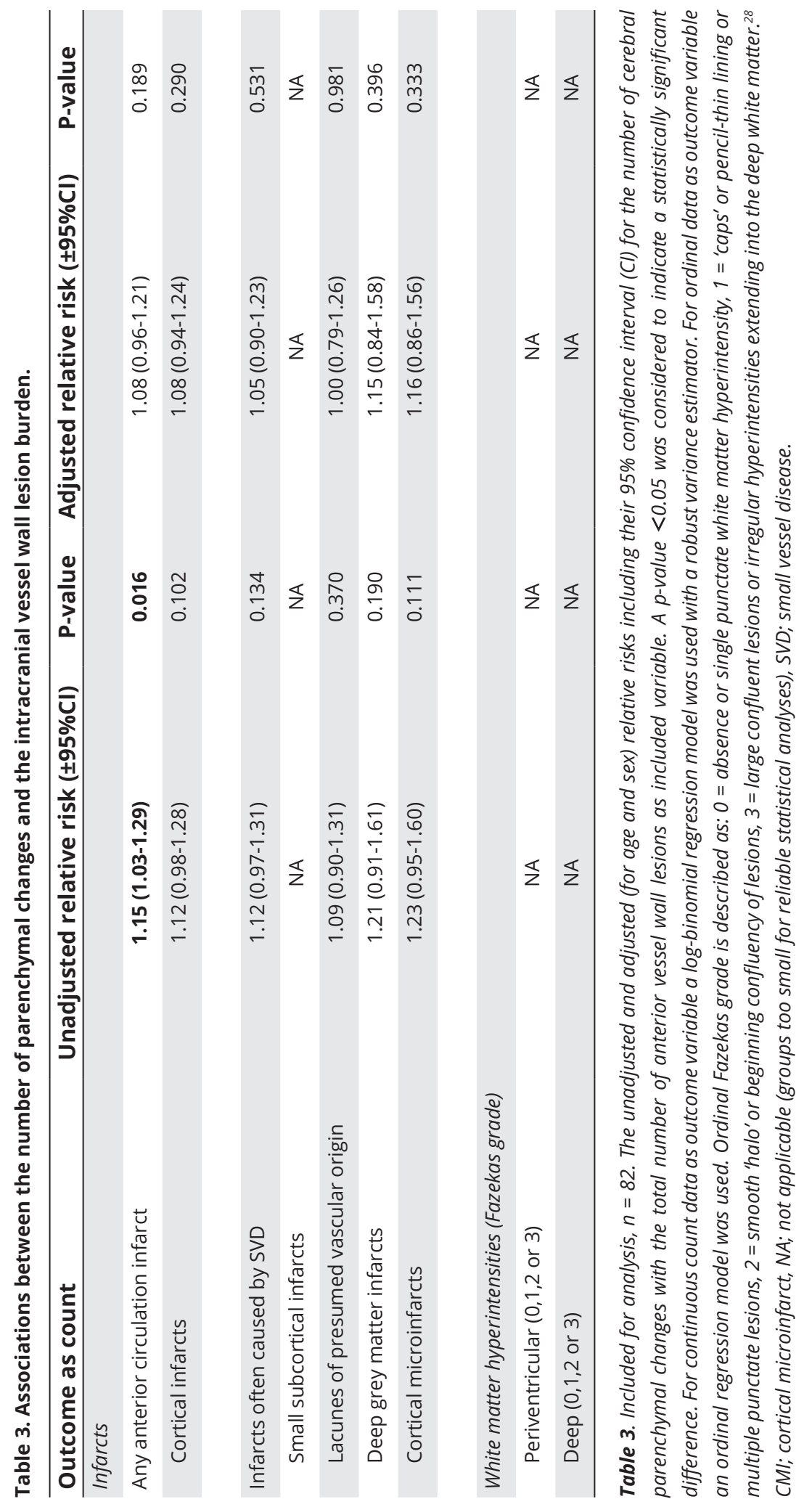




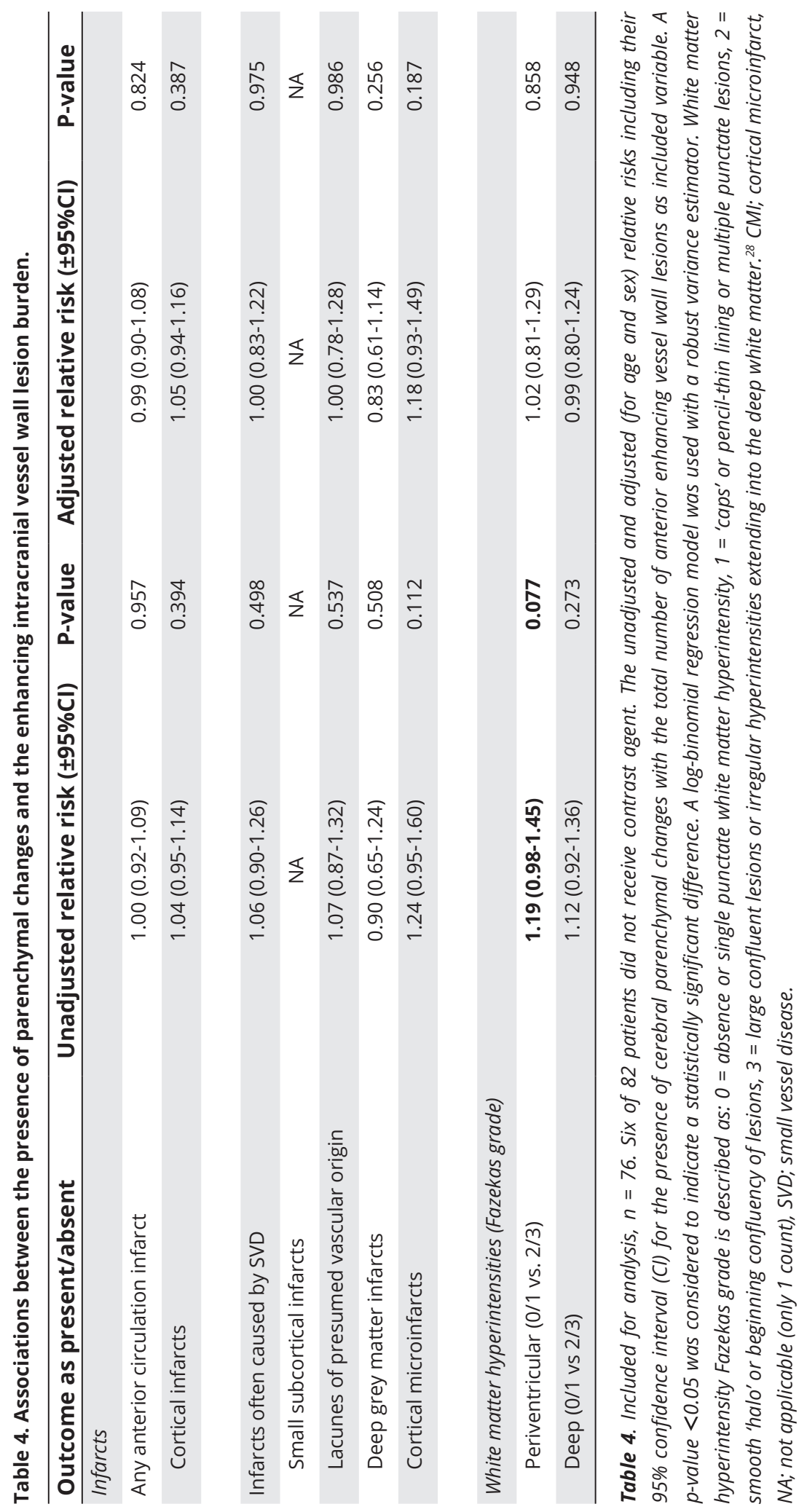




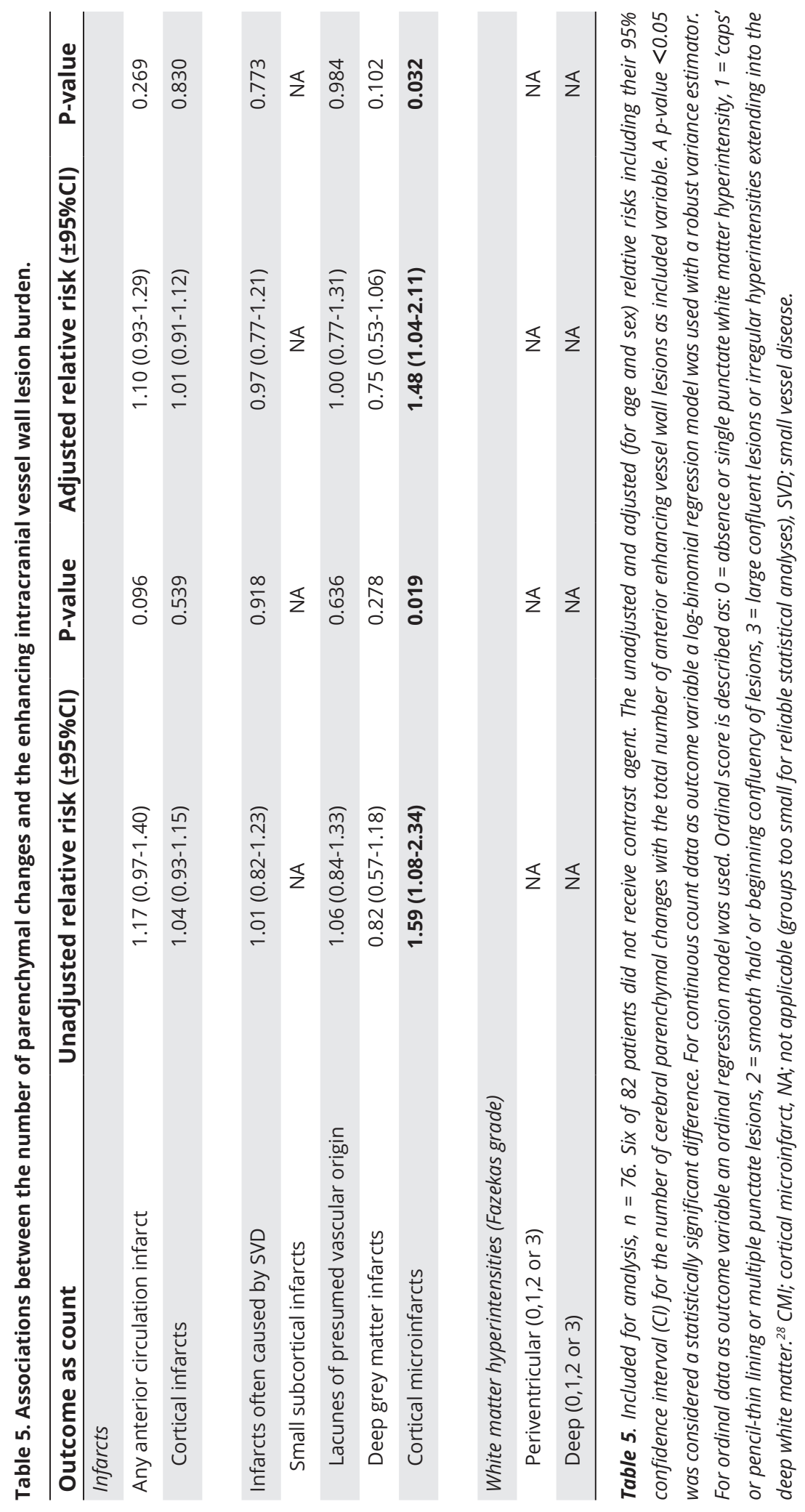




\section{Discussion}

In the current study, an increased vessel wall lesion burden was associated with the presence of either small subcortical infarcts, lacunes of presumed vascular origin or deep grey matter infarcts, and with the presence of moderate to severe periventricular white matter hyperintensities. We did not find an association between intracranial vessel wall lesion burden and presence and number of large cortical infarcts and presence and number of infarcts when classified by type. In addition, the enhancing vessel wall lesion burden was only associated with the number of cortical microinfarcts.

Several of the cerebral parenchymal changes we assessed in our study have been considered manifestations of SVD. ${ }^{27}$ SVD is generally defined by cerebral parenchymal manifestations, as small vessel disease itself is difficult to quantify. ${ }^{19,29-31}$ By contrast, large artery disease can be assessed directly using either lumenography techniques (stenosis) or intracranial vessel wall imaging (vessel wall lesions). ${ }^{9}$ Many studies focus on either large vessel disease or SVD and ongoing effort has been committed to find differences or similarities between cerebral large artery disease and SVD. ${ }^{32-39}$ Despite anatomical and pathophysiological differences between large and small intracranial vessels, the intracranial vasculature is interrelated as one vascular network. SVD has been recently described as a dynamic whole-brain disease that in some cases may share a similar etiology with large vessel disease. In this proposed 'parent artery atheroma theory' atherosclerotic plaques in the large cerebral arteries (e.g., MCA) cause an occlusion at the origin of smaller branching arteries, subsequently leading to cerebral parenchymal changes reflecting SVD. ${ }^{21,39}$ The findings in our study can support this theory as we found an association between the presence of infarcts often related to SVD and the intracranial vessel wall lesion burden (Table 2). Specific microbiota, proteases and immunoglobulins from atherosclerotic plaques can cause a cascade that harms the vessel wall endothelium, the blood brain barrier and consequently could lead to SVD. ${ }^{22}$ This may be an alternative explanation of our findings that the co-occurrence of intracranial vessel wall lesions and parenchymal manifestations of SVD may suggest concomitant changes in both large and small intracranial vasculature.

It remains unclear why an association was found between the enhancing vessel wall lesion burden and number of cortical microinfarcts, but not with presence of cortical microinfarcts nor with presence or number of any other type of infarct. Increasing evidence, including pooled data, show that vessel wall lesion enhancement may be associated with plaque vulnerability and consequently with higher risk of ischemic events..$^{40-42}$ Histopathological validation and especially follow-up studies with a larger sample size are necessary to investigate whether this applies to our study population. 
Several etiologies have been described that attempt to explain different categories of $\mathrm{WMH}$, yet it remains unclear if there indeed exist distinct mechanisms for periventricular and deep WMH development. 19,43,44 The presence of both periventricular and deep WMH may be a result of underlying vascular changes (and subsequent loss of vascular integrity) of both large and small vessel disease. ${ }^{19}$ Nevertheless, different associations between periventricular and deep WMH were found in our study and reasons for these discrepancies have yet to be elucidated. In contrast to previous studies investigating ICAS as a risk factor for (large artery) ischemic stroke, in our study no association was found between intracranial vessel wall lesion burden and presence and number of (large) cortical infarcts. This may be due to the setup of the IVI study, for which specifically ischemic stroke and TIA patients were selected, not limited to large-artery atherosclerosis according to the TOAST criteria, and without inclusion of healthy volunteers. Large numbers of patients are needed to detect small differences in this type of study population. Another explanation (especially for the number of cortical infarcts), may be the difference in definition of ICAS. Previous studies often used intracranial stenosis as a proxy for ICAS, but this represents an advanced state of ICAS. ${ }^{45,46}$ In early to moderate stages of ICAS, vessel wall changes may be its only (early) sign; due to vascular remodeling, luminal narrowing often occurs in advanced stages of the disease. ${ }^{47}$

The strength of this study is that data has been derived from a relatively large number of patients who received $7 \mathrm{~T}$ MR vessel wall imaging; high resolution 7T MRI has been shown superior to $3 T$ MRI in visualizing the intracranial vessel wall because of its high contrast-to-noise ratio, and has a better detection rate of especially small symptomatic and asymptomatic cerebral parenchymal changes..$^{12,48}$ Still, for analyzing all associations in this study, the absolute number of included patients is limited and the inherently induced selection bias due to the design of the study may have affected the analyzed associations underestimating the true association due to reversed causality. Also, the smaller size of the subgroup analysis may have accounted for the lack of an association, which also applies for the analyses with the enhancing vessel wall lesion burden as included variable. When periventricular and deep WMH share the same etiology, this may also be an explanation for the difference in associations between the presence of periventricular versus deep $\mathrm{WMH}$ and vessel wall lesion burden. Overall, there was an overrepresentation of patients with a Fazekas score of 1 in both periventricular and deep $\mathrm{WMH}$, implicating relatively small subgroups of patients with a different Fazekas score. Therefore, (ordinal) regression analyses could not be reliably performed among the individual Fazekas scores. Also, we based our results on multivariable models and tested 7 variables of cerebral parenchymal changes with the presence of (enhancing) vessel wall lesions. The Bonferroni correction for multiple testing would mean that our p-value of 0.05 should be divided by the 9 outcome variables $=0.009$, which indicates statistical 
significance. That approach would leave no association between (enhancing) vessel wall lesions and cerebral parenchymal changes. Furthermore, several other cerebral parenchymal changes have been recognized to reflect (chronic) vascular damage due to SVD, such as cerebral microbleeds, which were not assessed in this study. ${ }^{27}$ Ideally, the inclusion of a larger group of patients could improve the statistical reliability and enable inclusion of more potential confounders or effect modifiers, while assessment of these other cerebral parenchymal changes may contribute to a broader understanding of the potential associations between intracranial vessel wall lesion burden reflecting underlying large artery disease and the cerebral sequelae associated with SVD. Another limitation of assessing intracranial vessel wall lesion burden is the absence of a gold standard method to assess ICAS lesions found on MRI. However, this is a limitation of all studies on intracranial vessel wall MRI, and several post-mortem ex vivo imaging correlation studies have been performed, tentatively increasing the confidence that detected vessel wall lesions represent true intracranial atherosclerosis. ${ }^{15,18,49}$ Yet, the cut-off point between confirmed early stage atherosclerotic lesions and non-pathological or natural variations of vessel wall thickness may be difficult to define.

\section{Conclusions}

Within this relatively small sample size, intracranial vessel wall lesion burden was associated with the presence of periventricular WMH and the presence of any type of infarct often linked to SVD, while no association was found with (large) cortical infarcts. The co-occurrence of intracranial vessel wall lesions and parenchymal manifestations generally attributed to SVD suggest that either vessel wall lesions of the large intracranial parent arteries eventually result in cerebral parenchymal manifestations of SVD, e.g., by occluding the orifices of smaller branching arteries, or that vascular changes occur in both large and small intracranial arteries simultaneously. In the future, studies with larger sample sizes are required to confirm these findings. 


\section{References}

1. Gorelick P, Wong KS, Liu L. Epidemiology. Frontiers of Neurology and Neuroscience 2016;40:34-46.

2. Arenillas JF. Intracranial atherosclerosis: current concepts. Stroke 2011;42:S20-3.

3. Holmstedt CA, Turan TN, Chimowitz MI. Atherosclerotic intracranial arterial stenosis: risk factors, diagnosis, and treatment. The Lancet Neurology 2013;12:1106-14.

4. Qureshi Al, Caplan LR. Intracranial atherosclerosis. Lancet 2014;383:984-98.

5. Bang OY. Intracranial atherosclerosis: current understanding and perspectives. Journal of Stroke 2014;16:27-35.

6. Van der Kolk AG, Zwanenburg JJ, Brundel M, et al. Intracranial vessel wall imaging at 7.0T MRI. Stroke 2011;42:2478-84.

7. Qiao Y, Steinman DA, Qin Q, et al. Intracranial arterial wall imaging using threedimensional high isotropic resolution black blood MRI at 3.0 Tesla. Journal of Magnetic Resonance Imaging: JMRI 2011;34:22-30.

8. Lindenholz A, Harteveld AA, Zwanenburg JJM, Siero JCW, Hendrikse J. Comparison of 3T Intracranial Vessel Wall MRI Sequences. AJNR American Journal of Neuroradioly 2018;39:1112-1120.

9. Mandell DM, Mossa-Basha M, Qiao Y, et al. Intracranial Vessel Wall MRI: Principles and Expert Consensus Recommendations of the American Society of Neuroradiology. AJNR American Journal of Neuroradioly 2017;38:218-229.

10. Bhogal P, Navaei E, Makalanda HL, et al. Intracranial vessel wall MRI. Clinical Radiology 2016;71:293-303.

11. Lindenholz A, van der Kolk AG, Zwanenburg JJM, Hendrikse J. The Use and Pitfalls of Intracranial Vessel Wall Imaging: How We Do It. Radiology 2018;286:12-28.

12. Harteveld AA, van der Kolk AG, van der Worp HB, et al. High-resolution intracranial vessel wall MRI in an elderly asymptomatic population: comparison of 3T and 7T. European Radiology 2017;27:1585-1595.

13. Harteveld AA, Denswil NP, Siero JC, et al. Quantitative Intracranial Atherosclerotic Plaque Characterization at 7T MRI: An Ex Vivo Study with Histologic Validation. AJNR American Journal Neuroradioly 2016;37:802-10.

14. Van der KolkAG, ZwanenburgJJ, Denswil NP, et al. Imaging the intracranial atherosclerotic vessel wall using 7T MRI: initial comparison with histopathology. AJNR American Journal of Neuroradiology 2015;36:694-701.

15. Jiang Y, Zhu C, Peng W, et al. Ex-vivo imaging and plaque type classification of intracranial atherosclerotic plaque using high resolution MRI. Atherosclerosis 2016;249:10-6.

16. Lopez-Cancio E, Dorado L, Millan M, et al. The Barcelona-Asymptomatic Intracranial Atherosclerosis (AsIA) study: prevalence and risk factors. Atherosclerosis 2012;221: 221-5.

17. Qiao Y, Guallar E, Suri FK, et al. MR Imaging Measures of Intracranial Atherosclerosis in a Population-based Study. Radiology 2016:280:860-8. 
18. Yang WJ, Wong KS, Chen XY. Intracranial Atherosclerosis: From Microscopy to HighResolution Magnetic Resonance Imaging. Journal of Stroke 2017;19:249-60.

19. Wardlaw JM, Smith C, Dichgans M. Mechanisms of sporadic cerebral small vessel disease: insights from neuroimaging. The Lancet Neurology 2013;12:483-97.

20. Van Veluw SJ, Shih AY, Smith EE, et al. Detection, risk factors, and functional consequences of cerebral microinfarcts. Lancet Neurology 2017;16:730-40.

21. Shi Y, Wardlaw JM. Update on cerebral small vessel disease: a dynamic whole-brain disease. Stroke and Vascular Neurology 2016;1:83-92.

22. Ihara M, Yamamoto Y. Emerging Evidence for Pathogenesis of Sporadic Cerebral Small Vessel Disease. Stroke 2016:47:554-60.

23. Van der Kolk AG, Hendrikse J, Brundel M, Biessels GJ, Smit EJ, Visser F. Multi-sequence whole-brain intracranial vessel wall imaging at 7.0 tesla. European Radiology 2013;23:2996-3004.

24. Adams HP, Jr., Bendixen BH, Kappelle LJ, et al. Classification of subtype of acute ischemic stroke. Definitions for use in a multicenter clinical trial. TOAST. Trial of Org 10172 in Acute Stroke Treatment. Stroke 1993;24:35-41.

25. Lindenholz A, van der Kolk AG, van der Schaaf IC, et al. Intracranial Atherosclerosis Assessed with 7-T MRI: Evaluation of Patients with Ischemic Stroke or Transient Ischemic Attack. Radiology 2020;295:162-70.

26. Klein S, Staring M, Murphy K, Viergever MA, Pluim JP. elastix: a toolbox for intensity-based medical image registration. IEEE Transactions on Medical Imaging 2010;29:196-205.

27. Wardlaw JM, Smith EE, Biessels GJ, et al. Neuroimaging standards for research into small vessel disease and its contribution to ageing and neurodegeneration. The Lancet Neurology 2013;12:822-38.

28. Fazekas F, Chawluk JB, Alavi A, Hurtig HI, Zimmerman RA. MR signal abnormalities at 1.5 $T$ in Alzheimer's dementia and normal aging. AJR American Journal of Roentgenology 1987;149:351-6.

29. Wardlaw JM, Dennis MS, Warlow CP, Sandercock PA. Imaging appearance of the symptomatic perforating artery in patients with lacunar infarction: occlusion or other vascular pathology? Annals of Neurology 2001;50:208-15.

30. Pantoni L. Cerebral small vessel disease: from pathogenesis and clinical characteristics to therapeutic challenges. The Lancet Neurology 2010;9:689-701.

31. Harteveld AA, De Cocker LJ, Dieleman N, et al. High-resolution postcontrast time-of-flight MR angiography of intracranial perforators at 7.0 Tesla. PLoS One 2015;10:e0121051.

32. Kwon HM, Lynn MJ, Turan TN, et al. Frequency, Risk Factors, and Outcome of Coexistent Small Vessel Disease and Intracranial Arterial Stenosis: Results From the Stenting and Aggressive Medical Management for Preventing Recurrent Stroke in Intracranial Stenosis (SAMMPRIS) Trial. JAMA Neurology 2016;73:36-42.

33. Bang OY, Chung JW, Ryoo S, et al. Brain microangiopathy and macroangiopathy share common risk factors and biomarkers. Atherosclerosis 2016;246:71-7. 
34. Mead GE, Lewis SC, Wardlaw JM, Dennis MS, Warlow CP. Severe ipsilateral carotid stenosis and middle cerebral artery disease in lacunar ischaemic stroke: innocent bystanders? Journal of Neurology 2002;249:266-71.

35. Brisset $M$, Boutouyrie $P$, Pico $F$, et al. Large-vessel correlates of cerebral small-vessel disease. Neurology 2013;80:662-9.

36. Aboyans V, Lacroix $\mathrm{P}$, Criqui $\mathrm{MH}$. Large and small vessels atherosclerosis: similarities and differences. Progress in Cardiovascular Diseases 2007;50:112-25.

37. Bang OY, Joo SY, Lee PH, et al. The course of patients with lacunar infarcts and a parent arterial lesion: similarities to large artery vs small artery disease. Archives of Neurology 2004;61:514-9.

38. Dieleman N, van der Kolk AG, Zwanenburg JJ, et al. Relations between location and type of intracranial atherosclerosis and parenchymal damage. Journal of Cerebral Blood Flow Metabolism: JCBFM 2016;36:1271-80.

39. Kong Q, Zhang Z, Yang Q, et al. 7T TOF-MRA shows modulated orifices of lenticulostriate arteries associated with atherosclerotic plaques in patients with lacunar infarcts. European Journal of Radiology 2019;118:271-6.

40. Qiao Y, Zeiler SR, Mirbagheri S, Leigh R, Urrutia V, Wityk R. Intracranial plaque enhancement in patients with cerebrovascular events on high-spatial-resolution MR images. Radiology 2014;271.

41. Wang Y, Liu X, Wu X, Degnan AJ, Malhotra A, Zhu C. Culprit intracranial plaque without substantial stenosis in acute ischemic stroke on vessel wall MRI: A systematic review. Atherosclerosis 2019;287:112-21.

42. Lee HN, Ryu CW, Yun SJ. Vessel-Wall Magnetic Resonance Imaging of Intracranial Atherosclerotic Plaque and Ischemic Stroke: A Systematic Review and Meta-Analysis. Frontiers in Neurology 2018;9:1032.

43. Kim KW, MacFall JR, Payne ME. Classification of white matter lesions on magnetic resonance imaging in elderly persons. Biological Psychiatry 2008;64:273-80.

44. Rost NS, Rahman RM, Biffi A, et al. White matter hyperintensity volume is increased in small vessel stroke subtypes. Neurology 2010;75:1670-7.

45. Ritz K, Denswil NP, Stam OC, van Lieshout J, Daemen MJ. Cause and mechanisms of intracranial atherosclerosis. Circulation 2014;130:1407-14.

46. Arenillas JF, Lopez-Cancio E, Wong KS. Biomarkers, Natural Course and Prognosis. Frontiers of Neurology and Neuroscience 2016;40:93-108.

47. Qiao Y, Anwar Z, Intrapiromkul J, et al. Patterns and Implications of Intracranial Arterial Remodeling in Stroke Patients. Stroke 2016;47:434-40.

48. Zhu $\mathrm{C}$, Haraldsson $\mathrm{H}$, Tian $\mathrm{B}$, et al. High resolution imaging of the intracranial vessel wall at 3 and 7 T using 3D fast spin echo MRI. MAGMA 2016;29:559-70.

49. Harteveld AA, Denswil NP, Siero JC, et al. Quantitative Intracranial Atherosclerotic Plaque Characterization at 7T MRI: An Ex Vivo Study with Histologic Validation. AJNR American Journal of Neuroradiology 2016:37:802-10. 


\section{Supplemental Material}

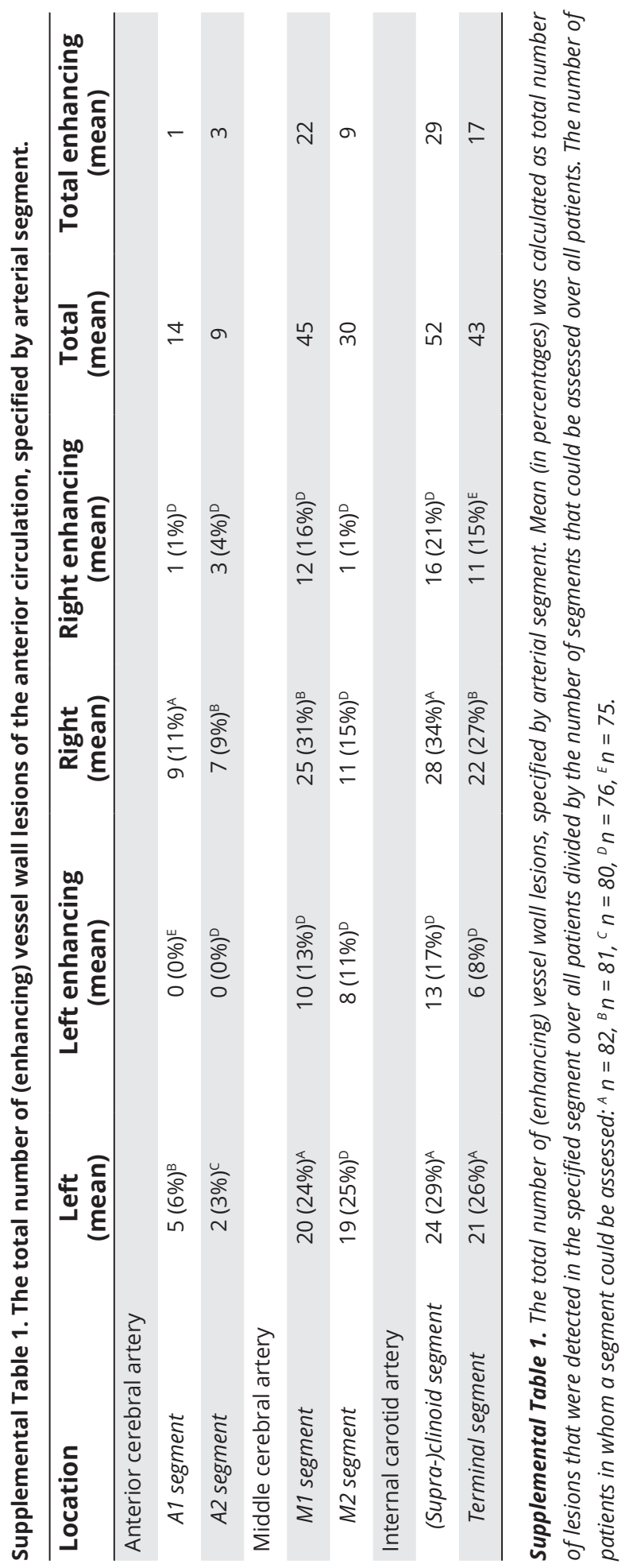




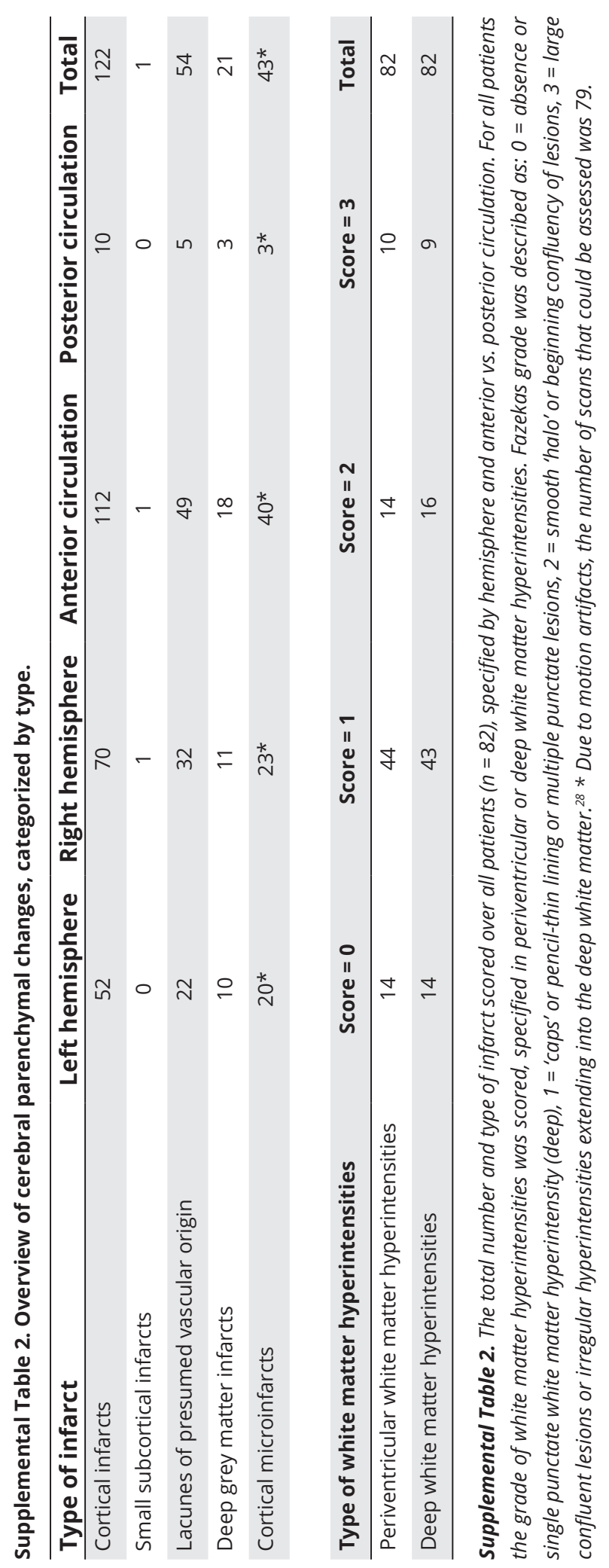


Intracranial Atherosclerotic Burden and Cerebral Parenchymal Changes at 7T MRI 


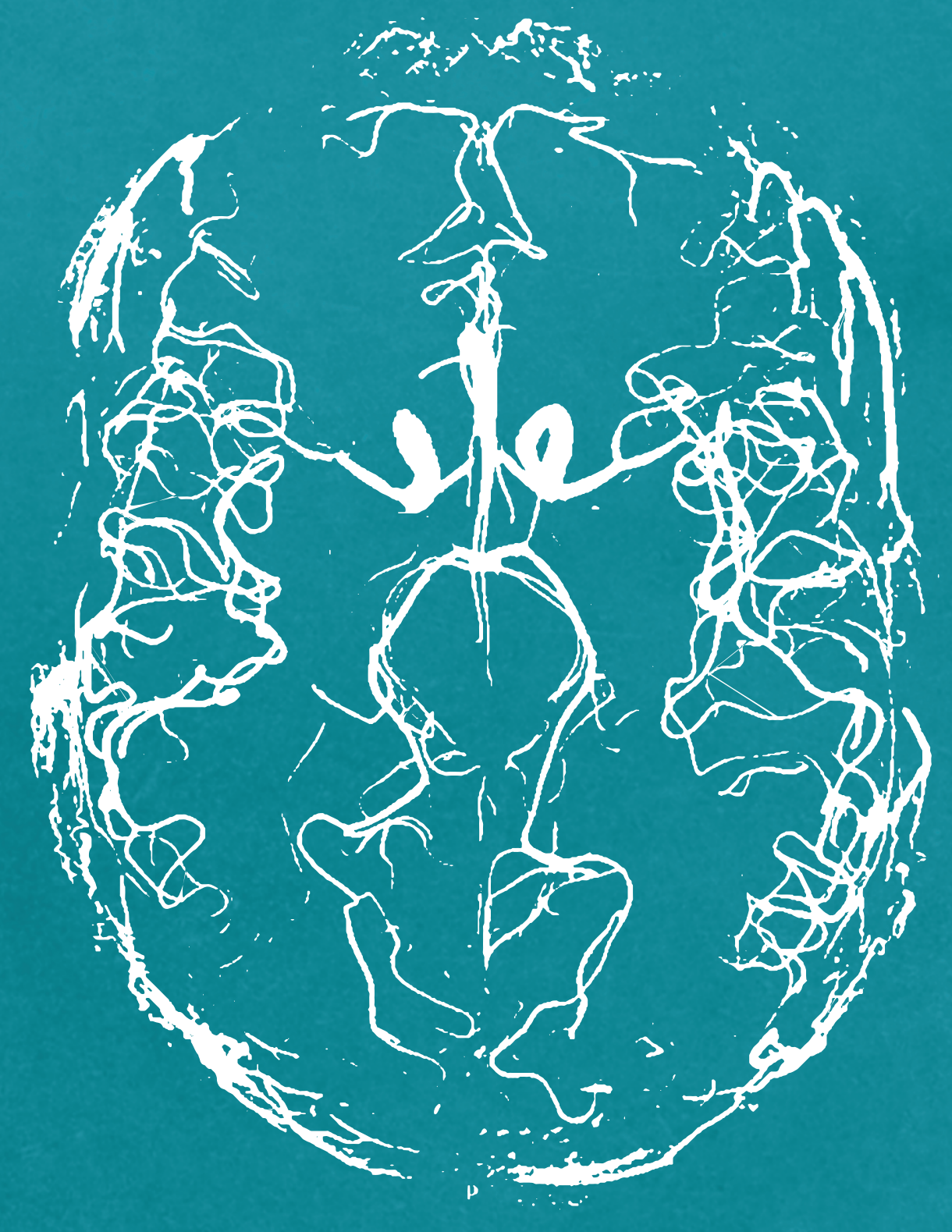




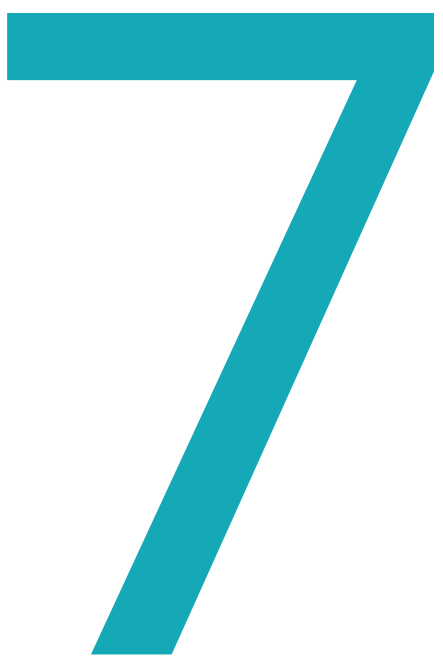

\section{MRI Vessel Wall Imaging after Intra-Arterial Treatment for Acute Ischemic Stroke}

Arjen Lindenholz' ${ }^{1}$ Irene C. van der Schaaf', Anja G. van der Kolk' ${ }^{1}$, H. Bart van der Worp ${ }^{2}$, Anita A. Harteveld ${ }^{1}$, L. Jaap Kappelle ${ }^{2}$ and Jeroen Hendrikse ${ }^{1}$

${ }^{1}$ Department of Radiology, University Medical Center Utrecht, The Netherlands ${ }^{2}$ Department of Neurology and Neurosurgery, University Medical Center Utrecht, Brain Center Rudolf Magnus, The Netherlands 


\begin{abstract}
Background and purpose: Vessel wall MR imaging is increasingly performed in the diagnostic work-up of patients with ischemic stroke. The aim of this study was to compare vessel wall enhancement after intra-arterial thrombosuction with that in patients not treated with thrombosuction.
\end{abstract}

Materials and methods: From 2009 to 2017, forty-nine patients with an ischemic stroke underwent 7T MR imaging within 3 months after symptom onset as part of a prospective intracranial vessel wall MR imaging study. Fourteen of these patients underwent intra-arterial treatment (IAT) using thrombosuction (IAT-group). In the IAT-group, vessel walls were evaluated for major vessel wall changes. All patients underwent pre- and postcontrast vessel wall MR imaging to assess enhancing foci of the vessel wall using coregistered subtraction images. A Wilcoxon signed-rank test was performed to test for differences.

Results: In the IAT-group 11 of 14 patients (79\%) showed vessel wall enhancement compared with 17 of 35 without intra-arterial treatment (49\%). In the IAT-group, more enhancing foci were detected on the ipsilateral side $(n=18.5)$ compared with the contralateral side $(n=3, p=0.005)$. Enhancement was more often concentric on the ipsilateral side $(n=8)$ compared with contralateral side $(n=0, \mathrm{p}$ $=0.01$ ). No differences were found in the non-IAT group between the number and configuration of ipsilateral and contralateral enhancing foci.

Conclusion: Patients treated with intra-arterial treatment by means of thrombosuction showed more (concentric) enhancing foci of the vessel wall ipsilateral compared with contralateral to the treated artery than the patients without intra-arterial treatment, suggesting reactive changes of the vessel wall. This should be taken into account when assessing vessel wall MR images in patients with stroke. 


\section{Introduction}

Intra-arterial treatment (IAT) has proved beneficial in selected patients with an anterior circulation acute ischemic stroke. ${ }^{1}$ With IAT, revascularization of the occluded artery by means of mechanical thrombectomy can be achieved in most patients, restoring blood flow to the brain tissue. Although the overall effects of IAT on clinical outcome are well-known, ${ }^{1,2}$ not much is known about the effects of thrombectomy on the local intracranial vessel wall. Recently, there has been concern that IAT might damage the arterial vessel wall.3-6

Thrombectomy can be performed with different devices: Stent-retrievers or thrombosuction devices have been used most often. ${ }^{2}$ Damage to the intracranial vessel wall may be caused by (repeated) mechanical sheer stress of the stentretriever on the vessel wall, ${ }^{4}$ or the negative pressure (up to $-50 \mathrm{~cm} / \mathrm{Hg}$ ) when using a thrombosuction device. ${ }^{5,7}$ This damage may consist of dissections, vessel wall edema, and rupture or damage to the endothelium, potentially leading to recurrent thrombosis and distal embolism. ${ }^{3-6}$ Histopathological preclinical studies have shown endothelial damage to the vessel wall after thrombectomy, more evident with stent-retriever devices than with thrombosuction devices. ${ }^{3-5,8-10}$ In recent MR imaging studies and other imaging studies performed after IAT, damage of a major vessel wall such as dissection or stenosis was only rarely reported..$^{5,8-12}$ However, the arterial vessel wall more often showed contrast enhancement or wall thickening than arteries of patients who did not undergo IAT..$^{11-13}$ In these series, patients were treated mostly with stent-retrievers and scanning was performed with 3 T MR imaging scanners in the acute setting (first day to first week) after IAT. The longer-term effects after IAT performed by thrombosuction have not been reported yet.

Intracranial vessel wall MRI is expected to be increasingly performed in the diagnostic work-up and follow-up of patients with stroke. The aim of this study was to assess the frequency of longer-term vessel wall changes - by assessing the presence and severity of intracranial vessel wall enhancement - in patients with stroke after IAT with thrombosuction and compare them with patients with stroke without IAT. Potential differences may support the diagnostic interpretation of intracranial vessel wall changes after acute stroke treatment. 


\section{Materials and Methods}

\section{Study population}

From December 2009 to November 2017, patients with an acute ischemic stroke in the anterior circulation who were treated at the neurology department of the University Medical Center Utrecht were screened for inclusion. Patients were retrieved from the ongoing Intracranial Vessel wall Imaging (IVI) study (NTR2119, www.trialregister.nl), a prospective vessel wall MRI study recruiting patients who presented with clinical symptoms of anterior circulation ischemia (transient ischemic attack (TIA) or stroke). ${ }^{14}$ Main inclusion criteria for the current study were age older than 18 years and the possibility of undergoing a 7T MRI examination within three months after symptom onset. The patients without IAT were selected based on an anterior circulation non-lacunar infarct. Patients with contraindications for MR imaging or for gadolinium-containing contrast agents were excluded, as well as patients with ischemic stroke caused by vasculitis, reversible cerebral vasoconstriction syndrome, small vessel disease or secondary to a recent surgical or interventional procedure. Additional exclusion criteria for the current study were primary treatment with a different strategy than a thrombosuction device for the IAT-group to improve study population homogeneity, and previous IAT or TIA as final diagnosis for the non-IAT group. Findings of 23 patients without IAT have been published before.15,16 These prior articles dealt with sequence development and vessel wall lesion prevalence whereas in this study we report longer-term intracranial vessel wall enhancement after IAT using thrombosuction compared with patients not treated with IAT. This study was approved by the Institutional Review Board of our hospital and all patients gave written informed consent. For all patients, baseline characteristics including age, sex, vascular risk factors, stroke severity expressed using the National Institutes of Health Stroke Scale (NIHSS) as well as stroke classification and time intervals between IAT and imaging were collected. For the IAT-group, time intervals between symptom onset and treatment, procedural time, number of passes needed for thrombus removal, and concomitant treatment with intravenous (IV) recombinant tissuetype plasminogen activator (alteplase), were additionally collected.

\section{Treatment}

Treatment was performed as part of standard clinical care. All patients who were eligible for intravenous thrombolysis received IV alteplase within the 4.5-hour time window from symptom onset. IAT was introduced in our center during the study period, after the international IAT trial results, and the first patient treated with a thrombosuction device was included in 2014. The main criteria for IAT were derived from the Multicenter Randomized Clinical Trial of Endovascular Treatment for Acute Ischemic Stroke in The Netherlands (MR Clean) trial: ${ }^{1} 1$. A clinical diagnosis of 
acute ischemic stroke caused by an intracranial anterior circulation occlusion that was visible on computed tomographic angiography (CTA), MR angiography (MRA) or digital subtraction angiography (DSA); and 2. Treatment could be performed within six hours from symptom onset. All patients in the IAT-group were treated under general anesthesia. A thrombosuction device (Penumbra, Inc., Alameda, CA) was used in all patients included in this study. Procedural complications involving the vessel wall, including dissection or perforation, were noted.

\section{Imaging}

Imaging was performed on a 7T whole-body MR system (Philips Healthcare, Best, The Netherlands) with either a 16-channel or a 32-channel receive coil and a volume transmit/receive coil for transmission (Nova Medical, Wilmington, MA, USA). Vessel wall visualization at 7T MRI has been shown to be superior compared with $3 T$ MRI because of a higher contrast-to-noise ratio and image quality. ${ }^{17,18}$ The imaging protocol included a dedicated pre- and postcontrast 3D wholebrain $\mathrm{T}_{1}$-weighted magnetization-prepared inversion recovery turbo spin-echo (MPIR-TSE) vessel wall sequence and a postcontrast time-of-flight (TOF) MRA. ${ }^{16}$ For the postcontrast image acquisitions a gadolinium-containing contrast agent (gadobutrol, Gadovist $1.0 \mathrm{mmol} / \mathrm{mL}$, Bayer Schering Pharma, Newbury, UK), doseadjusted to patient weight, was administered intravenously. The TOF-MRA images were used for anatomical verification of the vessels seen on the MPIR-TSE images. The following scan parameters were used for the MPIR-TSE sequence: field-ofview (FOV) $220 \times 180 \times 13 \mathrm{~mm}^{3}$, which was optimized to $250 \times 250 \times 190 \mathrm{~mm}^{3}$ and satisfactorily tested for equality in vessel wall lesion detection during the study period, ${ }^{16}$ acquired spatial resolution $0.8 \times 0.8 \times 0.8 \mathrm{~mm}^{3}$, reconstructed spatial resolution $0.49 \times 0.49 \times 0.49 \mathrm{~mm}^{3}$, repetition time (TR) $3952 \mathrm{~ms}$, echo time (TE) 37 ms, inversion time ( $\mathrm{TI}) 1375 \mathrm{~ms}$, flip-angle (FA) 120 degrees, readout bandwidth $935 \mathrm{~Hz}$, and an acquisition time of 10 minutes 40 seconds. For the small FOV sequence, the FOV was placed so that the distal intracranial carotid artery and middle cerebral artery were included in the FOV. Scan parameters for the TOFMRA were as follows: FOV $190 \times 190 \times 102 \mathrm{~mm}^{3}$, acquired spatial resolution $0.4 \times$ $0.5 \times 0.6 \mathrm{~mm}^{3}$, reconstructed spatial resolution $0.4 \times 0.4 \times 0.3 \mathrm{~mm}^{3}$, TR $21 \mathrm{~ms}$, TE $2.3 \mathrm{~ms}$, FA 30 degrees, readout bandwidth $557 \mathrm{~Hz}$, and an acquisition time of 9 minutes 18 seconds.

\section{Image assessment}

Image assessment was performed offline on a PACS (Picture Archiving and Communication System; IDS7, Sectra Medical Systems, Sectra AB, Sweden). All images were independently assessed by two readers with expertise in reading neurovascular vessel wall images (AK, 9 years of experience and $A L, 4$ years of experience). Readers were blinded for any patient characteristics. The arterial 
segments that were analyzed included the left and right intracranial internal carotid artery (ICA, the clinoid, supra-clinoid and terminal segments) and the left and right middle cerebral artery (MCA, M1 and M2 segments). Recanalization after IAT was assessed with $\mathrm{TICl}$-grading on post-procedural DSA images. ${ }^{19}$

First, in the IAT-group post-procedural DSA, 7T MR vessel wall, and 7T MRA images were assessed for dissections and stenoses as major vessel wall changes. Intracranial stenoses were classified into $<50 \%$ stenosis (minor), $50-69 \%$ stenosis (moderate), $70-99 \%$ stenosis (severe), and occlusion. ${ }^{20}$ Second, all 7T MR vessel wall images were assessed for the presence and number of enhancing foci per arterial segment. The mean numbers of enhancing foci of both readers were used for the analyses. Assessment was blinded to IAT and non-IAT. In all patients (IAT-group and non-IAT group) the arteries in the hemisphere ipsilateral to the ischemic infarction were compared with those of the contralateral side. All enhancing foci were further classified as either concentric (circumferential of the vessel wall $>50 \%$ enhancing) or eccentric ( $<50 \%$ circumference enhancement) type of enhancement. Intracranial atherosclerosis more often shows eccentric vessel wall enhancement and an inflammatory state of the vessel wall show most often concentric vessel wall enhancement. ${ }^{21,22}$

The method and cut-off point for the configuration of vessel wall lesion assessment by visual inspection has been described before as a clinically usable tool for vessel wall assessment. ${ }^{21}\{$ Lindenholz, 2018 \#3944\} In the assessment of contrast enhancement, a focus was considered as enhancing when the signal intensity approximated the signal intensity of the (enhancing) pituitary stalk and was present in at least two slices. Next, pre- and postcontrast vessel wall images were compared side-by-side to confirm the enhancement. As a double confirmation of enhancement subtraction images were calculated and used. For this purpose, pre- and postcontrast vessel wall images were coregistered for the whole 3D volume using the elastix toolbox in MeVisLab (version 2.7; MeVis Medical Solutions, Bremen, Germany). ${ }^{23}$ Subsequently, precontrast vessel wall images were subtracted from the co-registered postcontrast vessel wall images and assessed for contrast enhancement. The registration parameters, $\Delta$ Rotation (in degrees) and $\Delta$ Translation (in millimeters), were used as a measure of motion between pre- and postcontrast vessel wall sequences. The $\Delta$ Rotation and $\Delta$ Translation parameters were calculated as $\sqrt{ }\left(X\right.$-axis ${ }^{2}+Y$-axis ${ }^{2}+Z$-axis $\left.{ }^{2}\right)$. When more than one enhancing focus was detected within one arterial segment, they were counted separately when they were separated from each other by a normal appearing vessel wall segment in between. Also, enhancing foci at the location where the ICA crosses the dura mater from extracranial to intracranial, suspicious for vasa vasorum, were not considered as vessel wall enhancement. 


\section{Statistical analysis}

SPSS version 21.0 for Windows (IBM, Armonk, New York) was used for statistical analysis. Descriptive statistics were used for the frequency of intracranial dissection or stenosis. Counts are given in proportions (percentages) including their 95\% confidence intervals (CI). The intraclass correlation coefficient (ICC) using a twoway mixed, average measurement, consistency model and the Dice similarity coefficient (DSC) to correct for the location of the enhancement were calculated to evaluate the interrater agreement. A Wilcoxon signed-rank test was used for comparison between the number of enhancing foci ipsilateral and contralateral to the ischemic site as seen on the vessel wall images. A Mann-Whitney $U$ test was performed to compare the number of enhancing foci between the IAT-group and non-IAT group. A two-sided p-value $<0.05$ was considered statistically significant.

\section{Results}

\section{Patients and treatment}

Between December 2009 and October 2017, forty-nine patients were included in this study. A flowchart of the study inclusion is shown in Figure 1. Baseline characteristics for both patient groups can be found in the Table 1.

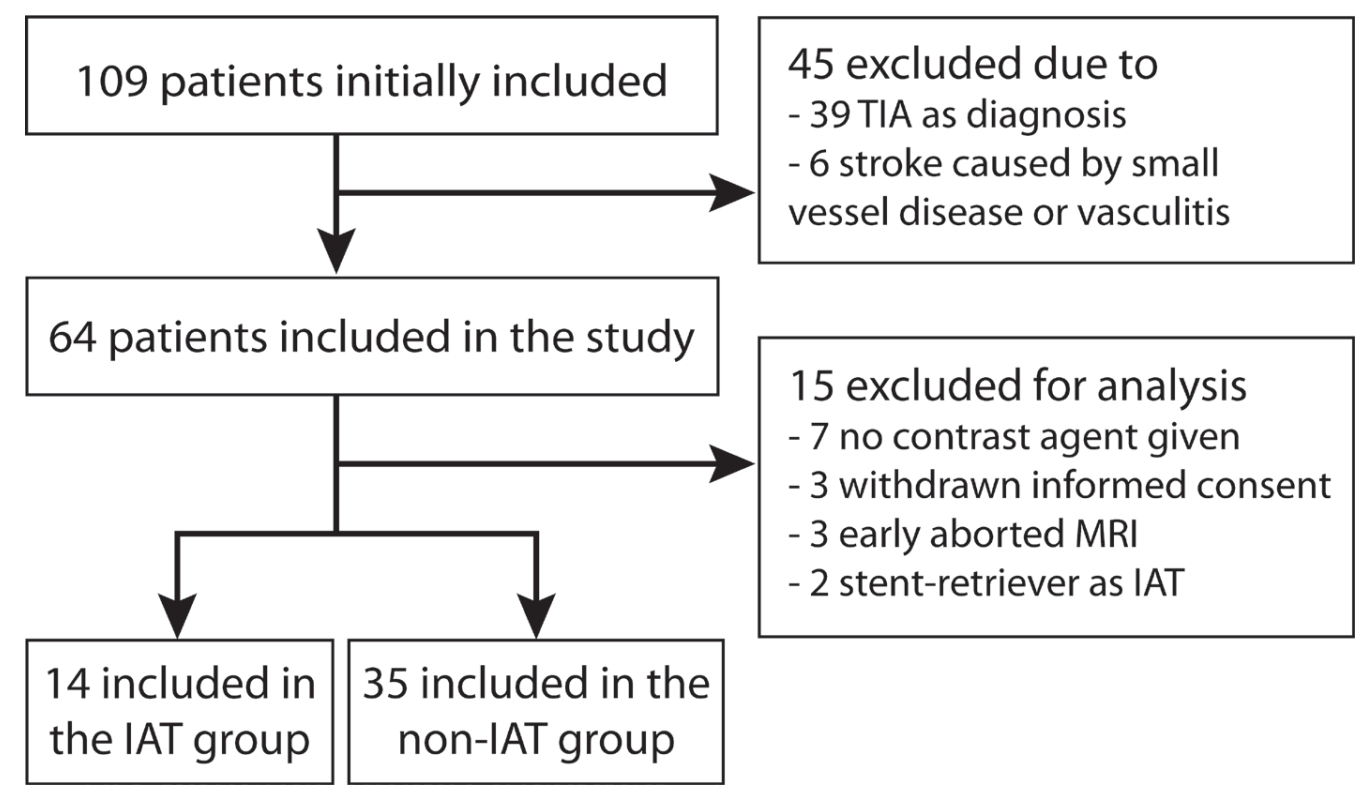

Figure 1. Flowchart of study inclusion. IAT: intra-arterial treatment. 


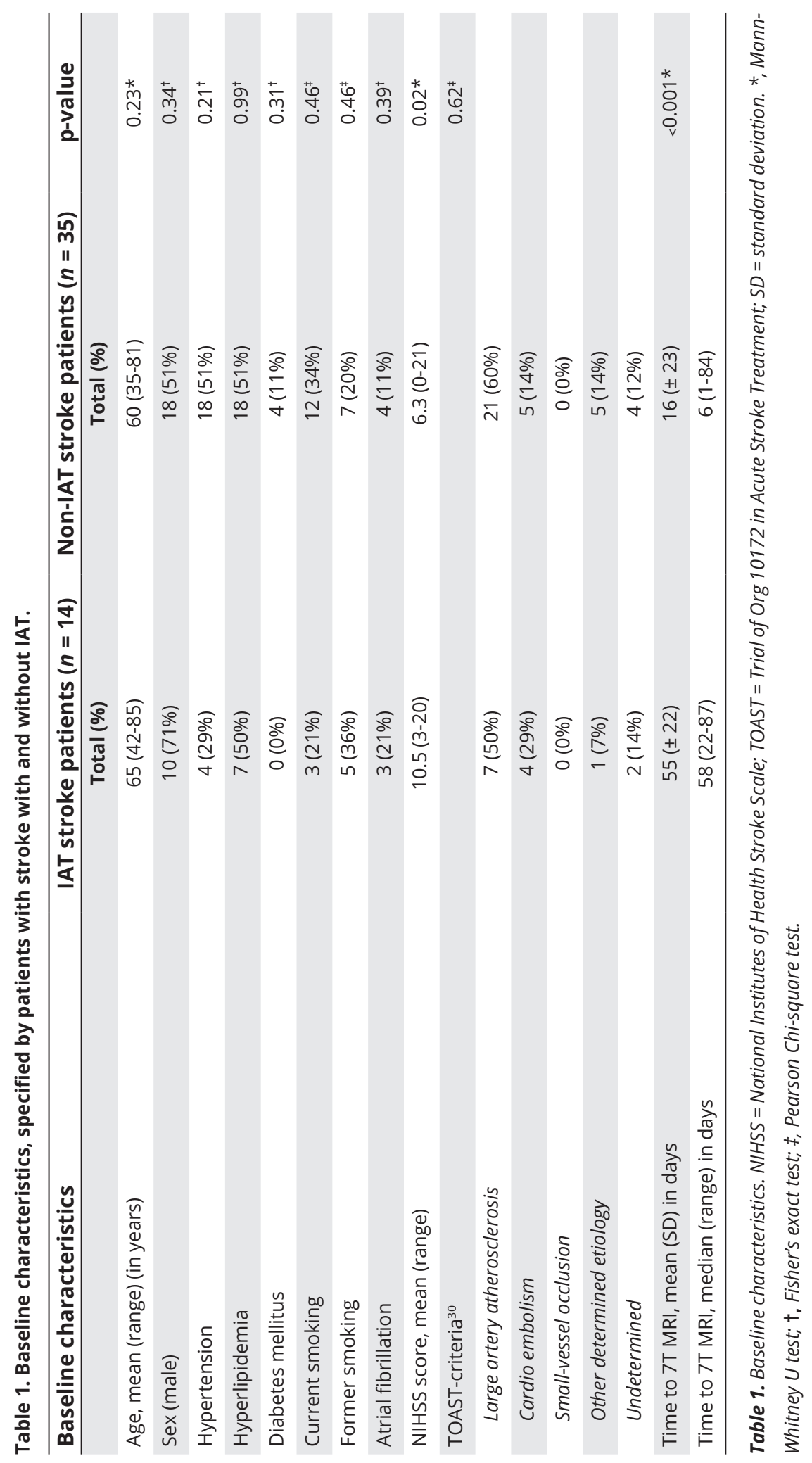


MRI Vessel Wall Imaging after Intra-Arterial Treatment for Acute Ischemic Stroke

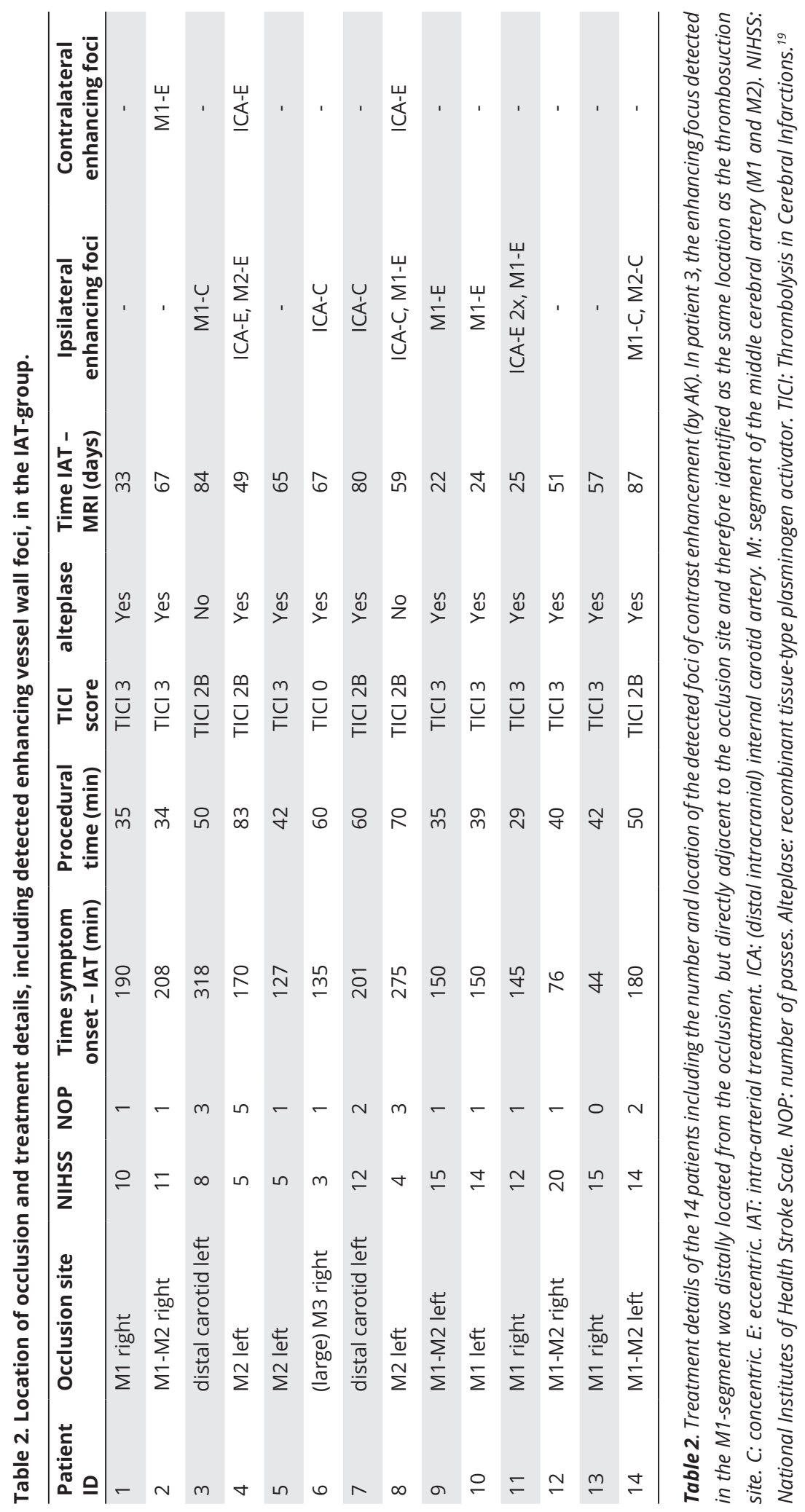


Fourteen patients underwent IAT using thrombosuction; two of them had an occlusion in the distal intracranial ICA and 12 patients in the MCA (Table 2). Twelve patients received intravenous alteplase prior to intra-arterial mechanical thrombectomy; the other 2 patients exceeded the 4.5-hour time window for alteplase treatment. In two patients a stent-retriever (Solitaire, Covidien, Irvine, California; or Trevo, Stryker, Kalamazoo, Michigan) was used in addition to the thrombosuction device (patients 4 and 14, Table 2). IAT was technically successful in 13 of 14 patients. No procedure-related hemorrhagic complications occurred during the procedures in any of the patients. In 9 patients one pass was sufficient; in the remaining 5 patients more passes were needed (range 2-5) to remove the culprit thrombus.

In the non-IAT group, 5 of 35 patients had an ICA infarction (right-sided, $n=3$ ) and 30 patients an MCA infarction (right-sided, $n=16$ ) (Supplemental table 1). Twelve patients received intravenous alteplase as standard ischemic stroke treatment. In the remaining 23 patients, the 4.5-hour time window was exceeded or only minor symptoms were still present on admission.

\section{Vessel wall assessment}

Major vessel wall changes in the IAT-group

Besides a post-procedural moderate stenosis in the treated artery of one patient, no other major vessel wall changes were detected. This stenosis in the proximal left M2-segment was detected by MR vessel wall imaging and TOF-MRA ( $>6$ week after IAT) at the same location as the removed thrombus and was not visible yet on the post-procedural angiogram performed within the same session as the thrombectomy. It is not clear whether this was caused by an atherosclerotic plaque or restenosis or was treatment-induced. In patients with a middle cerebral artery occlusion no $T_{1}$-hyperintensity before contrast was found, nor did we find an MRI vessel wall indication suggesting an intramural hemorrhage.

\section{Vessel wall enhancement}

The interrater agreement for the number and location of the enhancing foci was an ICC of $0.91(95 \% \mathrm{Cl} ; 0.84-0.95)$ and a DSC of 0.87. Examples of foci of vessel wall enhancement at the thrombectomy site are shown in Figure 2, Figure 3 and Figure 4. An example of vessel wall enhancement of the non-IAT group is shown in Figure 5. In the IAT-group 11 of 14 patients (79\%) showed vessel wall enhancement compared with 17 of 35 patients (49\%) in the non-IAT group. As an average of both readers, in total, 21.5 foci of vessel wall enhancement were detected in the 84 vessel segments (26\%) of the IAT patients compared with 30 foci of vessel wall enhancement in the 210 vessel segments (14\%) in the patients 
without IAT (IAT-group vs. non-IAT group, p-value 0.04). In the IAT-group, there were more foci of vessel wall enhancement ipsilateral $(n=18.5 ; 86 \%)$ to the treated artery compared with the contralateral side $(n=3 ; 14 \%)(p=0.005)$. In the nonIAT group there was no difference in the number of enhancing foci between the ipsilateral $(n=18 ; 60 \%)$ and the contralateral sides $(n=12 ; 40 \%)(p=0.47)$. In the IAT-group, vessel wall enhancement was more often concentric on the ipsilateral side $(n=8)$ compared with contralateral side $(n=0)(p=0.01)$. In the non-IAT group there was no significant difference in concentric enhancement between the ipsilateral $(n=7)$ and the contralateral side $(n=5)(p=0.14)$. No differences were found between eccentric enhancing foci ipsilateral-versus-contralateral side and IAT-group versus non-IAT group. The locations of all enhancing foci are shown in Table 2 and Supplemental table 1 and all statistical analyses are shown in Table 3. The degree of movement calculated over all patients was $0.77 \pm 0.51$ (standard deviation; SD) degrees for $\Delta$ Rotation and $0.81 \pm 0.59$ millimeters for $\Delta$ Translation.

Table 3. Comparison of enhancing foci between IAT-group and non-IAT group and between ipsilateral and contralateral side.

\begin{tabular}{|c|c|c|c|}
\hline & IAT-group & non-IAT group & p-value \\
\hline Total number of enhancing foci & 21.5 & 30 & $0.04 *$ \\
\hline Total number of ipsilateral enhancing foci & 18.5 & 18.0 & $0.003 *$ \\
\hline Total number of contralateral enhancing foci & 3.0 & 12.0 & $0.74 *$ \\
\hline Total number of concentric ipsilateral foci & 9.0 & 8.5 & $0.02 *$ \\
\hline \multirow[t]{2}{*}{ Total number of eccentric ipsilateral foci } & 9.5 & 9.5 & $0.07 *$ \\
\hline & (proportion); p-value & (proportion); p-value & \\
\hline $\begin{array}{l}\text { Total number of enhancing foci ipsilateral } \\
\text { vs. contralateral }\end{array}$ & (18.5 vs. 3.0$) ; \mathbf{0 . 0 0 5 ^ { \dagger }}$ & (18.0 vs. 12.0$) ; 0.47^{\dagger}$ & \\
\hline $\begin{array}{l}\text { Total number of concentric enhancing foci } \\
\text { ipsilateral vs. contralateral }\end{array}$ & $(9.0$ vs. 0.0$) ; \mathbf{0 . 0 1 1}^{\dagger}$ & (8.5 vs. 3.0$) ; 0.14^{+}$ & \\
\hline $\begin{array}{l}\text { Total number of eccentric enhancing foci } \\
\text { ipsilateral vs. contralateral }\end{array}$ & $(9.5$ vs 3.0$) ; 0.06^{+}$ & (9.5 vs. 9.0$) ; 0.97^{\dagger}$ & \\
\hline
\end{tabular}

Table 3. IAT: intra-arterial treatment; *, Mann-Whitney U test; $\mathrm{t}$, Wilcoxon Signed Rank test 

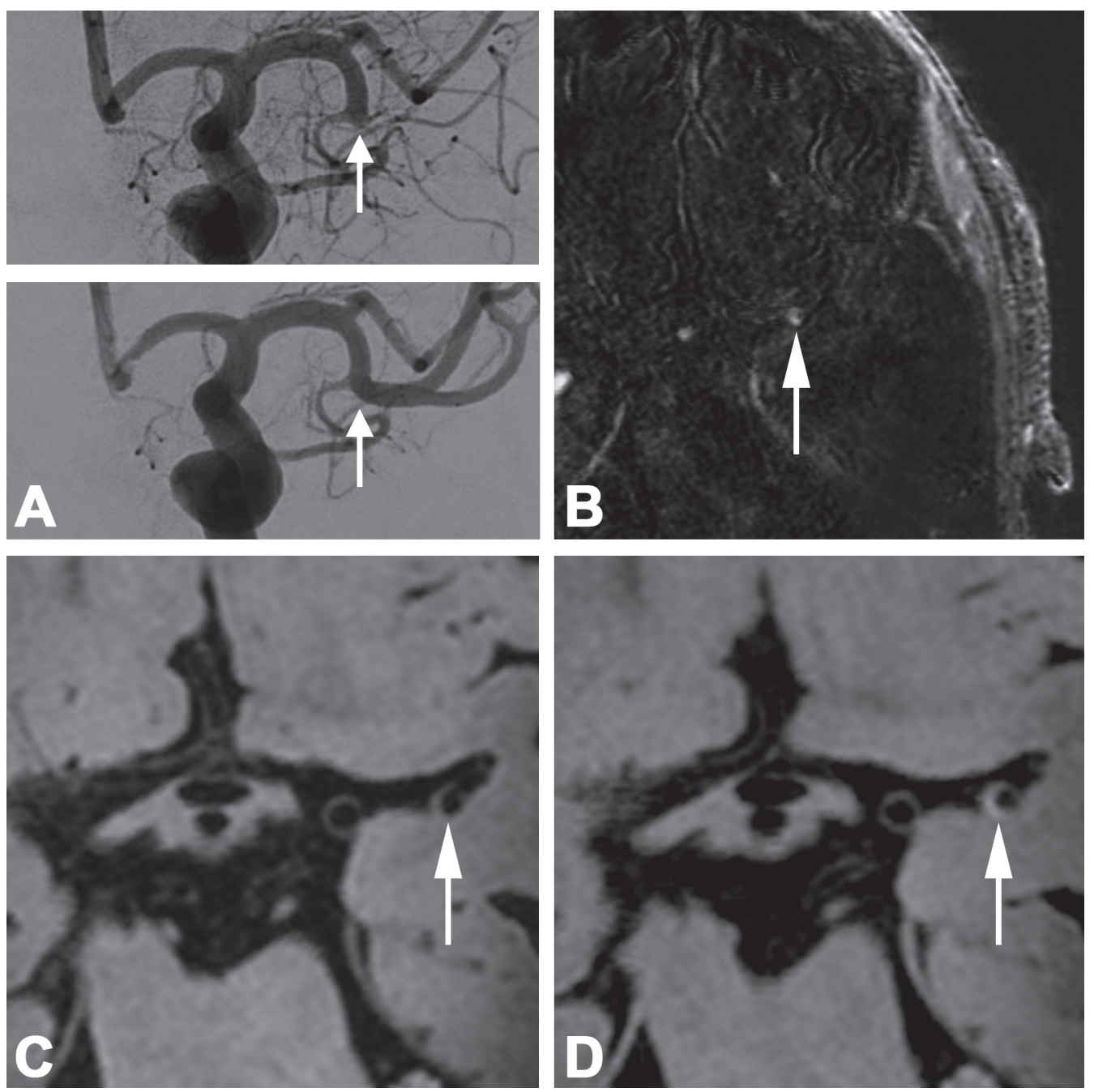

Figure 2. An 85-year-old male with an occlusion of the left M1-M2 segment (arrow), successfully treated with a thrombosuction device (patient number 9 in Table 2). (A) Digital subtraction angiography directly before and after thrombectomy procedure. (B) Subtraction image from coregistered pre- and postcontrast vessel wall images confirms a hyperintense configuration at the thrombectomy site. The transverse pre(C) and postcontrast (D) magnetization-prepared inversion recovery turbo spin-echo (MPIR-TSE) vessel wall images at 7T (voxel size $0.8 \times 0.8 \times 0.8 \mathrm{~mm}^{3}$ ) were obtained 22 days after thrombectomy procedure. The eccentric vessel wall enhancement present after contrast administration is at the same location as the thrombectomy site (arrow) (D). 

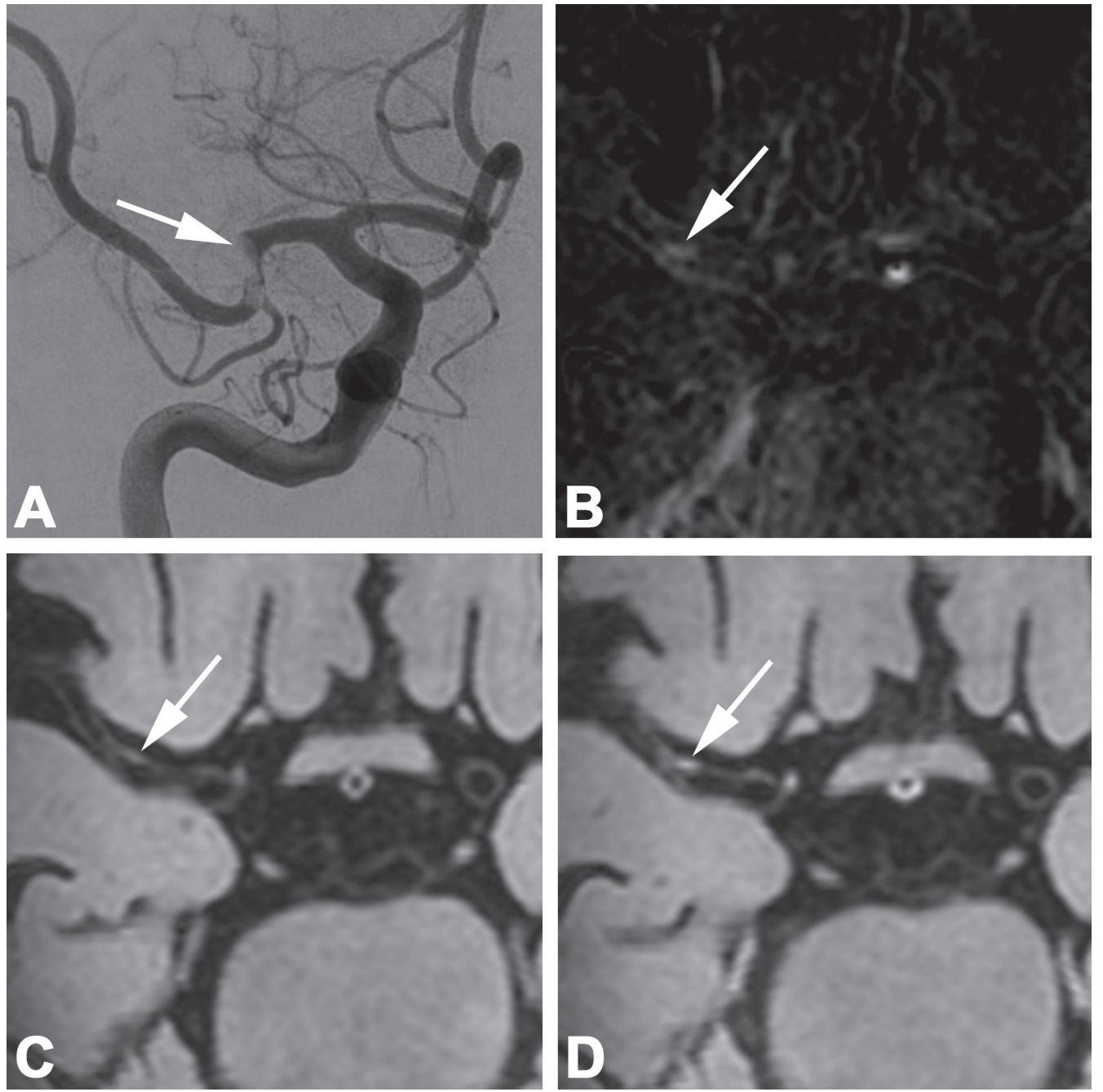

Figure 3. A 72-year-old female with an occlusion of the right M1 segment, successfully treated with a thrombosuction device (patient number 11 in Table 2). Digital subtraction angiography directly before thrombectomy procedure shows an acute occlusion in the right middle cerebral artery (A; arrow). (B) Subtraction image from coregistered pre- and postcontrast (C and $\mathbf{D}$ ) vessel wall images confirm a hyperintense rim (arrow) at the vessel wall. Transverse pre- (C) and postcontrast (D) magnetizationprepared inversion recovery turbo spin-echo (MPIR-TSE) vessel wall images at 7T were obtained 25 days after the thrombectomy procedure. The area of eccentric vessel wall enhancement (D) is seen in the right M1-segment, at the same location as the thrombectomy site, confirmed on the subtraction image in $\mathbf{B}$ (arrows). 

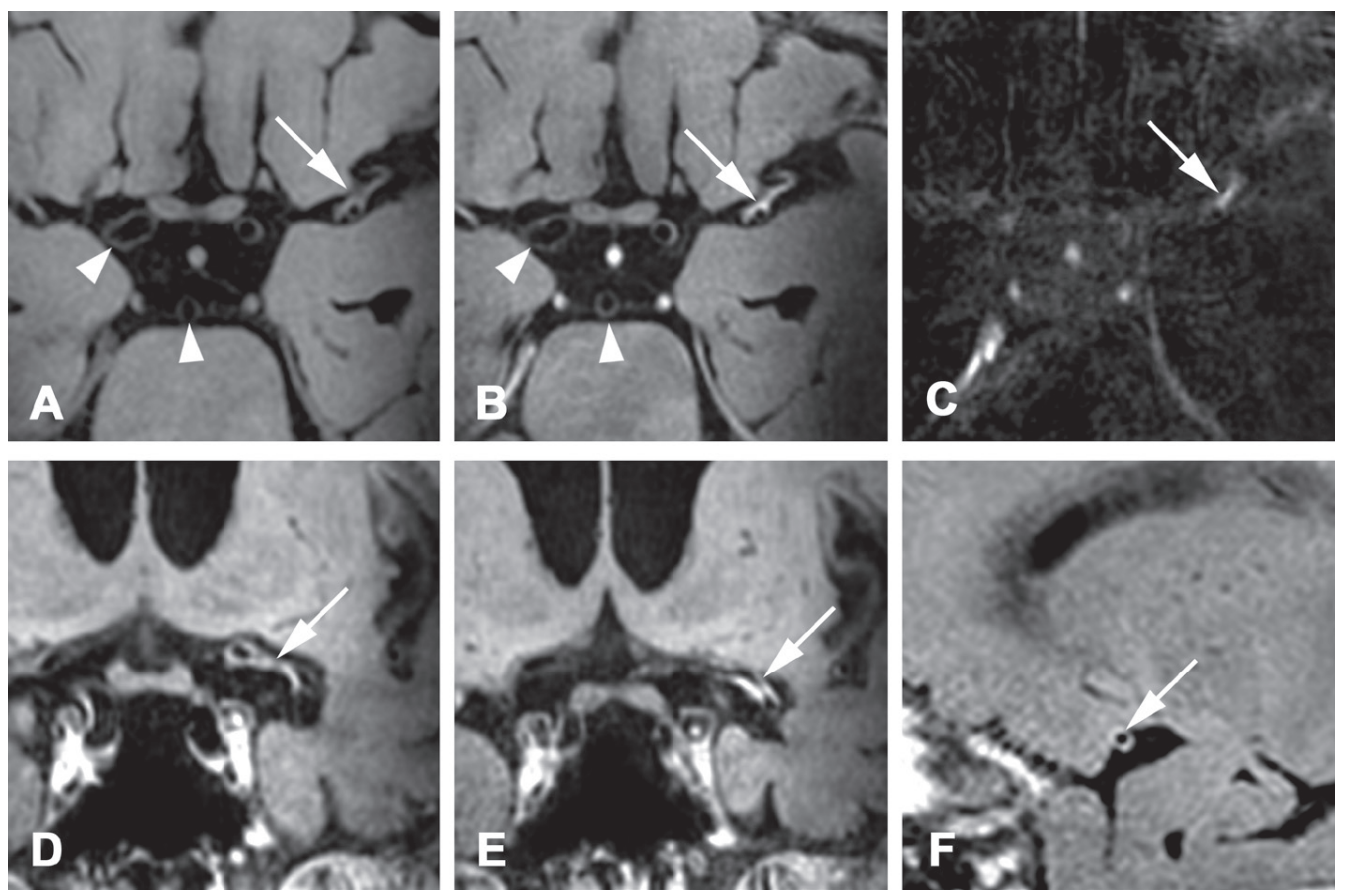

Figure 4. A 67-year-old male with an occlusion of the left M1-M2 segment, successfully treated with intraarterial thrombectomy (patient number 14 in Table 2). Patient was treated with both a stent-retriever device and a thrombosuction device. Axial pre- (A) and postcontrast (B) magnetization-prepared inversion recovery turbo spin-echo (MPIR-TSE) vessel wall images at 7T 87 days after the thrombectomy procedure. Clear contrast enhancement is present (white arrows), at the same location as the thrombectomy was performed. The carotid and basilar arteries appear normal (white arrowheads in $\mathbf{A}$ and $\mathbf{B}$ ). (C) Subtraction image of the pre- and postcontrast vessel wall images confirms the enhancement at the same location. (D and E) Coronal views of the postcontrast MPIR-TSE vessel wall images show enhancement over a long trajectory of the left M1 and M2 segments. (F) Sagittal view of the postcontrast MPIR-TSE vessel wall image shows that the enhancement has a concentric configuration. 

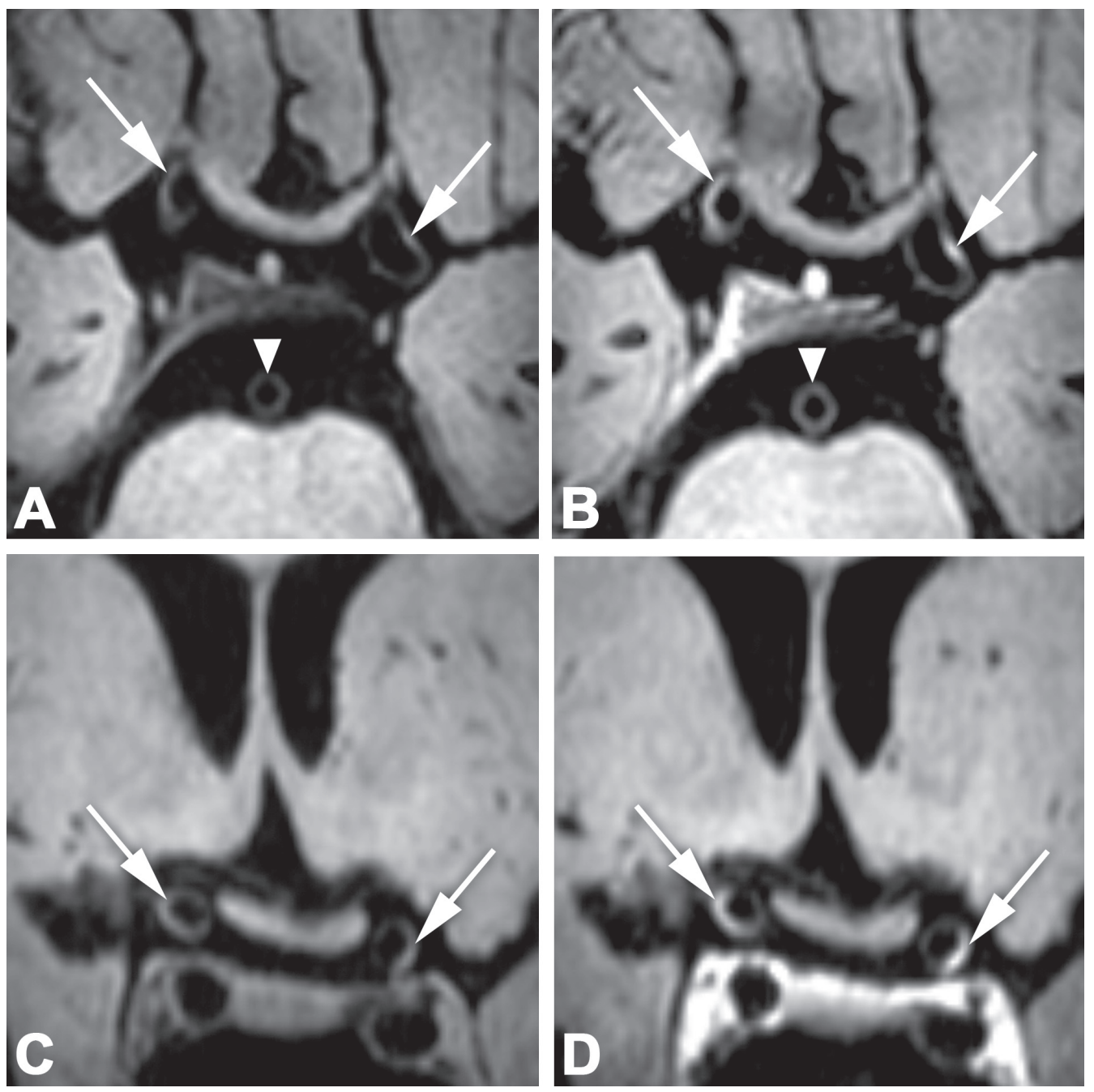

Figure 5. A 75-year-old female with an anterior circulation ischemic infarction of the left MCA territory (non-IAT group, number 30 in Supplemental table 1). Transverse and coronal precontrast (A and C) and postcontrast ( $\mathbf{B}$ and $\mathbf{D}$ ) magnetization-prepared inversion recovery turbo spin-echo (MPIR-TSE) vessel wall images at $7 \mathrm{~T}$ were performed 10 days after symptom onset of the ischemic infarction. Eccentric vessel wall enhancement is seen of the supra-clinoid portion of both intracranial carotid arteries ( $\mathbf{B}$ and D; arrows). The basilar artery appears normal (white arrowheads in $\mathbf{A}$ and $\mathbf{B}$ ). 


\section{Discussion}

In the current study, we show the patterns of vessel wall changes that can be anticipated to be seen in diagnostic vessel wall MRI studies in patients with stroke after IAT with a thrombosuction device compared with patients with stroke in whom no thrombectomy is performed. MR vessel wall imaging, in the subacute and chronic stages after IAT, showed more foci of vessel wall enhancement on the side treated with thrombectomy, while no difference between ipsi- and contralateral enhancement was found in the patients with stroke without IAT. Additionally, in the IAT-group, these enhancing foci were more often concentric in the vessels ipsilateral to the thrombectomy site compared with the contralateral side.

The higher number of ipsilateral foci of (concentric) contrast enhancement may be explained by several mechanisms: 1 ) the direct effect of the mechanical forces of the used thrombosuction device; 2 ) the indirect effect of the removed occluded thrombus by the release of (local) inflammatory molecules and 3) pre-existent atherosclerotic plaques with possible active inflammation. First, the direct effects of the mechanical forces of the thrombectomy procedure may induce vessel wall changes that cause vessel wall enhancement. This potential explanation is supported by previous imaging studies that studied the effect of IAT using a stentretriever (and one also including patients treated with a thrombosuction device) on the vessel wall on 3T MRI. ${ }^{10-13,24}$ The study that also included patients with a thrombosuction device showed that concentric vessel wall enhancement, which occurred in respectively $67 \%$ and $14 \%$ of the patients in the IAT-group ( $n=15$ ) versus the non-IAT group $(n=14)$, was associated with the number of attempts, type of device and more frequent hemorrhagic transformation of the ischemic infarct (mean interval treatment - MRI; $3.66 \pm 1.52$ days)..$^{13}$

In another series (IAT-group $n=6$ vs. non-IAT group $n=10$ ), MR vessel wall imaging showed wall thickening in $83 \%$ versus $30 \%$ and contrast enhancement in $67 \%$ versus $20 \%$ of patients, respectively, (mean interval treatment - MRI; $3.44 \pm 2.58$ days). ${ }^{11}$ Also, in a recent study more contrast-enhancing vessel wall areas were found in the post-thrombectomy group $(n=6)$, scanned within 24 hours after treatment, compared with a control group $(n=5) .{ }^{12}$ In addition, some follow-up studies (ranging from several days up to two years) reported delayed arterial vessel wall abnormalities, postulating that these might be attributable to endothelial damage after IAT.3,6,8,25,26 On the other hand, in a study with 23 stroke patients who were scanned within one week after thrombectomy, major vessel wall damage such as disruption and stenosis were rare. ${ }^{10}$

We limited our study to patients treated with a thrombosuction device. In this study $7 \mathrm{~T}$ MRI was used, but previous 3T MRI and 7T MRI comparison studies 
showed that 3T MRI vessel wall imaging can also detect most vessel wall lesions. 17,18 Furthermore, previous 3T MRI studies assessing vessel wall enhancement after treatment with stent-retriever devices have comparable findings. ${ }^{10-13,24}$ Second, the observed vessel wall enhancement may be a result of the former occluding thrombus on the arterial vessel wall that causes reactive, inflammatory changes or a local 'scarring' of the vessel wall. This is supported by studies that showed an increased level of inflammatory markers after an ischemic stroke. ${ }^{27,28} \mathrm{~A}$ third, more hypothetical explanation for the higher number of ipsilateral foci of contrast enhancement is the presence of pre-existent intracranial atherosclerotic lesions, possibly with active inflammation, in the revascularized segment. ${ }^{29}$ However, the absence of a significant difference in enhancement between the ipsi- and contralateral arteries in our non-IAT group suggests that not all of the observed enhancing foci can be explained by pre-existent atherosclerotic lesions. Furthermore, atherosclerotic lesions often have a more eccentric configuration, which is in contrast to the high number of concentric type of enhancing foci seen in our study and might indicate an inflammatory state of the whole vessel wall rather than an eccentric atherosclerotic plaque..$^{22}$

It is unknown how long contrast enhancement of the arterial vessel wall persists after IAT in patients with stroke. In our study we used a relatively long time interval between thrombectomy and MR imaging (up to three months) compared with previously published papers (range 1-11 days). ${ }^{10,11}$ Our results indicate that vessel wall enhancement persists also in the subacute-to-chronic phase.

This study has limitations. First, because local areas of ischemia appear as hypointense parenchymal lesions on the $\mathrm{T}_{1}$-weighted vessel wall sequences, we were unable to blind the observers to the side of thrombectomy in the IATgroup or side of infarction in the non-IAT group (left or right). Second, IAT was introduced in our center as standard clinical care during the study inclusion period, after the successful international IAT trials. Therefore, the first 28 patients in the non-IAT group did not have IAT as a treatment option. Retrospectively, 10 of all 35 patients without IAT had a proximal occlusion (ICA, M1 or M2) similar to the IAT patients. In the remaining 25 patients without IAT no acute occlusion and a better overall NIHSS score was recorded in the final reports (Supplemental table 1). This result may have led to a selection bias in the non-IAT group with the inclusion of lesser affected patients that may have biased the true results. Nevertheless, we feel that the comparison of the ipsilateral to contralateral side in the IAT-group is most relevant because in this comparison, all potential individual risk factors are the similar. Third, the number of IAT patients enrolled in this study is relatively low. Including more patients would benefit the statistical power and enable the possibility of additional analyses. Fourth, for most patients without IAT, the time window between symptom onset and 7T MRI was shorter. 
Nevertheless, there were significant more enhancing foci in the IAT-group, despite the longer time window. Finally, discrimination between eccentric or concentric enhancing foci can be challenging and in clinical practice, certainly there is an overlap in enhancement configuration and the causes of vessel wall enhancement. Histopathologic validation of the detected enhancement would have given insight into the true nature / composition of the enhancing vessel wall areas (due to IAT procedure, thrombus-related or pre-existing atherosclerotic plaques); however, this necessitates ex vivo tissue which was not available. Therefore, some of the enhancing foci detected in our study participants might be explained by one of these other mechanisms of wall enhancement. Patients should ideally also have been scanned before the IAT procedure, but due to the limited time frame in which IAT can be performed, this is difficult to accomplish in clinical practice.

\section{Conclusions}

IAT using thrombosuction did show more (concentric) enhancing foci of the vessel wall ipsilateral to the occlusion site compared with the patients without IAT, suggesting reactive changes of the vessel wall. In an era in which MRI vessel wall studies are expected to be increasingly performed in the diagnostic workup and follow-up of patients with acute stroke, the patterns of vessel walls enhancement after thrombectomy need to be known to avoid misinterpretation of these enhancing patterns in the follow-up MRI examinations after acute stroke treatment. 


\section{References}

1. Berkhemer OA, Fransen PS, Beumer D, et al. A randomized trial of intraarterial treatment for acute ischemic stroke. The New England Journal of Medicine 2015;372:11-20.

2. Rodrigues FB, Neves JB, Caldeira D, Ferro JM, Ferreira JJ, Costa J. Endovascular treatment versus medical care alone for ischaemic stroke: systematic review and meta-analysis. BMJ 2016;353:i1754.

3. Gory B, Bresson D, Kessler I, et al. Histopathologic evaluation of arterial wall response to 5 neurovascular mechanical thrombectomy devices in a swine model. AJNR American Journal of Neuroradiology 2013;34:2192-8.

4. Teng D, Pannell JS, Rennert RC, et al. Endothelial trauma from mechanical thrombectomy in acute stroke: in vitro live-cell platform with animal validation. Stroke 2015;46:1099-106.

5. Peschillo S, Diana F, Berge J, Missori P. A comparison of acute vascular damage caused by ADAPT versus a stent retriever device after thrombectomy in acute ischemic stroke: a histological and ultrastructural study in an animal model. Journal of Neurointerventional Surgery 2017;9:743-749.

6. Kurre W, Perez MA, Horvath D, et al. Does mechanical thrombectomy in acute embolic stroke have long-term side effects on intracranial vessels? An angiographic follow-up study. Cardiovascular and Interventional Radiology 2013;36:629-36.

7. The penumbra pivotal stroke trial: safety and effectiveness of a new generation of mechanical devices for clot removal in intracranial large vessel occlusive disease. Stroke 2009;40:2761-8.

8. Renu A, Laredo C, Lopez-Rueda A, et al. Vessel Wall Enhancement and BloodCerebrospinal Fluid Barrier Disruption After Mechanical Thrombectomy in Acute Ischemic Stroke. Stroke 2017;48:651-657.

9. Gascou G, Lobotesis K, Machi P, et al. Stent retrievers in acute ischemic stroke: complications and failures during the perioperative period. AJNR American Journal of Neuroradiology 2014;35:734-40.

10. Hsieh K, Verma RK, Schroth G, et al. Multimodal 3 Tesla MRI confirms intact arterial wall in acute stroke patients after stent-retriever thrombectomy. Stroke 2014;45:3430-2.

11. Power S, Matouk C, Casaubon LK, Silver FL, Krings T, Mikulis DJ. Vessel wall magnetic resonance imaging in acute ischemic stroke: effects of embolism and mechanical thrombectomy on the arterial wall. Stroke 2014;45:2330-4.

12. Abraham P, Scott Pannell J, Santiago-Dieppa DR, et al. Vessel wall signal enhancement on 3-T MRI in acute stroke patients after stent retriever thrombectomy. Neurosurgical Focus 2017;42:E20.

13. Seo WK, Oh K, Suh SI, Seol HY. Clinical Significance of Wall Changes After Recanalization Therapy in Acute Stroke: High-Resolution Vessel Wall Imaging. Stroke 2017;48:1077-1080.

14. Van der Kolk AG, Zwanenburg JJ, Brundel M, et al. Intracranial vessel wall imaging at 7.0T MRI. Stroke 2011;42:2478-84. 
15. Van der Kolk AG, Zwanenburg JJ, Brundel M, et al. Distribution and natural course of intracranial vessel wall lesions in patients with ischemic stroke or TIA at 7.0 Tesla MRI. European Radiology 2015;25:1692-700.

16. Van der Kolk AG, Hendrikse J, Brundel M, Biessels GJ, Smit EJ, Visser F. Multi-sequence whole-brain intracranial vessel wall imaging at 7.0 tesla. European Radiology 2013;23:2996-3004.

17. Harteveld AA, van der Kolk AG, van der Worp HB, et al. High-resolution intracranial vessel wall MRI in an elderly asymptomatic population: comparison of 3T and 7T. European Radiology 2017;27:1585-1595.

18. Zhu $C$, Haraldsson $H$, Tian $B$, et al. High resolution imaging of the intracranial vessel wall at 3 and 7 T using 3D fast spin echo MRI. MAGMA 2016;29:559-70..

19. Fugate JE, Klunder AM, Kallmes DF. What is meant by "TICI"? AJNR American Journal of Neuroradiology 2013;34:1792-7.

20. Samuels OB, Joseph GJ, Lynn MJ, Smith HA, Chimowitz MI. A standardized method for measuring intracranial arterial stenosis. AJNR American Journal of Neuroradiology 2000;21:643-6.

21. Lindenholz A, van der Kolk AG, Zwanenburg JJM, Hendrikse J. The Use and Pitfalls of Intracranial Vessel Wall Imaging: How We Do It. Radiology 2018;286:12-28.

22. Swartz RH, Bhuta SS, Farb RI, et al. Intracranial arterial wall imaging using high-resolution 3-tesla contrast-enhanced MRI. Neurology 2009;72:627-34.

23. Klein S, Staring M, Murphy K, Viergever MA, Pluim JP. elastix: a toolbox for intensity-based medical image registration. IEEE Transactions on Medical Imaging 2010;29:196-205.

24. Li Y, Turan TN, Chaudry I, et al. High-Resolution Magnetic Resonance Imaging Evidence for Intracranial Vessel Wall Inflammation Following Endovascular Thrombectomy. Journal of Stroke and Cerebrovascular Diseases 2017;26:e96-e98.

25. Akpinar S, Yilmaz G. Early middle cerebral artery stenosis following stent-assisted thrombectomy. Interventional Neuroradiology 2015;21:337-40.

26. Eugene F, Gauvrit JY, Ferre JC, et al. One-year MR angiographic and clinical followup after intracranial mechanical thrombectomy using a stent retriever device. AJNR American Journal of Neuroradiology 2015;36:126-32.

27. Schuhmann MK, Gunreben I, Kleinschnitz C, Kraft P. Immunohistochemical Analysis of Cerebral Thrombi Retrieved by Mechanical Thrombectomy from Patients with Acute Ischemic Stroke. International Journal of Molecular Sciences 2016;17:298.

28. Jin R, Liu L, Zhang S, Nanda A, Li G. Role of inflammation and its mediators in acute ischemic stroke. Journal of Cardiovascular Translational Research 2013;6:834-51.

29. Qiao Y, Zeiler SR, Mirbagheri S, Leigh R, Urrutia V, Wityk R. Intracranial plaque enhancement events on in patients with cerebrovascular high-spatial-resolution MR images. Radiology 2014;271.

30. Chung JW, Park SH, Kim N, et al. Trial of ORG 10172 in Acute Stroke Treatment (TOAST) classification and vascular territory of ischemic stroke lesions diagnosed by diffusionweighted imaging. Journal of the American Heart Association 2014;3:e001119. 


\section{Supplemental Material}

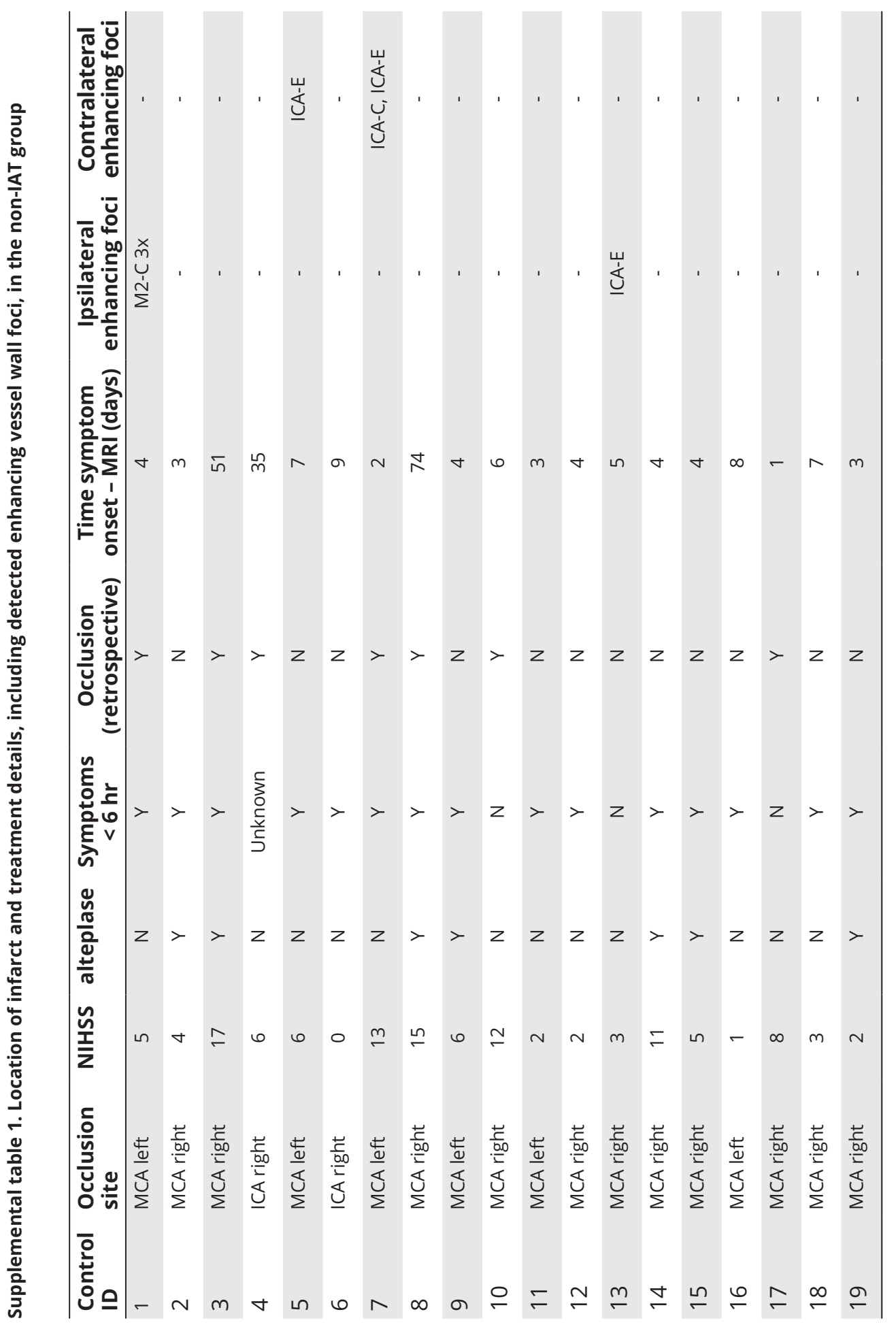




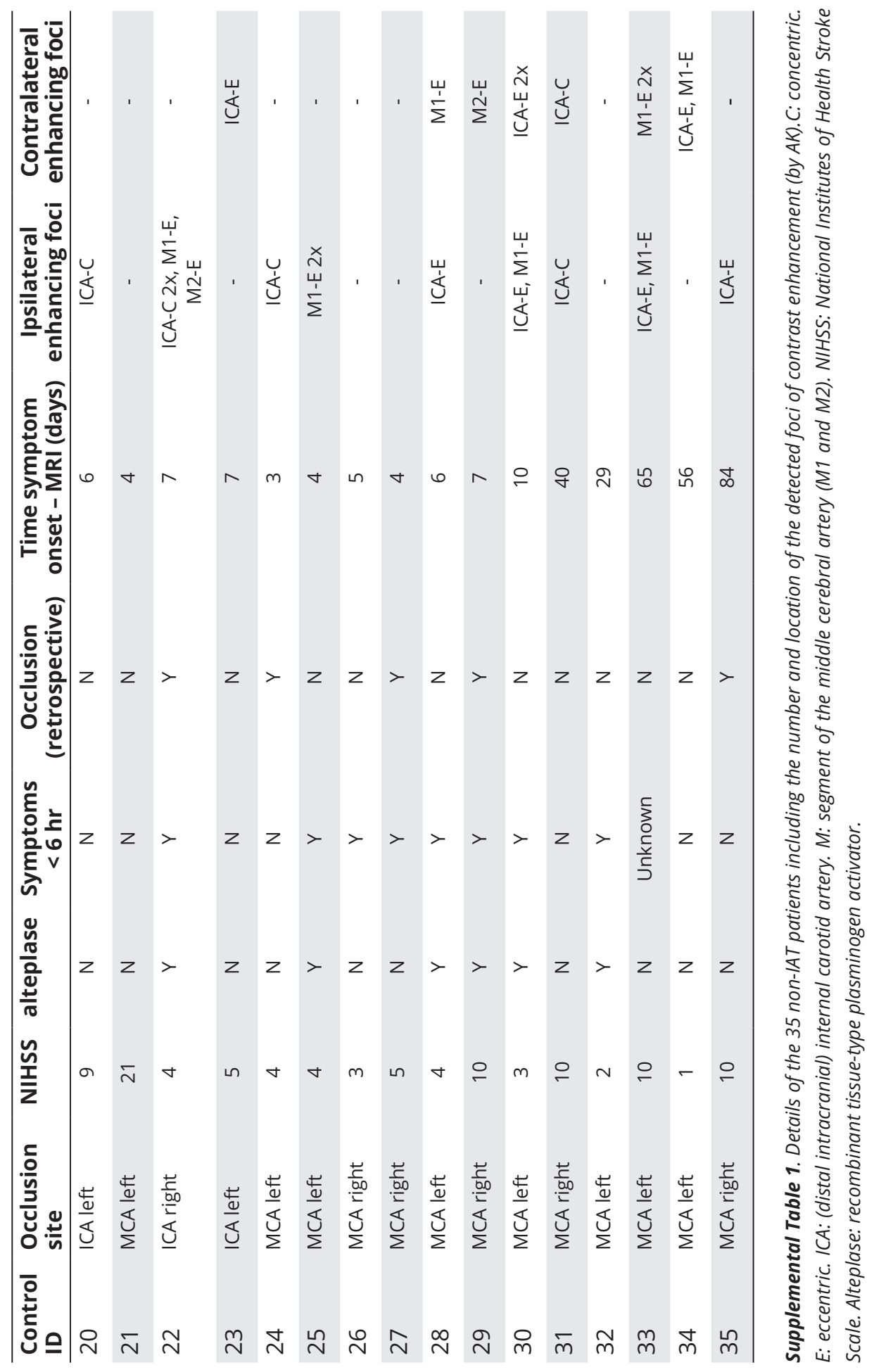


MRI Vessel Wall Imaging after Intra-Arterial Treatment for Acute Ischemic Stroke 
Summary and General Discussion 


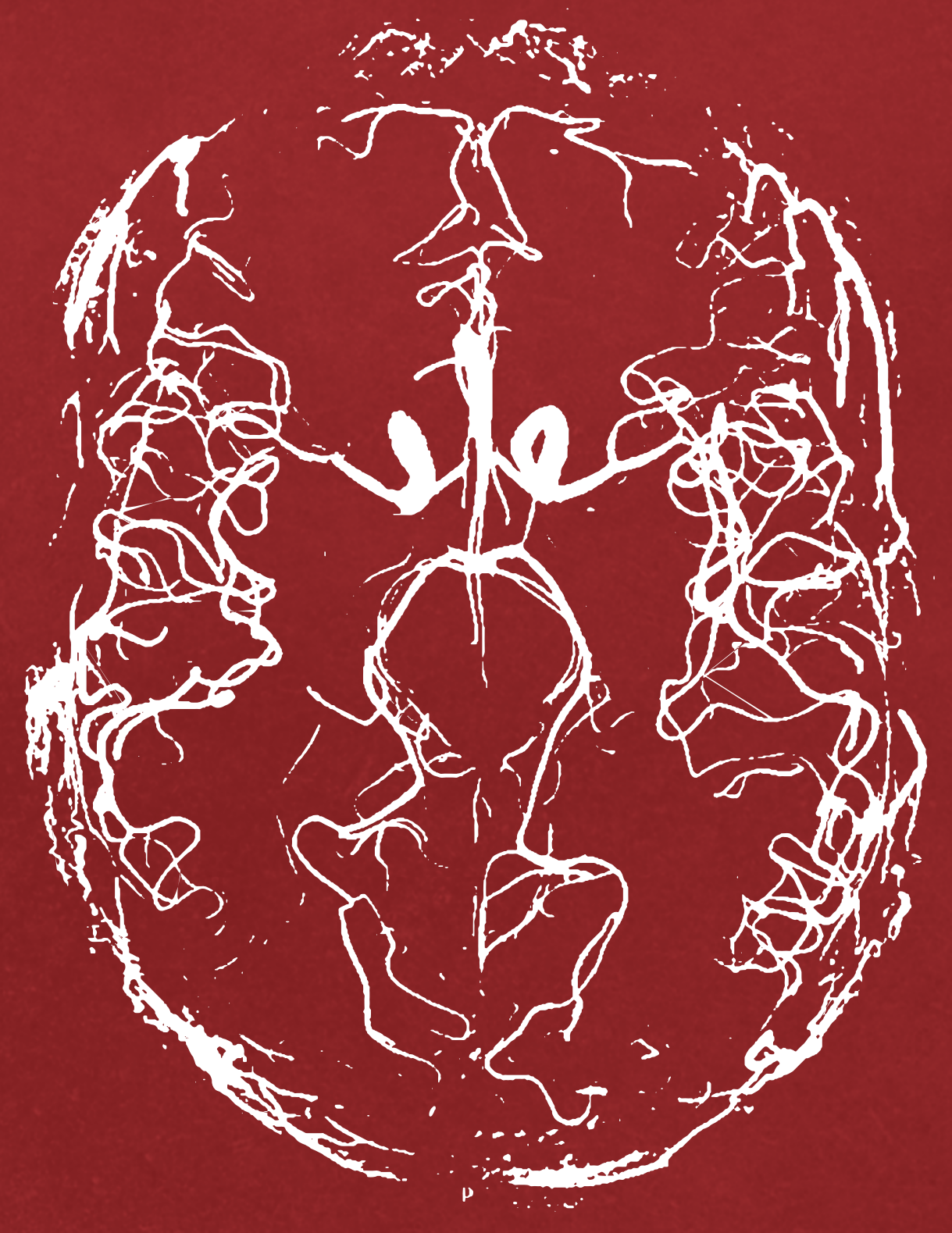




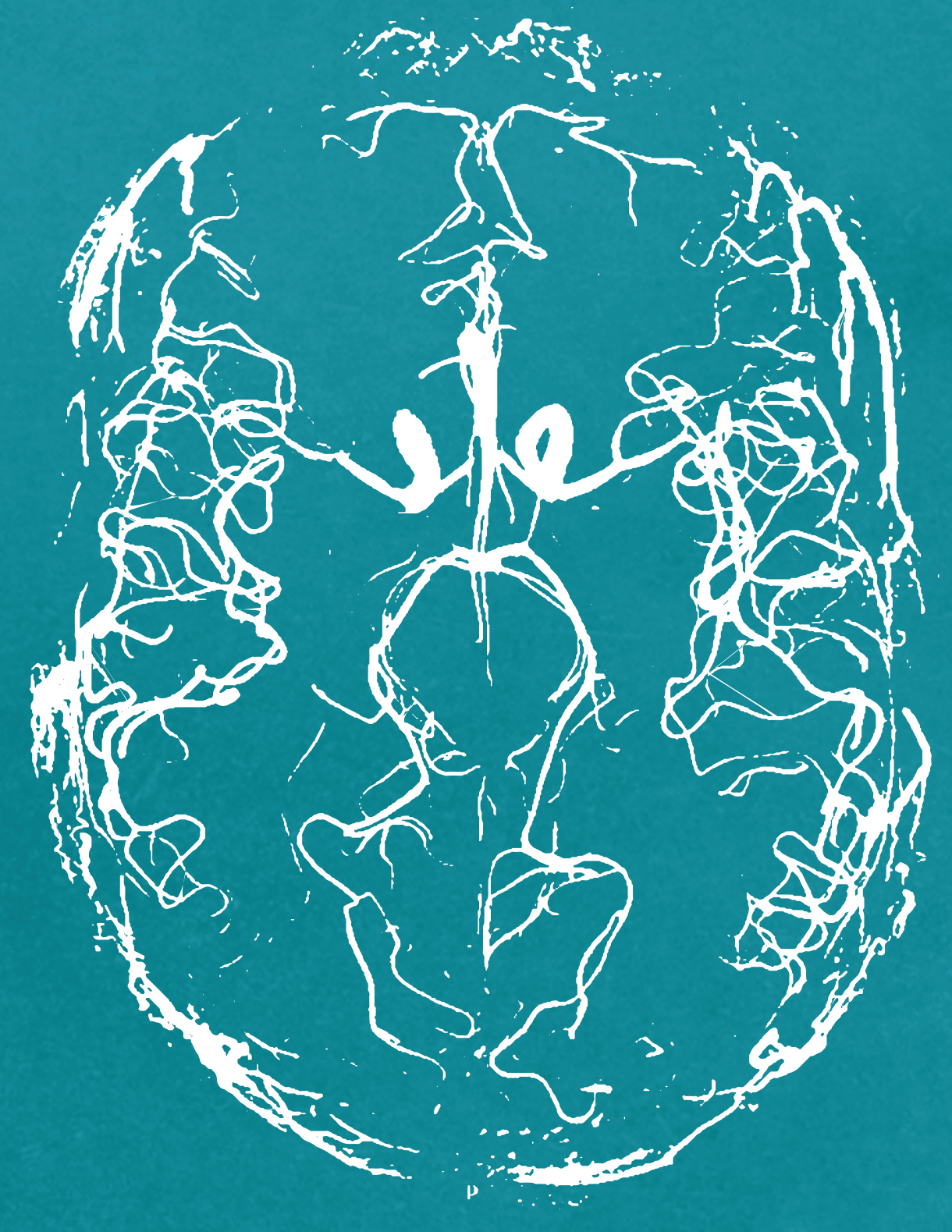


CHAPTER

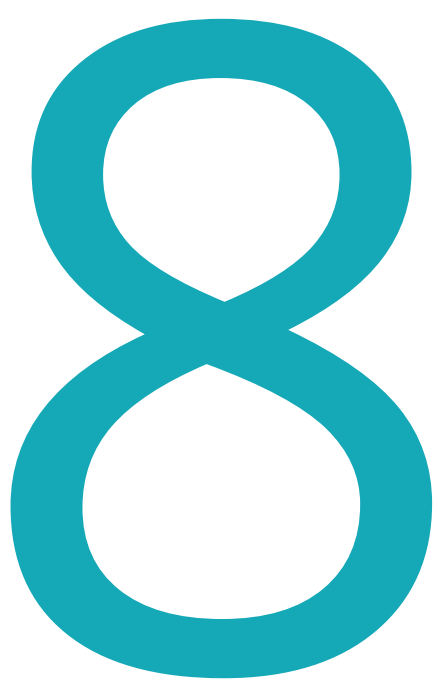

Summary 
Over the last two decades vessel wall MRI has seen many technical improvements and an associated increase in clinical applicability. While initially only the large extracranial arteries could be visualized, nowadays several sequences are available for imaging the intracranial arteries as well, facilitated by technical advances as well as higher achievable signal-to-noise ratio (SNR), contrast-to-noise ratio (CNR) and spatial resolution at increasing magnetic field strengths ( $3 \mathrm{~T}$ and $7 \mathrm{~T}$ ). As a result, the submillimeter thin intracranial vessel wall can now be visualized and scrutinized for abnormalities, and new imaging features for a variety of vascular pathologies have become available. Nevertheless, intracranial vessel wall MRI has not been systematically applied in the clinical setting yet, mainly because of the difficulty in obtaining histopathological validation of the true nature of the detected MR findings and the lack of studies addressing the clinical value of intracranial vessel wall lesions in patient management.

The first part of this thesis addressed the technical aspects and challenges of intracranial vessel wall MRI in relation to magnetic field strength and acquisition time. An overview of the advantages and challenges of using 7T MRI in vascular neurological diseases including ischemic stroke was given in Chapter 2. The differences, similarities and general challenges of specifically intracranial vessel wall MRI at 3T and 7T were discussed in Chapter 3. Whereas 3T as imaging platform of the intracranial vessel wall is more practical from a clinical standpoint, can achieve high-resolution images of the vessel wall, and can aid in differentiating between vascular pathologies, 7T MRI has the advantage of clearer delineation of the vessel wall due to superior CFS suppression, and generally visualizes more lesions. In Chapter $\mathbf{3}$ we also attempted to aid the radiologist in assessing intracranial vessel wall MR images, i.e., what to look for (and what to look out for), how to interpret abnormalities and which pathology to diagnose. Chapter $\mathbf{4}$ addressed the long acquisition times of most intracranial vessel wall MRI sequences, caused by the time-demanding sequence parameters. By adapting spatial resolution, SNR, CNR or a combination of these factors, various intracranial vessel wall sequences were tested for image quality. The results demonstrated that acquisition time could be reduced by $30 \%$ compared with the current clinically used sequence while maintaining both qualitative and quantitative satisfactory image quality.

The second part of this thesis attempted to further elucidate the nature of vessel wall lesions (in an indirect way) and, consequently, the role intracranial vessel wall MRI could play in clinical decision-making. In Chapter 5, we showed that in a group of patients with ischemic stroke or TIA, several cardiovascular risk factors including increasing age, diabetes mellitus and hypertension - were associated with a higher number of intracranial vessel wall lesions. In the same population, Chapter 6 demonstrated that a higher number of vessel wall lesions was associated with the presence of cerebral parenchymal damage, including presence of infarcts often 
caused by small vessel disease and moderate to severe periventricular white matter hyperintensities. The results of these studies (I) tentatively support the notion that the detected vessel wall lesions most likely represent true atherosclerotic lesions rather than for example normal variations in vessel wall thickness; (II) suggest a risk factor profile for the development of intracranial vessel wall lesions comparable with that for extracranial atherosclerosis, which might be addressed with risk factor reduction; and (III) show the relation of these vessel wall lesions with damage to the cerebral parenchyma. Nonetheless, larger studies will be necessary to assess whether medical intervention (e.g., treatment with antihypertensive drugs) indeed changes lesion burden and/or cerebral damage. Finally, in Chapter $\mathbf{7}$ intracranial vessel wall MRI was used as non-invasive imaging technique to assess possible vessel wall damage after intra-arterial thrombectomy (IAT). Vessel wall enhancement was seen more frequently and in a concentric configuration on the ipsilateral side in patients treated by IAT than in patients who did not receive this treatment, suggesting reactive changes of the vessel wall after IAT which should be taken into consideration during follow-up MRI of the vessel wall.

This thesis shows the potential value of intracranial vessel wall MRI in clinical practice; next steps in advancing clinical translation of intracranial vessel wall MRI are histopathological validation of detected vessel wall lesions, setup of larger studies to identify the exact association between vessel wall lesions, clinical characteristics and clinical outcome, and formation of international collaborative projects to standardize acquisition and assessment of vessel wall MR images. Ultimately, intracranial vessel wall MRI has the strong potential to be implemented in the workup and follow-up of patients with cerebrovascular disease, in risk management, and in diagnosing other vasculopathies, using it as non-invasive diagnostic tool to guide preventive and therapeutic decision-making. 


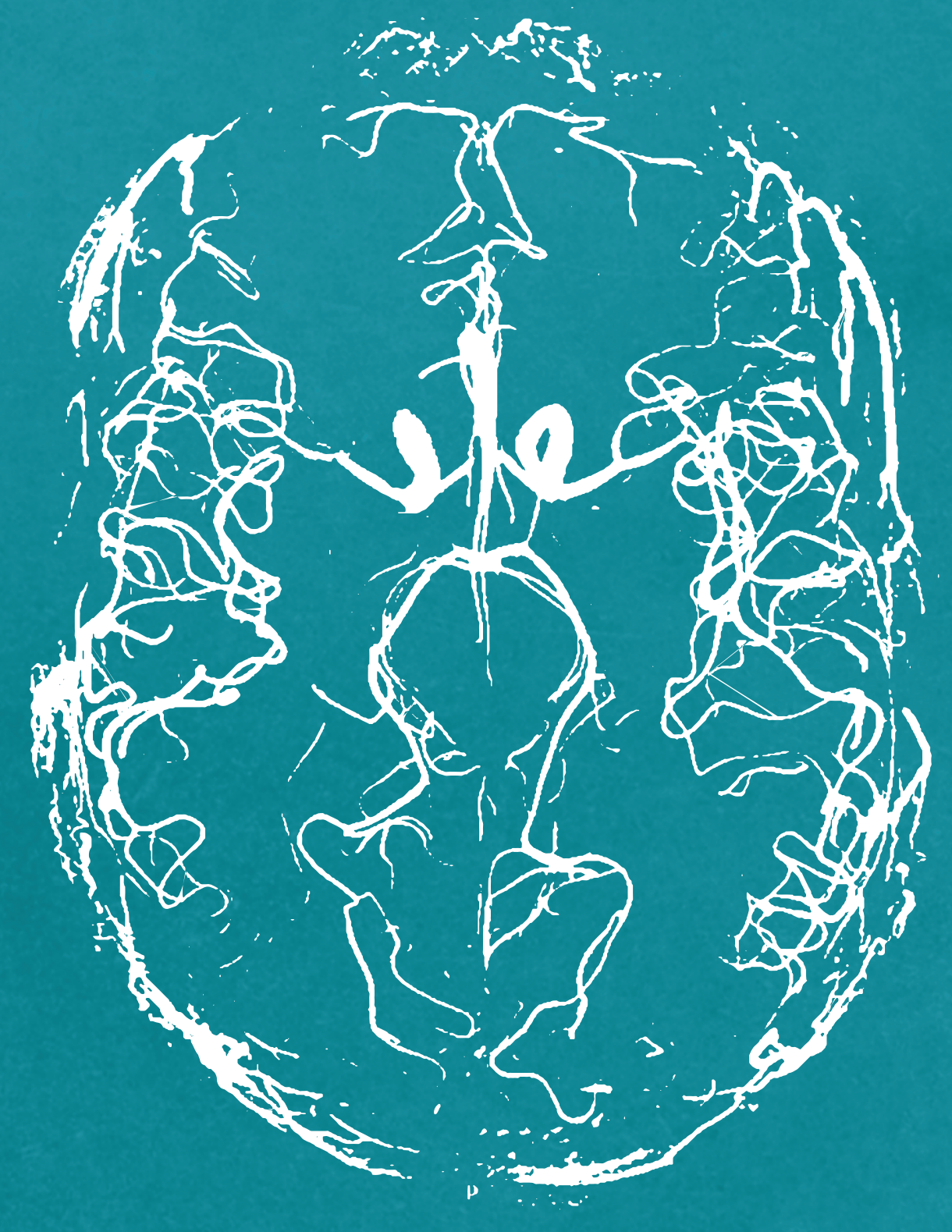


CHAPTER

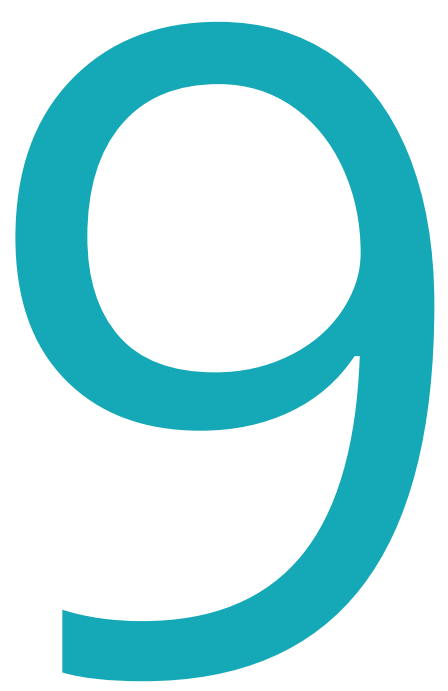

General Discussion 
Over the last two decades, intracranial vessel wall MRI has seen a steady increase in popularity, both in research and clinical practice. While before 2010 very few articles addressing intracranial vessel wall MRI were published annually, since that time publications have increased exponentially, with more than 80 published articles in 2019. ${ }^{1}$ Initially, efforts were set on technical developments to actually visualize the intracranial vessel wall with sufficient image quality, but when this was achieved a shift soon occurred towards potential clinical applications of intracranial vessel wall MRI, for instance in central nervous vasculitis (CNS) vasculitis and intracranial aneurysms. ${ }^{2-8}$ The real value of intracranial vessel wall MRI in clinical practice, however, has still not been entirely elucidated, thereby limiting its routine application in the clinic, mainly because of a lack of validation studies and difficulties in translating imaging findings to pathology. The goal of this thesis was to explore the current state of technical developments and clinical indications of intracranial vessel wall MR imaging, and to make the first steps from research-based knowledge towards interpretation of the value of vessel wall imaging findings in the clinical setting.

\section{Optimizing vessel wall sequences for clinical use}

Intracranial vessel wall MRI sequences suffer from a relative long acquisition time: most clinically applicable $3 T$ sequences have acquisition times varying between 5 to 10 min. ${ }^{9}$ When both pre- and postcontrast vessel wall imaging is required, acquisition time is doubled which makes it even more difficult to implement vessel wall sequences in existing clinical imaging protocols with predetermined time slot lengths. Further, motion artefacts will deteriorate image quality when acquisition times are too long, especially in neurologically compromised patients for whom it may be more difficult to lie still. However, as image quality is a balance between acquisition time, spatial resolution, signal-to-noise ratio (SNR) and contrast-tonoise ratio (CNR), time reduction is in conflict with the highly-demanding technical requirements for vessel wall MRI. For clinical applicability, it is important that the trade-offs in sequence parameters (and, for that matter, acquisition of pre- and/ or postcontrast images) are chosen in such way that the clinical question can be answered. This implies that clinical vessel wall MR sequences may be patienttailored or disease-tailored. In Chapter $\mathbf{4}$ a comparison was made between several $3 \mathrm{~T}$ vessel wall MRI sequences, showing that acquisition time could be reduced by $30 \%$ compared with existing clinical sequences. Although the overall technical image quality of the most optimized sequence was lower, qualitative image assessment was still acceptable, which makes this vessel wall MRI sequence more clinically applicable to implement in existing protocols. Ongoing technical developments may allow for higher spatial resolution without significant acquisition time extension in the future. Hardware developments like more advanced receiver coils with a larger field-of-view and a higher number of receiver 
channels, or advanced pulse sequence designs, such as compressed sensing, different blood and cerebrospinal fluid suppression techniques or more efficient $k$-space sampling trajectories may further optimize intracranial vessel wall MRI for use in clinical MRI protocols. ${ }^{4,8,10-14}$

\section{Validation of intracranial vessel wall findings}

When assessing intracranial vessel wall MR images, the vessel wall is basically being appreciated on two characteristics: thickening and enhancement. Vessel wall thickening is further specified by configuration (eccentric or concentric) and whether it has led to stenosis. Enhancement is similarly specified by configuration, and whether it follows a specific pattern (e.g., entire vessel wall, interleaving normal vessel wall segments or partly enhancing vessel wall thickening). ${ }^{4}$ For clinical implementation, it is essential that these vessel wall changes correlate with true abnormalities; histopathological validation of the detected vessel wall lesions is therefore crucial.

Contrary to most extracranial arteries, tissue samples from intracranial arteries (aside from surgically removed aneurysms and sporadic vessel biopsies) cannot be obtained while the patient is alive. ${ }^{15-18}$ The most ideal alternative is histopathological validation using tissue from deceased patients who consented to an autopsy procedure and underwent in vivo intracranial vessel wall MRI while still alive. So far, only a few studies using this setup have been published; with the increasing use of intracranial vessel wall MRI, this number may increase in the near future. ${ }^{19-21}$ A less ideal but an easier to perform alternative uses solely ex vivo MRI and histopathological examination, variably performed at 1.5T, 3T and 7T MRI. ${ }^{11-26}$ Studies using this approach showed a clear correlation between intracranial atherosclerotic plaques at histopathology, and the presence of vessel wall lesions on MR images. Its advantages are a higher achievable spatial resolution and lack of pulsatility or motion artefacts; however, fixation effects (such as cell shrinkage) and tissue temperature can change the MRI characteristics of the tissue. In addition, functional measures of the vessel wall like enhancement cannot be performed in postmortem tissue. This makes histopathological validation of certain vessel wall pathologies, such as inflammatory vasculopathies that show predominantly vessel wall enhancement in typical patterns, more difficult. Furthermore, other vasculopathies like intracranial aneurysms, moyamoya disease and post intra-arterial thrombectomy changes like those reported in Chapter $\mathbf{7}$ have not been histopathologically validated in this way yet. A third validation option is to extrapolate findings from extracranial vessel wall MRI, for which histopathological validation is much easier, for example because of the availability of carotid endarterectomy specimens..$^{21,27}$ This however needs to be done with caution because the intracranial vessel walls are anatomically and histologically different from extracranial arteries. ${ }^{28}$ 


\section{Interpreting vessel wall thickening}

Another challenge in vessel wall assessment is the used cutoff value for vessel wall thickening. Previous studies have shown that healthy participants also show vessel wall thickening, and that vessel wall diameter increases somewhat with age. ${ }^{29-}$

${ }^{31}$ It is currently not clear whether or up to which stage vessel wall thickening still represents normal age-related variation of the intracranial vessel wall, and what can be seen as early indicators of (non-stenotic) atherosclerotic plaques. In general, the vessel wall is considered radiologically thickened when thickness has increased with more than 50\% compared with the adjacent vessel wall (Chapter 3). ${ }^{4,32}$ However, this is an arbitrary cutoff value chosen for practical purposes and not validated. Most vessel wall pathologies that eventually result in wall thickening, especially intracranial atherosclerosis, have a continuous spectrum of vessel wall thickening that varies depending on disease severity; therefore, this cutoff point should be validated with histopathology and with clinical outcome. An additional question that arises in this regard is how much the vessel wall should be thickened to be reliably seen on MRI. Histopathological studies have shown that intracranial vessel walls of the circle of Willis range in thickness from $0.2-0.4 \mathrm{~mm}$; in contrast, radiological measurements have reported vessel wall thicknesses of approximately $1.0 \mathrm{~mm}$ for the large intracranial arteries of the circle of Willis. ${ }^{33,34}$ This difference may be caused by an underestimation in histopathological studies due to e.g., cell shrinkage, or an overestimation in radiological studies due to partial volume effects, motion artefacts and pulsatility effects, or both., ${ }^{4,35}$ The latter is supported by a recent study which showed that with currently used spatial resolutions for vessel wall MRI - with varying voxel sizes between $0.4-0.8 \mathrm{~mm}$ - vessel wall thicknesses cannot be reliably measured. ${ }^{35}$ To perform accurate vessel wall thickness measurements, one needs at least two voxels to prevent partial volume effects, necessitating voxel sizes of at least $0.18 \mathrm{~mm}$ isotropic (or smaller) which are currently not feasible within clinically reasonable acquisition times, not even at 7T.4,36 Notwithstanding these drawbacks, the fact that thickness variations of the vessel wall are visible using in vivo vessel wall sequences means that qualitative assessment of the intracranial vessel wall is possible, by inferring signal intensity and vessel wall thickness throughout the whole vascular tree and comparing these with the adjacent parts of the artery or with the contralateral arteries.

\section{Indirect evidence to the nature of vessel wall lesions}

Since the current relative lack of histopathological validation of intracranial vessel wall lesions precludes strong statements regarding their exact nature, other more indirect ways have been used to further elucidate their possible etiology and clinical relevance, several of which have been described in Part II of this thesis. From these studies, two important findings regarding the nature and possible consequence of vessel wall lesions for clinical practice can be distilled. 
First, intracranial vessel wall lesions as seen at 7T MRI in patients with previous ischemic stroke or transient ischemic attack (TIA) are associated with several well-known cardiovascular risk factors, such as increasing age, hypertension and diabetes mellitus (Chapter 5), suggesting that these lesions may indeed represent mild to moderate stages of intracranial atherosclerosis. These findings are comparable with those of a larger community-based 3T vessel wall MRI study - although the study populations of these studies differed - and similar results were seen in a 7T MRI study among patients with a history of (mainly non-cerebral) vascular disease. ${ }^{37-39}$ Interestingly, in all three studies no association was found between vessel wall lesions and smoking, while varying associations were seen with the presence of hyperlipidemia. Possible explanations may be the relatively low number of participants, a difference in association between high-risk and low-risk cardiovascular status of participants, different ethnic backgrounds of participants, or a difference in risk factors between extracranial and intracranial arteries, although the latter is not supported by comparative studies. $38,40-43$ Finally, due to the arbitrarily chosen cutoff value of vessel wall thickening and lack of histopathological validation, some detected vessel wall lesions may be less clinically relevant or represent normal fluctuations of vessel wall thickness, thereby underestimation the true effect. Nevertheless, in all three studies - with population-based, cardiovascular compromised and symptomatic participants clear associations were found, suggesting a true relationship between intracranial vessel wall lesions and cardiovascular risk factors.

Second, these same intracranial vessel wall lesions were associated with cerebral parenchymal (vascular) damage; more specifically, with the presence of any type of infarct and of periventricular white matter hyperintensities (Chapter 6). Interestingly, the types of parenchymal damage with which an association was found, are generally linked to small vessel disease (SVD), not to large vessel atherosclerosis. These findings not only suggest that vessel wall lesions could be clinically relevant, but also implies a relationship between large vessel wall changes and (manifestations of) small vessel disease and/or that these vessel wall changes occur simultaneously. Currently, it remains to be further elucidated whether and in which way these associations exist. Some studies have proposed connecting mechanisms, such as the 'parent artery atheroma theory' in which atherosclerotic plaques in parent arteries cause an occlusion at the orifices of smaller branching arteries resulting in parenchymal damage, while others describe large artery disease and SVD as different entities. ${ }^{44-47}$ Direct comparison of these studies is often difficult because of differences in used definitions and imaging methods for large artery disease, such as total lesion burden (varying form early to endstage atherosclerosis) or solely stenotic lesions, and SVD, with a variety of possible parenchymal manifestations. ${ }^{48-52}$ Larger studies with more uniform measurements and outcomes may contribute to further understanding the potential relationship 
between the larger and smaller intracranial arteries that - although they differ on an anatomical and pathophysiological level - comprise one vessel bed that is physiologically connected.

\section{Implementing intracranial vessel wall MRI in clinical decision-making}

Considering several remaining technical and clinical challenges, intracranial vessel wall MRI could have tremendous potential for neurovascular imaging, by directly visualizing vessel wall pathology and not just the lumen. As mounting evidence suggests a causative relation between intracranial vessel wall lesions and cerebral (vascular) damage like ischemic stroke, the question arises what role vessel wall MRI should have in the clinical diagnostic process and how this may help patients in a clinical setting. ${ }^{53}$ Currently, intracranial vessel wall MRI can function as a diagnostic tool to assess the actual status of the intracranial vessel wall, i.e., the (longitudinal) result of the complete set of risk factors on the vessel wall. Consequently, it could be used for individual risk assessment of intracranial atherosclerosis and in the future may guide patient-tailored (medical) treatment for preventive cerebrovascular management. It might also aid in determining the cause of ischemic stroke, especially in young patients or in those with a previously undetermined cause of ischemic stroke (or TIA): a recent study has shown that the conceived cause of ischemic stroke or TIA is often adapted when applying intracranial vessel wall MRI. ${ }^{54-56}$ Furthermore, ongoing efforts to visualize atherosclerotic plaque components - like calcifications, intraplaque hemorrhage (IPH), lipid core and fibrous caps - to identify predictors of atherosclerotic plaque vulnerability could aid in deciding treatment aggressiveness. ${ }^{2,21,24,28,57-59}$ Currently, most data on vulnerable plaques stem from carotid artery imaging studies and ex vivo intracranial atherosclerotic plaque characterization studies, as in vivo spatial resolution of intracranial vessel wall MRI sequences is often too low for accurate delineation of these individual components. . $4,60,61^{\text {However, recently }}$ several studies have shown examples of signal intensity differences within vessel wall lesions that potentially reflect these individual plaque components, albeit without histopathological validation of these signal intensity differences. ${ }^{2,21,57,58,62-64}$ A meta-analysis that included only intracranial vessel wall MRI studies showed that contrast enhancement of the atherosclerotic plaque, arterial remodeling and plaque irregularity were associated with a higher risk of stroke; remarkably, wellestablished vulnerability predictors for extracranial carotid plaques such as IPH or large lipid core were not associated with vulnerability. ${ }^{58}$ These studies suggest a potential role of intracranial vessel wall MRI in predicting future cerebral ischemic events; nonetheless, the varying results, mostly based on small sample sizes and retrospective designs, currently preclude systematically applied in vivo plaque characterization in clinical practice. ${ }^{1,2,58}$ 
Aparticularsubfield in intracranialatherosclerosisthatmayinfluenceatherosclerotic burden assessment in clinical practice is intra-arterial thrombectomy (and other intraluminal procedures). Several studies have shown evidence of vessel wall changes after intra-arterial treatment (IAT). ${ }^{65-67}$ Stent-retrievers cause mechanical sheer stress when the deployed stent with the trapped culprit thrombus is retracted, while thrombosuction devices use negative pressure to aspirate the culprit thrombus; both exert forces that might be detrimental to the vessel wall. ${ }^{66-}$ ${ }^{68}$ Histopathological studies showed that depending on the type of device used, the vessel wall was more or less affected after the procedure, mostly favoring for thrombosuction devices. ${ }^{66,67}$ Using $7 \mathrm{~T}$ intracranial vessel wall MRI, vessel wall enhancement after thrombosuction was seen more often than in a control group without IAT, and mostly ipsilateral to the occlusion site (Chapter 7). ${ }^{69}$ This is in line with another 7T MRI study investigating the effect of stent-retriever devices on the intracranial vessel wall, ${ }^{70}$ and with several $3 \mathrm{~T}_{\mathrm{MRI}}$ studies..$^{71-74}$ All these studies show comparable results in the detection of post-endovascular vessel wall changes. Although the possibility that IAT devices cause (transient or permanent) changes to the intracranial vessel wall, it will likely not affect their use in the acute setting. It is important to become familiar with these radiological findings as this type of treatment is performed with increasing frequency. ${ }^{75}$ Nonetheless, additional insight in the persistence of these vessel wall changes and their possible risk for future complications (e.g., dissections or re-stenosis) may aid in device optimization and in future may change the indications for follow-up imaging on an individual-patient level.

Besides intracranial atherosclerosis (and iatrogenic complications thereof), intracranial vessel wall MRI has the potential to correctly diagnose many other intracranial vasculopathies that may be less common, and therefore less investigated than atherosclerosis, but that pose a bigger clinical challenge because they often mimic each other on lumenographic imaging studies. ${ }^{1,2,4}$ Actually, in clinical practice the most common indication to use intracranial vessel wall MRI is not assessment of atherosclerotic burden, but diagnosing possible cerebral vasculitis. ${ }^{4}$ Several studies have shown the additional benefit of vessel wall MRI in different types of vasculitis, such as herpes zoster, tuberculous or radiation-induced vasculitis. ${ }^{76-79}$ Although differentiating between these individual types on vessel wall MRI is difficult, the typical enhancement pattern may help the radiologist differentiating vasculitis from other vasculopathies, like reversible vasoconstriction syndrome (RVCS) and intracranial atherosclerosis that require a completely different treatment approach. ${ }^{48,80,81}$ Also, the treatment effect after steroid therapy for vasculitis can be evaluated with a decrease in vessel wall enhancement as a measure of success. ${ }^{4}$ Intracranial aneurysms are another example for which emerging diagnostic evidence points to a significant role of intracranial vessel wall MRI: the presence or absence of aneurysm wall 
enhancement may differentiate between unstable and stable aneurysms, which could guide their therapeutic management. ${ }^{15,82,83}$ Other examples of vessel wall pathology and vasculopathy-associated diseases in which intracranial vessel wall MRI has been suggested to be helpful are dissections, moyamoya disease, Fabry disease, giant cell arteritis, and cerebral amyloid angiopathy; however, sample sizes are small and the additional value of vessel wall MRI in their management remains to be seen. ${ }^{2,5,84-87}$

\section{Future perspectives}

Considering the high demands in SNR, CNR and spatial resolution of intracranial vessel wall MRI and the generally limited clinically applicable acquisition times, further technical developments in sequence design will be inevitable. Next to methods that shorten acquisition times as discussed above and in Chapter 3, new and promising CSF suppression techniques such as delay alternating with nutation for tailored excitation (DANTE), double inversion recovery, antidriven-equilibrium and motion-sensitizing preparation pulses may improve vessel wall delineation by increasing CNR. ${ }^{4,8-10,88-90}$ In addition, recent improvements in hardware, such as a higher number of receiver channels and extended coil sizes, may open up new avenues for clinical application by enabling visualization of both extracranial and intracranial arteries in a single session, providing a complete assessment of both cervical and brain vasculature. ${ }^{14,91,92}$ As these and other technical developments follow each other rapidly, minimal technical requirements and assessment of intracranial vessel wall MRI change at a similar pace, hindering clear guidelines on how to perform intracranial vessel wall MRI in clinical practice. Nevertheless, although the ideal trade-off in sequence parameters may depend on the clinical question, and newer techniques may overrule existing sequence performances and image quality, some general uniform agreements on technique and image assessment are of utmost importance for efficient clinical implementation and setup of comparison studies. A few manuscripts addressing this implementation issue (e.g., Chapter 3 ) have recently been published; however, most studies to date have single-center experience and a limited sample size, thereby limiting translation of their protocols to generally applicable clinical guidelines. , $^{1,2,4,8}$ In addition, there are no standardized scoring systems that can be applied for structural or segmental assessment of the intracranial arterial vessel wall. Some approaches include individual assessment of all large intracranial arteries, further subdivided into segments: ${ }^{4,32,93}$ however, these approaches do not include individual lesion severity (e.g., level of stenosis or plaque vulnerability) nor do they result in a clinically validated 'final vessel wall score' that is linked to practical implications. Also, these approaches are time-consuming and less feasible for assessment of inflammatory vasculopathies in which the identification of typical enhancement patterns not limited to one vessel segment is often more important. ${ }^{4}$ 
Increasing efforts toward strong (international) collaborations among research centers and hospitals would greatly benefit clinical acceptance of intracranial vessel wall MRI. Collaborative networks enable recruitment of larger samples (especially when investigating rare vasculopathies), while image protocols and structural image assessment can be matched across centers, facilitating sound comparisons (for example between different populations), in order to improve the understanding of vessel wall diseases using intracranial vessel wall MRI. Another significant advantage of international collaborations is the setup of larger studies and randomized controlled trials (RCTs), which are currently unavailable but crucial for answering questions regarding the relation between vessel wall findings and clinical outcome (for example risk of future cerebrovascular disease). These studies can also control for potential confounders and identify predictors with higher confidence. ${ }^{1}$ Further, follow-up intracranial vessel wall MRI studies will become more prevalent, increasing knowledge of lesion evolution over time (with or without preventive management) or persistence of vessel wall changes after intraluminal procedures, such as IAT. ${ }^{69}$

\section{Conclusions}

Intracranial vessel wall MRI has changed our approach on how to visualize intracranial arterial pathology, and could have a significant impact on the management of cerebrovascular disease. Although image quality currently does not suffice for quantitative measurements, qualitative assessment has been proven successful in diagnosing vessel wall lesions and differentiating between major vessel wall pathologies. The work described in this thesis has provided evidence that suggests that focal intracranial vessel wall lesions truly represent atherosclerotic lesions, and that they could indeed be clinically relevant. Using intracranial vessel wall MRI together with anatomical MRI sequences further enables assessment of the actual status of the intracranial (and possibly extracranial) vessel wall combined with cerebral parenchymal sequelae in one session. Next steps to advance clinical translation will be (1) true histopathological validation to unravel the true nature of vessel wall lesions; (2) the setup of larger studies to identify the exact associations, predictors and potential confounders to put these lesions in clinical perspective; and (3) international collaborations and consensus guidelines for uniform acquisition and assessment of vessel wall MR images. Ultimately, intracranial vessel wall MRI has the potential to be implemented in the workup and follow-up of patients with cerebrovascular disease and in risk management, using it as non-invasive diagnostic tool to guide patient-tailored preventive and therapeutic decision-making. 


\section{References}

1. Song JW, Guiry SC, Shou H, et al. Qualitative Assessment and Reporting Quality of Intracranial Vessel Wall MR Imaging Studies: A Systematic Review. AJNR American Journal of Neuroradiology 2019;40:2025-32.

2. Mandell DM, Mossa-Basha M, Qiao Y, et al. Intracranial Vessel Wall MRI: Principles and Expert Consensus Recommendations of the American Society of Neuroradiology. AJNR American Journal of Neuroradiology 2017;38:218-229.

3. Bhogal P, Navaei E, Makalanda HL, et al. Intracranial vessel wall MRI. Clinical Radiology 2016;71:293-303.

4. Lindenholz A, van der Kolk AG, Zwanenburg JJM, Hendrikse J. The Use and Pitfalls of Intracranial Vessel Wall Imaging: How We Do It. Radiology 2018;286:12-28.

5. Kern KC, Liebeskind DS. Vessel Wall Imaging of Cerebrovascular Disorders. Curr Treat Options Cardiovasc Med 2019;21:65.

6. De Havenon A, Chung L, Park M, Mossa-Basha M. Intracranial vessel wall MRI: a review of current indications and future applications. Neurovascular Imaging 2016;2:10..

7. Dieleman N, van der Kolk AG, Zwanenburg JJ, et al. Imaging intracranial vessel wall pathology with magnetic resonance imaging: current prospects and future directions. Circulation 2014;130:192-201.

8. Song JW, Moon BF, Burke MP, et al. MR Intracranial Vessel Wall Imaging: A Systematic Review. Journal of Neuroimaging :2020;30:428-442.

9. Lindenholz A, Harteveld AA, Zwanenburg JJM, Siero JCW, Hendrikse J. Comparison of 3T Intracranial Vessel Wall MRI Sequences. AJNR American Journal of Neuroradiology 2018;39:1112-1120.

10. Guggenberger K, Krafft AJ, Ludwig $\mathrm{U}$, et al. High-resolution Compressed-sensing T1 Black-blood MRI : A New Multipurpose Sequence in Vascular Neuroimaging? Clinical Neuroradiology 2019.

11. Li B, Li H, Li J, et al. Relaxation enhanced compressed sensing three-dimensional blackblood vessel wall MR imaging: Preliminary studies. Magnetic Rresonance Imaging 2015;33:932-8.

12. Wang J, Helle M, Zhou Z, Bornert $P$, Hatsukami TS, Yuan C. Joint blood and cerebrospinal fluid suppression for intracranial vessel wall MRI. Magnetic Resonance in Medicine 2016;75:831-8.

13. Viessmann O, Li L, Benjamin P, Jezzard P. T2-Weighted intracranial vessel wall imaging at 7 Tesla using a DANTE-prepared variable flip angle turbo spin echo readout (DANTESPACE). Magnetic Resonance in Medicine 2017;77:655-663.

14. Hu X, Li Y, Zhang L, Zhang X, Liu X, Chung YC. A 32-channel coil system for MR vessel wall imaging of intracranial and extracranial arteries at 3T. Magnetic Resonance Imaging 2017;36:86-92. 
15. Larsen N, von der Brelie C, Trick D, et al. Vessel Wall Enhancement in Unruptured Intracranial Aneurysms: An Indicator for Higher Risk of Rupture? High-Resolution MR Imaging and Correlated Histologic Findings. AJNR American Journal of Neuroradiology 2018;39:1617-21.

16. Kleinloog R, Korkmaz E, Zwanenburg JJ, et al. Visualization of the aneurysm wall: a 7.0-tesla magnetic resonance imaging study. Neurosurgery 2014;75:614-22.

17. Miller DV, Salvarani C, Hunder GG, et al. Biopsy findings in primary angiitis of the central nervous system. Am J Surg Pathol 2009;33:35-43.

18. Zeiler SR, Qiao Y, Pardo CA, Lim M, Wasserman BA. Vessel Wall MRI for Targeting Biopsies of Intracranial Vasculitis. AJNR American Journal of Neuroradioly 2018;39:2034-6.

19. Turan TN, Rumboldt Z, Granholm AC, et al. Intracranial atherosclerosis: correlation between in-vivo 3T high resolution MRI and pathology. Atherosclerosis 2014;237:460-3.

20. Harteveld A. In vivo and ex vivo vessel wall MRI of the circle of Willis. PhD thesis, Utrecht University The Netherlands 2017:2-212.

21. Yang WJ, Wong KS, Chen XY. Intracranial Atherosclerosis: From Microscopy to HighResolution Magnetic Resonance Imaging. Journal of Stroke 2017;19:249-60.

22. Chen $\mathrm{XY}$, Wong KS, Lam WW, Zhao HL, Ng HK. Middle cerebral artery atherosclerosis: histological comparison between plaques associated with and not associated with infarct in a postmortem study. Cerebrovasc Dis 2008;25:74-80.

23. Van der Kolk AG, Zwanenburg JJ, Denswil NP, et al. Imaging the intracranial atherosclerotic vessel wall using 7T MRI: initial comparison with histopathology. AJNR American Journal of Neuroradiology 2015;36:694-701.

24. Harteveld AA, Denswil NP, Siero JC, et al. Quantitative Intracranial Atherosclerotic Plaque Characterization at 7T MRI: An Ex Vivo Study with Histologic Validation. AJNR American Journal of Neuroradiology 2016;37:802-10.

25. Yang WJ, Chen XY, Zhao HL, et al. Postmortem Study of Validation of Low Signal on Fat-Suppressed T1-Weighted Magnetic Resonance Imaging as Marker of Lipid Core in Middle Cerebral Artery Atherosclerosis. Stroke 2016;47:2299-304.

26. Majidi S, Sein J, Watanabe M, et al. Intracranial-derived atherosclerosis assessment: an in vitro comparison between virtual histology by intravascular ultrasonography, 7T MRI, and histopathologic findings. AJNR American Journal of Neuroradiology 2013;34:2259-64.

27. Lopez Gonzalez MR, Foo SY, Holmes WM, et al. Atherosclerotic Carotid Plaque Composition: A 3T and 7T MRI-Histology Correlation Study. Journal of Neuroimaging 2016;26:406-13.

28. Ritz K, Denswil NP, Stam OC, van Lieshout J, Daemen MJ. Cause and mechanisms of intracranial atherosclerosis. Circulation 2014;130:1407-14.

29. Harteveld AA, van der Kolk AG, van der Worp HB, et al. High-resolution intracranial vessel wall MRI in an elderly asymptomatic population: comparison of 3T and 7T. European Radiology 2017;27:1585-1595. 
30. Harteveld AA, van der Kolk AG, van der Worp HB, et al. Detecting Intracranial Vessel Wall Lesions With 7T-Magnetic Resonance Imaging: Patients With Posterior Circulation Ischemia Versus Healthy Controls. Stroke 2017;48:2601-4.

31. Cogswell PM, Lants SK, Davis LT, Donahue MJ. Vessel wall and lumen characteristics with age in healthy participants using 3T intracranial vessel wall magnetic resonance imaging. Journal of Magnetic Resonance Imaging : JMRI 2019;50:1452-60.

32. Van der Kolk AG, Zwanenburg JJ, Brundel M, et al. Distribution and natural course of intracranial vessel wall lesions in patients with ischemic stroke or TIA at 7.0 Tesla MRI. European Radiology 2015;25:1692-700.

33. Gutierrez J, Elkind MS, Petito C, Chung DY, Dwork AJ, Marshall RS. The contribution of HIV infection to intracranial arterial remodeling: a pilot study. Neuropathology 2013;33:256-63.

34. Qiao Y, Guallar E, Suri FK, et al. MR Imaging Measures of Intracranial Atherosclerosis in a Population-based Study. Radiology 2016:151124.

35. Van Hespen KM, Zwanenburg JJM, Harteveld AA, Luijten PR, Hendrikse J, Kuijf HJ. Intracranial Vessel Wall Magnetic Resonance Imaging Does Not Allow for Accurate and Precise Wall Thickness Measurements: An Ex Vivo Study. Stroke 2019;50:e283-e4.

36. Bouvy WH, Biessels GJ, Kuijf HJ, Kappelle LJ, Luijten PR, Zwanenburg JJ. Visualization of perivascular spaces and perforating arteries with $7 \mathrm{~T}$ magnetic resonance imaging. Investigative Radiology 2014;49:307-13.

37. Lindenholz A, van der Kolk AG, van der Schaaf IC, et al. Intracranial Atherosclerosis Assessed with 7-T MRI: Evaluation of Patients with Ischemic Stroke or Transient Ischemic Attack. Radiology 2020;295:162-70.

38. Qiao Y, Suri FK, Zhang Y, et al. Racial Differences in Prevalence and Risk for Intracranial Atherosclerosis in a US Community-Based Population. JAMA Cardiology 2017;12:13411348.

39. Zwartbol MHT, van der Kolk AG, Ghaznawi R, et al. Intracranial Vessel Wall Lesions on 7T MRI (Magnetic Resonance Imaging). Stroke 2019;50:88-94.

40. Gorelick P, Wong KS, Liu L. Epidemiology. Frontiers of Neurology and Neuroscience 2016;40:34-46.

41. Arenillas JF, Lopez-Cancio E, Wong KS. Biomarkers, Natural Course and Prognosis. Frontiers of Neurology and Neuroscience 2016;40:93-108.

42. Zwartbol MHT, Geerlings MI, Ghaznawi R, Hendrikse J, van der Kolk AG, Group U-SS. Intracranial Atherosclerotic Burden on 7T MRI Is Associated with Markers of Extracranial Atherosclerosis: The SMART-MR Study. AJNR American Journal of Neuroradiology 2019;40:2016-22.

43. Bang OY, Chung JW, Ryoo S, et al. Brain microangiopathy and macroangiopathy share common risk factors and biomarkers. Atherosclerosis 2016;246:71-7.

44. Shi Y, Wardlaw JM. Update on cerebral small vessel disease: a dynamic whole-brain disease. Stroke and Vascular Neurology 2016;1:83-92. 
45. Kong Q, Zhang Z, Yang Q, et al. 7T TOF-MRA shows modulated orifices of lenticulostriate arteries associated with atherosclerotic plaques in patients with lacunar infarcts. European Journal of Radiology 2019;118:271-6.

46. Aboyans V, Lacroix $\mathrm{P}$, Criqui $\mathrm{MH}$. Large and small vessels atherosclerosis: similarities and differences. Progress in Cardiovascular Diseases 2007;50:112-25.

47. Kwon HM, Lynn MJ, Turan TN, et al. Frequency, Risk Factors, and Outcome of Coexistent Small Vessel Disease and Intracranial Arterial Stenosis: Results From the Stenting and Aggressive Medical Management for Preventing Recurrent Stroke in Intracranial Stenosis (SAMMPRIS) Trial. JAMA Neurology 2016;73:36-42.

48. Dieleman N, van der Kolk AG, van Veluw SJ, et al. Patterns of intracranial vessel wall changes in relation to ischemic infarcts. Neurology 2014;83:1316-20.

49. Boulouis G, Charidimou A, Auriel E, et al. Intracranial atherosclerosis and cerebral small vessel disease in intracerebral hemorrhage patients. Journal of the Neurological Sciences 2016;369:324-9.

50. Brisset M, Boutouyrie P, Pico F, et al. Large-vessel correlates of cerebral small-vessel disease. Neurology 2013;80:662-9.

51. Wardlaw JM, Smith EE, Biessels GJ, et al. Neuroimaging standards for research into small vessel disease and its contribution to ageing and neurodegeneration. The Lancet Neurology 2013;12:822-38.

52. Dieleman N, van der Kolk AG, Zwanenburg J, et al. Relations between location and type of intracranial atherosclerosis and parenchymal damage. Journal of Cerebral Blood Flow and Metabolism 2016;36:1271-80.

53. Wang Y, Liu X, Wu X, Degnan AJ, Malhotra A, Zhu C. Culprit intracranial plaque without substantial stenosis in acute ischemic stroke on vessel wall MRI: A systematic review. Atherosclerosis 2019;287:112-21.

54. Kesav P, Krishnavadana B, Kesavadas C, et al. Utility of intracranial high-resolution vessel wall magnetic resonance imaging in differentiating intracranial vasculopathic diseases causing ischemic stroke. Neuroradiology 2019;61:389-96.

55. McCarty JL, Leung LY, Peterson RB, et al. Ischemic Infarction in Young Adults: A Review for Radiologists. Radiographics 2019;39:1629-48.

56. Schaafsma JD, Rawal S, Coutinho JM, et al. Diagnostic Impact of Intracranial Vessel Wall MRI in 205 Patients with Ischemic Stroke or TIA. AJNR American Journal of Neuroradioly 2019;40:1701-6.

57. Xu WH, Li ML, Gao S, et al. Middle cerebral artery intraplaque hemorrhage: prevalence and clinical relevance. Annals of Neurology 2012;71:195-8.

58. Lee HN, Ryu CW, Yun SJ. Vessel-Wall Magnetic Resonance Imaging of Intracranial Atherosclerotic Plaque and Ischemic Stroke: A Systematic Review and Meta-Analysis. Frontiers in Neurology 2018;9:1032.

59. Derdeyn CP, Chimowitz MI, Lynn MJ, et al. Aggressive medical treatment with or without stenting in high-risk patients with intracranial artery stenosis (SAMMPRIS): the final results of a randomised trial. Lancet 2014;383:333-41. 
60. Yuan C, Mitsumori LM, Ferguson MS, et al. In vivo accuracy of multispectral magnetic resonance imaging for identifying lipid-rich necrotic cores and intraplaque hemorrhage in advanced human carotid plaques. Circulation 2001;104:2051-6.

61. Yuan C, Mitsumori LM, Beach KW, Maravilla KR. Carotid atherosclerotic plaque: noninvasive MR characterization and identification of vulnerable lesions. Radiology 2001;221:285-99.

62. Chen XY, Wong KS, Lam WW, Ng HK. High signal on T1 sequence of magnetic resonance imaging confirmed to be intraplaque haemorrhage by histology in middle cerebral artery. International Journal of Stroke 2014;9:E19.

63. Turan TN, Bonilha L, Morgan PS, Adams RJ, Chimowitz MI. Intraplaque hemorrhage in symptomatic intracranial atherosclerotic disease. Journal of Neuroimaging 2011;21:e159-61.

64. Turan TN, LeMatty T, Martin R, et al. Characterization of intracranial atherosclerotic stenosis using high-resolution MRI study--rationale and design. Brain and Behavior 2015;5:e00397.

65. Gory B, Bresson D, Kessler I, et al. Histopathologic evaluation of arterial wall response to 5 neurovascular mechanical thrombectomy devices in a swine model. AJNR American Journal of Neuroradiology 2013;34:2192-8.

66. Teng D, Pannell JS, Rennert RC, et al. Endothelial trauma from mechanical thrombectomy in acute stroke: in vitro live-cell platform with animal validation. Stroke 2015;46:1099-106.

67. Peschillo S, Diana F, Berge J, Missori P. A comparison of acute vascular damage caused by ADAPT versus a stent retriever device after thrombectomy in acute ischemic stroke: a histological and ultrastructural study in an animal model. Journal of Neurointerventional Surgery 2017;9:743-749.

68. The penumbra pivotal stroke trial: safety and effectiveness of a new generation of mechanical devices for clot removal in intracranial large vessel occlusive disease. Stroke 2009;40:2761-8.

69. Lindenholz A, van der Schaaf IC, van der Kolk AG, et al. MRI Vessel Wall Imaging after Intra-Arterial Treatment for Acute Ischemic Stroke. AJNR American Journal of Neuroradiology 2020;41:624-31.

70. Truong M, Bloch KM, Andersen M, Andsberg G, Toger J, Wasselius J. Subacute vessel wall imaging at 7-T MRI in post-thrombectomy stroke patients. Neuroradiology 2019;61:1145-53.

71. Power S, Matouk C, Casaubon LK, Silver FL, Krings T, Mikulis DJ. Vessel wall magnetic resonance imaging in acute ischemic stroke: effects of embolism and mechanical thrombectomy on the arterial wall. Stroke; a journal of cerebral circulation 2014;45:2330-4.

72. Hsieh K, Verma RK, Schroth G, et al. Multimodal 3 Tesla MRI confirms intact arterial wall in acute stroke patients after stent-retriever thrombectomy. Stroke 2014;45:3430-2.

73. Abraham P, Scott Pannell J, Santiago-Dieppa DR, et al. Vessel wall signal enhancement on 3-T MRI in acute stroke patients after stent retriever thrombectomy. Neurosurgical focus 2017;42:E20. 
74. Seo WK, Oh K, Suh SI, Seol HY. Clinical Significance of Wall Changes After Recanalization Therapy in Acute Stroke: High-Resolution Vessel Wall Imaging. Stroke 2017:48:10771080.

75. Rodrigues FB, Neves JB, Caldeira D, Ferro JM, Ferreira JJ, Costa J. Endovascular treatment versus medical care alone for ischaemic stroke: systematic review and meta-analysis. BMJ 2016;353:i1754.

76. Cheng-Ching E, Jones S, Hui FK, et al. High-resolution MRI vessel wall imaging in varicella zoster virus vasculopathy. Journal of the Neurological Sciences 2015;351:168-73.

77. Chen H, Li X, Zhang X, et al. Late delayed radiation-induced cerebral Arteriopathy by highresolution magnetic resonance imaging: a case report. BMC neurology 2019;19:232.

78. Song JW, Lehman L, Rivkin M, Gorman MP, Yang E. Serial vessel wall MR imaging of pediatric tuberculous vasculitis. Neurol Clin Pract 2019;9:459-61.

79. Song JW, Ojeda S, Romero JM. High resolution vessel wall MRI and vasculopathy related to herpes zoster ophthalmicus. Clin Imaging 2018;50:336-9.

80. Mandell DM, Matouk CC, Farb RI, Krings T, Agid R, Brugge K. Vessel wall MRI to differentiate between reversible cerebral vasoconstriction syndrome and central nervous system vasculitis: preliminary results. Stroke 2012;43:860-2.

81. Obusez EC, Hui F, Hajj-Ali RA, Cerejo R, Calabrese LH, Hammad T. High-resolution MRI vessel wall imaging: spatial and temporal patterns of reversible cerebral vasoconstriction syndrome and central nervous system vasculitis. AJNR American Journal of Neuroradiology 2014;35:1527-32.

82. Texakalidis P, Hilditch CA, Lehman V, Lanzino G, Pereira VM, Brinjikji W. Vessel Wall Imaging of Intracranial Aneurysms: Systematic Review and Meta-analysis. World Neurosurgery 2018;117:453-8 e1.

83. Wang X, Zhu C, Leng Y, Degnan AJ, Lu J. Intracranial Aneurysm Wall Enhancement Associated with Aneurysm Rupture: A Systematic Review and Meta-analysis. Academic Radiology 2019;26:664-73.

84. Ryoo S, Cha J, Kim SJ, et al. High-resolution magnetic resonance wall imaging findings of Moyamoya disease. Stroke 2014;45:2457-60.

85. Kong DZ, Lian YH, Wang LJ, Wang CM, Meng YY, Zhou HW. Central nervous system vasculopathy caused by Fabry disease: a case report. BMC neurology 2019;19:115.

86. Correa DG, de Oliveira ESDG, da Cruz LCH, Jr. Use of high-resolution vessel wall magnetic resonance imaging in the diagnosis of temporal arteritis. Rheumatology International 2019;39:1479-81.

87. Hao Q, Tsankova NM, Shoirah H, Kellner CP, Nael K. Vessel Wall MRI Enhancement in Noninflammatory Cerebral Amyloid Angiopathy. AJNR American Journal of Neuroradiology 2020;41:446-8.

88. Xie Y, Yang Q, Xie G, Pang J, Fan Z, Li D. Improved black-blood imaging using DANTESPACE for simultaneous carotid and intracranial vessel wall evaluation. Magnetic Resonance in Medicine 2016;75:2286-94. 
89. Yang H, Zhang X, Qin Q, Liu L, Wasserman BA, Qiao Y. Improved cerebrospinal fluid suppression for intracranial vessel wall MRI. Journal of Magnetic Resonance Imaging : JMRI 2016.

90. Wang J, Yarnykh VL, Yuan C. Enhanced image quality in black-blood MRI using the improved motion-sensitized driven-equilibrium (iMSDE) sequence. Journal of Magnetic Resonance Imaging : JMRI 2010;31:1256-63.

91. Jia S, Zhang L, Ren L, et al. Joint intracranial and carotid vessel wall imaging in 5 minutes using compressed sensing accelerated DANTE-SPACE. European Radiology 2020;30: 119-27.

92. Wan L, Zhang N, Zhang L, et al. Reproducibility of simultaneous imaging of intracranial and extracranial arterial vessel walls using an improved T1-weighted DANTE-SPACE sequence on a 3T MR system. Magn Reson Imaging 2019;62:152-8.

93. Dieleman N. MR imaging of intracranial vessel wall pathologies and parenchymal brain damage. PhD thesis, Utrecht University, The Netherlands 2016:2-176: 
General Discussion 
Appendices 


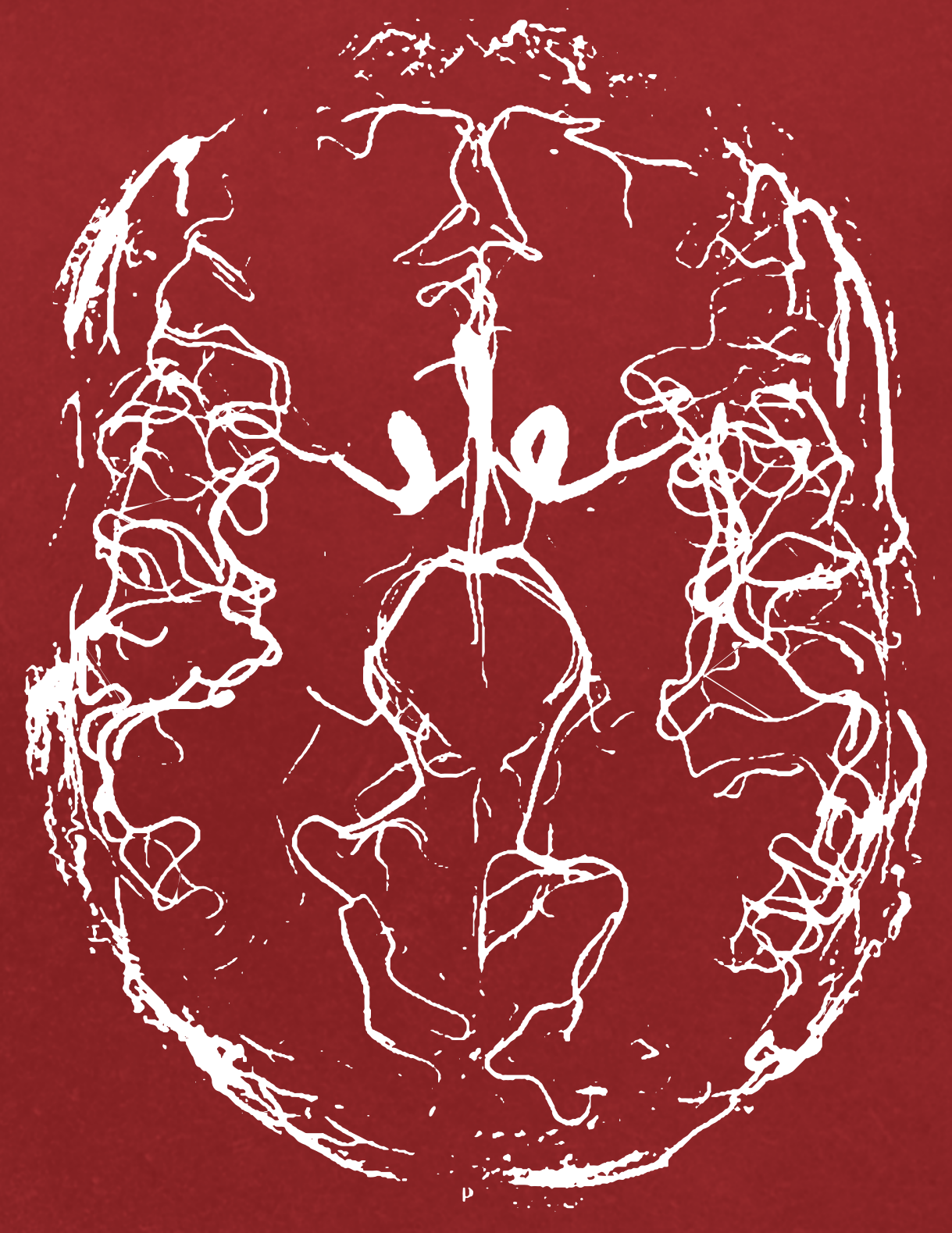




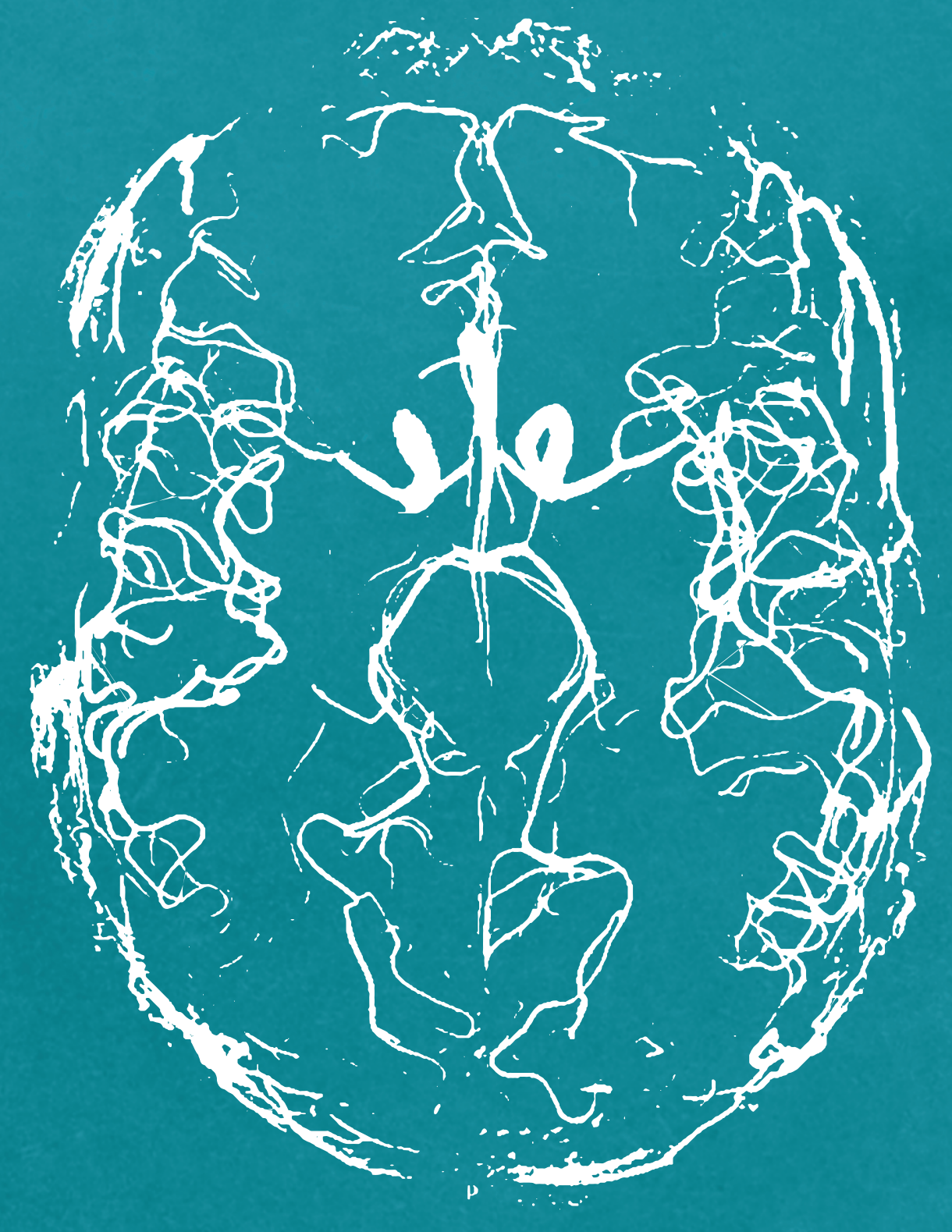


ADDENDUM I

\section{Dutch Summary}

Nederlandse Samenvatting 
De afgelopen twee decennia kenden vele technologische ontwikkelingen op het gebied van MRI van de arteriële vaatwand. Terwijl initieel enkel de extracraniële arteriën in de hals (carotiden) in beeld konden worden gebracht, zijn er tegenwoordig - door zowel technische verbeteringen als toegenomen signaalruisverhouding (SNR), beeldcontrast (CNR) en spatiële resolutie door de komst van hogere magneetveldsterkten (3T en 7T) - ook meerdere MRI-sequenties beschikbaar voor het visualiseren van de intracraniële arteriën. Deze sequenties kunnen worden gebruikt om de submillimeter-dunne intracraniële vaatwand te beoordelen op afwijkingen. Het gebruik hiervan in klinische studies heeft inmiddels geleid tot de definitie van meerdere wandkarakteristieken, kenmerkend voor een verscheidenheid aan vasculaire aandoeningen. Het is echter lastig om histopathologische validatie te verkrijgen van vaatwandafwijkingen zoals gezien op MRI-beelden. Een definitief antwoord op de vraag of het ook daadwerkelijk is wat we denken te zien (de aard van de afwijkingen), is hierdoor tot dusver uitgebleven. Daarnaast zijn er weinig studies verricht naar de (aanvullende) klinische waarde van de gedetecteerde vaatwandafwijkingen. Deze onzekerheden hebben tot op heden een belangrijke rem geplaatst op de klinische translatie en hiermee gepaard gaande systematische implementatie in de klinische diagnostiek.

In het eerste deel van dit proefschrift wordt aandacht besteed aan de technische aspecten en uitdagingen van MRI van de intracraniële vaatwand in relatie tot veldsterkte en acquisitietijd. Hoofdstuk 2 geeft een overzicht van de voordelen en uitdagingen van 7T MRI bij de diagnostiek van vasculaire neurologische aandoeningen, waaronder cerebrale ischemie. In hoofdstuk 3 wordt toegespitst op de verschillen, overeenkomsten en algemene uitdagingen van MRI van de intracraniële vaatwand, zowel op 7T als op 3T. Hoewel 3T vanuit klinisch perspectief het meest praktisch is, beelden van de vaatwand kan verkrijgen met hoge resolutie en kan helpen in de differentiatie tussen verschillende vasculaire aandoeningen, zijn de vaatwanden op 7T duidelijker af te grenzen door hogere CNR en door betere onderdrukking van cerebrospinale vloeistof (CSF). Hierdoor is 7T sensitiever bij de detectie van vaatwandafwijkingen. Daarnaast biedt hoofdstuk 3 radiologen handvatten voor de beoordeling van vaatwandscans, waaronder standaard beoordeling van de gezonde vaatwand, valkuilen bij de beoordeling, het beschrijven van afwijkingen en de interpretatie in een klinische context. In hoofdstuk 4 wordt de lange acquisitietijd van veel intracraniële vaatwandscans onder de loep genomen. Dit is een gevolg van de hoge technische eisen die aan deze sequenties worden gesteld. Door aanpassingen in de spatiële resolutie, SNR, CNR of een combinatie van deze factoren is een 3T vaatwandsequentie ontwikkeld die dertig procent sneller is dan de huidige klinisch gebruikte sequentie, met behoud van zowel voldoende kwalitatieve als kwantitatieve beeldkwaliteit. 
Het tweede deel van dit proefschrift wordt gewijd aan het op een indirecte manier nader uitkristalliseren van de aard van vaatwandafwijkingen, zodat een duidelijker beeld ontstaat van de potentiële rol van MRI van de intracraniële vaatwand in het klinisch diagnostisch proces. Hoofdstuk 5 laat zien dat verschillende cardiovasculaire risicofactoren - zoals een hogere leeftijd, diabetes mellitus en hypertensie - geassocieerd zijn met een groter aantal intracraniële vaatwandafwijkingen bij patiënten met een herseninfarct of TIA. In dezelfde patiëntengroep wordt tevens gezien dat een groter aantal intracraniële vaatwandafwijkingen geassocieerd is met de aanwezigheid van schade aan het hersenparenchym, zoals infarcten die vaak worden gezien bij patiënten met small vessel disease (schade aan de kleine hersenvaten) en matig-ernstige periventriculaire wittestofafwijkingen (hoofdstuk 6). De resultaten van deze studies (I) steunen de theorie dat de gevonden vaatwandlaesies inderdaad 'echte' atherosclerotische laesies zijn en niet bijvoorbeeld normale variaties in vaatwanddikte; (II) suggereren een risicoprofiel voor intracraniële vaatwandlaesies die niet veel anders is dan voor extracraniële atherosclerose en die zou kunnen worden aangepakt met risicofactorreductie; en (III) tonen de relatie tussen deze vaatwandafwijkingen en schade van het hersenparenchym. Desalniettemin zullen grotere studies nodig zijn om te bepalen of bijvoorbeeld medische interventie (zoals behandeling met antihypertensiva) ook daadwerkelijk zorgt voor een vermindering van het aantal vaatwandafwijkingen en/of parenchymschade. In hoofdstuk 7 wordt tenslotte MRI van de intracraniële vaatwand ingezet ter beoordeling van eventuele schade na intra-arteriële trombectomie (IAT). Patiënten die zijn behandeld met IAT toonden vaker ipsilaterale (concentrische) vaatwandaankleuring dan patiënten die deze behandeling niet hebben ondergaan. Bij beoordeling van follow-up beeldvorming van deze patiënten zal rekening moeten worden gehouden met deze reactieve veranderingen van de vaatwand na IAT.

Dit proefschrift illustreert de potentiële waarde van MRI van de intracraniële vaatwand in de klinische praktijk. Hierop volgende stappen in de klinische translatie zijn histopathologische validatie van de in beeld gebrachte vaatwandafwijkingen, het opzetten van grotere studies om een beter beeld te krijgen van de relatie tussen vaatwandlaesies, mogelijke risicofactoren en het klinische beeld, en de formatie van internationale samenwerkingsverbanden met als doel de standaardisatie van acquisitieprotocollen en beoordeling van vaatwandscans. Uiteindelijk heeft intracraniële vaatwand MRI de veelbelovende potentie om geïmplementeerd te worden in de work-up en follow-up van patiënten met een cerebrovasculaire aandoening, in risicomanagement en in het diagnosticeren van andere vaatwandziektes, met als doel preventieve en therapeutische beslisvoering te sturen. 


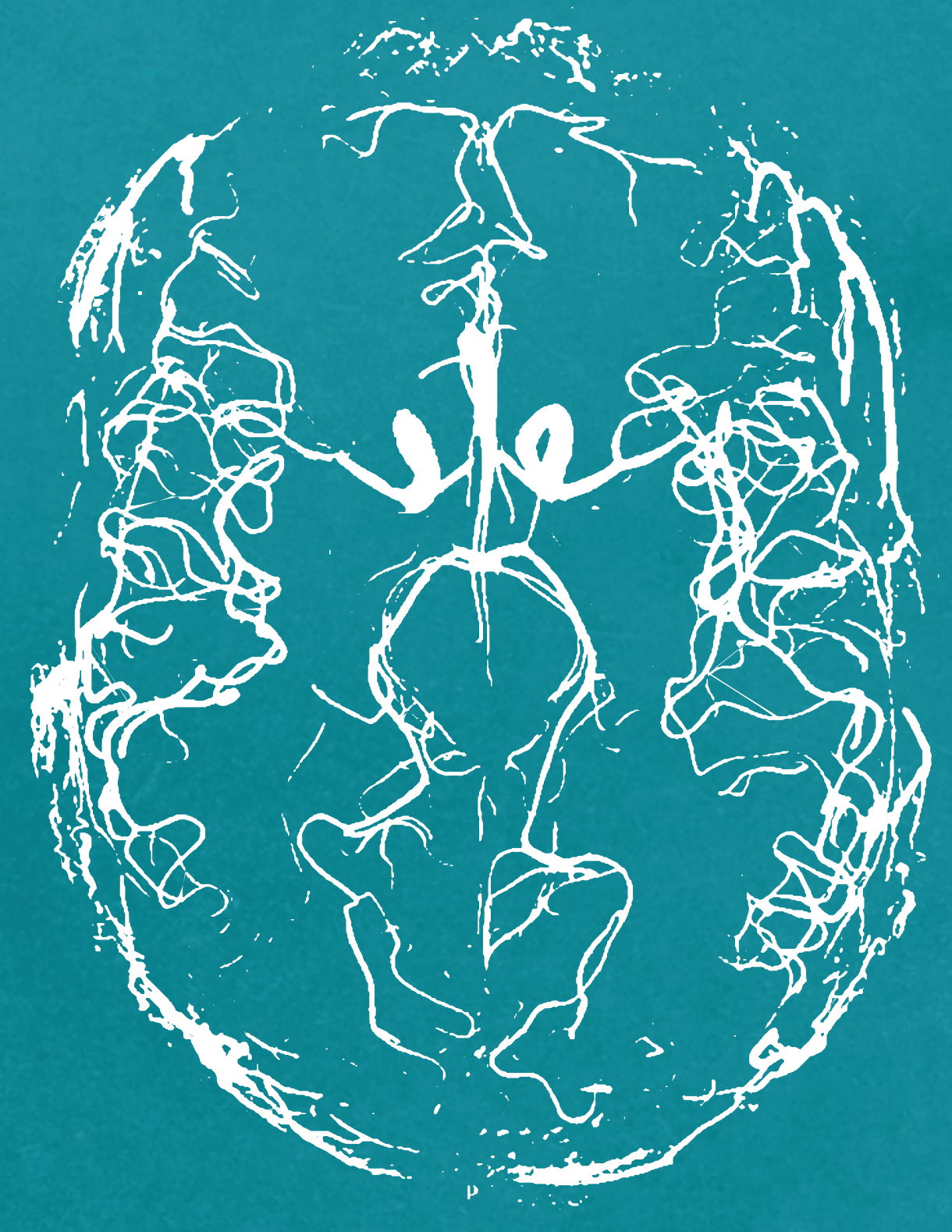


ADDENDUM II

List of Publications 


\section{Published manuscripts}

Lindenholz A, de Bresser J, van der Kolk AG, van der Worp HB, Witkamp TD, Hendrikse J, van der Schaaf IC. Intracranial Atherosclerotic Burden and Cerebral Parenchymal Changes at 7T MRI in Patients with TIA or Ischemic Stroke. Frontiers of Neurology, section Stroke (Research Topic: Cerebrovascular imaging - from micro- to macroscopic scales), 2021.

Versteegh VE, Welvaart WN, Oberink-Gustafsson EEM, Lindenholz A, Schaefer-Prokop CM, Staaks GHA. Non-traumatic Complications of a Solitary Rib Osteochondroma; an Unusual cause of Hemoptysis and Pneumothorax. BJR case reports 2020; 6: 20200015.

Lindenholz A, van der Schaaf IC, van der Kolk AG, van der Worp HB, Harteveld AA, Kappelle LJ, Hendrikse J. MRI Vessel Wall Imaging after Intra-Arterial Treatment for Acute Ischemic Stroke. AJNR American Journal of Neuroradiology 2020; 41:624-31.

Lindenholz A, van der Kolk AG, van der Schaaf IC, van der Worp HB, Harteveld AA, Dieleman $\mathrm{N}$, Bots ML, Hendrikse J. Intracranial Atherosclerosis Assessed with 7-T MRI: Evaluation of Patients with Ischemic Stroke or Transient Ischemic Attack. Radiology 2020; 295:162-70.

Jolink WM, Lindenholz A, van Etten ES, van Nieuwenhuizen KM, Schreuder FH, Kuijf HJ, van Osch MJ, Hendrikse J, Rinkel GJ, Wermer MJ, Klijn CJ. Contrast Leakage Distant from the Hematoma in Patients with Spontaneous ICH: A 7 T MRI study. JCBFM Journal of Cerebral Blood Flow \& Metabolism 2020; 40:1002-11.

Lindenholz A, Harteveld AA, Zwanenburg JJM, Siero JCW, Hendrikse J. Comparison of 3T Intracranial Vessel Wall MRI Sequences. AJNR American Journal of Neuroradiology 2018; Jun;39(6):1112-1120.

Lindenholz A, van der Kolk AG, Zwanenburg JJM, Hendrikse J. The Use and Pitfalls of Intracranial Vessel Wall Imaging: How We Do It. Radiology 2018; Jan;286(1):12-28.

De Cocker LJ, Lindenholz A, ZwanenburgJJ, van der Kolk AG, Zwartbol M, Luijten PR, Hendrikse J. Clinical Vascular Imaging in the Brain at 7T. Neuroimage 2018; Mar;168:452-458.

Lindenholz A, TerBrugge KG, van Dijk JM, Farb RI. The Accuracy and Utility of ContrastEnhanced MR Angiography for Localization of Spinal Dural Arteriovenous Fistulas: the Toronto Experience. European Radiology 2014; Nov;24(11):2885-94.

\section{Non-peer reviewed publications}

Lindenholz A. Comparison of 3T Intracranial Vessel Wall MRI Sequences. American Journal of Neuroradiology (AJNR); News Digest: September-October 2019.

Lindenholz A. MRI Vessel Wall Imaging after Intra-Arterial Treatment for Acute Ischemic Stroke. American Journal of Neuroradiology (AJNR); News Digest: 2020.

\section{Submitted manuscripts}

Dieleman N, van der Kolk AG, Frijns CJM, Harteveld AA, Zwanenburg JJM, Kuijf HJ, Lindenholz A, Kapelle LJ, Luijten PR, Hendrikse J. Vessel Wall Imaging in Cerebral Vasculitis: Diagnostic Value and Treatment Effects at 3T\&7T MRI. 


\section{Conferences Presentations (first author only)}

Lindenholz A, van der Kolk AG, van der Schaaf IC, van der Worp HB, Harteveld AA, Dieleman $\mathrm{N}$, Bots ML, Hendrikse J. Vascular Risk Factors and Intracranial Atherosclerosis at 7T Vessel Wall MRI in Caucasian Ischemic Stroke and TIA patients. ISMRM 26 th annual meeting, Paris, France, 2019.

Lindenholz A, van der Kolk AG, van der Schaaf IC, van der Worp HB, Harteveld AA, Dieleman $\mathrm{N}$, Bots ML, Hendrikse J. Vascular Risk Factors and the Atherosclerotic Lesion Burden at 7T Vessel Wall MRI in Ischemic Stroke and TIA patients. Oral presentation, ISMRM Benelux 2018, Antwerp, Belgium.

Lindenholz A, van der Kolk AG, Zwanenburg JJM, Hendrikse J. The Use and Pitfalls of Intracranial Vessel Wall Imaging: How We Do It. Oral presentation, ImagO Scientific Conference day, 2018.

Lindenholz A, van der Schaaf IC, van der Kolk AG, van der Worp HB, Harteveld AA, Kappelle LJ, Hendrikse J. High-resolution MR Vessel Wall Imaging after Intra-Arterial Treatment for Acute Ischemic Stroke. ISMRM 25th annual meeting, Honolulu, Hawaii, USA, 2017.

Lindenholz A, van der Schaaf IC, van der Kolk AG, van der Worp HB, Harteveld AA, Kappelle LJ, Hendrikse J. MRI Vessel Wall Imaging After Intra-Arterial Treatment for Acute Ischemic Stroke. ISMRM Benelux 2017, Tilburg, Netherlands.

Lindenholz A, Harteveld AA, Zwanenburg JJM, Siero JCW, Hendrikse J. A Multiple Comparison between 3 T Intracranial Vessel Wall Sequences. ISMRM $24^{\text {th }}$ annual meeting, Singapore, 2016.

Lindenholz A, Harteveld AA, Zwanenburg JJM, Siero JCW, Hendrikse J. A Multiple Comparison between 3T Intracranial Vessel Wall Sequences. Oral presentation, ImagO Scientific Conference day, 2016.

\section{Awards}

ISMRM Magna Cum Laude Merit Award. Vascular Risk Factors and Intracranial Atherosclerosis at 7T Vessel Wall MRI in Caucasian Ischemic Stroke and TIA Patients. ISMRM $26^{\text {th }}$ annual meeting, Paris, France.

ISMRM Magna Cum Laude Merit Award. High-resolution MR Vessel Wall Imaging after Intra-Arterial Treatment for Acute Ischemic Stroke. ISMRM 25 th annual meeting, Honolulu, Hawaii, USA.

Nominated for the annual Lucien Levy Best Research Article Award 2020 of the American Journal of Neuroradiology. MRI Vessel Wall Imaging after Intra-Arterial Treatment for Acute Ischemic Stroke. AJNR American Journal of Neuroradiology 2020; 41:624-31. 


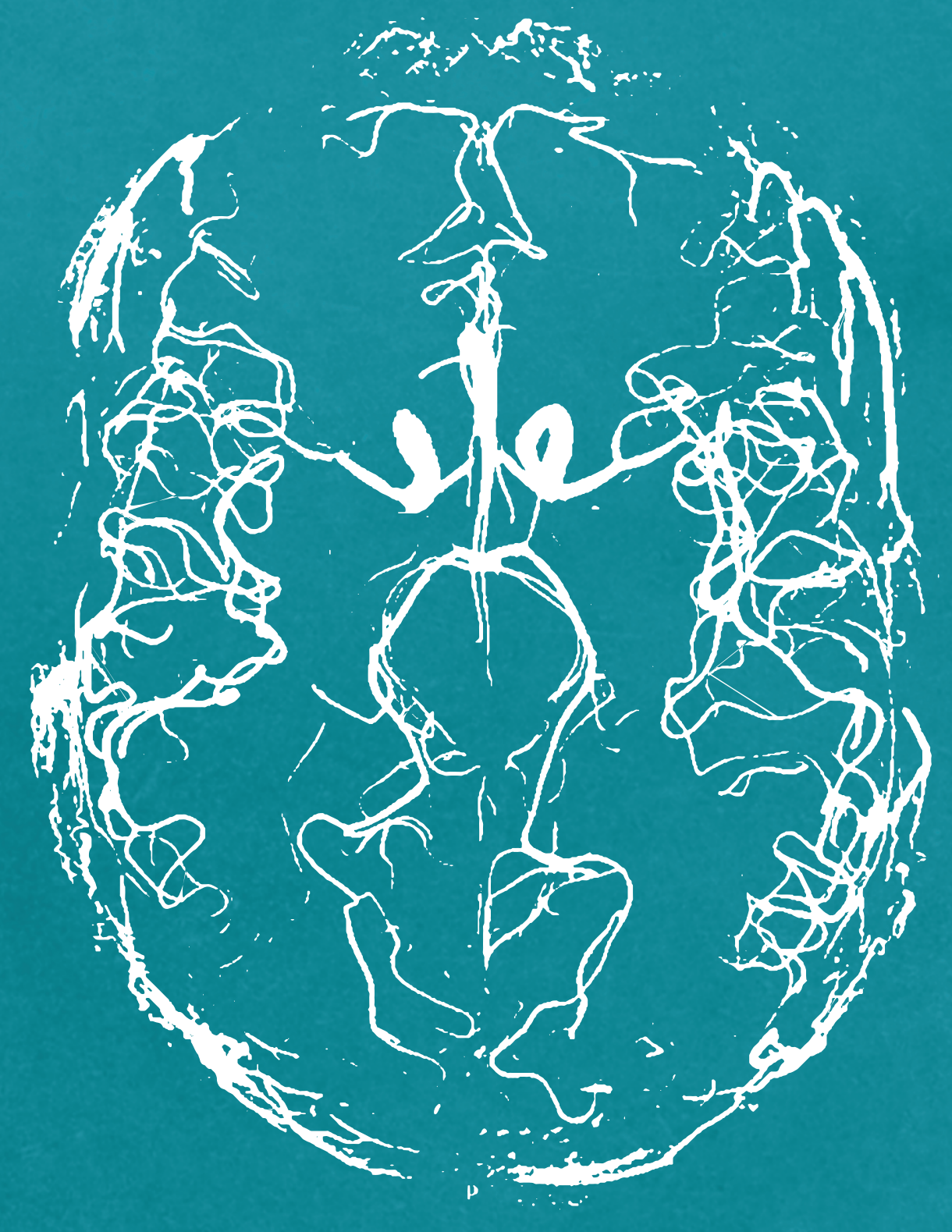


ADDENDUM III

Dankwoord 


\section{Dankwoord}

Finis coronat opus, met andere woorden: het einde bekroont het werk. Na ruim zes jaar onderzoek, gecombineerd met onder andere een master Epidemiologie en mijn specialisatie tot radioloog, komt de finishlijn in zicht. Geen sprint, maar een marathon. Het onderzoek speelde de afgelopen jaren een prominente rol in mijn leven. Ik heb ontzettend veel geleerd en het heeft mij als persoon gevormd. Het voelt dan ook bijzonder om naar de afronding van deze periode over te mogen gaan en iedereen te bedanken die mij in deze periode heeft geholpen, gesteund, gemotiveerd en geïnspireerd. Zonder hen zou ik dit resultaat nooit hebben bereikt. Een aantal mensen wil ik hieronder graag in het bijzonder bedanken.

Als eerste wil ik alle vrijwilligers en patiënten bedanken die zich bereid vonden om deel te nemen aan dit onderzoek. Zonder jullie bijdrage zou dit proefschrift niet tot zijn stand gekomen.

\section{Begeleidingscommissie}

Ik spreek mijn grote dank uit aan mijn promotor prof. dr. Jeroen Hendrikse. Jeroen, jij had het vertrouwen om mij te laten starten als promovendus. Destijds gewend aan het klinische werk bij de neurochirurgie, moest ik mij ineens het werk als onderzoeker meester maken. Met jouw enthousiasme en talloze ingevingen voorzag je mij van voldoende ideeën om er nog minstens vijf jaar onderzoek bij op te tellen. Toch wist jij mij altijd weer naar de kern van het onderzoek te bewegen, ook als ik weer eens afgedwaald was en allerlei zijsporen was ingeslagen. Je gaf mij alle vrijheid en mogelijkheden om mijzelf te ontwikkelen als arts-onderzoeker. Ook vond je altijd wel weer een moment in je overvolle agenda om even bij te praten en de lijn van het onderzoek weer helder voor ogen te krijgen. Ik vind het bijzonder hoe je dit allemaal doet en combineert. En naast nuttig, was het ook heel gezellig om onder het genot van een pizza, samen met jou en je zoontje Olivier, de laatste stand van zaken over het onderzoek door te nemen. Jeroen, bedankt!

Ook speciale dank aan mijn (co)promotoren dr. Irene van der Schaaf en dr. Bart van der Worp. Jullie gezamenlijke kennis en expertise waren de juiste mix om de radiologische en klinische kant van het onderzoek met elkaar te verbinden. Irene, jij wist mij elke keer weer scherp te houden op het onderwerp en einddoel van dit onderzoek. Ik hoop in de toekomst nog veel van je te leren op de werkvloer. En nu mag ik je ook van harte feliciteren met het verkrijgen van de titel Associate Professor, een kroon op al je werk! Bart, door je scherpe analyse tilde je het onderzoek naar een hoger niveau en wist je de klinische relevantie te bewaken. In de laatste fase van mijn onderzoek werd jij professor. Waar je eerst mijn copromotor was, ben je hierdoor mijn promotor geworden. Van harte gefeliciteerd met deze prachtige prestatie! Beste Irene en Bart, dank jullie wel voor jullie begeleiding de afgelopen jaren! 


\section{Beoordelingscommissie}

Graag wil ik ook de leden van de beoordelingscommissie bedanken voor de tijd en moeite die zij gestoken hebben in het kritisch lezen van het proefschrift en het opponeren tijdens mijn verdediging: prof. dr. Lenny Verkooijen, prof. dr. Birgitta Velthuis, prof. dr. Pim de Jong, prof. dr. Gabriël Rinkel en prof. dr. dr. Aad van der Lugt.

\section{‘Mentor' / Copromotor}

Dr. Anja van der Kolk, Anja, aan jou kan ik wel een heel hoofdstuk wijden. Jouw hulp was onmisbaar! Met onvermoeibare inzet stond je altijd klaar om met mij mee te denken, wist je mij altijd weer te motiveren en hielp je mij op weg als het schrijven niet wilde vlotten. Maar niet alleen op onderzoeksniveau wil ik je bedanken; ook op momenten dat ik 'onderzoeks-moe' was of mijn overvolle planningen volledig in de soep liepen, hielp je mij als een ware mentor. Met jouw strakke planning skills bracht je weer structuur aan in mijn chaos en wist ik de rode draad van zowel het onderzoek als het 'echte leven' vast te houden. Ik herinner mij nog de diverse momenten waarbij we alle deadlines, to-do-lists en prioriteitenlijstjes bij elkaar verzamelden en we er één gelikte planning van maakten. Gelukkig kon dat wel onder het genot van een goede lunch, bijvoorbeeld bij 'The Streetfood club' met een afsluitende cocktail, jouw guilty pleasure. Volgens mij moeten we die maar eens drinken zonder alle planningen, ter afronding van dit traject. Anja, ik kan je niet genoeg bedanken, jouw hulp tijdens dit traject was bijzonder waardevol. Update: In de laatste fase van mijn onderzoek werd jij alsnog officieel gevraagd om de rol als copromotor te vervullen. Fantastisch nieuws en meer dan verdiend!

\section{Paranimfen}

Dank aan mijn paranimfen dr. Erwin Krikken en dr. Lisa van der Kleij. Jullie waren mij al voorgegaan en dankzij jullie kon ik met een gerust hart naar mijn promotie uitkijken. Dank voor jullie organisatie, steun, tips en adviezen in aanloop naar mijn verdediging.

\section{Vaatwandteam}

Ook dank aan het Vaatwandteam; naast dr. Anja van der Kolk ook dr. Jaco Zwanenburg, dr. Jeroen Siero, dr. Anita Harteveld en dr. Nikki Dieleman. Jaco, dank voor alle gesprekken, maar ook voor de uitleg de je mij hebt gegeven over de technische aspecten van de MRI. Ik kon altijd bij je aankloppen als ik weer eens iets niet begreep. En Jeroen, altijd in voor een biertje, maar ook een echte expert op het gebied van de MRI. Ook bij jou kon ik voor al mijn vragen terecht en dat deed 
ik dan ook regelmatig. Jullie zorgden ervoor dat de vaatwandsequenties werden geoptimaliseerd en dat ook de technische kant van het onderzoek klopte. In mijn huidige werk komt jullie overgedragen kennis nog regelmatig van pas! Anita, mijn dagelijkse collega op vaatwandgebied, ik wat vaker 'voor in het hoofd' (IVI) en jij wat vaker 'buiten' (ex-vivo) of 'achter in het hoofd' (PIVI). Jij hebt mij wegwijs gemaakt op de afdeling, in het scannen en in alle lopende projecten. We hebben heel wat uurtjes scannend doorgebracht, maar ook presentaties geoefend en over ideeën gespard. Ik weet niet hoe vaak ik wel niet bij jou heb aangeklopt om weer 'even iets te bespreken'. Dank voor al je hulp en tips! Nikki, jij was mijn andere 'vaatwandcollega' en expert op het IVI-project toen ik begon. Jij hebt mij de fijne kneepjes van het project bijgebracht en mij hierop ingewerkt. Dank voor al je uitleg, adviezen en 'inside' projectinformatie, waardoor ik het project uiteindelijk van je over kon nemen. En tenslotte, Carlo Lucci, aan wie ik het stokje heb mogen overdragen. Carlo, voor jou hoef ik het niet meer in het Italiaans te vertalen, want jij hebt in zo'n rap tempo vloeiend Nederlands geleerd; mijn complimenten! Succes met de laatste loodjes van het onderzoek! Tof dat we collega's blijven nu je ook in opleiding bent tot radioloog.

Niet direct bij het Vaatwandteam betrokken, maar wel nauw hieraan gerelateerd; dank aan Maarten Zwartbol en Rashid Ghaznawi. We hebben heel wat discussies gevoerd over ons onderzoek en de resultaten en op epidemiologische wijze de risicofactoren becommentarieerd. Gelukkig ging het niet alleen hierover, maar ook regelmatig over de politiek, het dagelijkse leven en Bitcoins. Een welkome afwisseling.

\section{Collega's en betrokkenen}

Voor het scannen van klinische patiënten was er altijd hulp van de MRIlaboranten, waaronder Guus, Niels, Gerrit, Daniel en Gideon. Dank voor al jullie ondersteuning tijden het scannen. Ook dank aan dr. Hans Hoogduin en Fredy Visser. Als er weer eens een foutmelding was of ik het systeem niet aan de praat kreeg, kon ik altijd een beroep doen op jullie waanzinnige technische kennis over de 7T MRI.

Een artikel schrijven doe je nooit alleen. Daarom wil ik ook alle medeauteurs bedanken voor hun hulp en de input die zij hebben geleverd voor het schrijven van de artikelen. Naast de al eerdergenoemde collega's/coauteurs wil ik ook dr. Laurens de Cocker, dr. Jeroen de Bresser, prof. dr. Michiel Bots en drs. Theo Witkamp bedanken voor alle hulp en inspanning die jullie hebben geleverd aan de artikelen die in dit proefschrift zijn opgenomen. Uiteraard wil ik ook de andere collega's bedanken waarmee ik direct of indirect aan de overige artikelen heb samengewerkt. 
Ook dank aan Roy Sanders en Chris van Kesteren, van de multimedia-afdeling. Jullie hebben mij ontzettend geholpen met het maken van alle figuren die nodig waren voor de artikelen in dit proefschrift. En niet alleen met het maken, maar ook met het telkens opnieuw aanpassen van de figuren na iedere revisieronde om de reviewers tevreden te stellen. Ik stond iedere keer weer met verbazing te kijken hoe vliegensvlug jullie dat in Photoshop klaarspelen. Dank daarvoor.

Daarnaast wil ik alle personen bedanken die mij op welke wijze dan ook hebben geholpen of ondersteund tijdens dit PhD-traject, waaronder de secretaresses Sylvia Alberto en Marja Kool voor al jullie ondersteuning en Shanta Kalaykhan van het trialbureau voor de juridische en praktische ondersteuning bij alle projecten.

Dank ook aan al mijn collega's in het Meander Medisch Centrum in Amersfoort voor de leerzame eerste twee jaar van mijn specialisatie. Met name aan mijn opleider dr. Jasper Florie; bedankt dat je mij de tijd en mogelijkheden gaf om de (lange) eindsprint te maken om dit onderzoek af te kunnen ronden en $\mathbf{d r}$. Cornelia Schaefer-Prokop, voor je bemoedigende gesprekken en wijze adviezen om het leven naast het onderzoek niet te vergeten.

Verder wil ik mijn collega's in het UMC Utrecht bedanken voor de ruimte die ik heb gekregen om alles af te ronden. Vooral mijn opleiders dr. Rutger Jan Nievelstein en drs. Monique Hobbelink, die mij met een (stevig) duwtje in de rug over de laatste hobbels van mijn onderzoek heen hebben geholpen. Cliché, maar waar; de laatste loodjes wegen het zwaarst. Dank voor jullie hulp en krachtige optreden om de puntjes op de i te zetten en mijzelf en anderen daarin niet te vergeten.

\section{T-onderzoekers}

Uiteraard wil ik ook alle 7T-onderzoekers en de Q2-gang niet vergeten. Deze groep is te groot en te dynamisch om iedereen persoonlijk te kunnen bedanken, maar daarom een niet minder gemeende dankzegging. De afgelopen jaren heb ik heel wat collega's zien komen en gaan. Samen hebben we talloze borrels, koffiepraatjes, spontane discussies, PhD-feestjes, barbecues en andere mooie momenten beleefd. We waren altijd present op de feesten tijdens en na congressen, op de Christmasparty van het UMC, tijdens de UMC-estafettes, de fietsenrally en nog vele andere activiteiten. Regelmatig kwam een van jullie binnenvallen voor een welkome social talk (en vaak ook voor de snoeppot). Je kunt wel stellen dat deze groep samen mijn PhD-tijd heeft gemaakt! 


\section{Epi-Rad club}

Ook dank aan de Epi-Rad club waarbij we wekelijks wetenschappelijke discussies en praatjes hadden en waarin we op een veilige manier ons onderzoek en de eerste resultaten konden bespreken, voordat we ze aan de grote boze buitenwereld lieten zien.

Dank ook aan al mijn Epidemiologie collega's die ik tijdens mijn (postgraduate) master heb leren kennen, waaronder Willem Paul Gielis. We konden altijd mooi ouwehoeren over alles. Je statistische tips en trucs waren meer dan welkom.

\section{Cycling for Science}

Speciale dank ook aan de Cycling for Science (C4S) fietsgroep. Ik keek elke keer weer uit naar de maandagavond waarbij er op een hele andere manier onderzoek werd gedaan. In onze aero-designer outfits onderzochten we wie het snelste was in de tussensprints, het langst op kop reed en hoe we als groep het gemiddelde omhoog konden krijgen. Gelukkig werd er ook vaak ontspannen gekoerst. Een welkome afwisseling voor alle lange onderzoeksdagen; Alex, Casper, Fredy, Jannie, Peter, Remko, Cyril, Bart, Sander, Anita (en andere medesportievelingen), bedankt!

\section{Vrienden}

Dat zo'n PhD-traject ook voordelen kent, blijkt wel! Tijdens de afgelopen jaren zijn er vele mooie vriendschappen ontstaan.

Lisa van der Kleij, Erwin Krikken en Janot Takoya. Jullie heb ik al een paar keer, al dan niet impliciet, genoemd. Wat had ik zonder jullie gemoeten al die jaren! Onze vele etentjes, borrels, pubquiz-avonden, HIIT-tussenuurtjes, feestjes en weekendjes weg hebben de afgelopen jaren voor mij bijzonder en onvergetelijk gemaakt. ledere keer verschijnt er weer een hele grote glimlach op mijn gezicht als ik denk aan hoe we (met een aantal van ons), licht snakkend naar adem, op de top van de Mauna Kea (ruim 42000 m) in Hawaii stonden en aan het genieten waren van een magisch maanachtig landschap tijdens zonsondergang. Of hoe we tijdens een stormachtige nacht, naar ruim 20 meter diepte in de stille oceaan doken om reusachtige manta rays rakelings langs ons heen te zien zwemmen. Of 's nachts naar de sterren keken naast een actief spuwende vulkaan, adembenemend... Dat maakte overigens de weekendjes op Texel tussen de lammetjes of het ontspannen bij de open haard in Schagen niet minder mooi. Op naar nog veel meer mooie momenten en weekendjes weg! Lieve Lisa, wat een geweldige vriendin, fantastisch kamergenootje en (tot voor kort) buurvrouw ben jij. Je kende mij uiteindelijk zo goed dat je bij binnenkomst al aan mijn gezicht kon zien hoe het met mij ging. Ideaal, als ik weer eens aan het 
worstelen was en afleiding of tips nodig had. Dank voor al je wijze raad en luisterend oor de afgelopen jaren! Erwin, altijd in voor een praatje, goed gehumeurd en vol energie aanwezig op de afdeling. Regelmatig was jij de kartrekker als er weer eens iets geregeld of georganiseerd moest worden. Ik denk dat jij ervoor gezorgd hebt dat er een (h)echt teamgevoel op de afdeling was. We hebben heel wat activiteiten ondernomen de afgelopen jaren en ik merk dat onze karakters in veel opzichten overeenkomsten vertonen, wellicht door onze oostelijke roots. Dank voor alles en op naar nog heel veel mooie momenten! Janot, ook met jou heb ik heel wat tijd doorgebracht de afgelopen jaren. Was het niet tijdens het proeven van je eigen gebrouwen bier of een van je fantastische Oosterse gerechten, dan was het wel in de sportschool. Wat een sportbeest ben je; fluitend til jij het ene na het andere gewicht (of tegelijk) de lucht in. Onze vriendschap had ik zeker niet willen missen. Ik kan niet wachten tot de volgende batch geproefd mag worden! Alex Bhogal, thanks bro, voor al je fietsritjes, goede gesprekken en afleidende foto's. Hoe hard ik het ook probeer, zowel op de fiets als in de kroeg houd ik je niet bij. Ik heb genoten van onze talloze analyses, diepgaande discussies, maar ook volledig zinloze gesprekken in en buiten de kroeg. Ik ben de tel kwijtgeraakt van de hoeveelheid avonden die wij zo hebben doorgebracht. Wat een fantastische tijd, zodra het weer kan, moeten we dat maar weer gaan herhalen! Ik hoop dat we snel weer samen weer kunnen fietsen en afsluiten met een biertje op het terras. Ik ben wel weer eens toe aan een chill-out (of was het nu een chill-down?) momentje. Tijl van der Velden, dank voor alle ontspannende borrels en barbecues! Het lijkt soms wel alsof jouw hele leven een grote borrel is, hoe doe je dat toch? Die uitleg zie ik graag tegemoet, wellicht op een volgende BBQ. Maar dan wel een beetje zoutarm graag; want weet je hoeveel zout overal in zit? Volgens mij hadden we deze discussie nog steeds niet beëindigd. En natuurlijk ook Catalina Arteaga, dank voor je gastvrijheid en goede gezelschap op alle barbecues en feestjes!

Stefano Mandija en Deji Adams, dank voor onze fantastische vakantie(s) na de congressen in Singapore en Hawaii. Ultiem ontspannen na een week vol kennis en wetenschap. Maar Stefano, neem volgende keer alsjeblieft een eigen rugzak mee voor je hele apotheek aan medicijnen.

Tim Veersema, wij hebben vrijwel een identiek carrièrepad afgelegd waarbij we tevens aardig wat dezelfde hobbels zijn gepasseerd en dat zorgde vaak voor herkenbaarheid. Maar naast de hobbels kunnen we gelukkig ook veel mooie momenten delen, zoals de vakantie in Maleisië of de assistentenborrels. Nu we beide in opleiding zijn tot interventieradioloog, bewandelen we weer dezelfde weg. Dat onze paden nog maar vaak zullen kruisen! 
Jan Jaap Janssen en Maarten Jalink, van Groningse studentenfeestjes tot de Surinaamse jungle. We hebben al aardig wat meegemaakt en ik ben blij dat onze vriendschap nog steeds voortduurt. Uit de immer veranderende naam van onze WhatsApp-groep met Jasper blijkt wel hoe dynamisch we zijn, maar dat overeenkomsten tegelijkertijd blijven overheersen. Ik denk met ontzettend veel plezier terug aan onze avontuurlijke reizen. Jan Jaap, met jou op Curaçao, maar ook aan onze gezamenlijke waanzinnige 'Jungle Expeditie' diep in de Surinaamse Jungle; wie gelooft er nu dat we in het Amazonegebied ons eigen voedsel moesten verzorgen? Met blote handen krokodillen hebben gevangen, piranha's (en meer...) hebben gegeten en hebben geslapen in een hangmatje omringd door vogelspinnen en los rondlopende poema's? Toch even schrikken toen we 's ochtends de grote klauwen in de grond zagen dichtbij ons kamp. Ik kijk uit naar een mooie reüniereis! Ik zal eens googlen of er SUV's zijn met speciale kinderzitjes voor Frederique en Noor. Het is in ieder geval een mooi verhaal voor hen voor het slapen gaan, verteld door jullie als kersverse pappa's.

Dank ook aan 'De Toppertjes'; mijn vrienden Jan Lindenholz, Lucel Mulder en André Verhoek, die ik al ken sinds de basisschool (en Jan natuurlijk nog langer). Met jullie ben ik opgegroeid in Rouveen en samen hebben we een hoop meegemaakt. Jullie kennen mij door en door en ik weet dat jullie altijd voor me klaarstaan. Dank jullie wel voor jullie steun door alle jaren heen! Als jonkies begonnen we de wereld te verkennen vanuit ons vaste honk en inmiddels zijn we uitgevlogen over heel Nederland en hebben we al heel wat van de wereld gezien. Gelukkig hebben we onze mannen-weekendjes nog waarbij we uit alle hoeken van het land weer samenkomen, ik kijk er nu alweer naar uit!

René van den Berg, hoewel je nu in Zwitserland woont, spreken we elkaar nog regelmatig. Ik mis je man! Hoe relaxt was het dat we voorheen gewoon onverwachts bij elkaar binnen konden vallen en met een biertje op de bank de hele avond konden chillen. Natuurlijk weet ik dat dit, met een wat langere reisafstand, nog steeds kan. Als ik alle fantastische foto's zie van jou met Annelot in Zwitserland, is het wel duidelijk dat jullie het gelukkig goed naar je zin hebben daar. Tijd om maar eens een bezoekje in te plannen!

Naast al het onderzoek was er ook tijd voor een mooi avontuur. Tijdens mijn onderzoeksperiode heb ik samen met The Big 6; Eva Mulder, Linda Rolf, Kyra Kingma, Bart Swolfs en Ruth Gussenhoven de Africa Classic gefietst; een offroad mountainbiketocht door Kenia en Tanzania voor het goede doel; Amref Flying Doctors. Een jaar lang hebben wij ons ingezet en voorbereid om dit mogelijk te maken. Wat een bijzonder avontuur hebben we mogen beleven. Over de zinderende dorre savanne's, 's avonds dansen met de Masai, wakker worden met het zicht op de Mount Kilimanjaro en giraffes die langs onze tentjes lopen. 
Het stof is nog steeds niet uit mijn fietskleren en van mijn mountainbike. Eva, bedankt voor je enthousiasme en voor alle hulp in de eerste periode van mijn onderzoekstraject. Linda, dank voor al je hulp en bemoedigende woorden. Kyra en Bart, een powerkoppel dat altijd voor anderen klaarstaat, dank jullie wel! Ruth, de vrolijke optimist en de sfeermaker in de groep, bedankt!

En niet te vergeten Robbert Bloksma; dank je wel voor alle mooie en gezellige avondjes. Ons laatste biertje was in het weekend voordat alles dichtging vanwege de lockdown, een memorabel moment. Nu de terrassen langzaamaan weer opengaan, wordt het volgens mij weer tijd voor een biertje!

Noor, bedankt voor je luisterend oor, oprechte interesse en heerlijke kookkunsten. Jouw kook-skills zijn zo goed dat het eten zelfs nog voortreffelijk smaakt wanneer ik weer eens veel te laat aan kom zetten. Na dit traject heb ik daar geen excuus meer voor. Ik kijk uit naar nog vele gezellige en culinaire avonden met jou en Wouter, maar eerst genieten tijdens een relaxte (of toch actieve) vakantie in Frankrijk met ons vieren (+ Suus)!

Dank ook aan Kaatje, Anne-Marie, Ilse en Famke voor alle support die jullie mij gaven in dit hele onderzoekstraject, ook wanneer ik weer eens lekker aan het klagen was over alle strubbelingen.

\section{Familie}

Tenslotte wil ik mijn familie bedanken. Ik kan mijzelf gelukkig prijzen met mijn grote familie. Ondanks dat het onderzoek en werk er vaak voor zorgden dat ik jullie de afgelopen jaren minder heb gezien dan ik eigenlijk had gewild, wist ik dat jullie mij onvoorwaardelijk steunen en kan ik mij geen leven zonder jullie voorstellen.

Mijn broers Jarno en Erik en mijn broertje Alwin. Bedankt voor jullie steun en sportiviteit. De fietstocht naar de Mont Ventoux en de vele ski/snowboard-uitjes zal ik, ondanks (of dankzij) de hersenschudding, kapotte knie en gebroken ribben, niet snel vergeten. Het was heerlijk om op deze momenten met jullie van de natuur te genieten, tot het uiterste te gaan en even niet met mijn werk en onderzoek bezig te zijn. Fijn en waardevol om dit fanatisme en enthousiasme met jullie te kunnen delen! En Jarno en Erik, vol bewondering zie ik als trotse oom hoe jullie je rol als vader vervullen. Jullie weten de naam Lindenholz goed in stand te houden! En Alwin, met jouw doorzettingsvermogen op sportief vlak, ga jij ook zeker weten een mooie carrière tegemoet! 
Mijn zus Gerjanne en zusje Jetty. Ik weet dat jullie altijd met mij mee leven. Prachtig om jullie zo trots te zien genieten van Floris en Boaz. Het geeft een goed gevoel om zo'n grote familie om me heen te hebben.

Als inmiddels onmisbaar gedeelte van het gezin, natuurlijk ook dank aan mijn schoonzussen Hanneke en Anouk en zwagers Henri en Jan Mark.

Papa en mama, jullie nuchtere opvoeding heeft er mede voor gezorgd dat ik nu ben wie ik ben. Jullie leerden mij hard te werken en ook mijn doorzettingsvermogen heb ik niet van een vreemde. Ik weet dat jullie altijd achter mij staan en mij het beste gunnen in al mijn duizenden overwegingen. Het is elke keer weer een warm welkom om bij jullie (thuis) langs te komen en om daarna weer volgestopt met lekkernijen in de trein naar Utrecht af te reizen. $\mathrm{Na}$ al mijn reizen besef ik mij steeds meer hoe warm en vertrouwd mijn thuis is.

Mijn schoonouders Joop en Els, dank jullie wel voor jullie hartelijkheid en welgemeende interesse in mij, mijn onderzoek en werk. Dank ook voor al jullie fijne etentjes en vakanties waarbij ik weer even 'op kon laden'. Heel mooi om te zien hoe trots jullie zijn op Anouk.

Allerliefste Anouk, hoeveel dank ben ik jou wel niet verschuldigd. Jij bent vaak mijn stille en drijvende kracht om toch weer door te gaan als ik het even niet meer zie zitten. Ik ben ontzettend blij dat ik jou heb leren kennen en geniet daar elke dag van. Regelmatig bedenk je in al je creativiteit weer iets om samen te ondernemen en maak je zo het leven steeds weer nóg een stukje mooier. Ik ben je ongelooflijk dankbaar voor je eindeloze geduld, liefde, zorgzaamheid en steun die je mij ieder dag onvoorwaardelijk geeft. Maar nu is dan eindelijk het onderzoek af, en wordt het tijd voor elkaar! Ik kan niet wachten om met jou de wereld te ontdekken! 


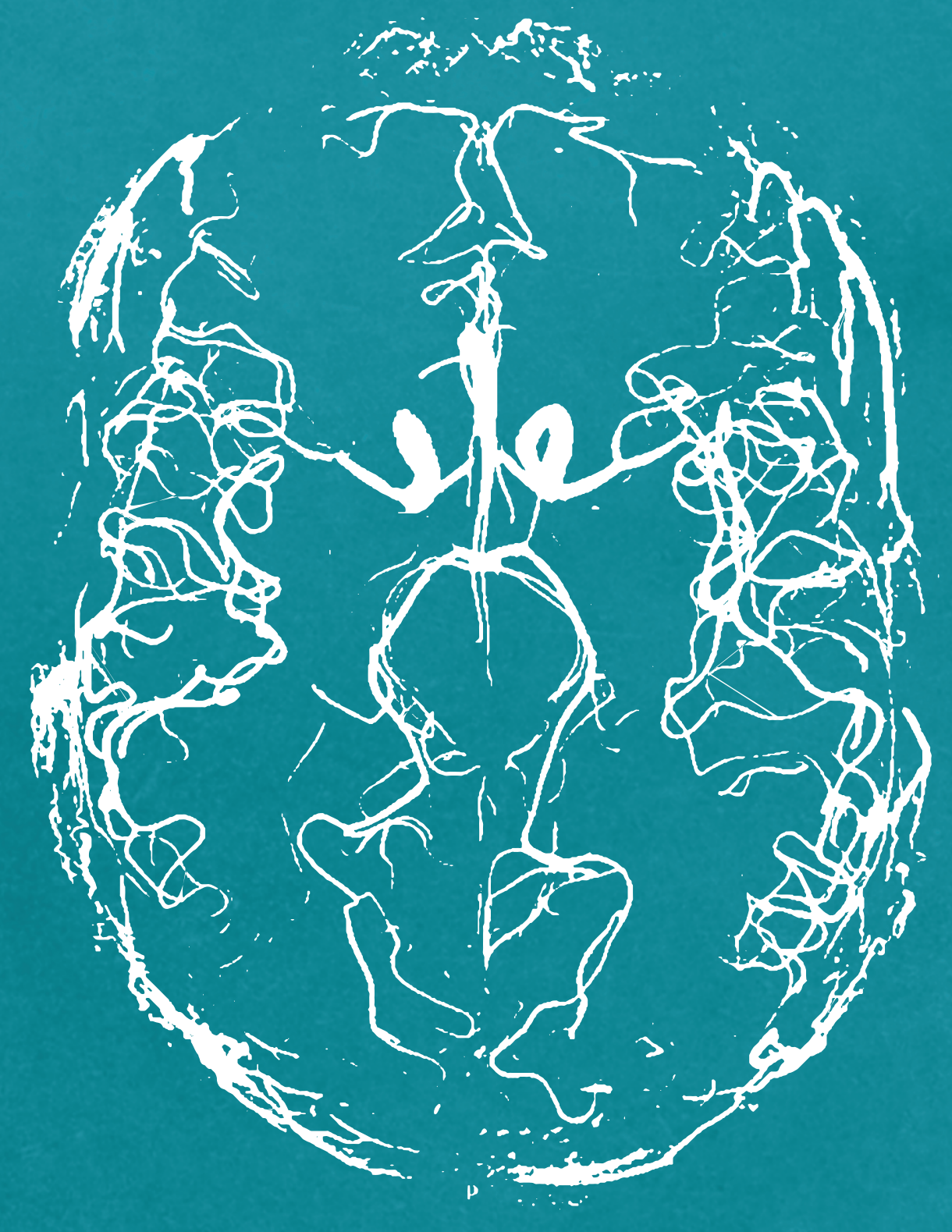


ADDENDUM IV

Curriculum vitae 
Arjen Lindenholz werd geboren op 18 december 1986 in Rouveen, Overijssel. Na het behalen van zijn vwo-diploma aan de Pieter Zandt Scholengemeenschap te Kampen stapte hij na een jaar Verpleegkunde in 2006 over op de studie Geneeskunde aan de rijksuniversiteit Groningen. In 2009 begon hij aan de Master Geneeskunde, waarvan hij ruim een jaar in het Sint Elisabeth Hospitaal op Curaçao heeft gewerkt. Het laatste jaar van zijn Master sloot hij af aan het Krembil Neuroscience Centre in het Toronto Western Hospital in Canada. Met een beurs van de Hartstichting deed hij hier, als onderdeel van het talentenprogramma GIPS-M, onderzoek naar spinale durale arterioveneuze fistels. In 2012 ontving hij zijn masterdiploma Geneeskunde met de distinctie 'Cum Laude'.

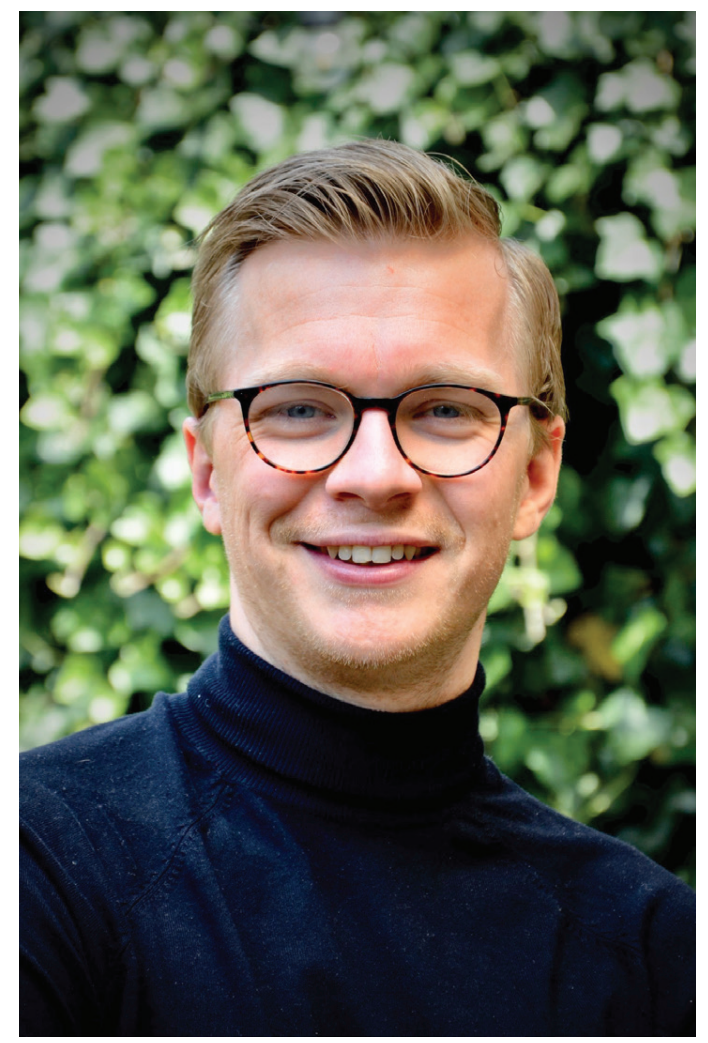

Vervolgens werkte hij twee jaar als arts-assistent op de afdeling neurochirurgie in het UMC Groningen en het UMC Utrecht.

In 2015 startte zijn promotietraject bij de afdeling Radiologie in het UMC Utrecht met als onderwerp 'Intracraniële Vaatwand MR Imaging'. Tijdens dit promotietraject behaalde hij in 2018 de Postgraduate Master Epidemiology met als specialisatie Clinical Epidemiology (GPA 4.0).

Vanaf juni 2018 is hij in opleiding tot specialist op de afdeling Radiologie (AIOS) in het Meander Medisch Centrum Amersfoort en het UMC Utrecht. 
Arjen Lindenholz was born on December $18^{\text {th }} 1986$ in Rouveen, Overijssel. After finishing high school in 2005 at Pieter Zandt Scholengemeenschap in Kampen, he studied Nursing for a year, after which he switched to Medicine at the University of Groningen. In 2009 he began a Master program in Medicine, during which he worked for over a year at the Saint Elisabeth Hospital in Curaçao. After joining the talent program GIPS-M, he continued to finish his Master degree at the Krembil Neuroscience Centre in Toronto, Canada. Funded by a grant from the Dutch Heart Foundation he did research at the Toronto Western Hospital on spinal dural arteriovenous fistulas. In 2012 he graduated from his Master program with the distinction 'Cum Laude'.

Thereafter, he worked for two years as a neurosurgical resident at the Neurosurgical department at UMC Groningen and the UMC Utrecht.

In 2015 he started with his PhD research program at the Radiology department at UMC Utrecht researching the topic of 'Intracranial Vessel Wall MR Imaging'. During this PhD program he graduated the Postgraduate Master program in Epidemiology with as specialization Clinical Epidemiology (GPA 4.0).

Since June 2018, Arjen is a Radiology resident to become a specialist (AIOS) at the Radiology department at Meander Medisch Centrum Amersfoort and UMC Utrecht. 